Aus der Poliklinik für Kieferorthopädie (Prof. Dr. med. dent. D. Kubein-Meesenburg) im Zentrum Zahn-, Mund- und Kieferheilkunde der Medizinischen Fakultät der Universität Göttingen

\title{
Einfluss zweier Bandscheibenprothesen auf die Kinematik des C3/C4-Segmentes
}

\author{
INAUGURAL - DISSERTATION
}

zur Erlangung der Doktorgrades der Medizinischen Fakultät der Georg-August-Universität zu Göttingen

\author{
vorgelegt von \\ Markus Wagner \\ aus \\ Lutherstadt Wittenberg
}

Göttingen 2013 
Dekan:

Prof. Dr. rer. nat. H. K. Kroemer

I. Berichterstatter/in:

II. Berichterstatter/in:

III. Berichterstatter/in:

Tag der mündlichen Prüfung: $\quad$ 17.09.2014 


\section{Inhaltsverzeichnis}

Seite

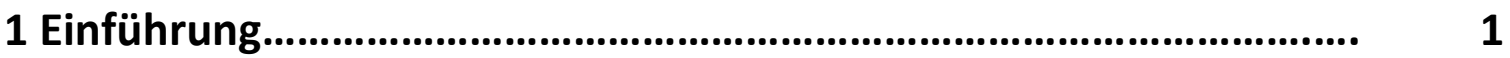

1.1 Einleitung

1.2 Anatomische Grundlagen .............................................................................

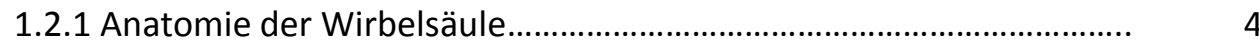

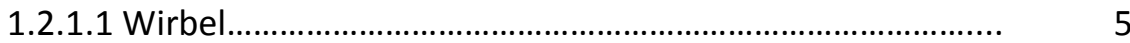

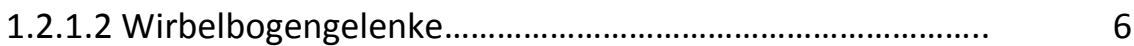

1.2.1.3 Zwischenwirbelscheiben.................................................... 7

1.2.1.4 Bandapparatur der Wirbelsäule............................................... 8

1.2.1.5 Muskulatur der Wirbelsäule................................................... 9

1.3 Pathologische Veränderungen der Halswirbelsäule............................................... 10

1.3.1 Degenerative Erkrankungen der Halswirbelsäule................................. 11

1.3.2 Zervikaler Bandscheibenvorfall.......................................................... 12

1.4 Zervikale Bandscheibenprothesen...................................................................... 14

1.4.1 Historische Entwicklung.......................................................................... 14

1.4.2 Einteilung der Bandscheibenprothesen ................................................. 16

1.4.3 Indikationen und Kontraindikationen.................................................... 17

1.4.4 Anforderungen an eine Bandscheibenprothese................................... 18

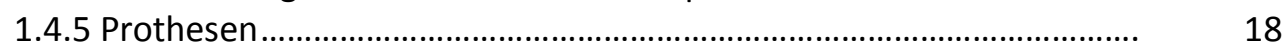

1.4.5.1 Prestige ${ }^{\circledR}$ LP Cervical Disc System.......................................... 18

1.4.5.2 Bryan ${ }^{\circledR}$ Cervical Disc System............................................... 19

1.5 Biomechanik................................................................................................ 20

1.5.1 Bestimmung der Bewegungsstruktur................................................. 20

1.5.2 Grundlagen der Biomechanik............................................................ 21

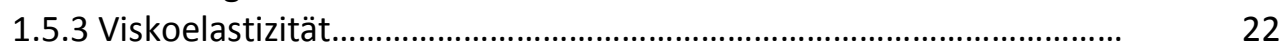

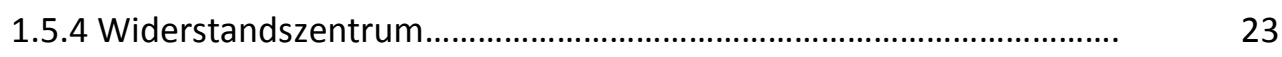

1.5.5 Biomechanische Eigenschaften intakter Segmente............................ 24

1.6 Fragestellung .................................................................................................. 25

2 Material und Methoden................................................................... 26

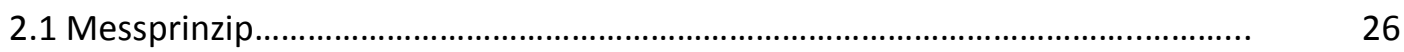

2.2 Material und Morphologie................................................................................. 26

2.2.1 Halswirbelpräparate........................................................................ 26

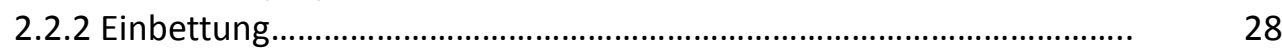

2.2.3 Prothesenimplantation..................................................................... 29

2.3 Messsystem ............................................................................................. 32

2.3.1 Messapparatur .................................................................................

2.3.2 Applikation von Kräften................................................................... 33

2.3.2.1 Axiale Vorlast........................................................................

2.3.2.2 Drehmoment.....................................................................

2.3.3 Taster, Messinterface und Software..................................................... 36

2.4 Positionsänderung im Raum - Konzept der Schraubachse..................................

2.5 Bandscheibenmodell........................................................................................

2.6 Modellmessung Prestige ${ }^{\circledR}$ LP Cervical Disc System............................................. $\quad 39$

2.7 Bestimmung des Widerstandszentrums................................................................ 39

2.8 Graphische Präsentation der Messdaten............................................................. 4

2.9 Statistische Datenanalyse ...............................................................................

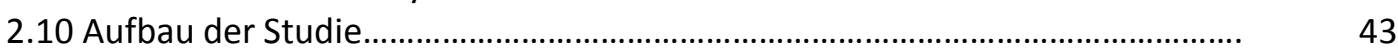


3.1 Probemessung - Präzisionsschraube................................................................ 47

3.2 Überprüfung des Pumpsystems....................................................................... 48

3.3 Probemessung - Bandscheibenmodell............................................................. 48

3.4 Modellmessung - Prestige ${ }^{\circledR}$ LP Cervical Disc System ......................................... 50

3.5 Bestimmung des Widerstandszentrums........................................................... 52

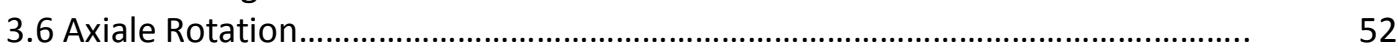

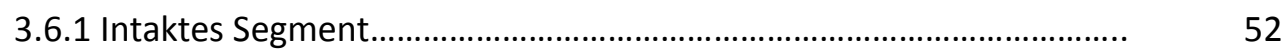

3.6.1.1 Lage und Wanderung der Rotationsachse....................... 53

3.6.1.2 Ausrichtung der Rotationsachsachse............................... $\quad 57$

3.6.1.3 Drehwinkel-Drehmoment-Kennlinie,

Bewegungsausmaß und Steifigkeit................................... 58

3.6.2 Bandscheibenprothesen Prestige ${ }^{\circledR}$ LP Cervical Disc System \& Bryan $^{\circledR}$ Cervical Disc System............................................................... 60

3.6.2.1 Lage und Wanderung der Rotationsachse........................ 60

3.6.2.2 Ausrichtung der Rotationsachse nach

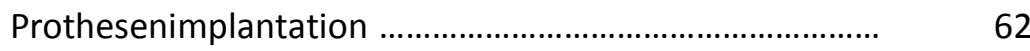

3.6.2.3 Schwerpunkt.................................................................... 65

3.6.2.4 Initiale Migrationsrate (IMR) der Rotationsachse............. 66

3.6.2.5 Drehwinkel-Drehmoment-Kennlinie,

Bewegungsausmaß und Steifigkeit................................. 67

3.7 Lateralflexion

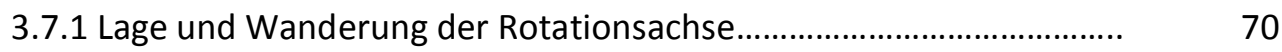

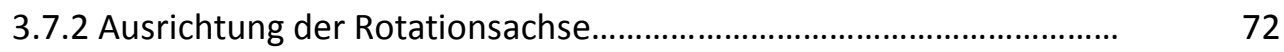

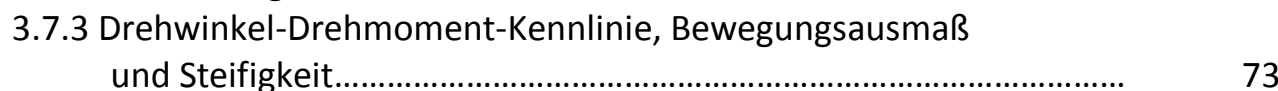

3.8 Ventralflexion-Extension.................................................................................. 76

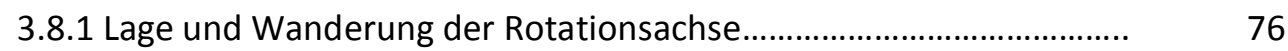

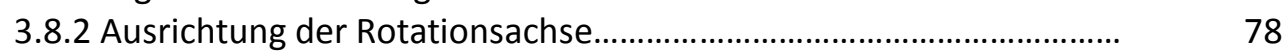

3.8.3 Drehwinkel-Drehmoment-Kennlinie, Bewegungsausmaß

und Steifigkeit................................................................................ $\quad 79$

3.9 Qualitative Beurteilung der Gelenkflächen ................................................... 81

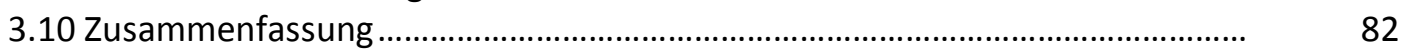

4 Diskussion....................................................................................... 85

4.1 Bewegungsstruktur des Halswirbelsäulensegmentes C3/C4 .......................... 85

4.1.1 Axiale Rotation.............................................................................. 86

4.1.2 Lateralflexion........................................................................... 96

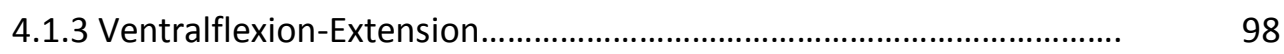

4.1.4 Biomechanische Schlussfolgerung ................................................. 100

4.2 Methodendiskussion................................................................................... 101

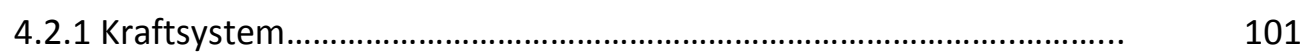

4.2.1.1 Kompressive Kraftkomponente..................................... 101

4.2.1.2 Drehmoment................................................................... 102

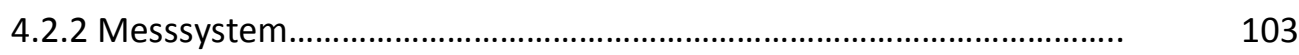

4.2.3 Präparate............................................................................................. 103

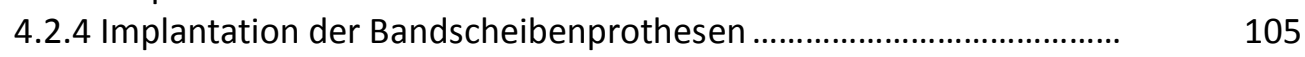

4.3 Probemessungen.................................................................................... 106

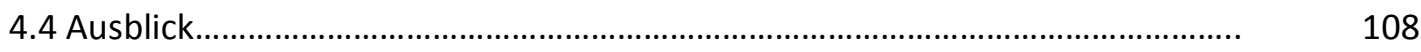


Seite

5 Zusammenfassung.............................................................................. 110

5.1 Stand der Wissenschaft................................................................................. 110

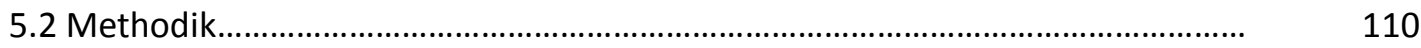

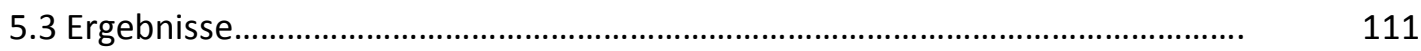

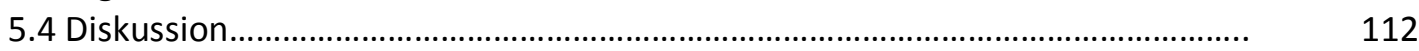

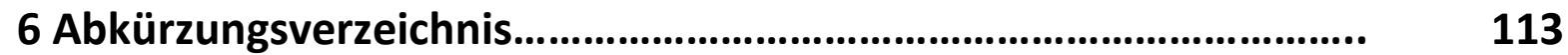

7 Literaturverzeichnis.............................................................................. 116 


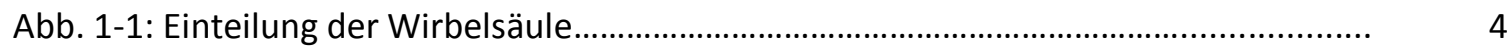

Abb. 1-2: Anatomie des Halswirbels.................................................................................. 5

Abb. 1-3: Frontale Ansicht eines HWS-Segmentes \& vergrößerte Darstellung des Bereiches der Uncovertebralgelenke............................................................. 6

Abb. 1-4: Seitliche Darstellung eines HWS- Segmentes \& vergrößerte Darstellung eines Facettengelenks.

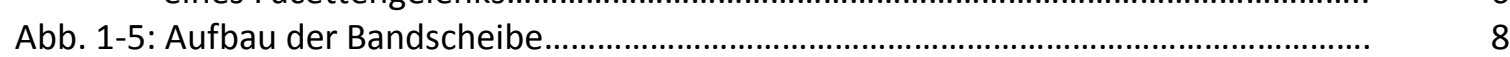

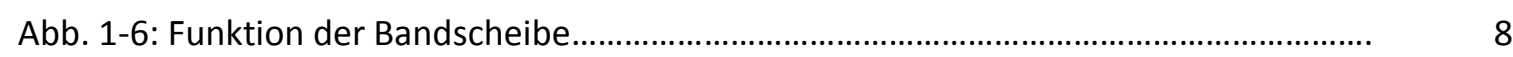

Abb. 1-7: Bandapparatur der Halswirbelsäule auf Höhe des 4. und 5. Halswirbels............... 8

Abb. 1-8: Einteilung und Funktion der ventralen Halsmuskulatur............................................. 9

Abb. 1-9: Einteilung und Funktion der autochthonen Rückenmuskulatur............................ 10

Abb. 1-10: Schematische Darstellung der Wirbelsäulendegenerationsarten......................... 11

Abb. 1-11: Schweregradeinteilung der Bandscheibendegeneration...................................... 12

Abb. 1-12: MRT-Aufnahme eines Bandscheibenvorfalls zwischen dem

4. und 5. Halswirbel

Abb. 1-13: Seitliche Röntgenaufnahme der HWS nach Implantation eines

Kugelimplantates zwischen C5/C6.................................................................. 14

Abb. 1-14: Bandscheibenprothesen der Firma Medtronic Sofamor Danek............................ 15

Abb. 1-15: Schematische Darstellung der Prothesen-Einteilung anhand des Designs......... 16

Abb. 1-16: Prestige ${ }^{\circledR}$ LP Cervical Disc System......................................................................... 19

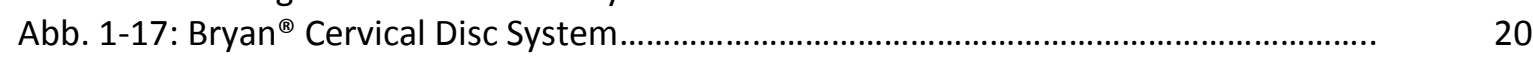

Abb. 1-18: Bryan ${ }^{\circledR}$ Cervical Disc System: Querschnitt................................................................ 20

Abb. 1-19: Bewegung eines Körpers im dreidimensionalen Raum....................................... 21

Abb. 1-20: Bestimmung der Richtung eines Drehmomentes................................................. 22

Abb. 1-21: Schema einer Synarthrose................................................................................. 23

Abb. 1-22: Auswirkung einer $\operatorname{Kraft} F\left(F_{x}, 0, F_{z}\right)$ auf eine Synarthrose..................................... 23

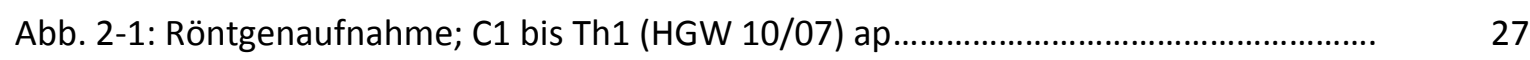

Abb. 2-2: Röntgenaufnahme; C1 bis Th1 (HGW 10/07) seitlich............................................. 27

Abb. 2-3: Röntgenaufnahme; eingebettetes Präparat (HGW 16/07) ap................................ 29

Abb. 2-4: Röntgenaufnahme; eingebettetes Präparat (HGW 16/07) seitlich.......................... 29

Abb. 2-5: HGW 16/07 - Extraktion der Bandscheibe............................................................... 30

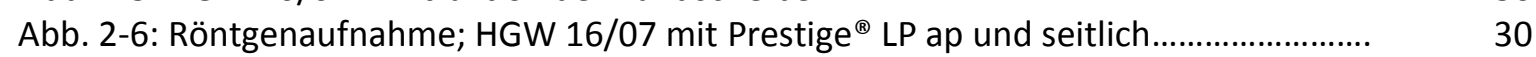

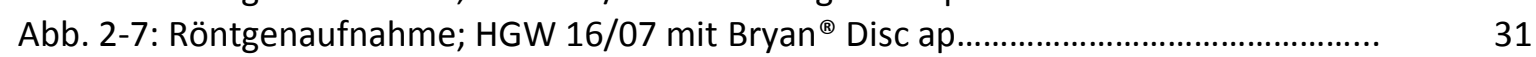

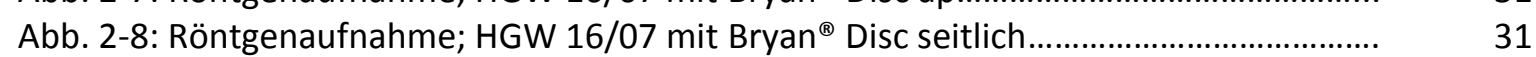

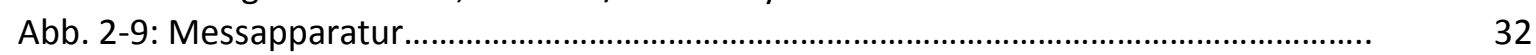

Abb. 2-10: Positionen der axialen Vorlast..............................................................................

Abb. 2-11: Schematische Darstellung des Messsystems.......................................................

Abb. 2-12: Pumpsystem mit Wasserbehältern .......................................................................

Abb. 2-13: Schema des Leitungssystems eines Wasserbehälters .......................................... $\quad 35$

Abb. 2-14: Schema des Versuchsaufbaus zur Applikation eines Drehmomentes

Abb. 2-15: Verwendeter Längenmesstaster der Firma Mahr GmbH Göttingen

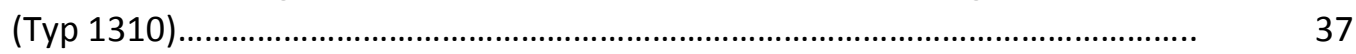

Abb. 2-16: Anordnung der Taster in der Messapparatur ......................................................

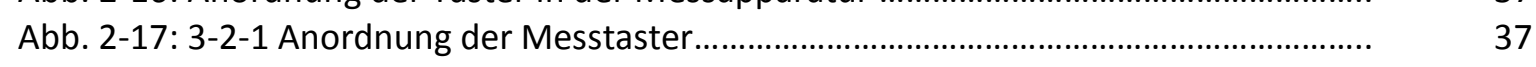

Abb. 2-18: Gummipuffer mit Facettengelenkimitation......................................................... 38

Abb. 2-19: Tasterwerte T1 bis T6 bei Belastung außerhalb des Widerstandszentrums...... $\quad 40$ 
Abb. 2-20: Tasterwerte T1 bis T6 bei Belastung im Widerstandszentrum ...............................

Abb. 2-21: Rastpolkurve....................................................................................................... 41

Abb. 2-22: Richtungskomponenten........................................................................................ 42

Abb. 2-23: Drehwinkel-Drehmoment-Kennlinie (Alpha(T)-Diagramm) ............................... 42

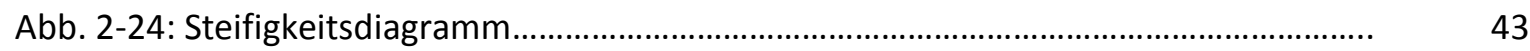

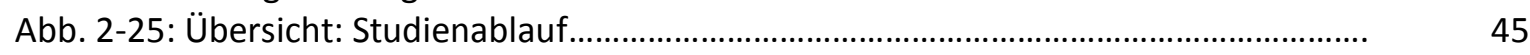

Abb. 2-26: Übersicht: Ablauf der Versuche............................................................................... 46

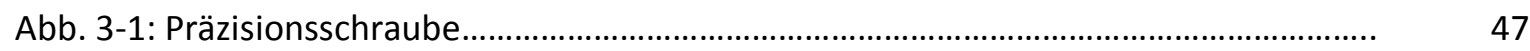

Abb. 3-2: Rastpolkurve; Präzisionsschraube........................................................................

Abb. 3-3: Richtungskomponenten; Präzisionsschraube.......................................................

Abb. 3-4: Lineare Regression der Pumpzeiten ........................................................................ 48

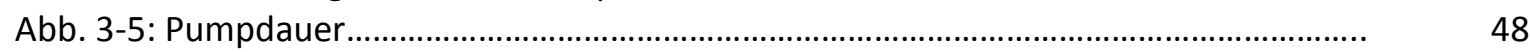

Abb. 3-6: Rastpolkurve; Bandscheibenmodell ohne Gelenke

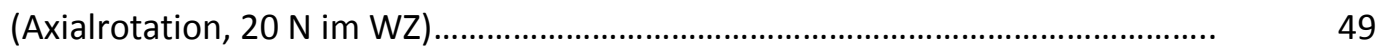

Abb. 3-7: Alpha(T)-Diagramm; Bandscheibenmodell ohne Gelenke......................................

Abb. 3-8: Richtungskomponenten; Bandscheibenmodell ohne Gelenke............................... $\quad 49$

Abb. 3-9: Rastpolkurve; Bandscheibenmodell mit Gelenken

(Axialrotation, $20 \mathrm{~N} \mathrm{im} \mathrm{WZ} \mathrm{und} 20 \mathrm{~mm}$ ventral des WZ)..................................... 50

Abb. 3-10: Rastpolkurve; isolierte Prestige ${ }^{\circledR}$ LP (Axialrotation, $60 \mathrm{~N}$ im WZ).........................

Abb. 3-11: Alpha(T)-Diagramm; isolierte Prestige ${ }^{\circledR}$ LP..............................................................

Abb. 3-12: Rastpolkurve; isolierte Prestige ${ }^{\circledR}$ LP mit Gelenken

(Axialrotation, $60 \mathrm{~N} \mathrm{im} \mathrm{WZ).............................................................................}$

Abb. 3-13: Richtungskomponenten; isolierte Prestige ${ }^{\circledR}$ LP mit Gelenken ............................

Abb. 3-14: Röntgenaufnahme; intaktes Segment 16/07 seitlich, Ausrichtung der IHA....... 53

Abb. 3-15: Rastpolkurve; intaktes Segment 10/07 (Axialrotation, 20N im WZ)

Linksrotation................................................................................................ 54

Abb. 3-16: Rastpolkurve; intaktes Segment 10/07 (Axialrotation, $20 \mathrm{~N} \mathrm{im} \mathrm{WZ)}$

Rechtsrotation.

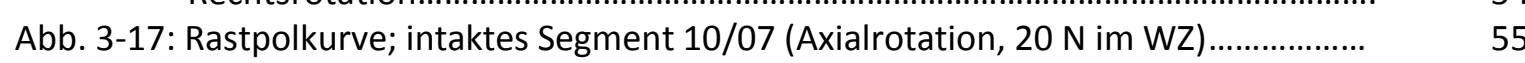

Abb. 3-18: Rastpolkurve; intaktes Segment 10/07 (Axialrotation, $60 \mathrm{~N} \mathrm{im} \mathrm{WZ)...................} 55$

Abb. 3-19: Rastpolkurve; intaktes Segment 10/07

(Axialrotation, 20 N 20 mm ventral des WZ).................................................... 56

Abb. 3-20: Rastpolkurve; intaktes Segment 10/07

(Axialrotation, $20 \mathrm{~N} 20$ mm dorsal des WZ) .................................................... 56

Abb. 3-21: Rastpolkurve; intaktes Segment 10/07

(Axialrotation, $20 \mathrm{~N} 15 \mathrm{~mm}$ lateral rechts des WZ)............................................ 56

Abb. 3-22: 3D-Rekonstruktion der Rastpolkurve; intaktes Segment 10/07

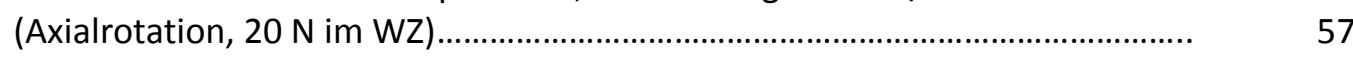

Abb. 3-23: Richtungskomponenten; intaktes Segment 10/07

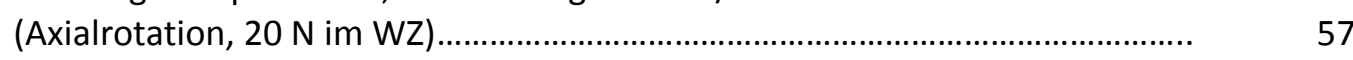

Abb. 3-24: Alpha(T)-Diagramm; intaktes Segment 10/07

(Axialrotation, $20 \mathrm{~N}$ und $60 \mathrm{~N}$ im WZ) ............................................................ 58

Abb. 3-25: Steifigkeitsdiagramm; intaktes Segment 10/07

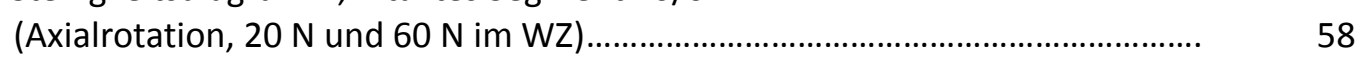

Abb. 3-26: Alpha(T)-Diagramm; intaktes Segment 10/07

(Axialrotation, $20 \mathrm{~N} \mathrm{im} \mathrm{WZ} \mathrm{und} 20 \mathrm{~mm}$ ventral und dorsal des WZ)................. 59

Abb. 3-27: Steifigkeitsdiagramm; intaktes Segment 10/07

(Axialrotation, $20 \mathrm{~N} \mathrm{im} \mathrm{WZ} \mathrm{und} 20 \mathrm{~mm}$ ventral und dorsal des WZ).................. 59

Abb. 3-28: Rastpolkurve; intaktes Segment 10/07

(Axialrotation, $20 \mathrm{~N} \mathrm{im} \mathrm{WZ} \mathrm{und} 20 \mathrm{~mm}$ ventral und dorsal des WZ) 
Abb. 3-29: Rastpolkurve; Prestige ${ }^{\circledR}$ LP 10/07

(Axialrotation, $20 \mathrm{~N}$ im WZ und $20 \mathrm{~mm}$ ventral und dorsal des WZ)

Seite

Abb. 3-30: Rastpolkurve; $\operatorname{Bryan}^{\circledR}$ 10/07

(Axialrotation, $20 \mathrm{~N}$ im WZ und $20 \mathrm{~mm}$ ventral und dorsal des $\mathrm{WZ}$ )

Abb. 3-31: Rastpolkurve; Präparat 10-07, $20 \mathrm{~N}$ im WZ

(Axialrotation, intaktes Segment, Prestige ${ }^{\circledR}$ LP und Bryan ${ }^{\circledR}$ )

Abb. 3-32: Teilrastpolkurven, Präparat 10/07, 20 N im WZ

(Axialrotation, intaktes Segment, Prestige ${ }^{\circledR}$ LP und Bryan ${ }^{\circledR}$ )

Abb. 3-33: Röntgenaufnahme seitlich, intaktes Segment, Prestige ${ }^{\circledR}$ LP und Bryan ${ }^{\circledR}$ 16/07; initiale Ausrichtung der IHA (Axialrotation)

Abb. 3-34: Versatz der initialen Rotationsachse (Axialrotation).

Abb. 3-35: Initiale Migrationsrate bei axialer Rotation in Abhängigkeit der Vorlastposition.

Abb. 3-36: Steifigkeitsdiagramm; intaktes Segment, Prestige ${ }^{\circledR}$ LP und Bryan ${ }^{\circledR}$ Cervical Disc 10/07 (Axialrotation, $20 \mathrm{~N}$ im WZ)...

Abb. 3-37: Alpha(T)-Diagramm; intaktes Segment, Prestige ${ }^{\circledR}$ LP und Bryan ${ }^{\circledR}$ Cervical Disc 10/07 (Axialrotation, $20 \mathrm{~N}$ im WZ).....

Abb. 3-38: Rastpolkurve; intaktes Segment 10/07 (Lateralflexion, $20 \mathrm{~N}$ im WZ).

Abb. 3-39: Rastpolkurve; intaktes Segment 10/07

(Lateralflexion, $20 \mathrm{~N} \mathrm{im} \mathrm{WZ} \mathrm{und} 20 \mathrm{~mm}$ ventral und dorsal des WZ).

Abb. 3-40: Rastpolkurve; Präparat 10/07, 20 N im WZ

(Lateralflexion, intaktes Segment, Prestige ${ }^{\circledR}$ LP und Bryan ${ }^{\circledR}$ Cervical Disc).......

Abb. 3-41: Richtungskomponenten; intaktes Segment 10/07

(Lateralflexion, $20 \mathrm{~N}$ im WZ).

Abb. 3-42: Röntgenaufnahme seitlich, intaktes Segment, Prestige ${ }^{\circledR}$ LP und

Bryan $^{\circledR} 16 / 07$; initiale Ausrichtung der IHA (Lateralflexion)....

Abb. 3-43: Steifigkeitsdiagramm; intaktes Segment, Prestige ${ }^{\circledR}$ LP und

Bryan ${ }^{\circledR}$ Cervical Disc 10/07 (Lateralflexion, $20 \mathrm{~N}$ im WZ)

Abb. 3-44: Alpha(T)-Diagramm; intaktes Segment, Prestige ${ }^{\circledR}$ LP und Bryan ${ }^{\circledR}$ Cervical Disc 10/07 (Lateralflexion, $20 \mathrm{~N}$ im WZ)

Abb. 3-45: Übersicht über die Entwicklung der initialen Steifigkeit in

Abhängigkeit von der sagittalen Vorlastposition

Abb. 3-46: Rastpolkurve; intaktes Segment 10/07

(Ventralflexion-Extension, $20 \mathrm{~N}$ im WZ).

Abb. 3-47: Rastpolkurve; intaktes Segment 10/07 (Ventralflexion-Extension, $20 \mathrm{~N}$ im WZ und $20 \mathrm{~mm}$ ventral und dorsal des WZ).

Abb. 3-48: Rastpolkurve; Präparat 10/07, $20 \mathrm{~N}$ im WZ (Ventralflexion-Extension, intaktes Segment, Prestige ${ }^{\circledR}$ LP und Bryan ${ }^{\circledR}$ Cervical Disc).

Abb. 3-49: Richtungskomponenten; intaktes Segment 10/07 (Ventralflexion-Extension, $20 \mathrm{~N}$ im WZ).

Abb. 3-50: Röntgenaufnahme; intaktes Segment 16/07 ap, Ausrichtung der IHA (Ventralflexion-Extension)

Abb. 3-51: Steifigkeitsdiagramm; intaktes Segment, Prestige ${ }^{\circledR}$ LP und Bryan ${ }^{\circledR}$ Cervical Disc 10/07 (Ventralflexion-Extension, $20 \mathrm{~N}$ im WZ)

Abb. 3-52: Alpha(T)-Diagramm; intaktes Segment, Prestige ${ }^{\circledR}$ LP und Bryan ${ }^{\circledR}$ Cervical Disc 10/07 (Ventralflexion-Extension, $20 \mathrm{~N} \mathrm{im} \mathrm{WZ)}$

Abb. 4-1: Röntgenaufnahme, seitlich, Position des C3-Wirbels vor und nach TDA. 
Tab. 1-1: Indikationen und Kontraindikationen für die Implantation zervikaler Bandscheibenprothesen.

Tab. 2-1: Übersicht Versuchsobjekte.

Tab. 3-1: Ergebnisse Präzisionsschraube

Tab. 3-2: Ergebnisse des reinen Bandscheibenmodells...

Tab. 3-3: Position der Widerstandszentren der acht Versuchsobjekte...

Tab. 3-4: Übersicht Steifigkeit und ROM; intaktes Segment

(Axialrotation, 20 und $60 \mathrm{~N}$ im WZ).

Tab. 3-5: Übersicht Steifigkeit und ROM; intaktes Segment

(Axialrotation, $20 \mathrm{~N} \mathrm{im} \mathrm{WZ} \mathrm{und} 20 \mathrm{~mm}$ ventral und dorsal des WZ)

Tab. 3-6: Durchschnittliches Migrationsausmaß der IHA vor und nach TDA

(Axialrotation)

Tab. 3-7: Neigungswinkel der initialen IHA zur Horizontalebene vor und nach TDA (Axialrotation)

Tab. 3-8: Differenz der Rotationsachsenposition unter verschiedenen Vorlastpositionen

(Axialrotation)

Tab. 3-9: Koordinaten des Schwerpunktes für Rechtsrotation (20 N im WZ)...

Tab. 3-10: Übersicht ROM; intaktes Segment, Prestige ${ }^{\circledR}$ LP und Bryan ${ }^{\circledR}$ Cervical Disc

(Axiale Rotation, $20 \mathrm{~N} \mathrm{im} \mathrm{WZ} \mathrm{und} 20 \mathrm{~mm}$ ventral und dorsal des WZ)

Tab. 3-11: Übersicht Steifigkeit; intaktes Segment, Prestige ${ }^{\circledR}$ LP und Bryan ${ }^{\circledR}$ Cervical

Disc (Axiale Rotation, $20 \mathrm{~N} \mathrm{im} \mathrm{WZ} \mathrm{und} 20 \mathrm{~mm}$ ventral und dorsal des WZ).........

Tab. 3-12: Vergleich der relativen Steifigkeit nach Prothesenimplantation

mit dem intakten Segment.

Tab. 3-13: Neigungswinkel der initialen IHA zur Horizontalebene vor und nach TDA

(Lateralflexion).

Tab. 3-14: Übersicht Steifigkeit; intaktes Segment, Prestige ${ }^{\circledR}$ LP und Bryan ${ }^{\circledast}$ Cervical Disc

(Lateralflexion, $20 \mathrm{~N} \mathrm{im} \mathrm{WZ} \mathrm{und} 20 \mathrm{~mm}$ ventral und dorsal des WZ)

Tab. 3-15: Übersicht ROM; intaktes Segment, Prestige ${ }^{\circledR}$ LP und Bryan ${ }^{\circledR}$ Cervical Disc

(Lateralflexion, $20 \mathrm{~N} \mathrm{im} \mathrm{WZ} \mathrm{und} 20 \mathrm{~mm}$ ventral und dorsal des WZ).

Tab. 3-16: Neigungswinkel der initialen IHA zur Transversalebene vor und nach TDA

(Ventralflexion-Extension).

Tab. 3-17: Übersicht Steifigkeit; intaktes Segment, Prestige ${ }^{\circledR}$ LP und

Bryan ${ }^{\circledR}$ Cervical Disc (Ventralflexion-Extension, $20 \mathrm{~N}$ im WZ und $20 \mathrm{~mm}$ ventral und dorsal des WZ).

Tab. 3-18: Übersicht ROM; intaktes Segment, Prestige ${ }^{\circledR}$ LP und Bryan ${ }^{\circledR}$ Cervical Disc (Ventralflexion-Extension, $20 \mathrm{~N}$ im WZ und $20 \mathrm{~mm}$ ventral und dorsal des WZ).

Tab. 3-19: Übersicht über die Maße der Facettengelenksflächen 


\section{Einführung}

\subsection{Einleitung}

Rücken- oder Nackenschmerzen stellen eine der häufigsten Beschwerdeformen in der Gesamtbevölkerung dar. Jährlich klagen in Deutschland mehr als $70 \%$ der Erwachsenen über Rückenschmerzen (Wenig et al. 2008). Die Ursachen sind vielfältig und umfassen sowohl akute Ereignisse, als auch chronische Schäden (Grifka et al. 1998, Schmitt et al. 2003). Oftmals gehen die Beschwerden mit morphologischen Veränderungen im Sinne einer Degeneration oder traumatischen Schädigung der Bandscheiben einher. Häufig handelt es sich dabei um junge Patienten (Chung et al. 2009). Neben individuellen Einschränkungen und Problemen verursachen Rückenschmerzen eine nicht unerhebliche, wirtschaftliche Belastung. 2008 wurden die jährlich verursachten Kosten in der Bundesrepublik Deutschland auf $1322 €$ pro Patient/-in geschätzt (Wenig et al. 2008). Dabei wurden etwa $46 \%$ durch die medizinische Versorgung und Therapie und ca. 54\% durch damit einhergehende Produktionsausfälle verursacht. Darüber hinaus gehören Rückenschmerzen zu den häufigsten Ursachen von Frühpensionierung (Niethard und Pfeil 2005).

Je nach Symptomatik, Verlauf und Schweregrad der Beschwerden erfolgt die Therapie konservativ oder operativ (Niethard und Pfeil 2005, Koller 2011, Grimmer und Wurm 2011). Dabei wird eine konservative Therapie (z.B.: Analgesie, Muskelrelaxation, Physiotherapie, Wärmeanwendung) primär bei muskulären Verspannungen bzw. Zerrungen und Bandscheibenvorfällen ohne neurologische Ausfälle angestrebt (Niethard und Pfeil 2005, Kluba und Roetman 2005a, Koller 2011, Grimmer und Wurm 2011).

Ein operatives Vorgehen ist hingegen bei degenerativen Veränderungen der Bandscheibe, Versagen der konservativen Therapie und Vorhandensein eines morphologischen Korrelats gerechtfertigt (Mehren und Mayer 2005).

Wenn eine Pathologie im Bereich der Bandscheibe bildmorphologisch gesichert ist und die entsprechenden Symptome vorliegen, so dass die Indikation zur Operation gestellt wird, stehen derzeit zwei mögliche Vorgehensweisen zur Verfügung. Einerseits die Fusion, das heißt die vollständige Entfernung der Bandscheibe mit anschließender Versteifung des betroffenen Bewegungssegmentes (Cloward 1958, Smith und Robinson 1958), welche weiterhin den Goldstandard darstellt (Habela und McAfee 2011, McKenzie 2011). Andererseits der Ersatz der geschädigten Bandscheibe durch ein künstliches Implantat („Total Disc Replacement“; TDR).

Trotz der langen und häufigen Anwendung werden immer wieder Probleme in Folge der Fusion eines Wirbelsäulensegmentes beobachtet. Durch eine Änderung der Biomechanik und der daraus resultierenden Überlastung werden die benachbarten Segmente übermäßig belastet und zeigen einen vorzeitigen Verschleiß („Adjacent Segment Disease“; ASD) (Bohlman et al. 1993, Goffin et al. 
1995, Hilibrand et al. 1999, Eck et al. 2002, Cunningham et al. 2003, Anderson und Rouleau 2004, Bertagnoli et al. 2006, Fritsch und Pitzen 2006). Darüber hinaus kann es zur Entstehung eines Falschgelenkes (Pseudarthrose) und zu Problemen bei der Verwendung eines Beckenkammspans (Entnahmemorbidität) kommen (Fritsch und Pitzen 2006).

Demgegenüber wird auch der Einsatz von Bandscheibenprothesen (TDR) kontrovers diskutiert. Zwar nimmt die Zahl der Implantationen von Jahr zu Jahr zu, jedoch liegen nur wenig, zum Teil sogar überhaupt keine biomechanischen Daten zu den Implantaten vor (Fritsch und Pitzen 2006, Bartels et al. 2008, Chung et al. 2009). Dennoch verspricht man sich durch die Implantation einer TDR die Vermeidung der ASD (Anderson et al. 2004, Dmitriev et al. 2005, Chang et al. 2007, Bartels et al. 2008, Chung et al. 2009), den Erhalt des physiologischen Bewegungsausmaßes („Range of motion“; ROM) (Puttlitz et al. 2004) und des intradiskalen Druckes der angrenzenden Segmente (Chang et al. 2007). Zudem existieren bereits sehr gute klinische, mit der Fusion vergleichbare Kurzzeitergebnisse (Gibson und Waddell 2005, Freeman und Davenport 2006). Kritiker des künstlichen Bandscheibenersatzes behaupten jedoch, dass die ASD nach Fusion nie eindeutig bewiesen worden sei (Villas et al. 1994, Beguiristain et al. 1994, Fritsch und Pitzen 2006, Bartels et al. 2008). Es gäbe zudem keine Studien, die eine Überlegenheit der TDR gegenüber der Fusion zeigen (Freeman und Davenport 2006) und ebenso wenig Langzeitergebnisse die beweisen, dass unter TDR die Entstehung der ASD vermieden werde (Gibson und Waddell 2005, Freeman und Davenport 2006).

Zusammenfassend kann somit festgehalten werden, dass nur sehr wenige, oder gar keine Daten zur Biomechanik der mit einer TDR versorgten Segmente existieren. Außerdem kann die Grundproblematik der veränderten Biomechanik angrenzender Segmente und die daraus resultierende Degeneration der Anschluss-Segmente (ASD) nicht eindeutig bewiesen werden. Die ASD stellt dabei das wichtigste Argument der Befürworter der Bandscheibenprothesen (TDR) dar.

\section{$\underline{\text { Stand der biomechanischen Forschung der Wirbelsäule }}$}

Um die mechanische Belastung insbesondere der Gelenkfacetten während physiologischer Bewegungen zu bewerten, ist es notwendig, die Segmentkinematik, also die Änderung von Lage und Ausrichtung der momentanen Rotations- bzw. Schraubachse, in differentiell kleinen Abständen experimentell zu ermitteln. In den meisten Untersuchungen werden die Messungen segmental durchgeführt. Dabei besteht ein Bewegungssegment („Junghanns'sches Segment“) aus zwei benachbarten Wirbeln, die über Bänder, die Bandscheibe und die beiden Wirbelbogengelenke gelenkig miteinander verbunden sind. In der Regel wird auf den oberen Wirbelkörper ein zeitveränderliches Kraftsystem ausgeübt, welches aus einem Drehmoment und/oder einer axialen Kraft besteht. Daraus ergeben sich Relativbewegungen des oberen Wirbelkörpers, die durch sogenannte Drehwinkel-Drehmoment-Kennlinien bewertet werden und das Bewegungsausmaß 
(ROM) und die Steifigkeit erhoben werden. Jedoch reicht die Ermittlung dieser Parameter für eine biomechanische Analyse der Segmentkinematik, besonders im Hinblick auf die Evaluation bewegungserhaltender Implantate, nicht aus (Fritsch und Pitzen 2006, Bartels et al. 2008, Chung et al. 2009), sodass zunehmend Bemühungen unternommen wurden die Lage und Ausrichtung der Rotationsachse zu bestimmen. Dabei gingen einige Wissenschaftler zunächst von einer ortsfesten „helical axis of motion“ (HAM) aus, um welche sich der bewegte Wirbel für das gesamte Bewegungsausmaß (ROM) in einer schraubenförmigen Bewegung bewege. Die Berechnung der HAM erfordert lediglich zwei Positionen des bewegten Körpers, deren Unterschied etwa dem ROM entspricht (Cossette et al. 1971, Haberl et al. 2004, Niosi et al. 2006, Oxland et al. 1994, Panjabi et al. 1994, White und Panjabi 1978, Zhu et al. 2007). Der Abstand beider Positionen war in diesen Studien jedoch nicht differentiell klein (Nägerl et al. 1995), sodass andere Arbeitsgruppen begannen, den ROM in mehrere Intervalle zu unterteilen und für jeden Abschnitt die Schraubachse („Finite Helical Axis"; FHA) zu berechnen (Kettler et al. 2004, Rousseau et al. 2006). Es zeigten sich klare Unterschiede in der räumlichen Lage der FHA. Damit wurde zwar qualitativ nachgewiesen, dass während der Bewegung eines Segmentes die momentane Schraubachse („Instantaneous Helical Axis"; IHA) wandert, die tatsächliche Segmentkinematik wurde jedoch noch nicht hinreichend erfasst. Nach den Gesetzen der räumlichen Kinematik (Wolf 1947) wird der Übergang von einer Position in eine andere nur dann eindeutig beschrieben, wenn die beiden Positionen 1 und 2 differentiell benachbart sind. Die Differenzen $\Delta \alpha$ (Änderung des Rotationswinkels) und $\Delta \mathrm{s}$ (Änderung des AchsenAbstandes) müssen demnach gegen Null gehen und so zu kleinen Differentialen da und ds entarten. In den letzten Jahren wird mit der computergestützten Simulations-Berechnung („Finite Element Method“; FEM) ein neuartiger Ansatz der biomechanischen Analyse der Wirbelsäule verfolgt. Im Rahmen dieser Methode wird mit großem Aufwand die Bandscheibe mit Nucleus pulposus und Anulus fibrosus virtuell modelliert. Allerdings werden die Wirbelbogengelenke nicht berücksichtigt oder werden nur als einfache Plattenführungen angenommen (Bono und Lee 2004, Schmidt et al. 2008a, Schmidt et al. 2008b, Rohlmann et al. 2009), weshalb die Gelenkführung, die durch die Krümmungsmorphologie der Gelenkflächen (Nägerl et al. 1995, Wachowski et al. 2009a) die Segmentkinematik bestimmt (Wachowski et al. 2009a, Wachowski et al. 2009b), in den Berechnungen völlig außer Acht gelassen wird. Daher ist die Validität und Wertigkeit dieser Rechnungen sehr kritisch zu hinterfragen.

Um den tatsächlichen Bewegungsvorgang aufzulösen, wurde in dieser Studie eine auf Induktionstastern basierende 6D-Messapparatur verwendet. Dabei konnte eine räumliche Auflösung des bewegten Wirbelkörpers von $<2,4 \mu \mathrm{m}$ für Translation und $<1$ mdeg für Rotation erreicht werden. Die zeitliche Auflösung betrug etwa 300 bis 400 Positionsbestimmungen pro ROM in der Halswirbelsäule und ca. 800 in der Lendenwirbelsäule. Für jedes einzelne Intervall konnte die Lage 
der FHA berechnet werden. Bei der erreichten Auflösung kann postuliert werden, dass die FHA-Lagen die entsprechende Wanderung der IHA in guter Näherung widerspiegeln. Somit konnten die Position und Ausrichtung der IHA praktisch differentiell bestimmt werden und die tatsächliche Kinematik des Segmentes erfasst werden (Mansour et al. 2004, Wachowski et al. 2007, Wachowski et al. 2009a, Wachowski et al. 2009b).

\subsection{Anatomische Grundlagen}

\subsubsection{Anatomie der Wirbelsäule}

Die Wirbelsäule (Columna vertebralis) ist aus 24 einzelnen Knochen, die über 23 Synchondrosen miteinander verbunden sind, aufgebaut und gliedert sich in fünf Abschnitte (Abb. 1-1). Die Halswirbelsäule (HWS) setzt sich aus den sieben Halswirbeln (Vertebrae cervicales) C1 bis C7 zusammen und steht über den Atlas (1. Wirbel) mit der Schädelbasis in Verbindung. Die angrenzende Brustwirbelsäule (Pars thoracalis) ist aus zwölf Wirbeln aufgebaut (Th1 bis Th12). Die Lendenwirbelsäule (LWS) umfasst fünf Lendenwirbeln (L1 bis L5) und geht in das Kreuzbein (Os sacrum) über. Dieses besteht wiederum aus fünf Wirbeln, die jedoch miteinander verschmolzen sind. Den Abschluss bildet das Steißbein (Os coccygis) mit drei bis
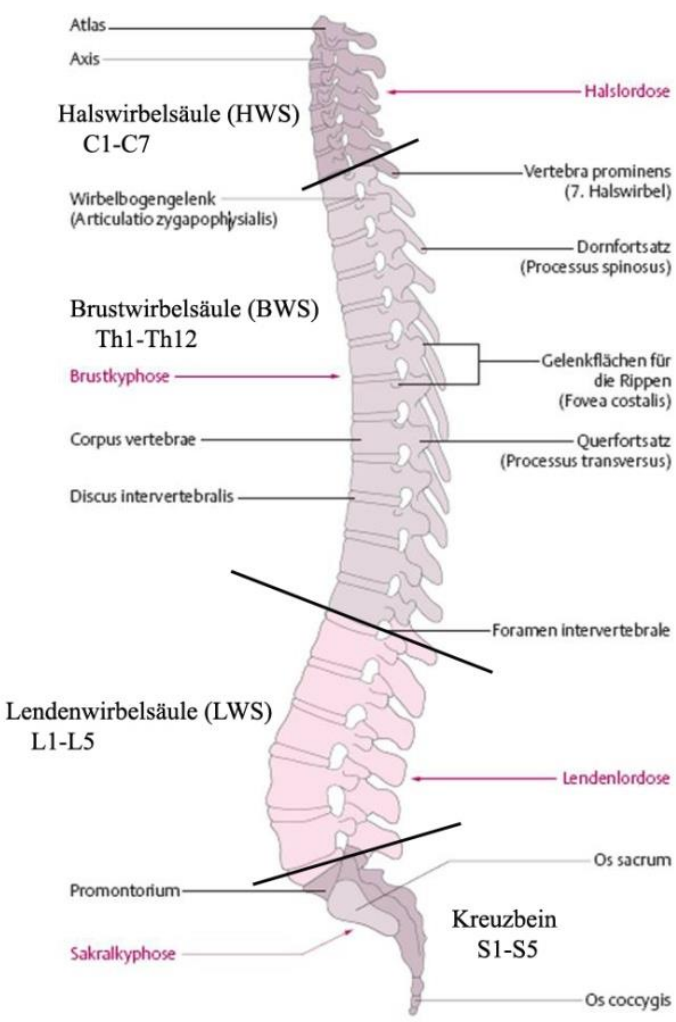

Abb. 1-1: Einteilung der Wirbelsäule (modifiziert nach Bommas-Ebert et al. 2006, S. 154) fünf rudimentären Steißwirbeln (Co1 - Co5).

Zwischen den aneinander grenzenden Wirbeln (von C2 bis S1) befinden sich die Zwischenwirbelscheiben, welche die Columna vertebralis gemeinsam mit den Wirbelbogengelenken und der angreifenden Bandapparatur zu einer funktionellen Einheit verbinden. Sie haben zusätzlich einen dämpfenden Effekt bei axialer Krafteinwirkung. Der Abfederung von einwirkenden Kräften kommt darüber hinaus die charakteristische „Doppel-S-Krümmung“ der Wirbelsäule zugute. Im Bereich der HWS und LWS liegt dabei eine nach vorn konvexe Krümmung (Lordose; „vorwärts gekrümmt") vor, während der Abschnitt der Brustwirbelsäule und das Steißbein eine Konvexität nach hinten (Kyphose; „gebückt“) aufweisen. Darüber hinaus erfüllt die Columna vertebralis durch die knöcherne Ummantelung von Rückenmark (Medulla spinalis) und Spinalnerven eine weitere Schutzfunktion. 
Das Bewegungsausmaß der Wirbelsäule beruht auf der Beweglichkeit der einzelnen Wirbel untereinander und entspricht daher der Summe aller Teilbewegungen. Die kleinste funktionelle Einheit stellt das von Junghanns 1977 beschriebene „Junghanns'sche Segment“ dar. Es setzt sich aus zwei aneinandergrenzenden Wirbeln, der eingeschlossenen Zwischenwirbelscheibe und dem fixierenden Bandapparat zusammen.

\subsubsection{Wirbel}

Wie auch in den übrigen Teilen der Wirbelsäule artikulieren im Bereich der HWS benachbarte Wirbel über die Wirbelbogengelenke (Artt. zygapophysiales) mit einander, die durch die Procc. articulares (s. Abb. 1-2) gebildet werden. Mit den Uncovertebralgelenken verfügt die Halswirbelsäule, verglichen mit den anderen Abschnitten, über eine weitere gelenkige Verbindung (Abb. 1-3). Sie sind nicht primär angelegt, sondern entstehen sekundär. In der Kindheit kommt es zunehmend zu einer Anhebung der anfänglich flach ausgerichteten Processus uncinati (Unci corporis, Hakenfortsätze) (Platzer 1999b). Infolgedessen besteht eine Konkavität der Wirbelkörperdeckfläche in transversaler Richtung. Die Grundplatte des Wirbels ist hingegen nach sagittal konkav (Putz 1981). In kaudaler Richtung nimmt die

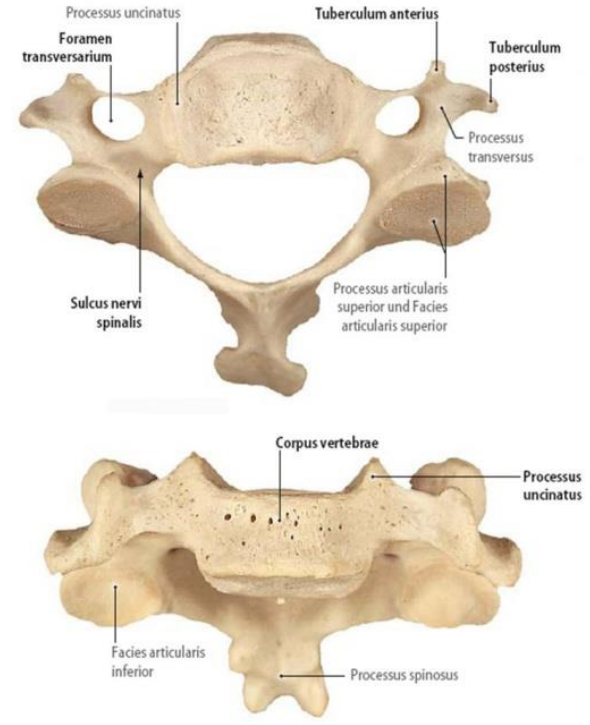

Abb. 1-2: Anatomie des Halswirbels; oben: 4. Halswirbel von oben; unten: 6 . Halswirbel von ventral (modifiziert nach Tillmann 2005, S. 193) Ausprägung dieser Strukturbesonderheit ab. Einen wichtigen Anteil im Aufbau der Uncovertebralgelenke stellen die Zwischenwirbelscheiben dar. Diese bestehen in der HWS zum größten Teil aus einem robusten Nucleus pulposus (Mercer und Bogduk 1999), der nur ventral der Procc. uncinati sichelförmig von einem echten Anulus fibrosus begrenzt ist (Mercer und Bogduk 1999, Tonetti et al. 2005). Auf Höhe der Processus uncinati besteht der Anulus fibrosus aus faszienartigem Bindegewebe. Kranial und kaudal geht dieses in das Periost über, ventral und dorsal ist es mit den Längsbändern verwachsen. Bei einem Bandscheibenvorfall präsentiert sich der Übergang in die Ligamenta longitudinalia oft als Schwachstelle, wodurch es hier zu einem Durchtritt von Bandscheibengewebe in den Spinalkanal kommen kann und die Gefahr einer Einklemmung des Rückenmarks besteht (Tanaka et al. 2000). Dorsal der Procc. uncinati wird der Nucleus pulposus letztlich von einer Schicht aus senkrechten Kollagenfasern begrenzt. 
Zwischen dem 5. und 10. Lebensjahr treten infolge der Bewegung Spaltbildungen im Knorpel auf, welche in der 2. bis 3. Lebensdekade die gesamte Bandscheibe durchziehen können (Luschka 1856, Töndury 1955, Töndury und Theiler 1990). In der Folge resultiert eine metaplastische Umwandlung des umgebenden
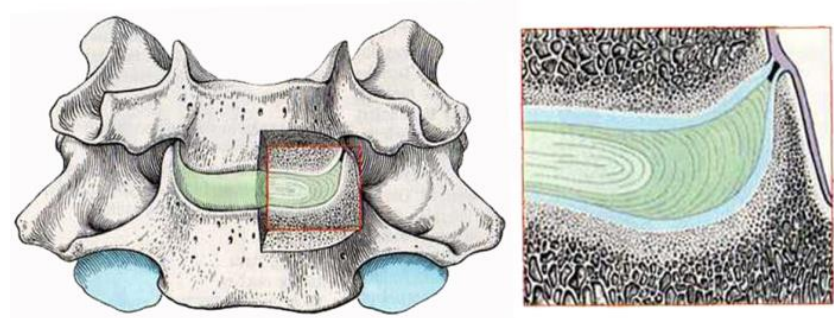

Abb. 1-3: Frontale Ansicht eines HWS-Segmentes (links) und vergrößerte Darstellung des Bereiches der Faserknorpels zu hyalinem Knorpel (Köster 1985). Durch das Einsprossen von Bindegewebe entstehen meniskoide Falten (Töndury und Theiler 1990), wodurch sich ein gutes Gleitlager für die Uncovertebralgelenke bildet.

\subsubsection{Wirbelbogengelenke}

Die Wirbelbogengelenke (Articulationes zygapophysiales) werden auch als Facettengelenke oder kleine Wirbelgelenke bezeichnet und setzen sich aus den Gelenkfortsätzen zweier benachbarter Wirbel zu einem synovialen Gelenk zusammen (Abb. 1-4). Die umliegende Gelenkkapsel ist im kranialen Anteil der Wirbelsäule locker und nimmt nach kaudal an Straffheit zu (Platzer 1999b).

Im Halsbereich findet man meniskusähnliche Einlagerungen (Plicae synoviales), die eine erhöhte Belastungsfähigkeit ermöglichen (Platzer 1999b). Mechanorezeptoren in den meniskoidalen Falten erfassen kontinuierlich die Lage der aneinandergrenzenden Wirbel, sowie die Kapselspannung und spielen daher eine wichtige Rolle in der neuromuskulären Steuerung
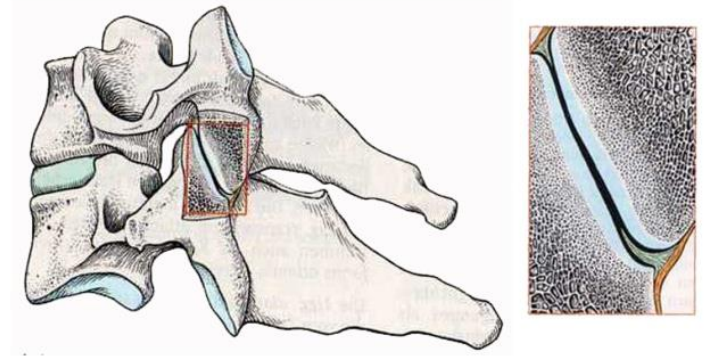

Abb. 1-4: Seitliche Darstellung eines HWSSegmentes (links) und vergrößerte Darstellung eines Facettengelenks (rechts) (Platzer 1999c, S. 59) (Ohtori et al. 2001, Lu et al. 2005). Die Ausrichtung der Gelenkfortsätze verändert sich in den einzelnen Abschnitten im Laufe der Entwicklung. Wahrscheinlich liegt der Grund dafür in der Adaptation an die unterschiedliche Beweglichkeit in den jeweiligen Bereichen der Wirbelsäule (Lutz 1967, Reichmann 1971). Eine ausgeprägte Inkongruenz und die geringe Krümmung der Gelenkflächen unterscheiden die Articulationes zygapophysiales der HWS von denen, der anderen Abschnitte. Da die Krümmungsmittelpunkte der beteiligten Gelenkflächen unterschiedlich sind (Putz 1981), können sie während der Bewegung temporär keilförmig auseinander klaffen (Putz 2003). Ausgeprägte sichelförmige Synovialfalten im Bereich des ventralen Gelenkspaltes können als Hinweis auf die Krümmungsinkongruenz gewertet werden (Nägerl 1990). Der Gelenkspalt verläuft von 
ventro-kranial nach dorso-kaudal und ist auf Höhe $\mathrm{C} 3 / \mathrm{C} 4$ um $40^{\circ}$ zur Transversalebene geneigt. Die weite und schlaffe Gelenkkapsel verfügt über sogenannte Recessus und ermöglicht dadurch einen deutlichen Bewegungsspielraum. Mit einem Bewegungsausmaß von $53^{\circ}$ in Lateralflexion und Flexions-/Extensionsrichtung sowie einem axialen Rotationsumfang von ca. $85^{\circ}$ stellt die Halswirbelsäule den beweglichsten Abschnitt der Wirbelsäule dar.

\subsubsection{Zwischenwirbelscheiben}

Die Bandscheibe (Discus intervertebralis) ist ein bradytrophes Gewebe mit geringer Stoffwechselaktivität, die mittels Diffusion versorgt wird und mechanosensibel innerviert ist (Ohtori et al. 2001, Aoki et al. 2004). Sie stellt das Bindeglied zwischen zwei aneinander grenzende Wirbel dar. In kranio-kaudaler Richtung nehmen sowohl der Durchmesser als auch die Höhe der Zwischenwirbelscheiben zu (Farfan 1979). Im Bereich der Hals- und Lendenwirbelsäule ist ihr vorderer Anteil höher, der hintere hingegen abgeflacht. Im Brustbereich verhält es sich umgekehrt. Der Discus intervertebralis wird in einen äußeren Faserring (Anulus fibrosus) und einen inneren gallertigen Kern (Nucleus pulposus) eingeteilt (siehe Abb. 1-5). Der Anulus fibrosus ist aus 10 bis 20 konzentrisch angeordneten Kollagenlamellen (Bogduk und Twomey 1991) und einem Faserknorpelanteil aufgebaut. Die bindegewebigen Fasern einer Lamelle sind dabei so angeordnet, dass sie gleichmäßig schräg und gegenläufig zu den Fasern der benachbarten Lamelle verlaufen und ein zugfestes Netzwerk entsteht (Kapandji 1985). Die inneren Fasern gehen ohne scharfe Grenze in den Nucleus pulposus über. Die Proteoglykanmatrix des Kerns hat einen Wasseranteil von 90\% (Krämer 1987, Götz et al. 1997, Götz et al. 1999), was auf das Wasserbindungsvermögen der Glykosaminoglykane zurückzuführen ist. Dadurch entsteht ein hoher kolloidosmotischer Druck, der die Fasern des Anulus fibrosus unter Spannung setzt und maßgeblich am Verhalten des Bandscheibenkerns beteiligt (Töndury 1955). Seine Hauptaufgabe liegt in der gleichmäßigen Druckverteilung auf den angrenzenden Wirbel. Die axiale Belastung wird dabei teilweise auf den äußeren Faserring umgeleitet, wodurch die Lamellen unter Zug gesetzt werden. Dabei ist zu beachten, dass hauptsächlich der Wirbelkörper und nicht die Bandscheibe bei vertikaler Belastung komprimiert wird (McKenzie 2011). Der Nukleus behält dabei seine kugelige Form und die kribriformen Endplatten federn in den Wirbelkörper (Roaf 1960, Lindahl 1976). Unter dezentraler Krafteinwirkung, wie sie bei der Seitwärtsneigung entsteht, verlagert sich der Nucleus pulpousus zur Gegenseite (Krag et al. 1987, Fennel et al. 1996). Bei einer Rotationsbewegung kommt es durch die charakteristische Faseranordnung des Anulus fibrosus zu einer seitlichen Kompression und Verformung des Gallertkerns, was in einer Höhenzunahme der Bandscheibe resultiert (Nägerl et al. 1995). 


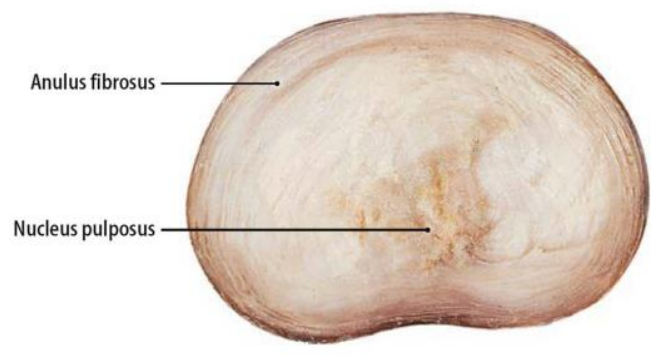

Abb. 1-5: Aufbau der Bandscheibe (Tillmann 2005, S. Abb. 1-6: Funktion der Bandscheibe; a) axialer Druck 201)

\subsubsection{Bandapparatur der Wirbelsäule}

Bänder (Ligamenta) verbinden bewegliche Elemente des Knochenskeletts und verhindern eine übermäßige Bewegung der Gelenke. Im Bereich der Wirbelsäule unterscheidet man sieben verschiedene Bänder (Abb. 17). Das vordere Längsband (Ligamentum longitudinale anterius) verläuft vom Atlas (erster Halswirbel) bis zum Kreuzbein, das hintere

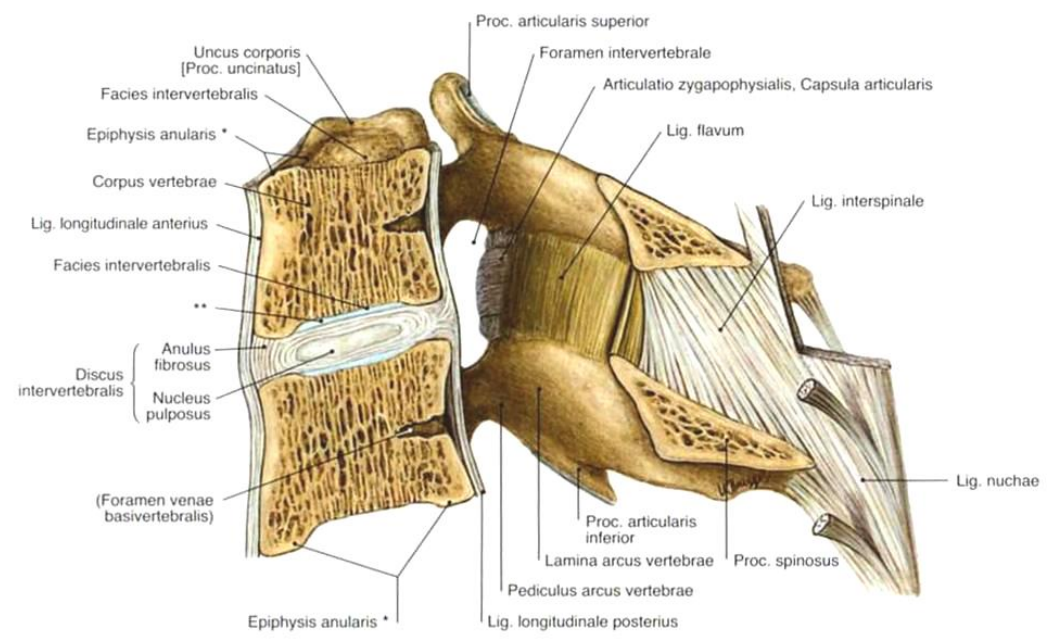

Abb. 1-7: Bandapparatur der Halswirbelsäule auf Höhe des 4. und 5. Halswirbels (Putz und Pabst 2004, S.25)

Längsband (Ligamentum longitudinale posterius) vom zweiten Halswirbel bis zum Discus intervertebralis zwischen den Lendenwirbeln L3/L4. Die Ligamenta flava verbinden die Wirbelbögen zweier benachbarter Wirbel und begrenzen somit den Wirbelkanal sowohl seitlich als auch dorsal. Das Ligamentum nuchae erstreckt sich von der Protuberantia occipitalis externa zu den Dornfortsätzen der Halswirbel. Es geht in die Ligamenta interspinalia bzw. das Ligamentum supraspinale über und dient als Ansatzpunkt der Rückenmuskulatur. Das Ligamentum supraspinale erstreckt sich über die Dornfortsätze bis zum Kreuzbein. Die Ligg. interspinalia spannen sich zwischen den Dornfortsätzen aneinander grenzender Wirbel auf und verhindern mit den Disci intervertebrales eine übermäßige Gleitbewegung der Wirbel und schränken mit dem Lig. supraspinale die Ventralflexion ein. Zwischen den Enden der Querfortsätze benachbarter Wirbel befinden sich die Ligamenta intertransversaria, welche die Lateralflexion und Axialrotation begrenzen. 


\subsubsection{Muskulatur der Wirbelsäule}

Die Muskulatur der Wirbelsäule ist nicht nur für deren Bewegung von großer Bedeutung, sondern hat auch einen entscheidenden Einfluss auf die Stabilität und Formsicherung des Rumpfes (Wurzinger 2007). Entsprechend dem entwicklungsgeschichtlichen Ursprung unterscheidet man im Halsbereich die tief liegende, autochthone oder primäre Rückenmuskulatur von der Gruppe der oberflächlich gelegenen und eingewanderten, sekundären Muskulatur (Platzer 1999b). Autochthon bedeutet, dass die Muskulatur nicht aus einer anderen Region des Körpers einwandert, sondern ursprünglich in diesem Bereich angelegt ist. Sie wird in ihrer Gesamtheit als Musculus erector spinae bezeichnet und durch die Rami dorsales der Spinalnerven innerviert. Entsprechend der Anordnung und Funktion kann die autochthone Rückenmuskulatur in einen medialen und lateralen Trakt und weiter in funktionelle Systeme eingeteilt werden (Abb. 1-9). Die kurze Nackenmuskulatur gehört teilweise zum medialen, teils zum lateralen Trakt. Die ventrale Halsmuskulatur wird von den Rami ventrales der Spinalnerven innerviert und ist aus einer oberflächlichen und tiefen Schicht aufgebaut (Abb. 1-8). Die Wirkung der einzelnen Muskeln lässt sich biomechanisch in Form einer resultierenden Kraft bei gleichzeitig wirkendem Drehmoment beschreiben (siehe biomechanische Grundlagen 1.5.2).

\section{Ventrale Halsmuskulatur}

Oberflächliche Schicht

Platysma

M. sternocleidomastoideus

Mm. infrahydoidei

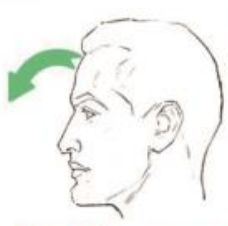

Kopfbeugung

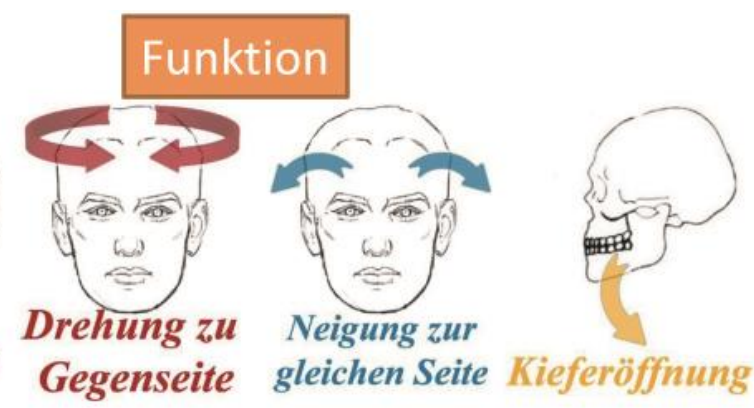

gleichen Seite Kieferöffnung
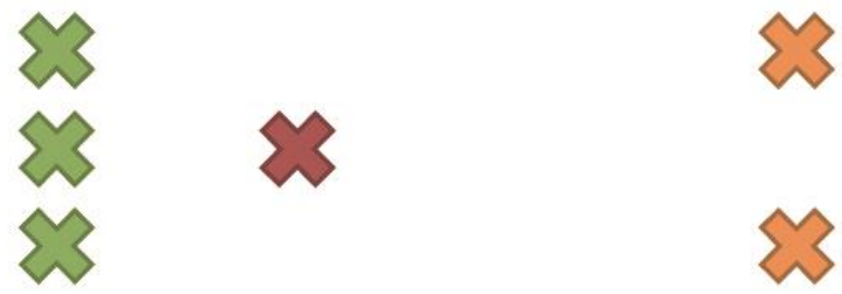

\section{Tiefe Schicht}

Mm. scaleni

Prävertebrale Halsmuskulatur
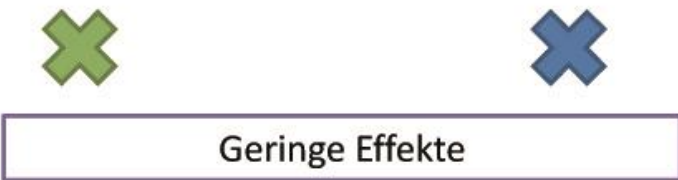

Abb. 1-8: Einteilung und Funktion der ventralen Halsmuskulatur (Abbildungen modifiziert nach Hartmann 2012) 

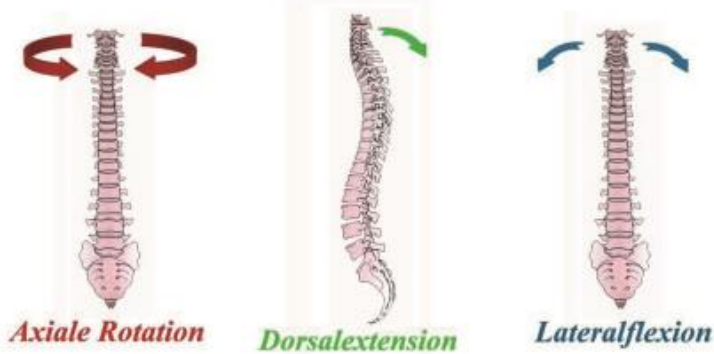

\section{Medialer Trakt}

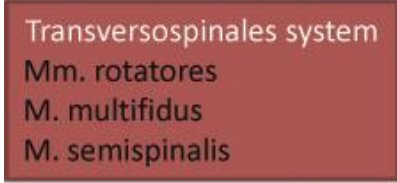

Spinales System

M. spinalis

$\mathrm{Mm}$. interspinales

\section{Lateraler Trakt}

Sakrospinales System

$\mathrm{M}$. longissimus

M. iliocostalis

Spinotransversales System

M. splenius capitis

M. splenius cervicis

Intertransversales System

$\mathrm{Mm}$. intertransversarii

\section{Kurze Nackenmuskulatur}
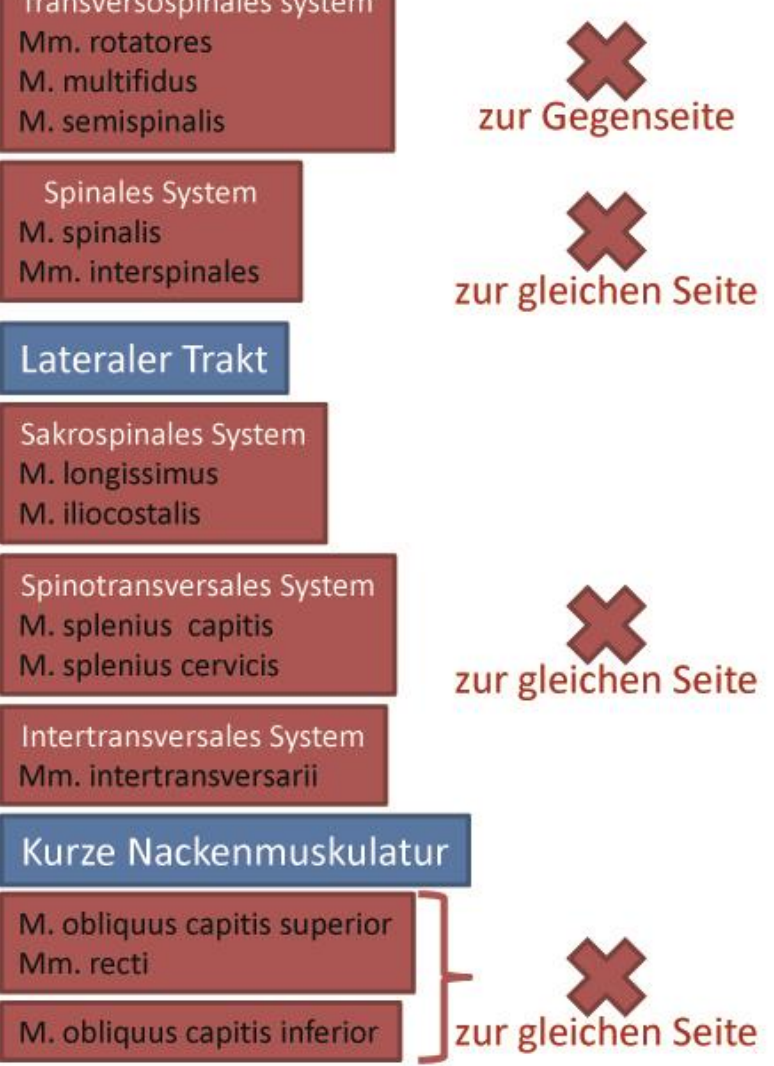

zur gleichen Seite

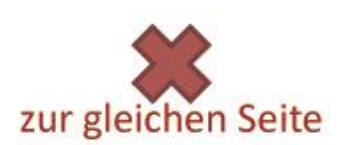

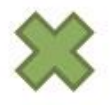
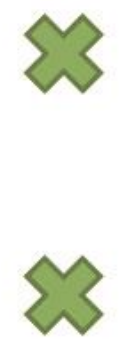

zur gleichen Seite

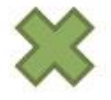
zur gleichen Seite

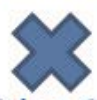

zu beiden Seiten

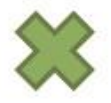

Abb. 1-9:

Einteilung und

Funktion der autochthonen Rückenmuskulatur (Abbildungen modifiziert nach Schadé 2001, S. 711)

zur gleichen Seite

\subsection{Pathologische Veränderungen der Halswirbelsäule}

Krankhafte Veränderungen im Bereich der Halswirbelsäule können mit einer Vielzahl an Symptomen einhergehen. Zu den häufigsten Beschwerden zählen Hals- und Nackenschmerzen (=HWS-Syndrom), Kopfschmerz, Myogelosen (Muskelverhärtung), Bewegungssteife und neurologische Ausfallerscheinungen (Parästhesien, Hypästhesien, Paresen) (Rössler und Rüther 2005, Niethard und Pfeil 2005, Reinhold et al. 2006, Leidel et al. 2008). Darüber hinaus können vegetative Symptome, wie Schwindel, Seitendifferenz der Hauttemperatur, Sehstörungen, Ohrgeräusche und Übelkeit auftreten. Dies liegt einerseits an der anatomischen Nähe der Arteria vertebralis, die durch die Foramina transversalia nach kranial läuft und von sympathischen Nervenfasern begleitet wird, 
andererseits enthalten die aus dem Rückenmark austretenden Nervenwurzeln ebenfalls vegetative Fasern, die durch degenerative Prozesse manipuliert werden können (Rieke 2008, Gumbert 2012a). Ein Rückschluss auf die Ätiologie ist anhand der Symptomkomplexe nur bedingt möglich. Im Folgenden werden pathologische Veränderungen beschrieben, welche zu den oben genannten Symptomen führen können und gleichzeitig eine Indikation zur TDA darstellen (siehe auch 1.4.3 Indikationen und Kontraindikationen).

\subsubsection{Degenerative Erkrankungen der Halswirbelsäule}

Im Laufe des Alterungsprozesses treten bei nahezu allen Menschen degenerative Veränderungen im Bereich der Halswirbelsäule auf (Kluba und Roetman 2005a). Aufgrund der guten Beweglichkeit und hohen Belastung sind die Verschleißerscheinungen im Hals- und Lendenbereich am stärksten ausgeprägt (Rössler und Rüther 2005). Klinisch relevant werden die Veränderungen jedoch erst, wenn sie vorzeitig auftreten, bei fortgeschrittenem Schweregrad oder sobald sie mit einer Beeinträchtigung von Sensibilität und Motorik einhergehen (Kluba und Roetman 2005a).

Aufgrund der axialen Druckbelastung kommt es zu einem Flüssigkeitsverlust des bradytrophen, nicht vaskularisierten Bandscheibengewebes. Dies führt im Verlauf eines Tages zu einer Höhenminderung der Bandscheibe. In waagerechter Position verringert sich hingegen der Druck, was wiederum zu einer Aufnahme von Flüssigkeit und Wiederherstellung der Pufferwirkung führt. Ab dem dritten Lebensjahrzehnt sinkt die Fähigkeit des Gallertkerns Wasser zu binden, was zu Rissen im Anulus fibrosus und einem Bandscheibenvorfall führen kann (Kluba und Roetman 2005a). In Abbildung 1-10 sind die verschiedenen Degenerationserscheinungen der Halswirbelsäule schematisch dargestellt.

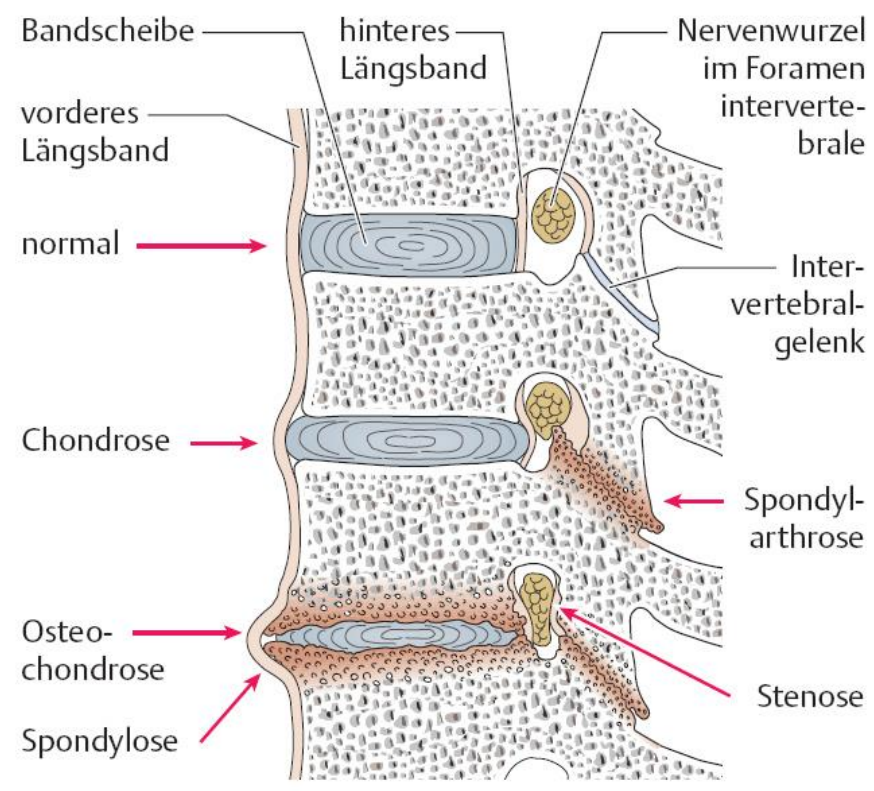

Abb. 1-10: Schematische Darstellung der Wirbelsäulendegenerationsarten. (Kluba und Roetman 2005b, S. 235) 
Bleibt es im Verlauf der Degeneration lediglich bei einer Höhenminderung der Zwischenwirbelscheiben, ohne Veränderung im Bereich der Wirbelkörper, spricht man von einer Chondrose (Knorpeldegeneration). Mit der Abnahme der Bandscheibenhöhe steigt der Druck auf die hinteren Bestandteile der Wirbelsäule und resultiert häufig in einem Verschleiß der Facettengelenke (Spondylarthrose). Darüber hinaus entwickelt sich häufig eine Sklerose der Grund- und Deckplatten, was als Osteochondrose bezeichnet wird. Degenerative Veränderungen können sich auch in Form von knöchernen Anbauten an den Wirbelgelenken oder Wirbelkörpern manifestieren (Spondylose) und zu einer Spinalkanalstenose führen.

\subsubsection{Zervikaler Bandscheibenvorfall}

Die Rissbildungen im Anulus fibrosus, die ab der dritten Lebensdekade regelmäßig auftreten können, sowie der Verschleiß des Bandscheibenmaterials im Rahmen des Alterungsprozesses, erhöhen das Risiko für einen Bandscheibenvorfall (Rössler und Rüther 2005). Je nach Schweregrad (Abb. 1-11) kann das klinische Bild sehr unterschiedlich ausfallen. Die Beweglichkeit der Halswirbelsäule ist meist eingeschränkt. Neben den oben genannten Symptomen kann es typischerweise zu Brachialgien (Armschmerzen), Dermatom-bezogenen Gefühlsstörungen, Muskellähmungen und wurzelspezifischen Reflexausfällen kommen (Kluba und Roetman 2005a). Die schwerwiegendste Komplikation stellt eine Kompression des Rückenmarks, bei einem Prolaps nach dorsal in den Wirbelkanal, dar. Ein Vorfall kann bei dezentraler, inkompletter Rückenmarkkompression zu einer „Halbseitensymptomatik“ (Missempfindung in einer Körperseite, Funktionsverlust der Extremitäten, Reflexdifferenz, Störungen der Bewegungskoordination) führen (Koller 2011). Eine vollständige Querschnittssymptomatik tritt bei einer mehr oder weniger kompletten Komprimierung auf.
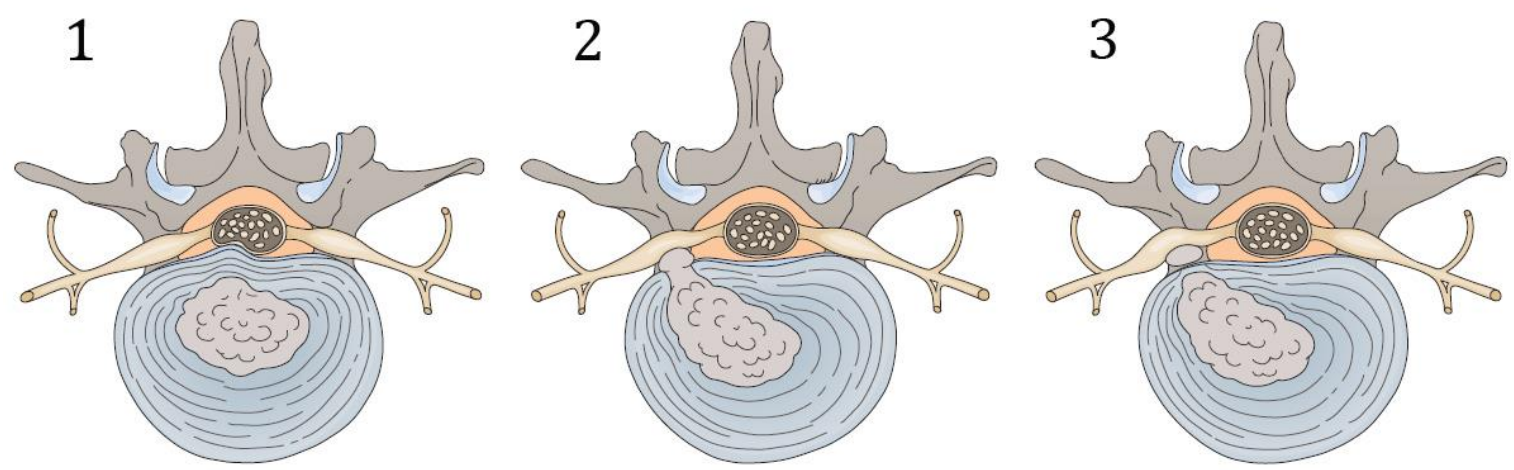

Abb. 1-11: Schweregradeinteilung der Bandscheibendegeneration. 1) Protrusion mit Vorwölbung des Gallertkerns innerhalb der Bandscheibe, 2) Bandscheibenprolaps oder -vorfall: Riss im Faserring und Austritt von Kernmaterial, 3) sequestrierter Vorfall: zwischen dem ausgetretenen Material und dem Nucleus pulposus besteht keine Verbindung mehr (Kluba und Roetman 2005c, S. 236). 
Die Behandlung erfolgt je nach Symptomatik und Schweregrad konservativ oder operativ (Niethard und Pfeil 2005, Koller 2011, Grimmer und Wurm 2011). Eine konservative Therapie wird primär bei Bandscheibenvorfällen ohne neurologische Ausfälle angestrebt. Bei Akutzuständen kann eine kurzzeitige Ruhigstellung (24 bis 48 Stunden) mittels einer Halskrause, kombiniert mit analgetischen oder muskelentspannenden Medikamenten schmerzlindernd wirken (Niethard und Pfeil 2005). Verschiedene Physiotherapietechniken und Wärmeanwendungen können Muskelverspannungen

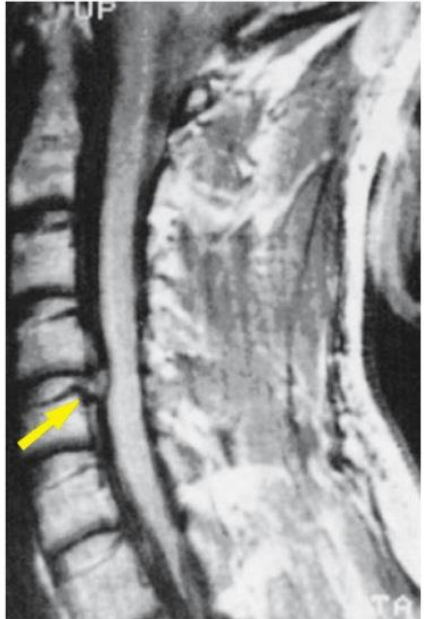

Abb. 1-12: MRT-Aufnahme eines Bandscheibenvorfalls zwischen dem 4. und 5 . Halswirbel (Thron 2006, S. 650)

lösen und somit zusätzlich hilfreich sein. Im Falle anhaltender Symptome besteht die Möglichkeit einer Röntgen- oder CT-gesteuerten Injektion von Lokalanästhetika (z.B. Bupivacain) und Cortison unmittelbar in die Nähe der schmerzauslösenden Veränderungen (Kluba und Roetman 2005a). Sollte auch diese therapeutische Maßnahme zu keiner dauerhaften Beschwerdebesserung führen, ist in den meisten Fällen nur ein operativer Eingriff hilfreich.

Gleichermaßen werden Bandscheibenvorfälle und Wirbelkanalstenosen, die mit neurologischen Ausfallerscheinungen (Myelopathie, Radikulopathie) einhergehen, primär operativ versorgt (Bohlmann et al. 1993, Emery et al. 1998, Koller 2011, Grimmer und Wurm 2011). In der Regel erfolgen die Eingriffe heute minimal invasiv über kleine Hautschnitte, wodurch das umliegende Gewebe möglichst geschont werden soll. Kleine Bandscheibenvorfälle und Einengungen des Spinalkanals behandelt man über kleine Zugänge im Nackenbereich (nach Krykholm), was als sogenannte „Schlüsselloch-Foraminotomie“ bezeichnet wird (Koller 2011). Hierbei werden der Wirbelkanal eröffnet, das vorgefallene Gewebe entfernt und anschließend die Wirbelkörper mit einer Titanplatte fixiert. Die eigentliche Bandscheibe bleibt bei dieser Art der Operation unberührt. Wesentlich häufiger wählt man im Bereich der HWS jedoch den Zugang von ventral (anteriore zervikale Diskektomie und Fusion; ACDF). Bei dieser Operationstechnik wird die Bandscheibe komplett entfernt und durch ein Kunststoffimplantat oder Knochenmaterial z.B. aus dem Beckenkamm ersetzt (Operation nach Cloward bzw. Smith-Robinson) (Smith und Robinson 1958, Bohlmann et al. 1993, Emery et al. 1998, Cherry 2002, Poeck und Hacke 2006, Buchowski et al. 2009, Koller 2011). Zusätzlich kann das betroffene Segment mit Hilfe einer Titanplatte fusioniert werden. Diese Maßnahmen führen zu einem Verlust der Beweglichkeit des betroffenen und möglicherweise zu degenerativen Veränderungen („Adjacent Segment Disease“) der angrenzenden Wirbelsegmente (Wigfield et al. 2002, Hilibrand und Robbins 2004, Goffin et al. 2004, Seo und Choi 2008, Buchowski 
et al. 2009). Mit der Implantation einer Bandscheibenprothese soll die Bewegung erhalten bleiben und die Degenration der angrenzenden Segmente verzögert bzw. verhindert werden (Galbusera et al. 2008a, Jaramillo-de la Torre et al. 2008, Bartels et al. 2008, Buchowski et al. 2009).

\subsection{Zervikale Bandscheibenprothesen}

\subsubsection{Historische Entwicklung}

Die Entwicklung der Bandscheibenprothesen lässt sich bis in die 1960er Jahre zurückverfolgen. Als erster ernsthafter Versuch der Bandscheibenarthroplastik wird eine von Fernstrom hergestellte Edelstahlkugel angesehen, die er 1956 erstmalig implantiert worden ist (Fernstrom 1966, Sekhon und Ball 2005, Fritsch und Pitzen 2006, Sethi et al. 2011). Erste Daten über die Implantation 75 dieser „Fernstrom-Prothesen“ wurden im Jahr 1964 publiziert (Reitz und Joubert 1964). Bis in die 70er Jahre implantierten auch andere Wirbelsäulenspezialisten derartige Kugelprothesen (McKenzie 1972). Jedoch führten potentielle Komplikationen der Implantation, welche sich in Form von Hypermobilität und Einsenkung der Metallkugel in die angrenzenden Wirbelkörper äußerten, zum raschen Verlassen des als Kugellager fungierenden Bandscheibenersatzes (Sekhon und Ball 2005, Fritsch und Pitzen 2006, Sethi et al. 2011) (Abb.1-13).

Durch Vergrößerung der Kontaktfläche zwischen Implantat und der Endplatte des Wirbelkörpers und einer damit einhergehenden, besseren Lastverteilung erschienen in den nachfolgenden Jahren zahlreiche neuartige Prothesentypen. Ende der 1980er Jahre entwickelte Cummins eine Bandscheiben-

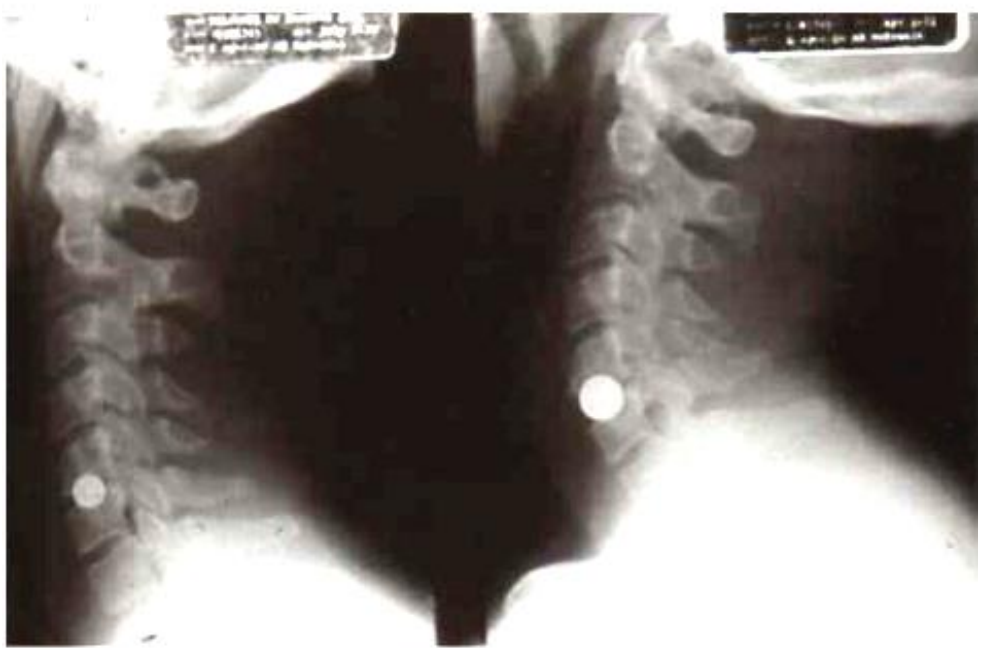
prothese nach dem „ball-andsocket-Design" (Fritsch und Pitzen

Abb.1-13: seitliche Röntgenaufnahme der HWS nach Implantation eines Kugelimplantates zwischen C5/C6. (McKenzie 2011, S. 8)

2006). Die aus Stahl gefertigte Prothese bestand aus einem oberen und einem unteren Anteil, die jeweils in die Wirbelkörper eingeschlagen wurden und nach dem Prinzip eines Kugelgelenkes funktionierte (Gelenkkugel im oberen Glied, Gelenkpfanne in der unteren Einheit). Sie legte den Grundstein für die Entwicklung der Prestige ${ }^{\circledR}$ LP Cervical Disc System (siehe 1.4.5.1) (Mummaneni et al. 2007, Traynelis 2011). 
2002 entwickelte Bryan ein neuartiges System zervikaler Bandscheibenprothesen, die anders als die bisherigen Metall-auf-Metall-Prothesen einen Polycarbonat-/ Polyurethankern beinhalten, der von zwei Metallschalen umschlossen wird (Bryan 2002). Dadurch sollte die hohe Reibung, die bei Prothesen mit Metall/Metall-Gelenkpaarung zu beobachten war, reduziert und der Materialabrieb verhindert werden. Ein Beispiel dieser Prothesenklasse stellt die Bryan ${ }^{\circledR}$ Cervical Disc dar (siehe 1.4.5.2). 2003 wurde schließlich die erste zervikale Bandscheibenprothese mit einer Materialkombination aus Kobalt-Chrom und ultrahochmolekularem Polyethylen entwickelt (McAfee et al. 2003), die sich bereits in der Hüft- und Kniegelenksarthroplastik bewährt hat (Fritsch und Pitzen 2006, Delamarter und Pradhan 2011). Ein Vertreter dieser Klasse ist die ProDisc-C ${ }^{\circledR}$ (Synthes, West Chester, Pennsylvania), die nach dem Prinzip des Kugelgelenkes aufgebaut ist. Dies ermöglicht eine Rotation entlang aller drei Achsen. Eine Translation ist bis auf wenige Millimeter in anterior/posterior-Richtung nicht möglich (Mehren und Mayer 2005, Delamarter und Pradhan 2011).

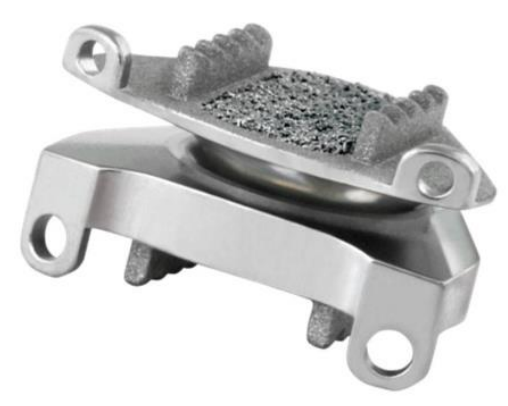

(A)

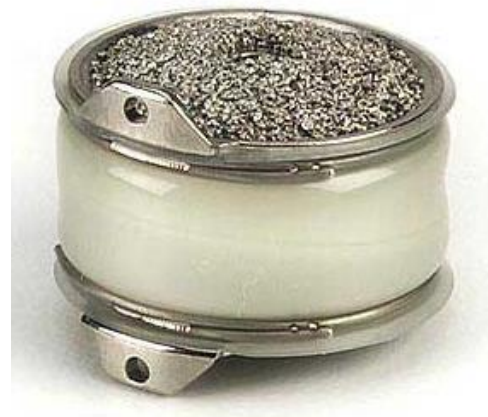

(B)

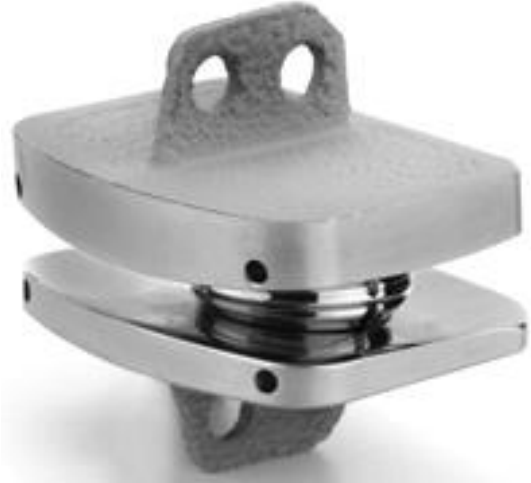

(C)

Abb. 1-14: Bandscheibenprothesen der Firma Medtronic Sofamor Danek: (A) Prestige ${ }^{\circledR}$ LP Cervical Disc, (B) Bryan ${ }^{\circledR}$ Cervical Disc System, (C) Maverick ${ }^{\mathrm{TM}}$ Disc. ("Used with the permission of Medtronic International Trading Sàrl @ 02/12/2010 Medtronic International Trading Sàrl”)

Allgemein ist zu erwähnen, dass die Arthroplastik der Wirbelsäule sehr lange einer ablehnenden Mehrheit gegenüberstand (McKenzie 2011). Als mögliche Gründe dafür werden persönliche und politische Überlegungen und nationales Ansehen diskutiert (Plato 1950). Ausschlaggebend wird jedoch die Einführung der bis heute etablierten Standardtherapie der anterioren zervikalen Diskektomie und Fusion (ACDF; siehe 1.3.2) bei chronischen Wirbelsäulenerkrankungen gewesen sein (Habela und McAfee 2011, McKenzie 2011). Diese wurde erstmals im Februar 1954 von Smith und Robinson durchgeführt (Smith und Robinson 1958) und galt als die Operationstechnik, die, einmal perfektioniert, alle Wirbelsäulenprobleme beheben sollte (McKenzie 2011). 


\subsubsection{Einteilung der Bandscheibenprothesen}

Mit der Entwicklung zahlreicher neuer Prothesentypen in den 1990er Jahren, brachte die "Cervical Spine Study Group" ein Klassifikationssystem zur Einteilung der zervikalen Bandscheibenprothesen heraus (Mummaneni und Haid 2004). Danach können die Prothesen anhand der Anzahl ihrer artikulierenden Gelenkflächen (nicht-, einfach oder zweifach artikulierend), dem Material der einzelnen Prothesenteile (Metall, Keramik, Polyethylen) und ihrem Design (austauschbare Bestandteile/modular; nicht-austauschbare Bestandteile/non-modular) unterschieden werden. Bestehen die artikulierenden Gelenkanteile aus verschiedenen Materialen, unterscheidet man weiter zwischen Prothesen mit Metall/Metall-, Metall/Polymer-, Keramik/Polymer- oder Keramik/KeramikGelenkpaarung (Sekhon und Ball 2005, Mummaneni et al. 2007, Galbusera et al. 2008a). Eine weitere Einteilung lässt sich anhand der biomechanischen Eigenschaften der Bandscheibenprothesen vornehmen. Nach dem Grad ihrer Führung und der daraus resultierenden Anzahl an Freiheitsgraden unterscheidet man starre und weniger starre ("bewegliche") Prothesen ("constrained"/"semiconstrained"/"unconstrained", siehe Abbildung 1-15) (Anderson und Rouleau 2004, Mummaneni und Haid 2004, Fritsch und Pitzen 2006, Galbusera et al. 2008a).

Die in Abbildung 1-15 abgebildete Variante einer "constrained“ Prothese stellt ein reines Kugelgelenk dar. Damit ist eine Rotation in alle drei Raumrichtungen möglich, während eine Translation nicht stattfindet. Demnach hat sie drei Freiheitsgrade. Sie weist ein festes Rotationszentrum auf (Galbusera et al. 2008a). Beispiel: Maveric ${ }^{\mathrm{TM}}$ Disc (Fa. Medtronic) (Abb. 1-14). Bandscheibenprothesen, die über das Gleitpaarungsprinzip verfügen („semi-constrained“) erlauben neben der Rotation um alle drei Achsen zusätzliche die Translation entlang einer Achse (Sekhon und Ball 2005, Fritsch und Pitzen 2006, Traynelis 2011). Ein Vertreter dieser Prothesen-Gruppe ist die Prestige $^{\circledast}$ LP (Fa. Medtronic), die vier Freiheitsgrade aufweist (Anderson und Rouleau 2004, Chang et al. 2007).

constrained

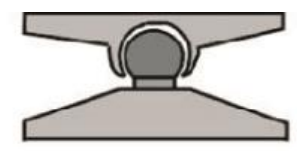

semi-constrained

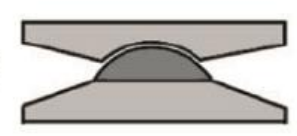

unconstrained
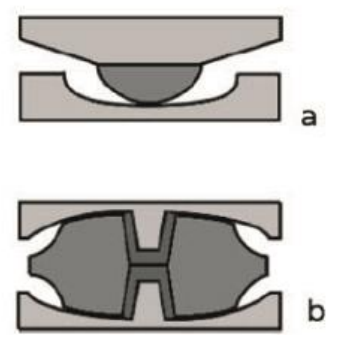

Abb. 1-15: Schematische Darstellung der ProthesenEinteilung anhand des Designs. Innerhalb der frei beweglichen Prothesen ("unconstrained") unterscheidet man Prothesen mit nicht-kongruenten, gleitenden Gelenkflächen (a), mit mehreren Gelenken (hier: zweifach-artikulierend) (b) und Prothesen mit verformbarem Kern (c). (Galbusera et al. 2008a, Fig. 1) 
Nichtgeführte Prothesen (z.B. Bryan ${ }^{\circledR}$ Cervical Disc; siehe 1.4.5.2) sind durch eine höhere Zahl an Freiheitsgraden gekennzeichnet und benötigen eine gute Weichteilspannung, damit eine angemessene Stabilität des Bewegungssegmentes nach der Implantation gewährleistet werden kann (McAfee et al. 2003). Sie weisen ein mobiles Rotationszentrum auf, wodurch leichte Ungenauigkeiten bei der Prothesenimplantation ausgeglichen werden können, andererseits jedoch eine stärkere Belastung der Facettengelenke entstehen kann (Phillips und Garfin 2005). Starrer geführte Prothesen mit weniger Freiheitsgraden und fixem Rotationszentrum schützen hingegen die Facette und weisen eine höhere Stabilität des Bewegungssegmentes auf, erleiden dadurch aber eine größere Beanspruchung im Knochen-Implantat-Interface (Phillips und Garfin 2005, Fritsch und Pitzen 2006).

\subsubsection{Indikationen und Kontraindikationen}

Grundsätzlich bestehen für die Implantation einer Bandscheibenprothese die gleichen Indikationen wie bei der Versorgung pathologischer HWS-Veränderungen (Spondylose, Facettenarthropathie, Nucleus-pulposus-Prolaps, isolierte degenerative Bandscheibenerkrankungen) mittels anteriorer Dekompression und Fusion (Fritsch und Pitzen 2006, Habela und McAfee 2011). Tabelle 1-1 gibt einen Überblick über Indikationen und Kontraindikationen.

\section{Indikation}

- 1- oder 2-Etagenpathologie mit Radikulopathie, Myelopathie oder Myeloradikulopathie

- Ausschöpfung konservativer Therapiemaßnahmen

- Osteochondrose mit ausreichend hohem Zwischenwirbelraum

- $\quad$ Patientenalter zwischen 30. und 50. Lebensjahr

\section{Kontraindikation}

- $\quad$ Alter <18 Jahren und >60 Jahren

- (zervikale) Infektionen

- $\quad$ Systemische Erkrankungen (AIDS, HIV, Hepatitis)

- Allergien gegen Prothesenmaterial

- Schwangerschaft

- Rheumatoide Arthritis/Spondylitis ankylosans, Autoimmunerkrankungen

- Vorangegangene Frakturen, Tumoren

- metabolische Störungen, krankhafte Fettsucht (BMI >40), Diabetes mellitus

- Osteoporose, Facettenarthrose, Spondylolisthese

- Instabilität des Bewegungssegmentes

- Den Spinalkanal einengende Erkrankungen (OPLL)

- $\quad$ Z.n. zervikaler Laminektomie

Tab. 1-1: Indikationen und Kontraindikationen für die Implantation zervikaler Bandscheibenprothesen (Fritsch und Pitzen 2006, Habela und McAfee 2011). 


\subsubsection{Anforderungen an eine Bandscheibenprothese}

Unabhängig von der Art und dem Design werden zahlreiche Anforderungen an eine Bandscheibenprothese gestellt. Dazu gehört in erster Linie der Erhalt oder die Wiederherstellung von Beweglichkeit, Stabilität und Komprimierbarkeit des Wirbelsäulensegmentes, in welches die Prothese implantiert wird (McKenzie 2011). Im Gegensatz zur anterioren Dekompression und Fusion, die durch den Bewegungsverlust über einen längeren Zeitraum zu pathologischen Veränderungen der angrenzenden Bewegungssegmente („Adjacent Segment Disease“) führt (Wigfield et al. 2002, Goffin et al. 2004, Seo und Choi 2008, Buchowski et al. 2009), soll so die Degeneration benachbarter Wirbelsäulensegmente reduziert bzw. verhindert werden (Galbusera et al. 2008a, Jaramillo-de la Torre et al. 2008, Bartels et al. 2008, Buchowski et al. 2009). Dabei sollten die physiologischen Abläufe, sowie die Lastverteilung möglichst unbeeinträchtigt bleiben und ein natürliches Bewegungsausmaß gewährleistet werden (Sekhon und Ball 2005, Phillips und Garfin 2005, Galbusera et al. 2008a). Gleichzeitig sollte die Prothese über eine hohe Haltbarkeit und Abriebfestigkeit verfügen. Die Reibung der artikulierenden Gelenkflächen sollte daher so gering wie möglich sein, um einen Verschließ der Komponenten zu vermeiden (Phillips und Garfin 2005). Für eine optimale Kraftübertragung und zur Minimierung des Druckes sind außerdem eine individuelle Abmessung des Bandscheibenfaches, sowie die Wahl der entsprechenden Prothesengröße unerlässlich (White und Panjabi 1990, Fritsch und Pitzen 2006). Die Bandscheibenprothesen müssen daher in unterschiedlichen Größen und in möglichst kleinen Abstufungen verfügbar sein. Eine Lockerung der implantierten Bandscheibenprothese sollte ebenfalls ausbleiben (Phillips und Garfin 2005).

\subsubsection{Prothesen}

Bei den in dieser Studie untersuchten Komponenten handelte es sich um die Bandscheibenprothesen Prestige ${ }^{\circledR}$ LP Cervical Disc System (Medtronic Sofamor Danek, Memphis, TN) und Bryan ${ }^{\circledR}$ Cervical Disc System (Medtronic Sofamor Danek, Memphis, TN).

\subsubsection{Prestige ${ }^{\circledast}$ LP Cervical Disc System}

Bei der Prestige ${ }^{\circledR}$ LP Cervical Disc (Markteinführung: 2004) handelt es sich um die Weiterentwicklung der von Brian Cummins Ende der 80er Jahre entwickelten Cummins-Prothese, die erstmals 1991 in Bristol (UK) implantiert wurde (Sekhon und Ball 2005, Bartels et al. 2008, Traynelis 2011, Medtronic 2013). Nach der Prestige ${ }^{\circledR}$ I (1998), Prestige ${ }^{\circledR}$ II (1999), Prestige ${ }^{\circledR}$ ST (2002) und Prestige ${ }^{\circledR}$ STLP (2003) stellt sie die fünfte Generation einer der ältesten Bandscheibenprothesen dar (Mehren und Mayer 2005, Mummaneni et al. 2007). Die Zulassung der FDA (U.S. Food and Drug Administration 2009) erhielt die Prestige ${ }^{\circledR}$ Cervical Disc System am 16. Juli 2007. 
Es handelt sich bei dieser Prothese um ein Titan-Keramik-Gemisch, bestehend aus einem oberen und einem unteren Element, welche über ein Kugelgelenk artikulieren (Abb. 1-16). Die Prothese ist so konstruiert, dass die Gelenkkugel im oberen Glied, die Gelenkpfanne in der unteren Einheit eingearbeitet ist („,ball-and-socket-Design“).

Diese Konstruktion ermöglicht eine uneingeschränkte ("unconstrained") Rotation entlang der drei Raumachsen (Bartels et al. 2008). Die Pfanne erlaubt durch eine größere

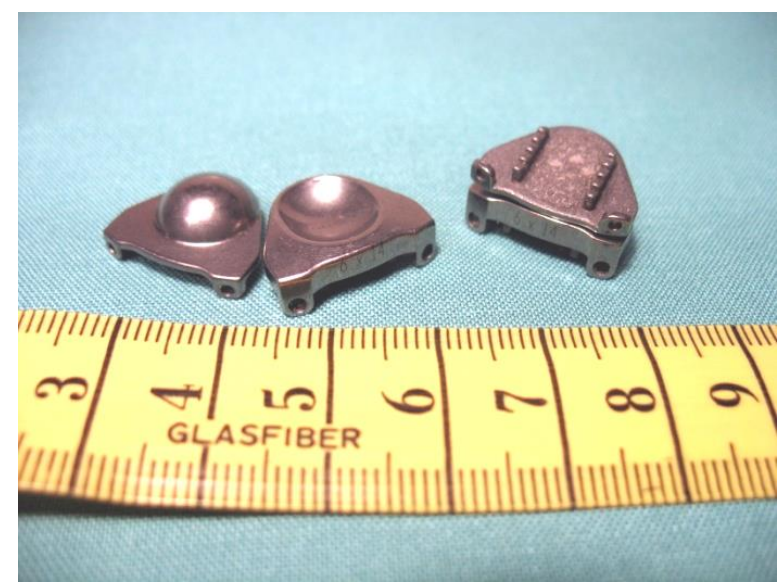

Abb. 1-16: Prestige ${ }^{\circledR}$ LP Cervical Disc System; links: oberer Teil mit Gelenkkugel, unterer Teil mit Gelenkpfanne; rechts: zusammengesetzte Prothese, Blick auf raue Oberfläche und Verzahnungsleisten

Ausdehnung in der Sagittalen lediglich eine Translation in diese Richtung ("semi-constrained")

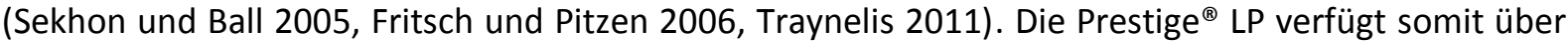
vier Freiheitsgrade. Mittels spezieller Verzahnungsleisten an Ober- und Unterseite wird die Prestige ${ }^{\circledR}$ LP Disc in den Wirbelkörpern befestigt (Mummaneni et al. 2007, Traynelis 2011). Zudem ist die raue Oberfläche mit einem Plasmaspray beschichtet, welches das Einwachsen des Knochens fördert und zusätzliche Haftung verschafft (Sekhon und Ball 2005, Mummaneni et al. 2007). Durch die Verfügbarkeit unterschiedlicher Prothesengrößen kann die Prothese individuell angepasst werden. In der Literatur lassen sich nur sehr wenige Daten über die Anzahl der Implantationen der Prestige ${ }^{\circledR}$ finden. Sekhon und Ball berichteten 2005 über insgesamt 500 implantierte Modelle (Stand: Dezember 2004) (Sekhon und Ball 2005).

\subsubsection{Bryan $^{\circledR}$ Cervical Disc System}

Bei der Bryan ${ }^{\circledR}$ Disc handelt es sich um einen einteiligen, aus mehreren Materialien gefertigten, Bandscheibenersatz (Mehren und Mayer 2005, Sasso und Martin 2011a). Sie wurde erstmals im Jahr 2000 in Europa und 2002 in den USA implantiert (Sasso et al. 2007). Seitdem wurden weltweit über 20000 Implantate verkauft (Medtronic 2012). Am 12. Mai 2009 erhielt die Bryan ${ }^{\circledR}$ Cervical Disc die Zulassung der FDA (U.S. Food and Drug Administration 2012). Zwei konvexe Titanplatten bilden die Grund- und Deckplatte und werden am Wirbel fixiert (Abb. 1-17 und 1-18). Die poröse Oberfläche ermöglicht zusätzlich das Einwachsen des Knochens und gewährleistet somit den festen Halt im Bandscheibenfach (Goffin et al. 2002, Jensen et al. 2005, Mehren und Mayer 2005). 
Die Beweglichkeit der Prothese basiert auf einem flexiblen, bi-artikulierenden PolycarbonatPolyurethan- Kunststoffkern (Anderson und Rouleau 2004, Bartels et al. 2008, Sasso und Martin 2011a), welcher von einer elastischen Polyurethan-Hülle umschlossen und geschützt wird (Abb. 1-18). Innerhalb dieser Hülle dient injizierte Kochsalzlösung als Schmiermittel und sorgt für einen dämpfenden Effekt bei axialer Belastung (Sasso und Martin 2011a). Die Bryan $^{\circledR}$ Disc ist eine nichtgeführte ("unconstrained") Bandscheibenprothese (Anderson und Rouleau 2004, Galbusera et al. 2008a). Sie besitzt sechs Freiheitsgrade (Rotation um die drei Raumachsen: Flexion-Extension, Lateralflexion und Axialrotation, sowie Translation in sagittaler und seitlicher Richtung und axiale Stauchungsfähigkeit) (Mehren und Mayer 2005) und gleicht darin der natürlichen Beweglichkeit der Bandscheibe (Patwardhan et al. 2010). Die Verfügbarkeit

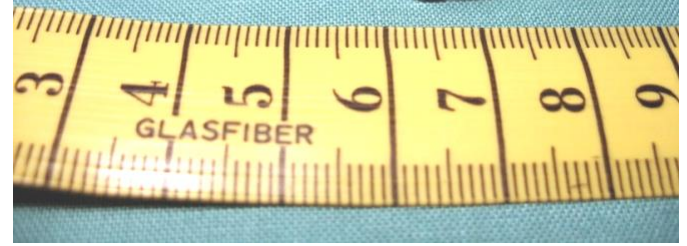

Abb. 1-17: Bryan ${ }^{\circledR}$ Cervical Disc System; Die Polyurethanhülle umschließt den Kunststoffkern und verbindet die beiden Deckplatten mit poröser Oberfläche.

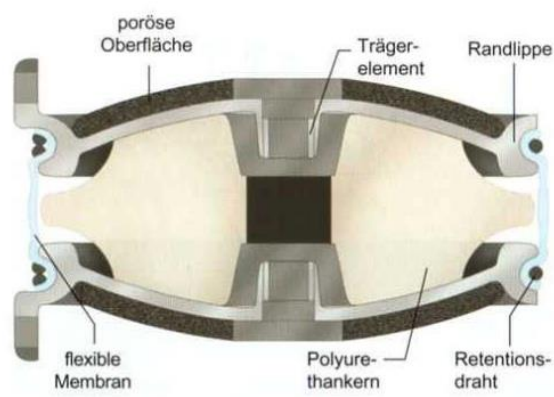

Abb. 1-18: Bryan® Cervical Disc System: Querschnitt (Sasso und Martin 2011b, S. 113)

unterschiedlicher Größen ermöglicht eine individuelle Anpassung an die Verhältnisse des Bandscheibenfaches.

\subsection{Biomechanik}

\subsubsection{Bestimmung der Bewegungsstruktur}

Um die genaue Lage eines Körpers im Raum zu bestimmen, benötigt man die Koordinaten von 3 auf ihm liegenden Punkten, die einen festen Abstand zueinander haben und nicht auf einer Linie liegen (Nägerl et al. 1995, Kettler et al. 2004, Haberl et al. 2004). Verschiebt man einen Körper, resultiert daraus eine Änderung der Koordinaten der festgelegten Punkte und ermöglicht so den Rückschluss auf die abgelaufene Bewegung. Dabei differenziert man zwischen zwei Bewegungsarten: Translations- und Rotationsbewegung. Die Verschiebung eines Körpers entlang einer Achse (Translation) ist in alle drei Raumrichtungen möglich und wird als Versatz s (entlang einer Achse) bezeichnet. Eine Positionsänderung durch Drehbewegungen (Drehwinkel $\alpha$ ) kann sich gleichermaßen um die drei Raumachsen abspielen. Demzufolge besitzt ein Gegenstand im Raum sechs Freiheitsgrade. 

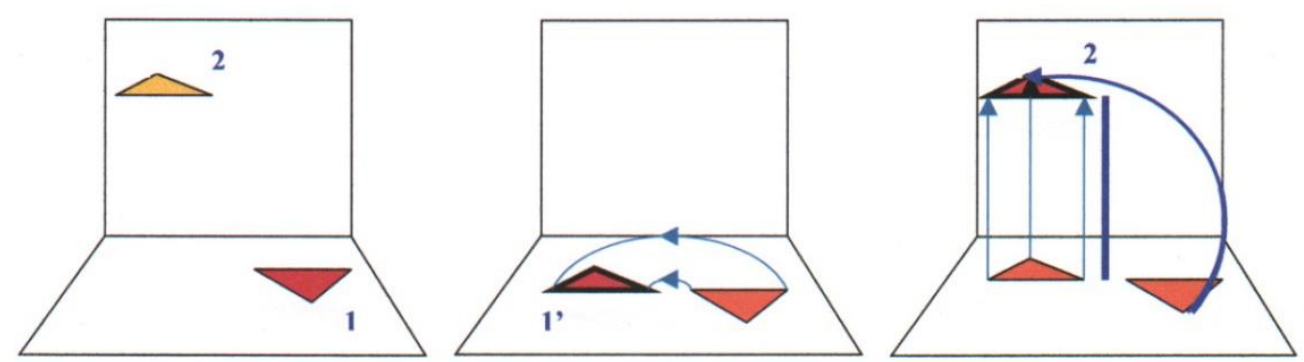

Abb. 1-19: Bewegung eines Körpers im dreidimensionalen Raum; Die Bewegung des Körpers von Position 1 nach 2 kann in zwei Teilbewegungen aufgeschlüsselt werden: 1 . in eine Rotation von Position $1 \mathrm{zu} 1^{\text {' }}$ und 2. in eine Translation entlang der Achse zur Position 2. (Bockermann 2004, S. 20)

Nach dem Satz von Chasles lässt sich jede Bewegung durch eine Verschraubung beschreiben (Teichmann 1973). Die kürzeste Verbindung zwischen der Ausgangs- und Endposition lässt sich demnach mit einer Bewegung beschreiben, welche sich wieder in eine Drehung um den Winkel $\alpha$ um eine Achse und die Translation entlang derselben Ache aufteilt. Der Quotient aus den Beträgen des Versatz $s$ und des Drehwinkels $\alpha$ entspricht der Schraubsteigung $\zeta(\zeta=|s| /|\alpha|)$. Zwischen beiden Positionen kann der Körper theoretisch jede denkbare Zwischenposition einnehmen, wenn die Lageänderung nicht differentiell klein ist (Wolf 1947). Im Falle der Erfassung von differenziell kleinen Änderungen, kann davon ausgegangen werden, dass die tatsächliche Bewegung erfasst wird. Für die differenzielle Schraubachse ergibt sich folglich $\zeta(\alpha)=\mathrm{d} s / \mathrm{d} \alpha$.

Eine feste Schraubachse liegt während einer Bewegung nur sehr selten vor (Nägerl et al. 1992, Kettler et al. 2004). Vielmehr ändert Sie ihre Position. Die Schnittpunkte der momentanen Schraubachse mit einer definierten Ebene ergeben die Rastpolkurve, mit deren Hilfe die Achswanderung im Rahmen einer Bewegung visualisiert werden kann.

\subsubsection{Grundlagen der Biomechanik}

Auf die Bewegungssegmente der Wirbelsäule wirken täglich zahlreiche Kräfte ein, die zu einer permanenten Veränderung des Bewegungszustandes führen. In diesem Zusammenhang spielen Kräfte, welche durch die Kontraktionswirkung der am Segment ansetzenden Muskeln entstehen, die größte Rolle. Im Bereich der Halswirbelsäule gehen zusätzliche Kraftwirkungen von der Gewichtskraft des Kopfes und diversen anderen Faktoren (Bremsen und Beschleunigen, Gewichte von Schutzhelmen, etc.) aus (Friedrich und Lühmann 2005).

Grundsätzlich lässt sich die Gesamtheit der auf einen Körper einwirkenden Kräfte (= Kraftsystem) auf zwei Komponenten vereinfachen: eine resultierende $\mathbf{K r a f t} \underline{\underline{F}}_{R}$, welche aus der vektoriellen Summe aller angreifenden Kräfte bestimmt werden kann und ein kräftefreies Drehmoment I (Assmann und Selke 2006). Verläuft die Wirkungslinie von $F_{R}$, auf welcher die resultierende Kraft frei verschoben werden kann, durch den Schwerpunkt eines Körpers, resultiert eine reine Translation (Fließbach 2007). In diesem Fall ist T gleich null. Eine außerhalb des Schwerpunktes angreifende Kraft erzeugt hingegen ein Drehmoment (Fercher 1999). Es errechnet sich aus der Kraft F, dem Abstand L dieser 
Kraft vom Schwerpunkt des Körpers (Hebel) und steht senkrecht auf ihnen ( $T=L \times F)$. Die Wirkung des Drehmomentes auf einen Körper ist unabhängig vom Angriffsort immer gleich, weshalb es auch als freier Vektor bezeichnet werden kann. Es ist unerheblich, ob Drehmoment und Drehachse dabei dieselbe Richtung haben. Eine an einem beliebigen Punkt angreifende Kraft kann durch die Addition der Kraft "0" (Kraft und Gegenkraft gleichen Betrages) am Schwerpunkt in eine dort angreifende Kraft und ein reines Drehmoment umgeformt werden (Nägerl 1990). Es lassen sich unendlich viele Variationen aus $L$ und $\mathrm{F}$ erzeugen, die ein und dasselbe Drehmoment beschreiben, solange deren Vektoren das gleiche Produkt haben und in parallelen Ebenen liegen. Analog zur resultierenden Kraft $F_{R}$ lassen sich Drehmomente, die um die drei Raumachsen wirken,

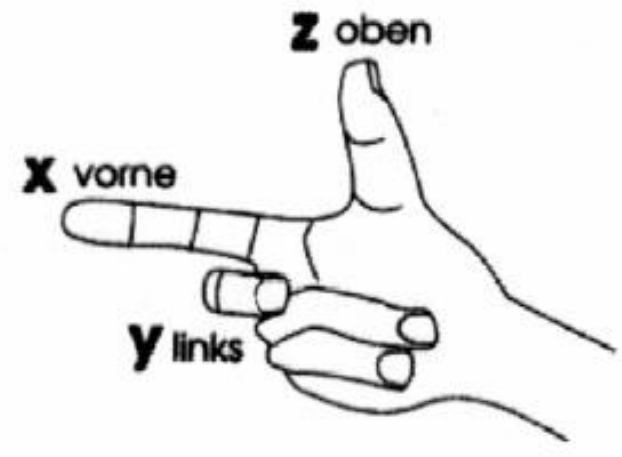

Abb. 1-20: Rechte-Hand-Regel zur Bestimmung der Richtung eines Drehmomentes. Daumen= Hebel, Zeigefinger= Kraft(richtung), Mittelfinger $=$ Richtung des Drehmomentes. (modifiziert nach Krismer 1996, S. 16)

in ein resultierendes Drehmoment $T_{R}$ umformen. Verlaufen $F_{R}$ und $T_{R}$ parallel, kann die dadurch entstehende Einwirkung auf einen Körper durch zwei verschiedene Bezugssysteme (Kraft bzw. Drehmoment) beschrieben werden (=äquivalentes Kraftsystem) (Wolf 1931, Nägerl 1990).

\section{$\underline{\text { Kraftschraube }}$}

Wird ein Kraftsystem auf eine resultierende $K_{r a f t} F_{R}$ und ein dazu paralleles Drehmoment $T_{R}$ reduziert, spricht man von einer Kraftschraube (Mansour 2001). Sie ist eindeutig definiert durch den Fußpunkt des Kraftvektors in einer definierten Ebene und einen normierten Richtungsvektor (jeweils zwei Freiheitsgrade), sowie den Betrag des zur Kraft parallel gerichteten Drehmomentes $T$ und den Betrag der resultierenden $\mathrm{Kraft}_{\mathrm{R}}$ mit jeweils einem Freiheitsgrad. Übertragen auf die Natur kann das Zusammenspiel verschiedener Muskeln biophysikalisch durch eine Kraftschraube beschrieben werden (Mansour 2001). In dieser Studie wurde die einfachste Form einer Kraftschraube gewählt, um die biomechanischen Eigenschaften der Präparate zu untersuchen. Dazu erfolgte die Applikation einer axialen Vorlast $F_{Z}\left(0,0, F_{Z}\right)$ und eines parallelen Drehmoments $T_{Z}\left(0,0, T_{Z}\right)$.

\subsubsection{Viskoelastizität}

Die Belastung der Wirbelsäule führt aufgrund der elastischen Eigenschaften zu einer Dehnung von Bändern und Bandscheiben (siehe 1.2.1.3 und 1.2.1.4) (Nägerl 1990). Dadurch können abrupte Bewegungen oder Richtungswechsel in einem bestimmten Ausmaß gedämpft werden. Die Auslenkung $s$ ist dabei der einwirkenden Kraft $\mathrm{F}$ annähernd proportional. Bei einer Entlastung stellt sich die Gesamt-Dehnung nach unbestimmter Zeit wieder auf den festen (plastischen) Anteil zurück. 
Die (visko-)elastische Dehnung ist demnach vollständig reversibel. Die Anlage einer sich sinusartig ändernden Kraft bewirkt somit eine dem ursächlichen Erreger ähnliche Bewegung der mobilen Struktur (Wirbelkörper), die jedoch zeitlich versetzt (phasenverschoben) ist. Diese Gegebenheit bezeichnet man als Hysterese.

\subsubsection{Widerstandszentrum}

Das Widerstandszentrum stellt eine wichtige Kenngröße biophysiologischer Systeme dar. Dieser Punkt wird dadurch charakterisiert, dass das Anlegen einer Kraft an den Körper eine reine Translation in Kraftrichtung bewirkt. Eine zusätzliche rotatorische Bewegungskomponente besteht nicht. Greift hingegen ein reines, kräftefreies Drehmoment an das System an, resultiert eine Rotation um diesen Punkt (Nägerl 1990). Auf ein Wirbelsegment übertragen, bei dem der untere Wirbel ortsfest fixiert ist, lässt sich das Widerstandzentrum als Durchstoßpunkt der z-Achse durch eine Ebene auf Höhe des Übergangs der Bandscheibe zum oberen Wirbelkörper bestimmen (Nägerl 1990). Senkrecht dazu wird der Punkt von der $\mathrm{x}$-Achse (sagittale Richtung) und der $\mathrm{y}$-Achse (transversale Richtung) getroffen.

Die Änderung $\Delta z$ durch das Anlegen einer axialen Kraft $F_{z}$ führt zu einer Dehnung bzw. Stauchung der Bandscheibe. Nach dem Hookeschen Gesetz gilt $F_{Z}=E \cdot \Delta z \cdot(A / h) \cdot \Delta z$. Dabei bezeichnet $E$ den Elastizitätsmodul, A die Fläche und h die Höhe der Bandscheibe (Mansour 2001).

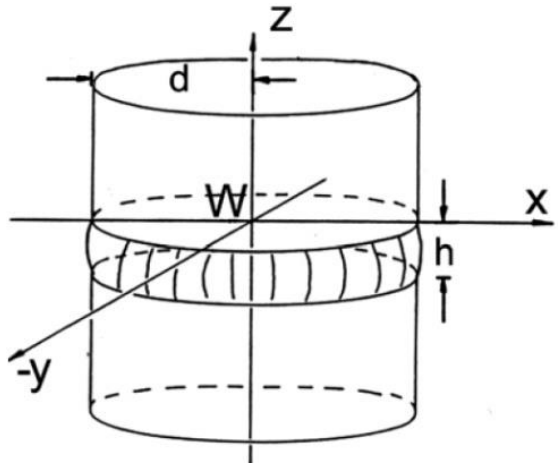

Abb. 1-21: Schema einer Synarthrose; Das Widerstandszentrum $\mathrm{W}$ liegt im Ursprung des Koordinatensystems. $\mathrm{h}=$ Höhe und $\mathrm{d}=$ Radius der elastischen Scheibe (Mansour 2001, S. 30)

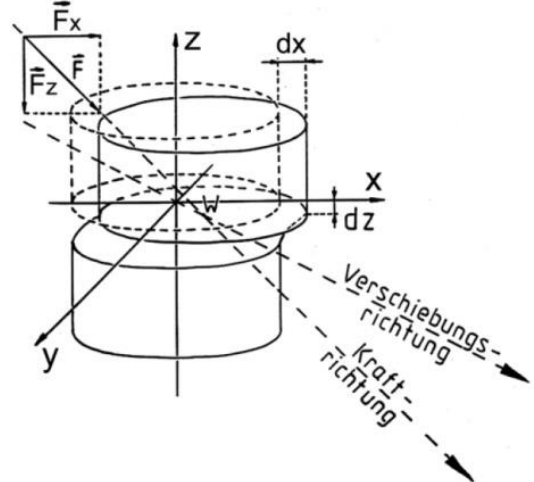

Abb. 1-22: Auswirkung einer $\operatorname{Kraft} F\left(F_{x}, 0, F_{z}\right)$ auf eine Synarthrose. Infolge des höheren Elastizitätsmoduls ist der vertikale Verschiebevektor kleiner, als der horizontale Verschiebevektor. In der Folge ist die Stauchung kleiner als die Abscherung (Kraftrichtung $\neq$ Verschiebungsrichtung). (Mansour 2001, S. 32)

Wirkt stattdessen eine Kraft $F_{X}$ bzw. $F_{Y}$ auf das System, kommt es zu einer Abscherung der Zwischenwirbelscheibe um $\Delta x$ bzw. $\Delta y$. Es gilt $F_{x}=G \cdot(A / h) \cdot \Delta x$ bzw. $F_{y}=G \cdot(A / h) \cdot \Delta y(G=S c h u b-b z w$. Schermodul) (Fercher 1999, Mansour 2001).

Der Elastizitätsmodul E und Schermodul G sind unterschiedlich groß (Nägerl 1990). Dies ist dadurch zu erklären, dass die entgegengesetzte Kraft eines Körpers auf Scherung und Stauchung verschieden ist. Legt man eine Kraft in Richtung der Winkelhalbierenden zwischen $x$ - oder $y$-Achse und z-Achse 
an, weicht die resultierende Bewegung von der ursprünglichen Kraftlinie ab. Der Elastizitätsmodul ist doppelt bis dreifach so groß, wie der Schermodul, weshalb die Bandscheibe für Stauchung und Dehnung steifer ist, als für die Abscherung. Der Umfang der horizontalen Translation ist folglich höher als die in vertikaler Richtung und der Verschiebevektor flacher als der Kraftvektor.

\subsubsection{Biomechanische Eigenschaften intakter Segmente}

Die Beweglichkeit der Wirbelsäule ist von zahlreichen Faktoren abhängig, die einen entscheidenden Einfluss auf die Bewegungsstruktur nehmen. Mehrere Studien an „Junghanns'schen Segmenten“ konnten zeigen, dass die Bewegungseigenschaften der Wirbelsäule einerseits auf dem Betrag und der Position der axialen Vorlast beruhen, andererseits auf dem Ausmaß des Rotationswinkels $\alpha$ und der geometrischen Anordnung der führenden Gelenke (Wachowski et al. 2009a; Wachowski et al. 2010). Dabei spielen Bandapparat und Bandscheibe (siehe 1.2.1.3 bzw. 1.2.1.4) eine wichtige Rolle bei der Regulation der Bewegungsquantität. Mit steigendem Rotationswinkel kommt es zu einer Kompression/Dehnung und Abscherung der Bandscheibe. Die reflektorisch unter Spannung stehenden Bänder, sowie die resultierenden Gegenkräfte durch Stauchung und Scherung vermindern sukzessiv den Bewegungsspielraum. Die Steifigkeit des Segmentes steigt an. Dadurch wird eine übermäßige Auslenkung, die zu einer möglichen Schädigung der Wirbelsäule führen kann, verhindert (Nägerl 1990).

Treten während eines Bewegungsablaufes Gelenkflächen in Kontakt, wird die Bewegung fortan durch die entstandenen Kontaktpunkte geführt und aufgrund dessen die Bewegungsqualität maßgeblich definiert (Nägerl et al. 1995, Kalscheuer 2001, Mansour et al. 2004, Wachowski 2005). Zeitgleich geht dies mit einer Einschränkung der Freiheitsgrade einher und resultiert ebenfalls in einem Anstieg der Steifigkeit. Neben den beiden Facettengelenken verfügen die Segmente der Halswirbelsäule weiterhin über zwei Uncovertebralgelenke. Im Vergleich zu technischen Kugelgelenken, die lediglich drei Freiheitsgrade aufweisen (Rotation), verfügen die biologischen Kugelgelenke über fünf Freiheitsgrade (Nägerl 1990). Die Inkongruenz der Gelenkflächen ermöglicht neben den drei Rotationsrichtungen zusätzlich die Bewegung in zwei Translationsrichtungen. Die vier Gelenke des Halswirbelsegmentes wirken in einem System zusammen, weshalb im Rahmen einer Bewegung mehrere Gelenke auf einmal kraftschlüssig sein können. Generell ist davon auszugehen, dass die Gelenkkräfte der vier beteiligten Gelenke windschief zueinander liegen und sich nicht schneiden. Dadurch kommt es beim Kraftschluss zu einer Versteifung des Systems, da es sich um eine Drehung um sich nicht schneidende Achsen handelt (Wachowski et al. 2007). Artikulieren zwei Gelenke zeitgleich, liegen daher nur vier Freiheitsgrade, bei drei artikulierenden Gelenken nur drei und bei Kraftschluss aller vier Gelenke lediglich zwei Freiheitsgrade vor. Da die letzte Konstellation einer Blockierung des Segmentes entsprechen würde, ist zu vermuten, dass in der Mehrzahl der 
physiologischen Situationen nicht alle Gelenke gleichzeitig kraftschlüssig sein können. Je nach Vorbelastung und Funktion kann das Segment demnach zwei (Kraftschluss aller Gelenke) bis sechs (keinerlei Kraftschluss der Gelenke) Freiheitsgrade aufweisen (Nägerl 1990, Nägerl et al. 1995).

Axial auf die Wirbelsäule einwirkende Kräfte (z.B. Kontraktionswirkung der ansetzenden Muskulatur) können je nach Betrag und Angriffspunkt so entscheidende Bedeutung für die Beweglichkeit des Segmentes sein (Wachowski et al. 2009b). Mit zunehmender Kompression der Wirbelsegmente kann es zu einer Änderung der Druckbelastung der Facettengelenke kommen (El-Bohy et al. 1989), einhergehend mit abweichenden Führungsbedingungen und einem modifizierten Verhalten des Systems.

Eine ventral des Widerstandszentrums angebrachte Vorlast resultiert in einer Flexionsstellung der benachbarten Wirbel und kann im Extremfall die Entkopplung der Wirbelbogengelenke herbeiführen. Dieser Mechanismus könnte dazu führen, dass eine Gelenkführung lediglich im Bereich größerer Rotationwinkel in Kraft tritt, bzw. vollständig aufgehoben wird (Nägerl et al. 1995, Mansour et al. 2004, Wachowski et al. 2009a, Wachowski et al. 2009b, Wachowski et al. 2010). Die ursprünglichen Freiheitsgrade bleiben somit (länger) erhalten und korrelieren mit einer entsprechend gesteigerten Beweglichkeit. Analog ist ein Abfall der Segmentsteifigkeit zu beobachten. In den Studien von Wachowski et al. konnte zudem eine Abhängigkeit des Migrationsverhaltens der momentanen Schraubachse (IHA) von Flexions- bzw. Extensionsstatus und Vorlastbetrag beschrieben werden (Wachowski et al. 2009b). Im Vergleich zu einer zentral im Widerstandszentrum angreifenden Kraft zeigte sich unter ventraler Vorlast eine vermehrte Wanderung, bei dorsal gelegener Vorlast war hingegen eine Abnahme der Achswanderung zu verzeichnen. Dabei kam es mit Eintritt der alternierenden Gelenkführung zu einer Achsenmigration in Richtung des artikulierenden Gelenkes. Die Ausrichtung der Achsen blieb trotz variierender Vorlastkonstellationen nahezu konstant und verlief nicht parallel zum anliegenden Drehmoment.

Daraus lässt sich schlussfolgern, dass die Gelenke eine entscheidende Rolle bei der Ausrichtung und Lokalisation der momentanen Schraubachse des intakten Segmentes spielen (Wachowski et al. 2007). In vivo können kleine Veränderungen der Muskelaktivität (Vorlastverschiebung) neue Systemmerkmale initiieren und infolge neuer Kontaktpunkte bzw. Gelenkführung die Position der momentanen Schraubachse modifizieren.

\section{Fragestellung}

In der vorliegenden Studie soll die Frage beantwortet werden, wie die Implantation zweier verschiedener Arten von Bandscheibenprothesen das Bewegungssystem des C3/C4-Segmentes beeinflusst. 


\section{Material und Methoden}

\subsection{Messprinzip}

Die Zielsetzung dieser Studie lag in der Analyse des Einflusses der Bandscheibenprothesen Prestige ${ }^{\circledR}$ LP Cervical Disc System (Medtronic Sofamor Danek, Memphis, TN) und Bryan ${ }^{\circledast}$ Cervical Disc System (Medtronic Sofamor Danek, Memphis, TN) auf die Kinematik eines Halswirbelsäulen-Segmentes. Untersucht wurde das „Junghanns'sche Segment" (Junghanns 1977) C3/C4 von acht menschlichen Halswirbelsäulen. Die grundlegende Voraussetzung lag zunächst in der genauen Ermittlung der physiologischen Eigenschaften des intakten Segmentes. Dies erforderte die hochauflösende Aufnahme und Analyse der Bewegungsstruktur der Versuchsobjekte.

Dazu wurde zu Beginn eine starre Verbindung des C4-Wirbels mit der Messapparatur geschaffen. Anschließend erfolgte die Applikation eines zyklischen Drehmomentes an den jeweiligen C3-Körper in axialer $\left(T_{z^{-}}\right)$, sagittaler $\left(T_{x^{-}}\right)$und frontaler Ausrichtung ( $T_{y^{-}}$-Richtung). Gleichzeitig wurden verschiedene Vorlastgrößen in unterschiedlichen Positionen angelegt. Die resultierende Bewegungsänderung wurde sechsdimensional, differentiell klein aufgenommen und anhand der ermittelten Daten deren Struktur analysiert und rekonstruiert. Dadurch war es wiederum möglich, die jeweilige Positionsveränderung als momentane Schraubbewegung zu beschreiben und den tatsächlichen Bewegungsvorgang zu erfassen.

Im Anschluss an die exakte Bestimmung der physiologischen Charakteristika folgte die Resektion der Bandscheibe und deren Ersatz mittels Prestige ${ }^{\circledR}$ LP Cervical Disc System sowie Bryan ${ }^{\circledR}$ Cervical Disc System. Alle übrigen experimentellen Variablen blieben unverändert.

\subsection{Material und Morphologie}

\subsubsection{Halswirbelpräparate}

Die verwendeten Halswirbelsegmente entstammen Autopsiematerialien des anatomischen Institutes der Ernst-Moritz-Arndt-Universität Greifwald. Dabei handelte es sich um sechs weibliche und zwei männliche Halswirbelsäulen (Durchschnittsalter: 69,75 Jahre \pm 13,95 Jahre; siehe Tab. 2.1). Diese wurden in Kooperation mit Herrn Prof. Fanghänel zur Verfügung gestellt. Es lag ein positives Votum der Ethikkommission der medizinischen Fakultät der Universität Göttingen vor (Antrags-Nummer: $17 / 12 / 09)$. 
Zunächst fand eine konventionell-radiologische und eine computertomographische Untersuchung statt, welche dem Ausschluss von pathologischen Veränderungen, der en-bloc entnommenen Wirbelsäulen (C1 bis Th1 bzw. C1 bis S5), diente (Abb. 2-1 und Abb. 2-2). Als Ausschlusskriterium galt das Vorliegen von Fakturen und/oder signifikante degenerative Veränderungen des Discus intervertebralis und der Artt. zygapophysiales.

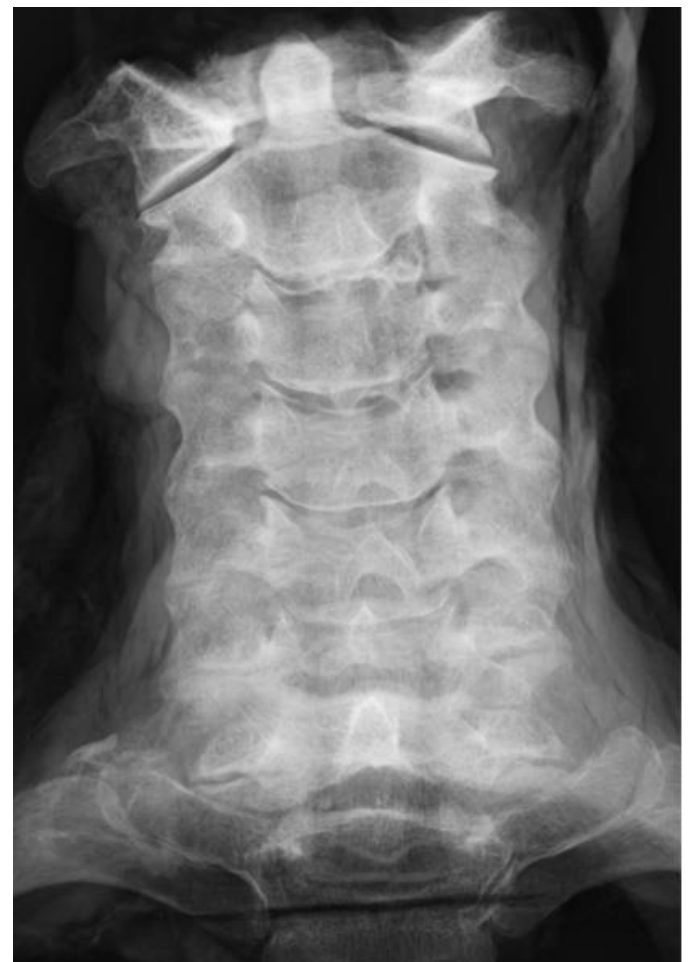

Abb. 2-1: anterior-posterior Aufnahme

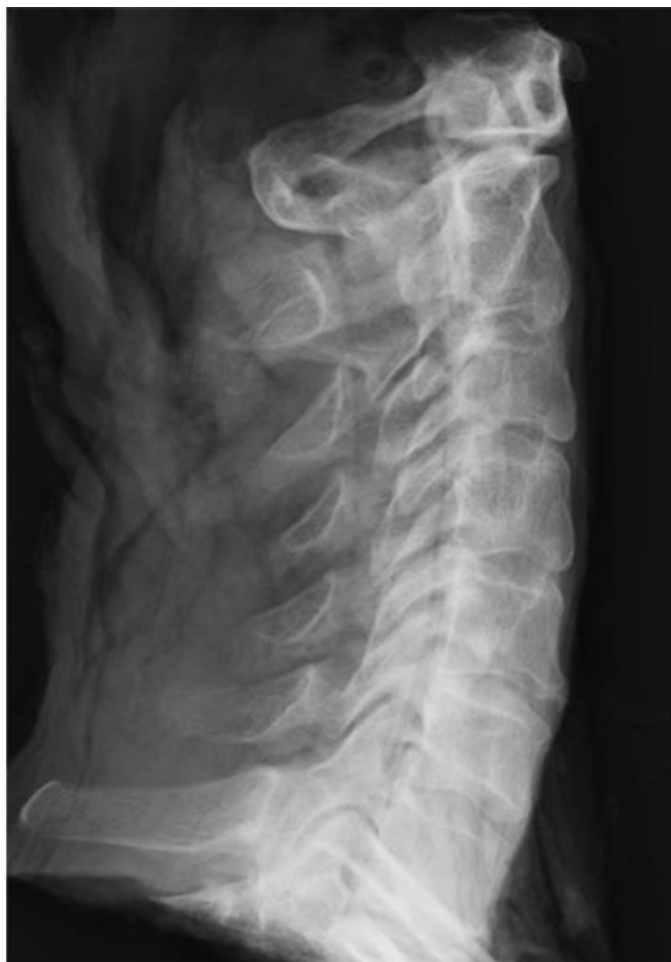

Abb. 2-2: seitliche Aufnahme

Röntgenaufnahme C1 bis Th1 (HGW 10/07) zur Untersuchung auf pathologischer Veränderungen.

Unter dem Erhalt von Bandstrukturen und Gelenkkapseln wurden nachfolgend die Segmente C2-C4 herausgetrennt und Muskeln, sowie Bestandteile des zentralen Nervensystems vollständig abpräpariert. Jedes Präparat erhielt eine individuelle Kennziffer (Tab. 2-1). Unter der Verwendung von Formalin-Ethanol-Glycin-Lösung fand die Fixation des Untersuchungsmaterials statt. Dadurch konnte einer übermäßigen Verhärtung der Strukturen entgegen gewirkt werden (Fanghänel und Schultz 1962, Fanghänel 2009). In Folge dessen blieb die Elastizität des Bandapparates und damit verbunden die kinematischen Eigenschaften erhalten. Um eine Austrocknung der Präparate während des Versuchsablaufes zu verhindern, wurden diese mit Fixationslösung getränkten Kompressen bedeckt und zusätzlich vor und nach jedem Versuch großzügig beträufelt. 


\begin{tabular}{ccc} 
Bezeichnung & $\begin{array}{c}\text { Alter } \\
\text { (in Jahren) }\end{array}$ & Geschlecht \\
\hline HGW 14/05 & 54 & M \\
\hline HGW 16/05 & 80 & W \\
\hline HGW 08/06 & 68 & M \\
\hline HGW 10/06 & 85 & W \\
HGW 13/06 & 83 & W \\
\hline HGW 10/07 & 46 & W \\
\hline HGW 11/07 & 67 & W \\
\hline HGW 16/07 & 75 & W \\
Mittelwert & 69,75 & \\
\hline o & 13,95 & \\
\hline
\end{tabular}

Tab. 2-1: Übersicht Versuchsobjekte;

Anonyme Kennziffer, Alter und Geschlecht des

Körperspenders; M= männlich, W= weiblich; $\sigma=$ Standardabweichung

\subsubsection{Einbettung}

Die Einbettung der einzelnen Versuchsobjekte diente der sicheren Verbindung des Präparates mit der Messapparatur. Um unerwünschte Bewegungen im Bereich des „Junghanns“ schen Segmentes“ C2/C3 zu unterbinden, fand eine Fixierung unter Zuhilfenahme von Schrauben statt. Die Beweglichkeit des Präparates beschränkte sich folglich auf das Segment C3/C4. In den C4-Wirbel wurden zusätzlich Schrauben eingebracht, was einen besseren Halt im Kunststoff garantierte. Der Erhalt des zweiten Halswirbels zielte gleichsam auf diesen Sachverhalt ab. Als Gussform fungierte eine Stahlwanne, in welcher das Versuchspräparat so ausgerichtet wurde, dass der Wirbelkanal zentral im Mittelpunkt der Wanne lag. Zusätzlich einmontierte Flügelmuttern dienten der späteren Verbindung mit der Messapparatur. Schließlich wurde die Gussform mit dem kaltpolymerisierenden Kunststoff Weitur ${ }^{\circledR}$ Press (Standard) gefüllt und jeweils der kraniale und kaudale Abschnitt der Präparate eingebettet. Die korrekte Platzierung der Schrauben und das Einbettungsergebnis sind mittels Röntgen in zwei Ebenen und CT verifiziert worden. Die CT-Schichtung erfolgte mit einer Dicke von ca. 0,6 mm parallel zu den Kunststoffblöcken mit Erfassung dieser, um diese als Referenzierung bei der Auswertung zu nutzen. 


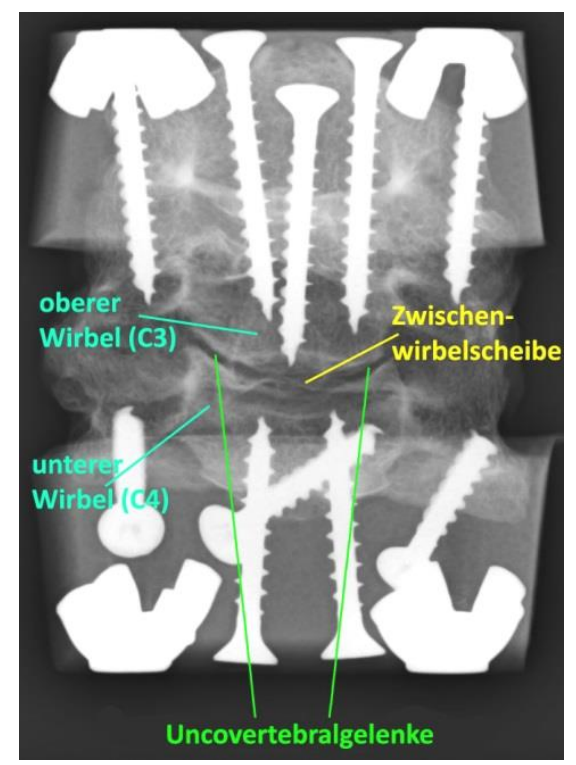

Abb. 2-3: HGW 16/07, ap (Abbildung modifiziert nach Wachowski et al. 2013, Abb. 1)

Röntgenaufnahme eines eingebetteten, intakten Segmentes C2 bis C4 (HGW 16/07). Fixierung der Facettengelenke und des Zwischenwirbelraumes C2/C3 mit Schrauben. Dokumentierung der extraartikulären Lage der Schrauben im Bereich des Segmentes C3/C4. Die eingegossenen Flügelmuttern dienen der starren Verbindung mit der Messapparatur.

\subsubsection{Prothesenimplantation}

Unter Anleitung eines erfahrenen Wirbelsäulenchirurgen (Dr. Dörner, Orthopaedicum NortheimGöttingen) und gemäß den Implantationsanweisungen des Prothesenherstellers (Medtronic Sofamor Danek) erfolgte der Einbau der Bandscheibenprothesen. Jedes Halswirbelpräparat wurde zunächst mit der Prestige ${ }^{\circledR}$ LP bestückt.

Die Resektion des Ligamentum longitudinale anterius auf Bandscheibenhöhe stellte jeweils den primären Schritt dar und eröffnete den Zugang zum Bandscheibenfach. Die anschließende Entfernung des gesamten Bandscheibenmaterials fand unter Zuhilfenahme von Skalpellen und scharfen Löffeln (nach Volkmann) statt (Abb. 2-5). Es folgte die Durchtrennung des Ligamentum longitudinale posterius. Darüber hinaus erforderte die Implantation der Prothese eine geringe Vergrößerung des Bandscheibenfaches durch sparsame Abtragung von Knochenmaterial an den Deckplatten. Zu diesem Zweck kam das vom Prothesenhersteller entwickelte Raspelinstrumentarium zum Einsatz. Um eine sichere Verankerung im Wirbelkörper zu ermöglichen, war es im Zuge dieser Maßnahme obligat, ebene und parallel verlaufende Endplatten herzustellen. Dies wiederum bedingte die partielle Entfernung der Basis der Uncovertebralgelenke. Durch den Einsatz spezieller ImplantatMustervorlagen in das Bandscheibenfach konnten während der Resektionsphase noch störende Strukturen identifiziert und die passende Prothesengröße ermittelt werden. Abschließend wurden jeweils zwei schmale, als Führungsschienen dienende Kiele (Rails) in die ebene Grund- und 
Deckplatte eingeschlagen. Die verwendete Kielstanze („Rail Punch“) muss möglichst zentral in der Mittellinie der Wirbelkörper positioniert werden, um letztendlich einen optimalen Sitz der Prothese zu erreichen (Medtronic 2004, Mummaneni et al. 2007). Die Prestige ${ }^{\circledR}$ LP Cervical Disc, wie auch die Bryan $^{\circledR}$ Cervical Disc, sollte in sagittaler Ausrichtung in der Mittellinie des Bandscheibenfaches implantiert (Traynelis 2011) und so weit eingeschlagen werden, bis die Aufhängung („Tab“) mit der Vorderfläche der Wirbelkörper in Kontakt tritt (Medtronic 2004). Die Größe der Prothese wurde, entsprechend den Herstellerempfehlungen, so gewählt, dass sie mit den Hinterkanten der Wirbelkörper abschloss ohne diese zu überragen. Dabei ist eine Distraktion des Segmentes vermieden worden, indem keine zu große Höhe des Implantates verwendet wurde. Die Kunststoffblöcke verblieben (als zusätzliche Referenz) parallel zu einander (s. Abb. 2-6 und Abb. 4-1).

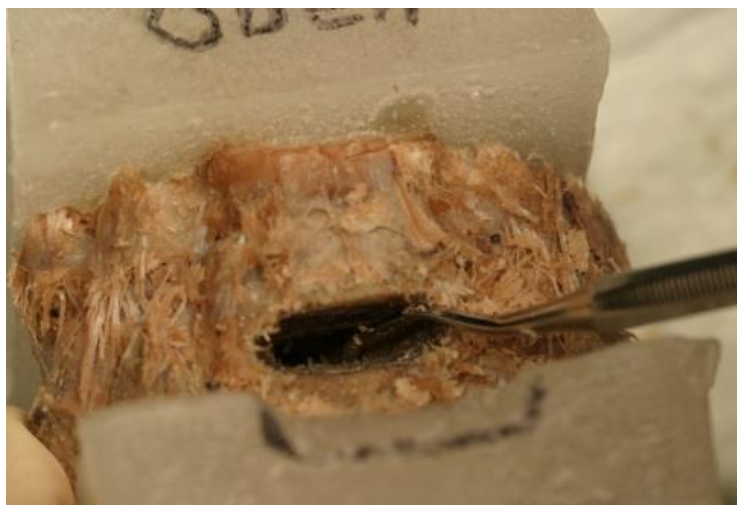

Abb. 2-5: HGW 16/07; Bevor die Prestige ${ }^{\circledR}$ LP Disc eingesetzt worden ist, wurde das Bandscheibengewebe mittels geeigneten Instrumentariums vollständig entfernt.
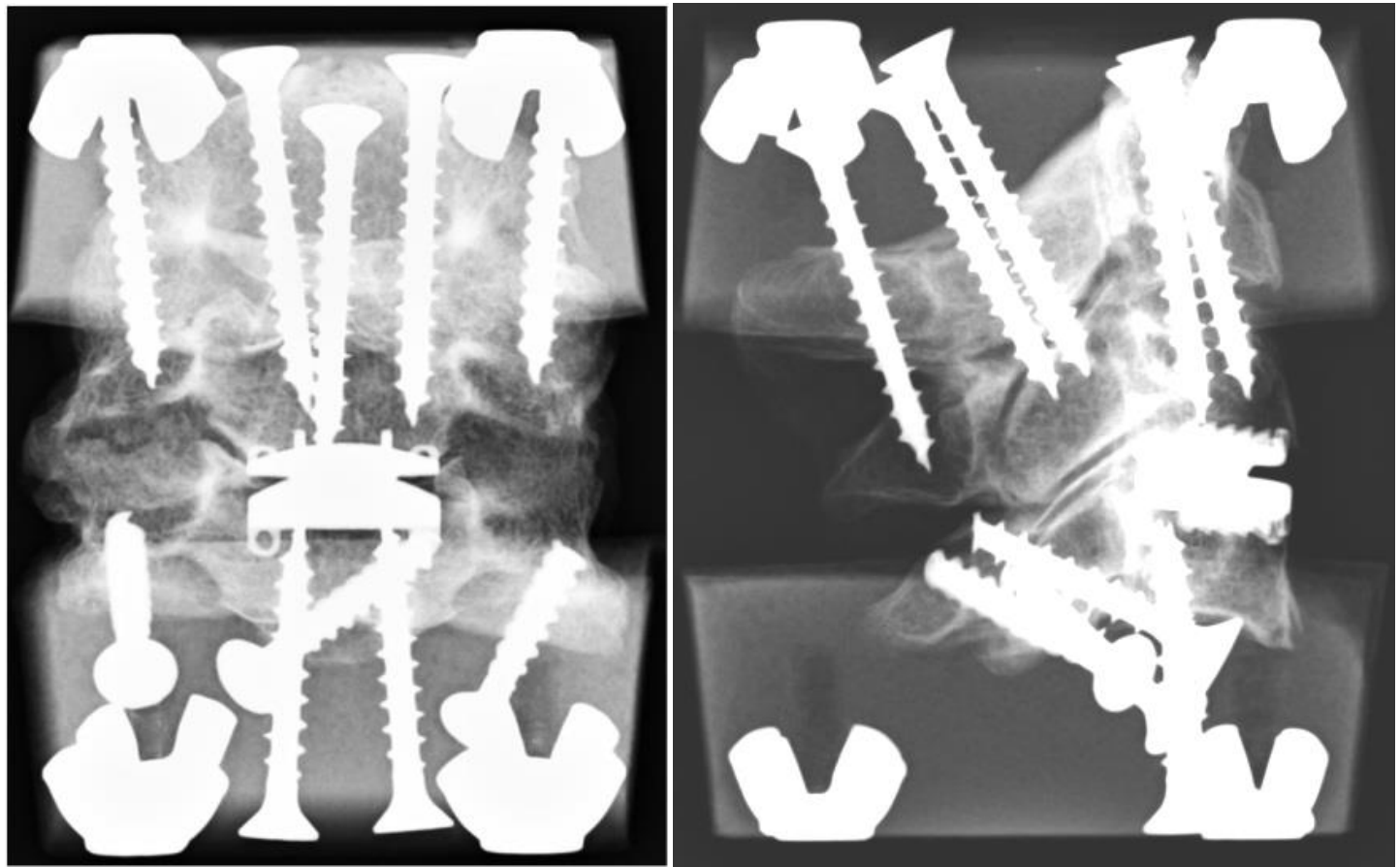

Abb. 2-6: HGW 16/07; Röntgenbild nach Implantation der Prestige ${ }^{\circledR}$ LP. Die Bandscheibenprothese sitzt mittig im Bandscheibenfach und wurde bis an den Spinalkanal bündig eingeschlagen. Ventral schließt Sie eben mit der Vorderkante der Wirbelkörper ab. Die Parallelität der Kunststoffblöcke im seitlichen Bild zeigt, dass das Segment nicht in die Extension distrahiert worden ist (Abbildung modifiziert nach Wachowski et al. 2013, Abb. 1) 
Die Nutzung derselben Wirbelsäulenpräparate für die Untersuchung von Bryan ${ }^{\circledR}$ Cervical Disc war dadurch möglich, dass laut Herstellervorgaben zur Schaffung des Prothesenbettes ein 8,65 mm weiter Zwischenwirbelraum geschaffen werden muss. Für die Prestige-Implantation war eine geringere Knochenresektion nötig, so dass die Bryan-Prothese als zweites regelrecht implantiert werden konnte.

Die erste Maßnahme war folglich die Entfernung der Prestige ${ }^{\circledR}$ LP Disc aus den Versuchspräparaten. Im Anschluss fanden erneut Raspelinstrumente, die allerdings eine höhere Stärke aufwiesen, ihre Anwendung. In Folge dessen wurde der Raum zwischen den Wirbeldeckplatten standardgemäß auf 8,65 mm vergrößert, sodass die eingeschlagenen Führungsfurchen der Prestige ${ }^{\circledR}$ LP Disc verstrichen. Mit Hilfe von Implantat-Mustervorlagen definierte man erneut die passende Prothesengröße. Die Endplatten der Wirbelkörper mussten nun, entsprechend der linsenförmigen Deckplattenform der Bryan $^{\circledR}$ Disc, mittels einer speziellen Scheibenfräse bearbeitet werden. Um ein präzises und ortsstabiles Abtragen des Knochenmaterials sicher zu stellen, fand gemäß den Vorgaben des Herstellers die vorübergehende Montage von ventral angebrachten Führungseinrichtungen auf den HWK 3 und 4 statt. Abschließend erfolgte die Implantation der Prothese, deren konvexe Oberflächen festen Halt in den konkav ausgefrästen Mulden der Wirbelendplatten fanden. Auch hier erfolgte die Präparation in der Art und Weise, dass keine Distraktion des Segmentes zu Stande kam (s. Abb. 2-8 und Abb. 4-1).

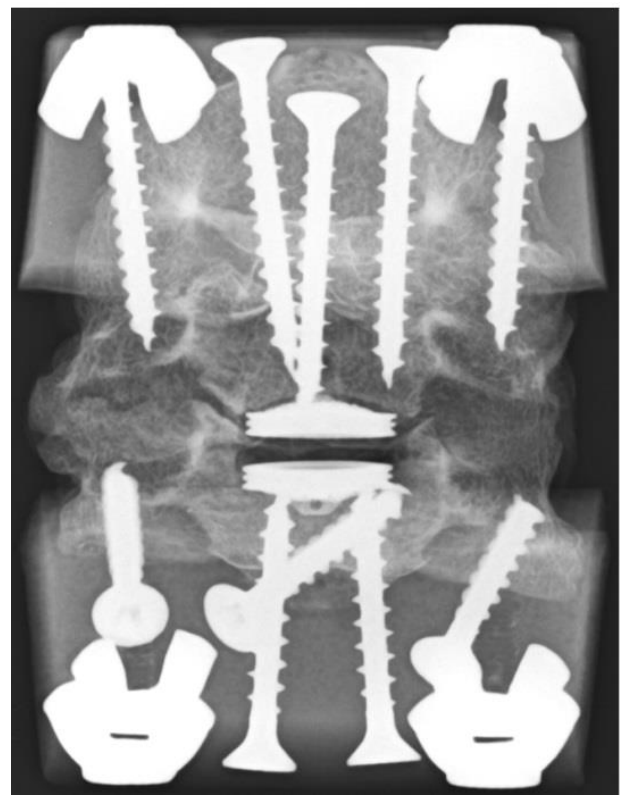

Abb. 2-7: HGW 16/07 mit Bryan® Disc, ap (Abbildung modifiziert nach Wachowski et al. 2013, Abb. 1)

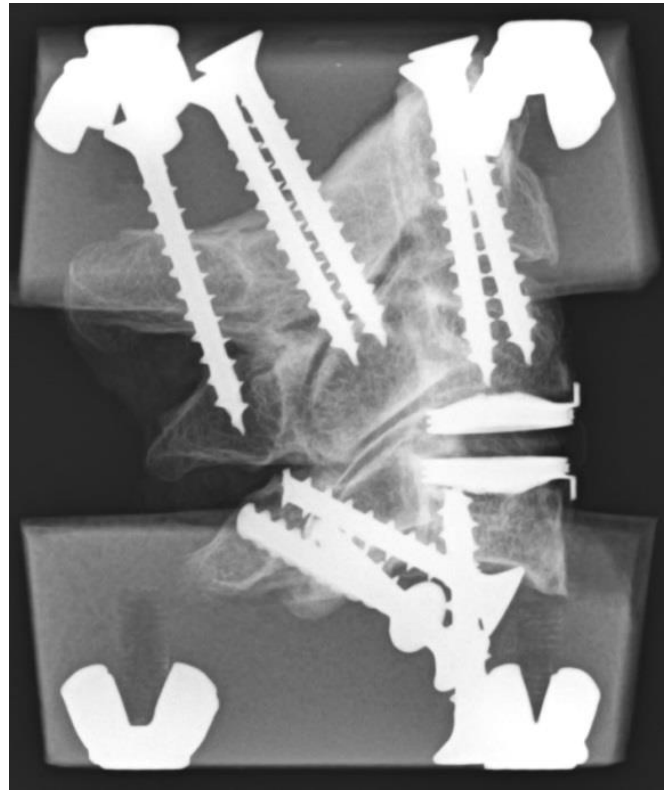

Abb. 2-8: HGW 16/07mit Bryan® Disc, seitlicher Strahlengang

Röntgenaufnahmen nach Implantation der Bryan ${ }^{\circledR}$ Disc in ap und seitlichem Strahlengang. Wie die Prestige ${ }^{\circledR}$ LP, sitzt die Bryan-Bandscheibenprothese mittig im Bandscheibenfach (Abb. 2-7) und wurde bis an den Spinalkanal bündig eingeschlagen. Ventral schließt Sie mit der Vorderkante der Wirbelkörper ab (Abb. 2-8). 


\subsection{Messsystem}

\subsubsection{Messapparatur}

Das stählerne Grundgerüst der verwendeten Apparatur ist aus Vierkantstangen aufgebaut. Mittig auf diesem Unterbau befindet sich eine $50 \times 50 \mathrm{~cm}$ große Stahlplatte, welche das eigentliche Messsystem (Spiering 1995) trägt. Das gesamt Konstrukt wurde auf Schaumstoff gelagert und sämtliche Gerüststangen mit Schaumstoffröhren ummantelt, wodurch das Messsystem vor äußeren Schwingungen isoliert war und Eigenschwingungen im Verlauf einer Messung abgedämpft wurden. Das in die untere Pfanne eingesetzte Versuchspräparat wurde mittels vier Schrauben fest an der Grundplatte fixiert. Anschließend erfolgte die Montage eines Stahlkreuzes an den oberen Objektabschnitt, welches der Applikation des Drehmomentes und von unterschiedlichen Vorlasten diente. Das Eigengewicht des Drehkreuzes wurde über ein umgelenktes Gegengewicht kompensiert und wirkte folglich absolut kräftefrei auf das Material. Eine an diesem Kreuz aufgehängte Schiene ermöglichte die Anlage der Vorlast, welche über ein frei hängendes Gewicht ausgeübt und in sagittaler und transversaler Richtung verschoben werden konnte. Die Applikation des Drehmomentes erfolgte mittels einer festgelegten Wassermenge, die zwischen zwei Behältern hin und her gepumpt wurde. Dabei war das Pumpsystem auf einem separaten Ständer angebracht, um die Übertragung von Schwingungen auf das Messsystem zu vermeiden. Die Behälter wurden über Seilzüge wiederum mit dem Kraftkreuz verbunden und deren Gewichtskraft durch Gegengewichte kompensiert. Verschieden positionierte Rollen ermöglichten die Umlenkung der Seilzüge und gewährleisten die Ausübung des Drehmomentes in $T_{x^{-}}, T_{y^{-}}$und $T_{z^{-}}$

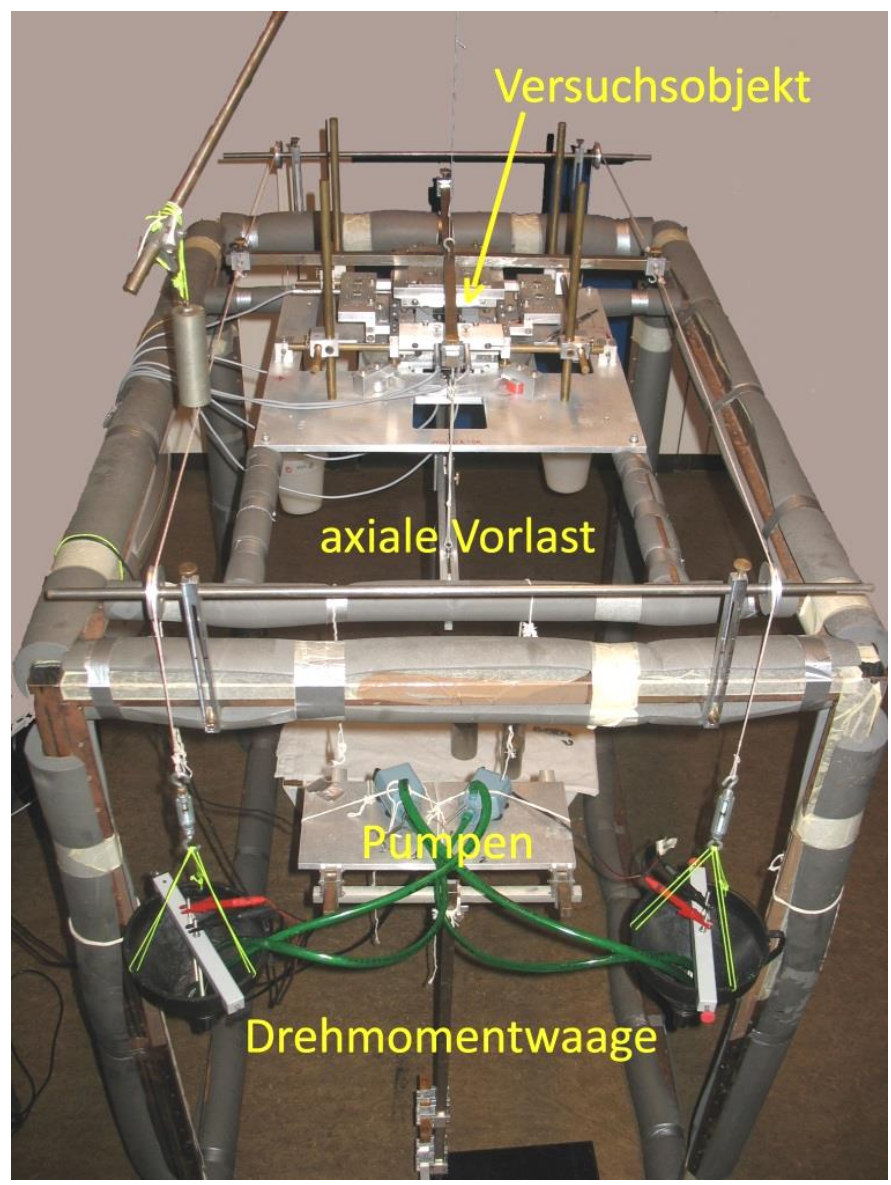

Abb. 2-9: Messapparatur; Aufbau zur Messung der biomechanischen Eigenschaften eines Präparates bei axialer Rotation.

Richtung. Im Verlauf eines Pumpzyklus führte die Ab- bzw. Zunahme der Wassermenge in den Gefäßen nun zur Modifikation der Zugkräfte und induzierte somit eine Bewegung des oberen Wirbels. Diese Veränderung wurde mit Hilfe von sechs Tastern gemessen, die in einer 
Haltevorrichtung rund um das Versuchsobjekt befestigt waren. Dabei waren die Lagesensoren so angeordnet, dass die Taster 1, 2 und 3 senkrecht auf der $x$-y-Ebene standen und deren Verschiebung registrierten. Die Taster 4 und 5 wurden senkrecht zur y-z-Ebene angeordnet, Taster 6 senkrecht zur x-z-Ebene (3-2-1 Anordnung). Die Ermittlung der Längenänderung der einzelnen Taster und die Verrechnung der Daten ermöglichten nun die Bestimmung der absoluten Lageänderung im Raum (vgl. Einleitung Kapitel 1.5.1).

\subsubsection{Applikation von Kräften}

\subsubsection{Axiale Vorlast}

Die Ausübung axialer Kräfte simulierte neben der bloßen Gewichtskraft des Kopfes, die auf die Halswirbelsäule wirkt, auch verschiedene Kopfhaltungen. Dies wurde durch die Verlagerung der Vorlastposition nach ventral, dorsal oder lateral realisiert. Darüber hinaus diente sie in Kombination mit den angelegten Drehmomenten der Applikation verschiedener Kraftschraubenkonstellationen.

Wie unter 2.3.1 bereits beschrieben, erfolgte die Applikation von axialen Kräften über eine Schiene, deren Enden an den jeweils gegenüberliegenden Armen des Kraftkreuzes aufgehängt waren. Demnach konnte die Metallschiene entweder parallel zur x-Achse (sagittal) oder parallel zur y-Achse (transversal) ausgerichtet werden (Abb. 2-11). Der Balken war mit einem Millimetermaß ausgestattet und verfügte über einen verschiebbaren Reiter, wodurch eine variable und exakte Positionierung der Vorlast ermöglicht wurde. Das Eigengewicht der Schiene bewirkte nunmehr eine konstante, axial im Mittelpunkt der Apparatur wirkende Kraft von $10 \mathrm{~N}$. Weiterführend konnten verschiedene Gewichte an den Reiter angehängt und in sagittaler und transversaler Richtung verschoben werden. Die festgelegten Vorlastpositionen wurden jeweils in Bezug auf die Position des Widerstandszentrums (WZ) gewählt. Diese sind in Abbildung 2-10 und unter dem Punkt 2.10 - „Ablauf der Messung“ dargestellt. Für jedes Präparat war die Position des Widerstandszentrums zuvor

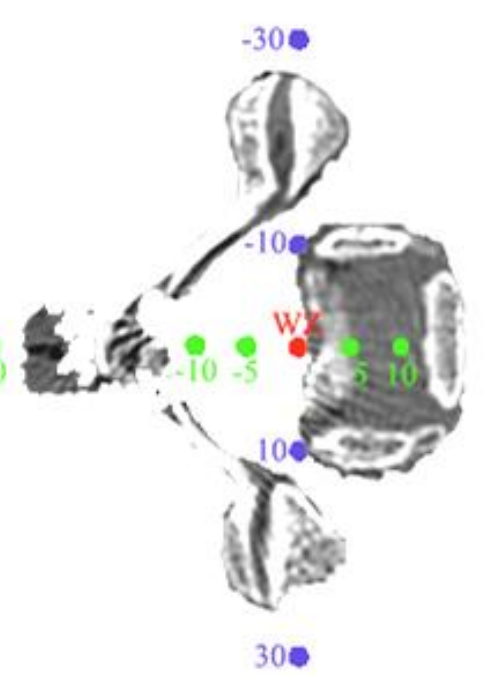

Abb. 2-10: Positionen der axialen Vorlast, ausgehend vom Widerstandszentrum WZ (Angabe in Millimeter). Die Lagebeziehung wird durch einen Horizontalschnitt auf Höhe der Bandscheibe (hier HGW 10/07) veranschaulicht. Grün: sagittale Verschiebung der VL; blau: transversale Verschiebung der VL

experimentell ermittelt worden (siehe 2.7 Bestimmung des Widerstandszentrums). 


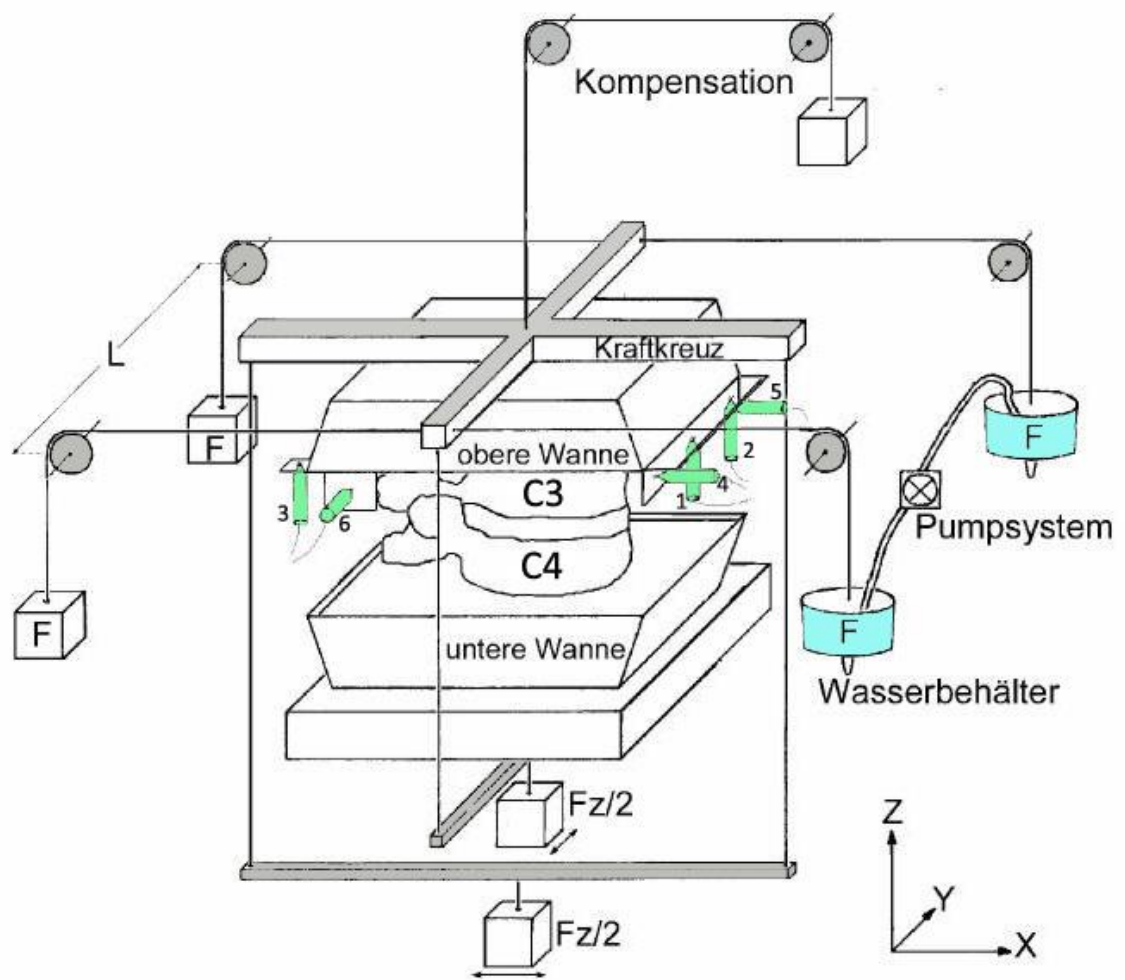

\subsubsection{Drehmoment}

Die Applikation eines Drehmomentes wurde mit Hilfe eines Pumpsystems, welches eine definierte Wassermenge von $800 \mathrm{ml}$ zwischen zwei Behältern hin und her pumpte, realisiert. Nachfolgend wird die Ausübung eines axialen Drehmomentes ( $T_{2}$-Richtung) genauer beschrieben, welches einer Rotation des Kopfes von links nach rechts und umgekehrt entspricht.

An die parallel zur y-Achse liegenden Arme des Kraftkreuzes wurden vier Seile befestigt, die in einem $90^{\circ}$-Winkel zum Hebelarm (Länge: $28 \mathrm{~cm}$ ) antiparallel ausgerichtet waren. Demnach verliefen jeweils zwei Seile nach ventral und nach dorsal. In einem Abstand von mindestens $50 \mathrm{~cm}$ erfolgte die Befestigung von kugelgelagerten Umlenkrollen am Apparaturrahmen, über welche die Seilzüge umgelenkt wurden (siehe Abb. 2-11). Diese Rollen wiesen lediglich einen minimalen Widerstand auf. An den Enden der Seile waren gleichschwere Wasserbehälter aufgehängt. Es lag demzufolge ein Gleichgewicht vor, da sich die Gewichtskräfte gegenseitig aufhoben und weder Zugkräfte noch Drehmoment auf das Segment wirkten. Dorsal des Präparates, wurde das Pumpsystem aufgebaut. Das Wasser konnte dadurch zum Beispiel vom linken Behälter in den rechten gepumpt werden. Infolgedessen entstand ein zyklisches Drehmoment, was sich in diesem Fall (Drehmoment in negative z-Richtung) in einer Rechtsdrehung äußerte. Analog dazu resultierte eine Linksdrehung, wenn das Wasser aus dem rechten Behälter in den linken gepumpt wurde (Drehmoment in positive z- 
Richtung). Bei dieser Studie kamen zwei „Rena Flow 300C“ Kreiselpumpen, mit einer Förderleistung von 2,44 I/min, zum Einsatz, welche luftleer betrieben wurden. Die Pumpen arbeiteten in entgegengesetzter Förderrichtung, d.h. dass die erste Pumpe Wasser vom linken in den rechten Behälter, die zweite Pumpe vom rechten in den linken pumpte. Um einen automatischen Wechsel der Pumprichtung zu ermöglichen, wurde ein elektronisches Steuerungssystem installiert. Dieses bestand aus je zwei Elektroden, welche unterschiedlich tief in die Behälter ragten (Abb. 2-13). Sank der Wasserstand im Laufe eines Pumpzyklus unter die erste Elektrode, brach der Stromfluss zwischen den elektrischen Kontakten ab. Der Widerstand zwischen den Elektroden erhöhte sich und das Steuergerät schaltete auf die andere Pumpe um. Auf diese Weise war die Umschaltung der Pumprichtung jederzeit reproduzierbar. Dies gewährleistete zudem eine Restwassermenge von 55 $\mathrm{ml}$, die beim Umschalten des Pumpzyklus im entsprechenden Behälter verblieb. Ein Pumpzyklus war vollendet, wenn die komplette Wassermenge $(800 \mathrm{ml})$ von einem Wasserbehälter in den anderen befördert wurde. Ein Messzyklus hingegen beinhaltete zwei Pumpzyklen, d.h. das Wasser wurde einmal hin und zurück gepumpt. Daraus resultierte eine maximale Rotation des Segmentes aus kompletter Linksdrehung nach rechts/links, und anschließend in die Ausgangsstellung zurück (zweiter Pumpzyklus).

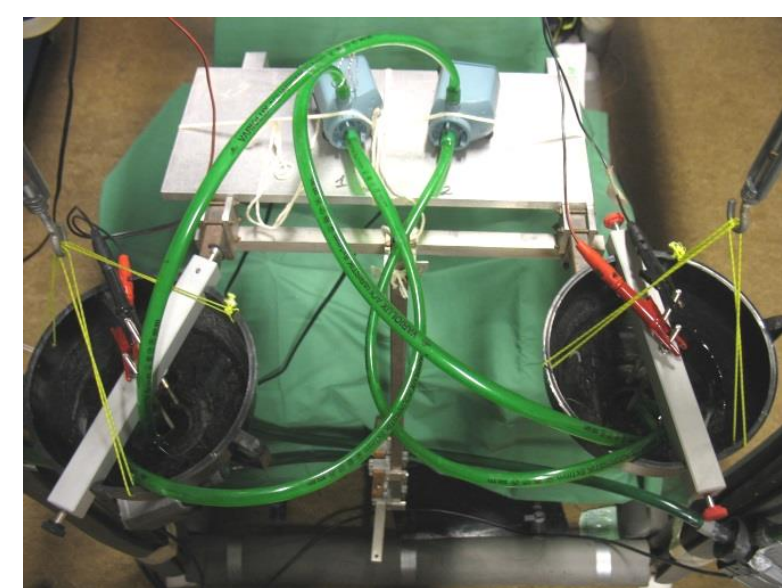

Abb. 2-12: Pumpsystem mit Wasserbehältern. Die beiden Pumpen (oben) befördern die Flüssigkeit zwischen den beiden Behältern hin und her.

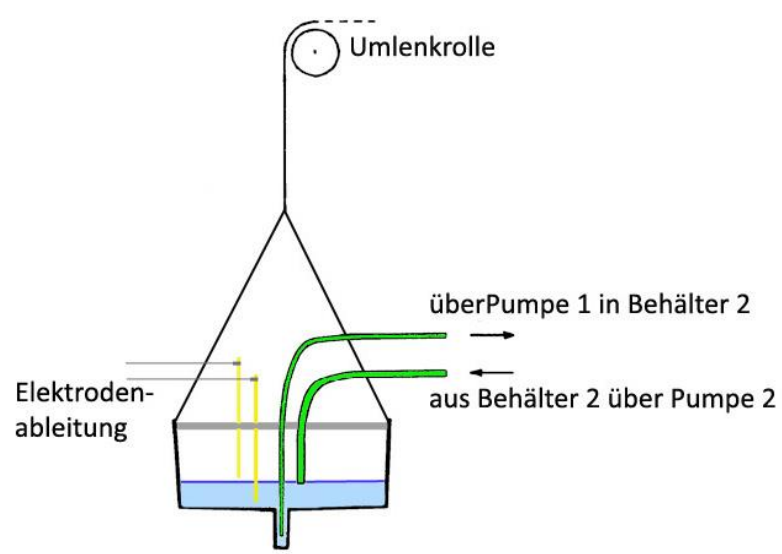

Abb. 2-13: Schema des Leitungssystems eines Wasserbehälters. (modifiziert nach Mansour 2001, S. 60) 
Die Applikation des Drehmomentes in x-Richtung erfolgte nach dem gleichen Prinzip. Das Wasser wurde zwischen zwei Behältern hin und her gepumpt, welche nun allerdings direkt an den Enden der Kraftkreuzarme in $y$-Richtung angebracht waren. Die Seilzüge verliefen senkrecht nach oben und wurden dort wiederum über kugelgelagerte Rollen umgelenkt. Die Gegengewichte am Ende der Seile glichen die Gewichtskraft der Pumpbehälter aus. Dadurch war das System erneut kräftefrei (Abb. 2-14).

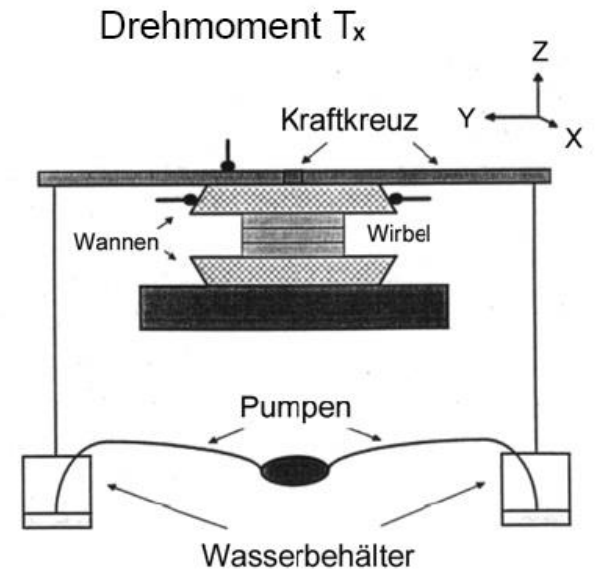

Abb. 2-14: Schema des Versuchsaufbaus zur Applikation eines Drehmomentes in $\mathrm{X}$-Richtung (Mansour 2001, S. 60)

Die Ausübung eines Drehmomentes in y-Richtung war gleichartig. Die Wasserbehälter des Pumpsystems wurden lediglich an den in x-Richtung verlaufenden Armen des Kraftkreuzes befestigt. In den durchgeführten Versuchen betrug das applizierte Drehmoment grundsätzlich 2,25 Nm. Die Resektion der Ligamenta Iongitudinalia anterius und posterius im Rahmen der Prothesenimplantation, führte jedoch zu einem hohen Verlust an Stabilität, was eine Reduktion des angelegten Drehmomentes $T_{y}$ nach TDA auf Werte von ca. 1,7 Nm bzw. 0,85 Nm nötig machte.

\subsubsection{Taster, Messinterface und Software}

Die Lageänderung der beiden Wirbel zueinander wurde in dieser Studie mit Hilfe von Längenmesstastern der Firma Mahr GmbH Göttingen (Typ 1310) gemessen (Abb. 2-15). Diese verfügten über ein Auflösungsvermögen von 0,01 $\mu \mathrm{m}$ und arbeiten nach dem Prinzip der induktiven Längenmessung. Im Inneren des Tasters befindet sich eine Feder, auf die der dünne Tasterstift gelagert ist. Die Spannkraft der Feder wurde möglichst reduziert, um eine Führung des Tasters durch die Feder bestmöglich zu minimieren. Die Taster waren in einem Rahmen um das Versuchsobjekt angeordnet und deren abgerundete Köpfe an Glasplättchen angelegt, die am Kraftkreuz befestigt wurden (Abb. 2-16). Das gläserne Material der Messflächen gewährleistete eine stabile und plane Oberfläche, verhinderte eine elastische Verformung und minimierte die Reibung und den Anpressdruck. Vor Beginn einer Messung wurden die Taster auf halbe Federspannung (Mittelposition) eingestellt, wodurch sowohl eine Verkürzung des Tasters (positive Auslenkung), als auch eine Verlängerung des Tasters (negative Auslenkung) ermittelt werden konnte. Während eines Messzyklus wurde die Längenänderung eines jeden Lagesensors simultan erfasst, in Spannungswerte umgewandelt und über das Messinterface-System Millimar X1715 der Firma Mahr aufgenommen, modifiziert und an einen Computer weitergeleitet. Mit der genutzten Software Millimar D 1000x konnte das Messinterface-System gesteuert werden. Die Verwendung eines auf LabView ${ }^{\circledR}$ (National Instruments) 
basierenden, speziell erstellten Programmes ermöglichte die Darstellung und Auswertung der aufgenommenen Daten, wodurch die Verschiebung des Wirbels analysiert wurden.

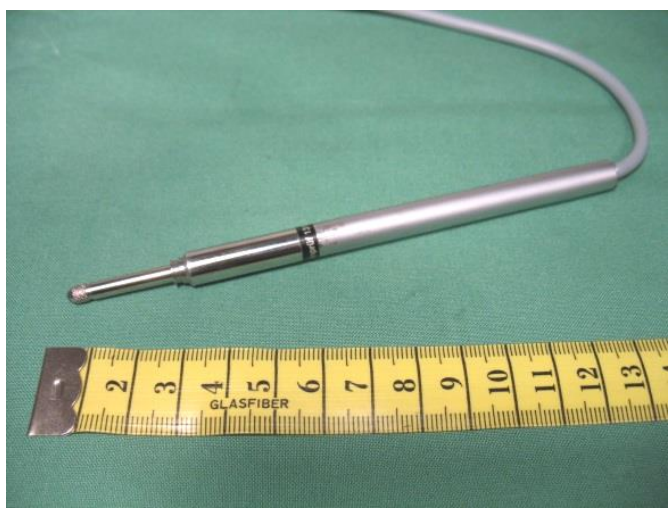

Abb. 2-15: Verwendeter Längenmesstaster der Firma Mahr GmbH Göttingen (Typ 1310).

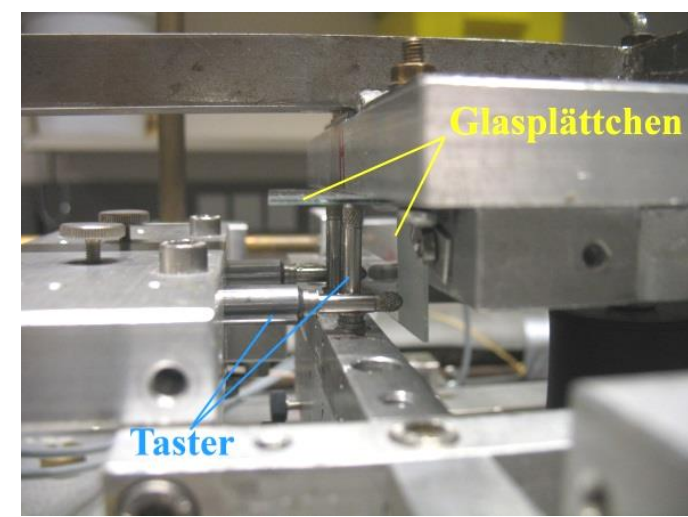

Abb. 2-16: Anordnung der Taster in der Messapparatur. Die Köpfe der Taster liegen Glasplättchen an.

\subsection{Positionsänderung im Raum - Konzept der Schraubachse}

Die Lage eines Körpers im Raum besitzt sechs Freiheitgrade. Um die Positionsänderung des oberen Wirbels (bewegter Körper) zu ermitteln, wurden 6 der unter 2.3.3 beschriebenen Messtaster verwendet. Im rigiden Teil der Apparatur war das Koordinatensystem $\mathrm{K}_{0}$ definiert, welches als Ausgangpunkt für die Bestimmung der Lageänderung des bewegten Objektes diente. Der freigängige Teil am Drehkreuz war fest mit einem zweiten Koordinatensystem $K_{n}$ verbunden.

Die Lagesensoren maßen die Position von $K_{n}$ in Relation zum Referenzsystem $\mathrm{K}_{0}$. Wie unter 2.3.1 beschrieben, waren die Taster dazu in einer 3-2-1 Anordnung angebracht. Die Taster 1, 2 und 3 spannten dabei die xy-Ebene des Koordinatensystems auf. Die Taster 4 und 5 ermittelten die Lage der senkrecht zur xy-Ebene stehende yz-Ebene. Der Taster 6 ermittelte die senkrecht zur xy- und yz- Ebene stehende zx-Ebene. $\mathrm{Zu}$ Beginn einer Messung $\left(t_{0}\right)$ lagen die beiden Systeme $K_{0}$ und $K_{n}$ zunächst übereinander. Die Messtaster standen zu diesem Zeitpunkt auf null. Die Applikation von Kräften auf das System induzierte eine Bewegung des oberen Wirbels. Dies

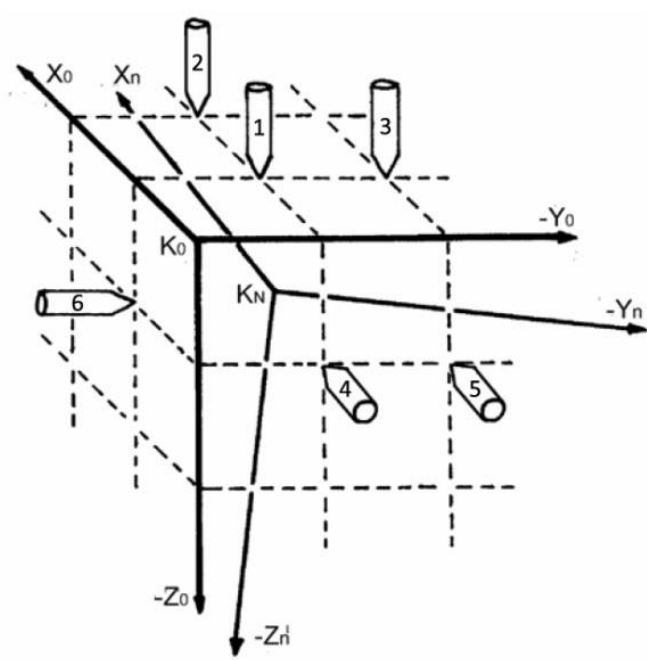

Abb. 2-17: 3-2-1 Anordnung der Messtaster (Prinzip des Messwürfels);

Ursprungskoordinatensystem $\mathrm{K}_{0}$ mit den normierten Achsen $\left(\mathrm{X}_{0},-\mathrm{Y}_{0},-\mathrm{Z}_{0}\right)$; Nach der Bewegung des Messobjekts wird das bewegte Koordinatensystem $K_{n}$ mit den normierten Achsen $\left(X_{n},-Y_{n},-Z_{n}\right)$ bestimmt. (Abb. modifiziert nach Mansour 2001, S. 67) 
führte zu einer Änderung der Sensorwerte und resultierte in einer Lageverschiebung des Koordinatensystems $K_{n}$. Neben dem Bezug zum Ursprungskoordinatensystem $K_{0}$, wurde jede Positionsänderung zu einem Zeitpunkt $t$ dabei zusätzlich in Relation zur Lage des Systems $\mathrm{K}_{\mathrm{n}-\mathrm{1}}$ zum Zeitpunkt t-1 gesetzt. Da jede Bewegung eines Körpers (hier oberer Wirbel) als eine Translation entlang und gleichzeitige Rotation um eine Achse definiert werden kann, ließ sich die Lageänderung des Koordinatensystems $\mathrm{K}_{\mathrm{n}}$ zu $\mathrm{K}_{\mathrm{n}-1}$ als Schraubung um eine momentane Schraubachse beschreiben. Dabei konnte eine räumliche Auflösung von $<0,01^{\circ}$ für Rotation, sowie $<2,4 \mu \mathrm{m}$ für Translationsbewegungen erreicht werden. Mit über 400 errechneten Achsen pro Zyklus wurden differentiell kleine Positionsänderungen erfasst. Daraus wurden die Lage und Ausrichtung der momentanen Schraubachse (IHA, „Instantaneous Helical Axis“) ermittelt. Die Summe aller momentanen Schraubachsen wurde als Schraubachsschar bezeichnet.

\subsection{Bandscheibenmodell}

Anhand eines Bandscheibenmodells, das ähnliche Eigenschaften wie die Bandscheibe aufweist, wurde eine Versuchsreihe im Vorfeld der Hauptmessungen durchgeführt. Es bestand aus einem zylindrischen, viskoelastischen Gummipuffer mit hookeschen Eigenschafen und einem Durchmesser von $40 \mathrm{~mm}$, der zwischen zwei PVC-Platten befestigt war. Diese Konstruktion konnte durch Vorbohrungen in die vorhandenen Stahlwannen eingespannt und in der Messapparatur fixiert werden (siehe 3.1). Analog zur Präzisionsschraube war der Gummipuffer im Mittelpunkt des Messsystems platziert. Die Messungen fanden zunächst unter einer zentral lokalisierten Vorlast von $20 \mathrm{~N}$ (10 N Schiene plus $10 \mathrm{~N}$ verschiebbare Vorlast) statt. Dazu wurde ein Drehmoment von 2,25

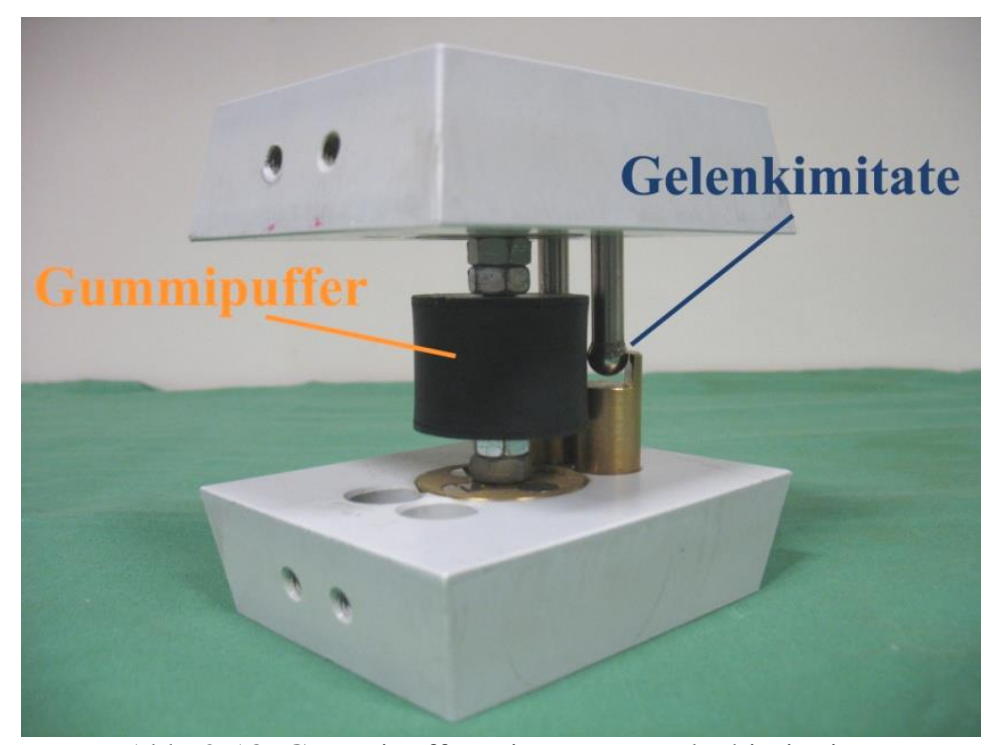

Abb. 2-18: Gummipuffer mit Facettengelenkimitation $\mathrm{Nm}$ angelegt und die Längenänderung der Sensoren aufgezeichnet. Anschließend erfolgte die Untersuchung der Eigenschaften bei ventraler bzw. dorsaler Belastung. In einer weiteren Versuchsreihe wurde das Modell mit speziell geformten Metallbausteinen bestückt. Diese bestehen aus einer Kugel, die bei Bewegung mit einer Metallplatte kontaktiert. Damit entsprach das Konstrukt einer groben Approximierung der Facettengelenke. 


\subsection{Modellmessung Prestige ${ }^{\circledR}$ LP Cervical Disc System}

Um einen Einblick in die biomechanischen Eigenschaften der Prestige ${ }^{\circledast}$ LP Cervical Disc zu erhalten, erfolgte vor der Implantation in die menschlichen Präparate eine separate Vermessung. Dazu wurden die Prothesenteile auf Sockeln fixiert, welche wiederum auf einer PVC-Platte angebracht waren. Die Montage von ebenen Metallbausteinen, dorsal der Prothese, führte zu einer Stabilisierung des labilen Aufbaus und verhinderten ein Verkippen (Versuchsaufbau und Ablauf siehe 2.10).

Der Versuch wurde bei einer zentralen applizierten Vorlast von $60 \mathrm{~N}$ und einem manuell angelegten Drehmoment durchgeführt. Im nächsten Schritt wurden anstelle der ebenen Metallbausteine, die Elemente zur Simulation der Facettengelenke eingebaut und die Messung wiederholt. Die Befestigung in der Apparatur fand analog zu den vorherigen Probemessungen statt. Folglich befand sich die Prothese erneut im Mittelpunkt des Messsystems.

\subsection{Bestimmung des Widerstandszentrums}

Als Widerstandszentrum bezeichnet man den Ort, an dem das Anlegen einer axialen Vorlast keine Verkippung des oberen Wirbels zur Folge hat. Ausgehend von diesem Punkt wurden nach einem festgelegten Schema Vorlasten appliziert (siehe 2.3.2.1). Um das Widerstandszentrum zu finden, wurde neben dem Eigengewicht der Reiterschiene $(10 \mathrm{~N})$ eine zusätzliche Vorlast von $10 \mathrm{~N}$ an unterschiedlichen Positionen entlang der Sagittalachse platziert, woraufhin der obere Wirbel in eine entsprechend gekippte Lage relaxierte. Da es in der Nähe des tatsächlichen Widerstandszentrums zu einer sehr langsamen Verkippung kam, wurde zusätzlich zur angelegten Vorlast ein axiales Drehmoment appliziert, was den Relaxationsvorgang beschleunigte und verstärkte. Die Neigung des C3-Wirbels ließ sich besonders in der xy-Ebene erkennen, die von den Tastern 1, 2, und 3 aufgespannt wird. Die Längenänderung dieser drei Sensoren führte zu einer Verschiebung der Aufzeichnungskurve, was deutlich wurde, wenn man eine Gerade durch die Maxima bzw. Minima der Tasterwerte legte (Abb. 2-19). War die Vorlast hingegen im Widerstandszentrum platziert, blieb eine Verkippung aus. Die eingezeichnete Gerade durch die Extrempunkte der Sensorwerte verlief parallel zur Abszisse (Abb. 2-20). 


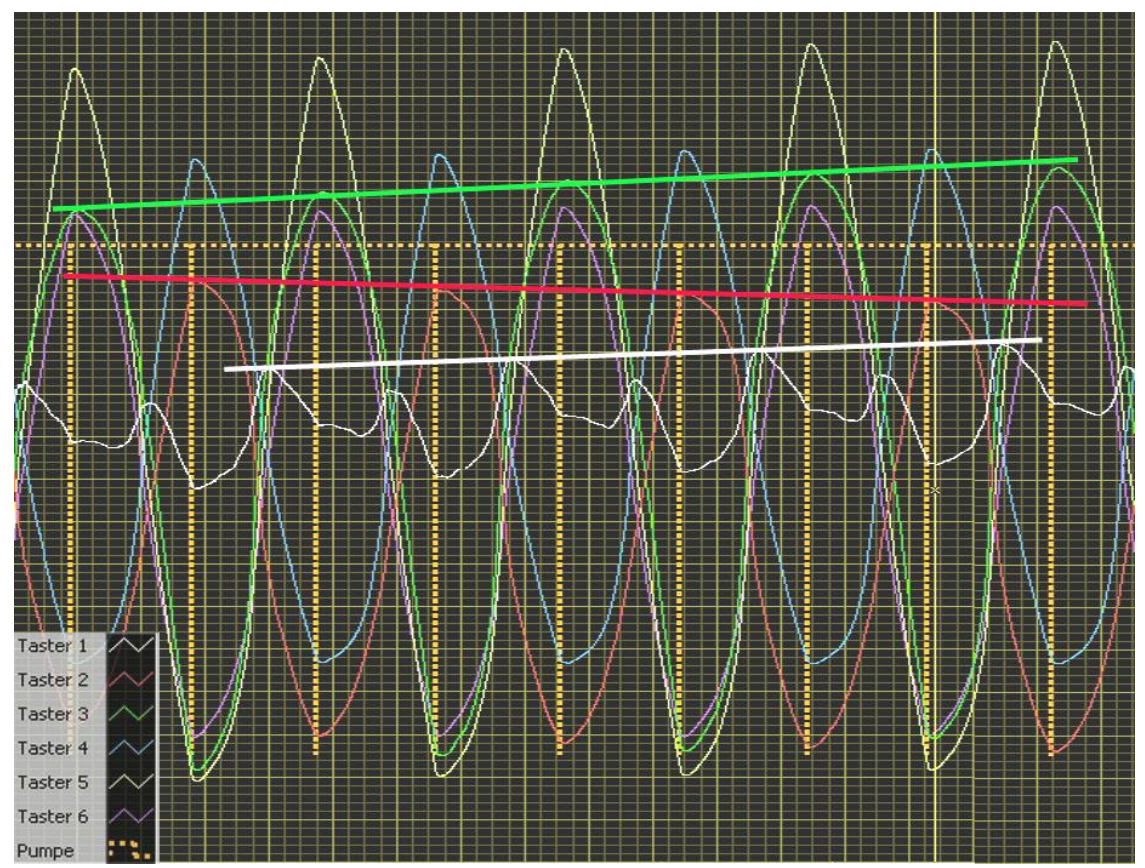

Abb. 2-19: Tasterwerte T1-T6 bei Belastung außerhalb des WZ, mit Tangenten in den Maxima von T1 - T3, die die Ausrichtung der xy-Ebene messen; ventrale VL. Im Verlauf der Bewegung führt die ventrale Vorlast zu einer allmählichen Längenzunahme der Taster 1 und 3; Taster 2 erfährt hingegen eine Längenabnahme. Der obere Wirbel verkippt zunehmend nach dorsal.

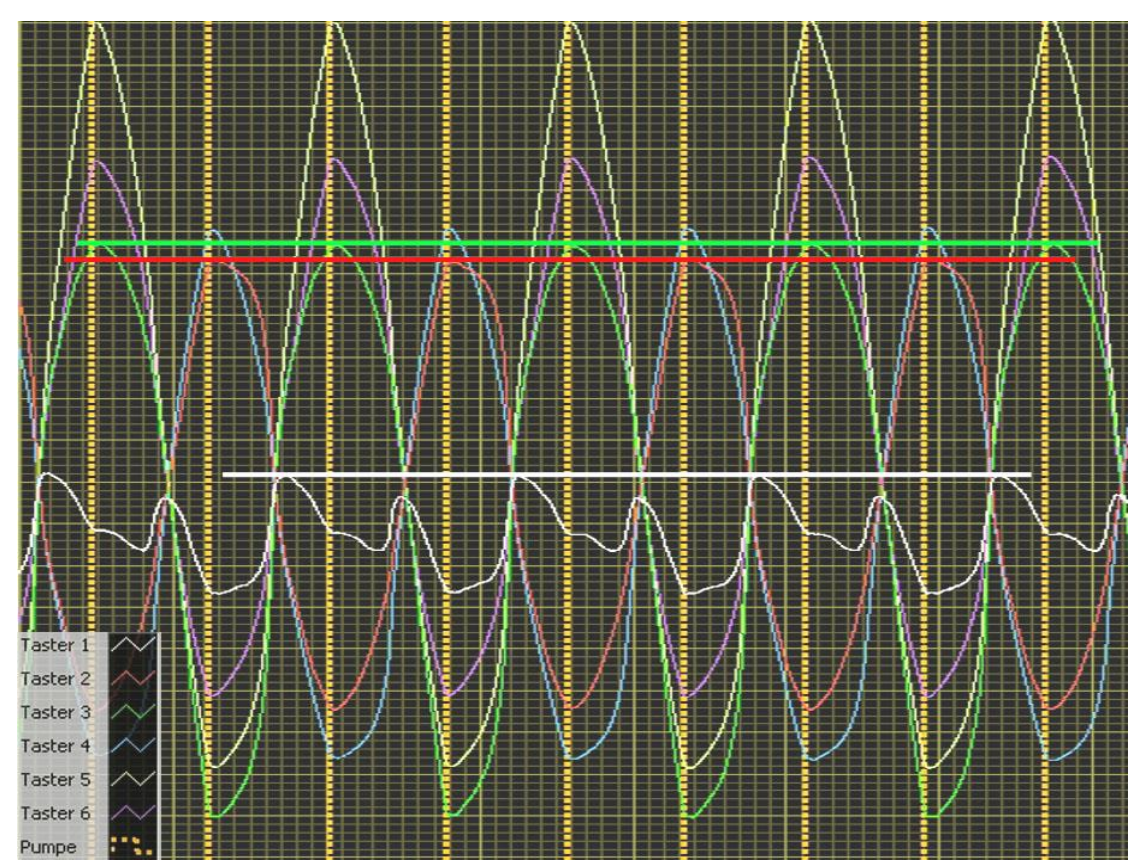

Abb. 2-20: Tasterwerte T1-T6 bei Belastung im WZ; mit Tangenten in den Maxima von T1 - T3, die die Ausrichtung der xy-Ebene messen; VL im WZ. Die Geraden durch die Extremwerte verlaufen parallel zur Abszisse. Eine Längenänderung der Sensoren bleibt aus. Der obere Wirbel verkippt nicht. Die anliegende Vorlast befindet sich damit im Widerstandszentrum. 


\subsection{Graphische Präsentation der Messdaten}

In der vorliegenden Arbeit wurden die gemessenen Parameter der Schraubachse in vier Grafiken visualisiert. Dabei wurden jeweils komplette Messzyklen dargestellt:

\section{Rastpolkurve}

Das Diagramm zeigt die Schnittpunkte $R_{i}=\left(x_{i}, y_{i}, z_{i}\right)$ der momentanen Schraubachsen $\operatorname{dr}(\alpha)$ (IHA; „Instantaneous Helical Axis“ $\left(T_{z}\right)$ ) mit einer definierten Ebene auf Höhe der Bandscheibe an und lässt ihre genaue Position im Verlauf eines Bewegungszyklus erkennen. Zur besseren Veranschaulichung wird die jeweilige CT-Schnittebene des Präparates in die Grafik projiziert. Dadurch ist eine exakte räumliche Zuordnung der Ergebnisse zu den anatomischen Strukturen möglich.

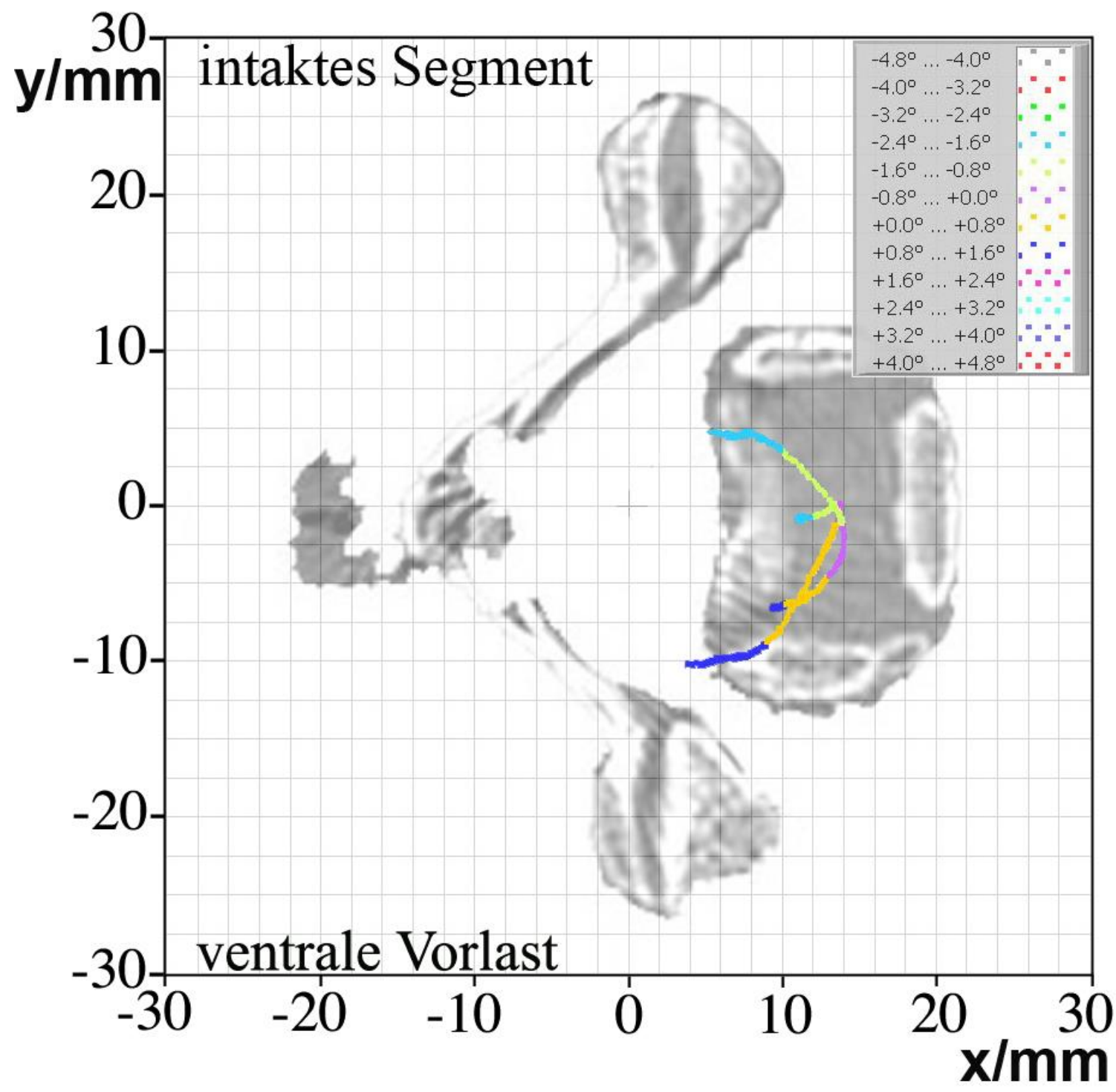

Abb. 2-21: Rastpolkurve; Präparat 10/07, Vorlast: 20 N im Widerstandszentrum; axiale Rotation 


\section{Richtungskomponenten der Schraubachsen}

In diesem Diagramm werden die Komponenten $\left(\begin{array}{llll}e_{x}, & e_{y}, & \left.e_{z}\right) & \text { des }\end{array}\right.$ Richtungsvektors e $(\alpha)$ der momentanen Schraubachse in Abhängigkeit des Drehwinkels dargestellt. Anhand dieser Grafik kann die Ausrichtung der momentanen Schraubachsen während des Bewegungszyklus bei jedem Rotationswinkel quantitativ ermittelt werden. Zur besseren Übersicht wurde der Anfangsbereich der Linksrotation gelb, der Beginn der Rechtsrotation entsprechend violett markiert.

\section{Drehwinkel-Drehmoment-Diagramm}

In dieser graphischen Darstellung (auch $\alpha$ (T)-Diagramm genannt) ist der absolute Drehwinkel $\alpha$ in Abhängigkeit des Drehmoments $\mathrm{T}$ aufgetragen. Es ergibt sich eine Drehwinkel-Drehmoment-Kurve. In diesem Diagramm kann das Bewegungsausmaß des Segments („Range of motion"; ROM) abgelesen werden. Analog zum Diagramm der Richtungskomponenten wurde zur besseren Übersicht der Anfangsbereich der Linksrotation gelb markiert, der Beginn der Rechtsrotation violett.

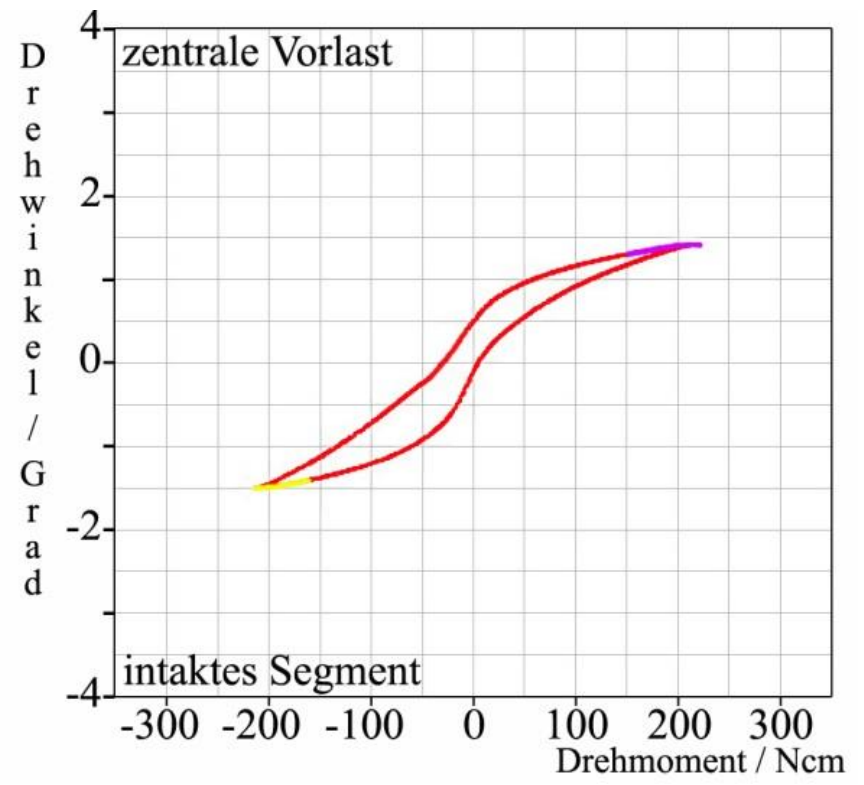

Abb. 2-23: Alpha(T)-Diagramm; Präparat 10/07, Vorlast: $20 \mathrm{~N}$ im Widerstandszentrum; axiale Rotation

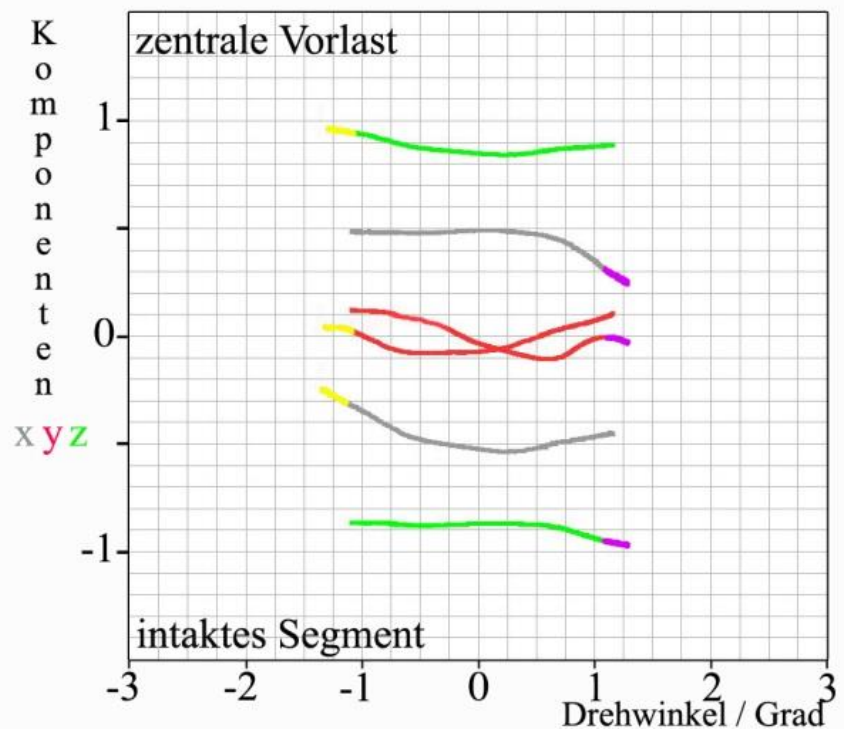

Abb. 2-22: Richtungskomponenten; Präparat 10/07, Vorlast: 20 N im Widerstandszentrum; axiale Rotation 


\section{Steifigkeitsdiagramm}

Die Steifigkeit eines Segmentes entspricht der reziproken Steigung der $\alpha(T)$-Kurve. Im Steifigkeitsdiagramm ist die momentane Steifigkeit $T(\alpha)$ $(\mathrm{Ncm} / \mathrm{deg})$ in Abhängigkeit des Drehwinkels aufgetragen.

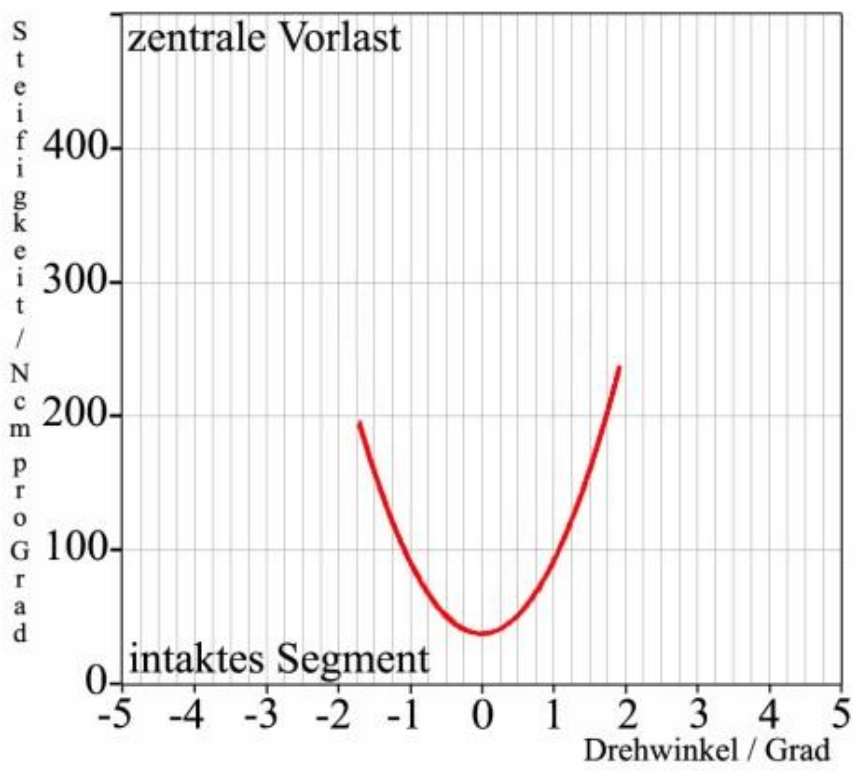

Abb. 2-24: Steifigkeitsdiagramm; Präparat 10/07, Vorlast: $20 \mathrm{~N}$ im Widerstandszentrum; axiale Rotation

\subsection{Statistische Datenanalyse}

Die experimentell ermittelten Daten der untersuchten Parameter wurden im Anschluss an die Vermessung der acht verwendeten Präparate gemittelt und die jeweilige Standardabweichung bestimmt. Die Prüfung signifikanter Unterschiede zwischen dem intakten Segment und der Prestige ${ }^{\circledR}$ LP und Bryan ${ }^{\circledR}$ Cervical Disc erfolgte mittels des Student-Tests (t-Test) und unter Einbeziehung eines erfahrenen Statistikers.

\subsection{Aufbau der Studie}

Vor Beginn der eigentlichen Versuche erfolgte die Validierung der Genauigkeit und der Kalibration des Messsystems mit Hilfe einer Präzisionsschraube. In Vorbereitung auf die Hauptmessungen an den Wirbelsäulenpräparaten wurden anschließend zunächst Modellmessungen an einem Bandscheibenimitat und der Prestige ${ }^{\circledR}$ LP Cervical Disc System durchgeführt und das individuelle Widerstandszentrum eines jeden Präparates experimentell ermittelt.

Zu Beginn der Hauptmessungen wurden die kinematischen Eigenschaften der intakten Segmente erfasst. Dafür wurden verschiedene Vorlasten $(10 \mathrm{~N}, 20 \mathrm{~N}, 50 \mathrm{~N})$ im Widerstandszentrum und nachfolgend eine Vorlast von $10 \mathrm{~N}$ in unterschiedlichen Positionen außerhalb des WZ angelegt. Anschließend erfolgte die Applikation eines zyklischen Drehmomentes an den C3-Körper des Präparates in axialer $\left(T_{z^{-}}\right)$, sagittaler $\left(T_{x}-\right)$ und frontaler Ausrichtung $\left(T_{y}-\right.$ Richtung). Die resultierende Bewegungsänderung wurde sechsdimensional, differentiell klein aufgenommen, analysiert und 
rekonstruiert. Dadurch war es wiederum möglich, die jeweilige Positionsveränderung als momentane Schraubbewegung zu beschreiben und den tatsächlichen Bewegungsvorgang zu erfassen. Die Höhe des Drehmomentes ist experimentell ermittelt worden. Das Drehmoment ist erhöht worden bis eine sigmoide Form des Drehwinkel-Drehmoment-Diagramms zu sehen war, aus deren Abflachung man ersehen konnte, dass das maximale Bewegungsausmaß des jeweiligen Segmentes erreicht war.

Im Anschluss an die Analyse der physiologischen Charakteristika erfolgte die Resektion der Bandscheibe und deren Ersatz mittels Prestige ${ }^{\circledR}$ LP Cervical Disc System, sowie Bryan ${ }^{\circledR}$ Cervical Disc System. Daraufhin wurden die Messungen unter gleichbleibenden Bedingungen wiederholt. Jedoch war aufgrund eines Steifigkeits- und Stabilitätsverlustes eine Reduktion des ursprünglich applizierten Drehmomentes von 2,25 Nm auf 0,84 Nm für die Ventralflexion-Extension erforderlich. Bei der axialen Rotation und Lateralflexion blieb die Stabilität des Systems hingegen soweit erhalten, dass eine Senkung des Drehmoments nicht notwendig war. Nach der statistischen Auswertung und Analyse der kinematischen Eigenschaften beider Bandscheibenprothesen wurden die Ergebnisse mit den Daten der intakten Präparate verglichen und diskutiert. 
$\underline{\text { Studienablauf }}$

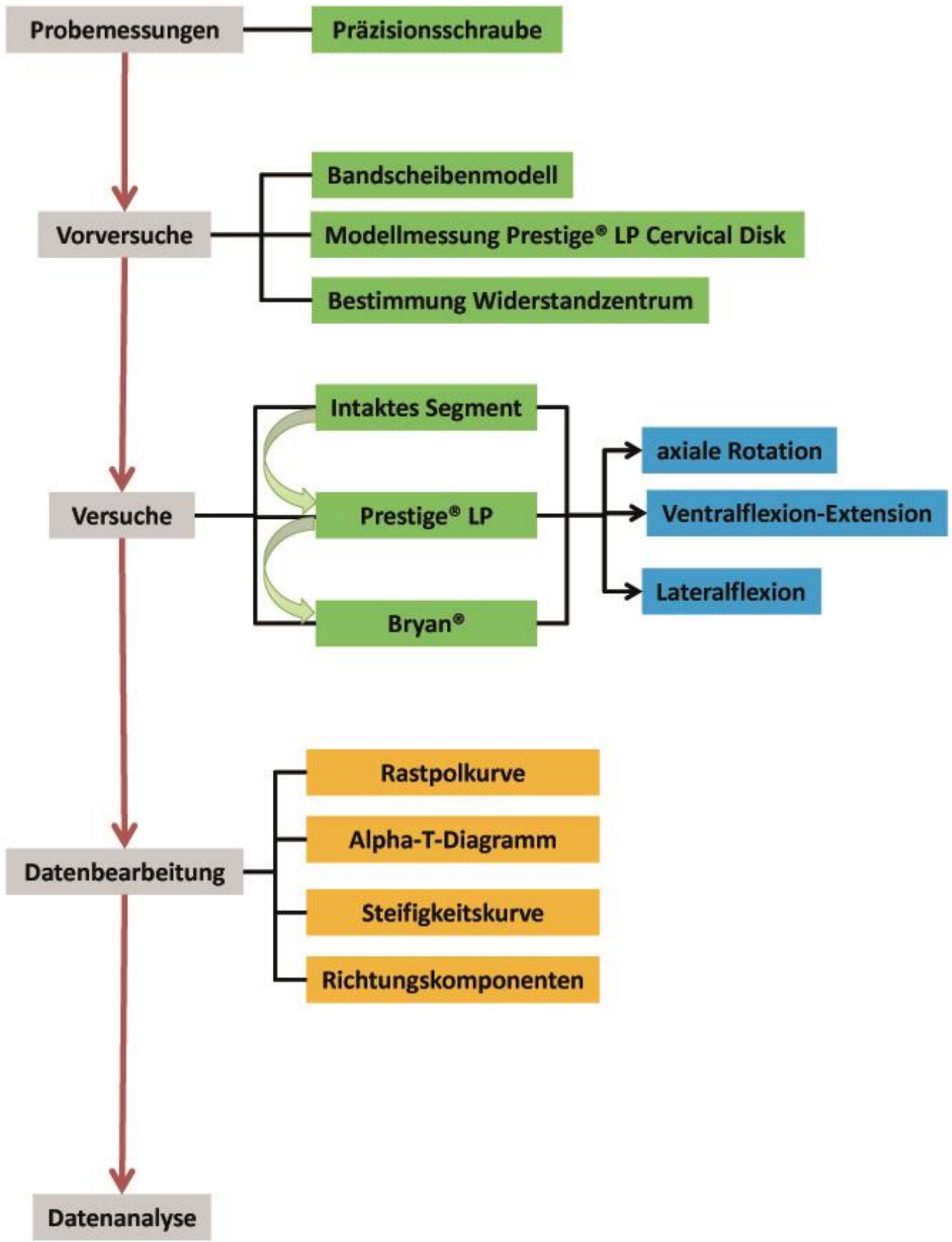

Abb. 2-25: Übersicht: Studienablauf 


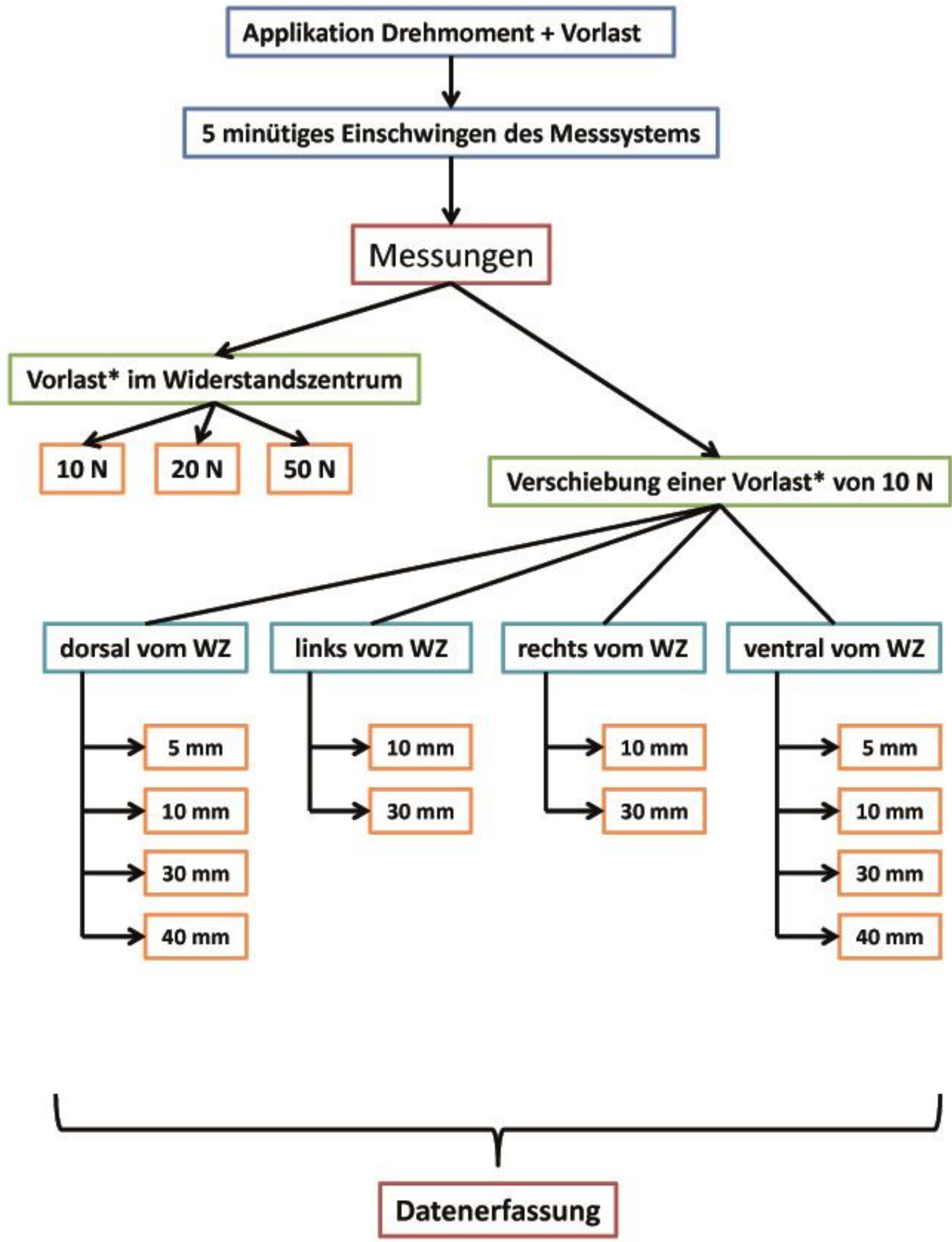

*) zusätzlich zentral wirkendes Eigengewicht der Reiterschiene (10 N) 


\section{Ergebnisse}

\subsection{Probemessung - Präzisionsschraube}

In Vorbereitung auf die Messungen mit biologischen Versuchsmaterialien und zur Überprüfung der verwendeten Messapparatur erfolgten zunächst Experimente an einer Präzisionsschraube. Da deren Eigenschaften bekannt sind, lässt sich anhand der ermittelten Ergebnisse die Genauigkeit sowie Kalibration des Messsystems validieren. Dazu wurde die Schraube, die unverrückbar mit einer Kontermutter an zwei gebohrten Stahlplatten montiert ist, in den Wannen der Messapparatur befestigt und analog zu dem Versuchsaufbau mit den Präparaten im Messsystem fixiert. Die Schraube verlief folglich durch den Apparaturmittelpunkt. Das fertigungsgemäß annähernd schlupffreie Gewinde besitzt eine Schraubsteigung von $0,005 \mathrm{~mm} / \mathrm{Grad}$. Unter Applikation von $100 \mathrm{~N}$ axialer Vorlast und einem manuell angelegten Drehmoment fand eine kontinuierliche Aufzeichnung der Tasterwerte statt.

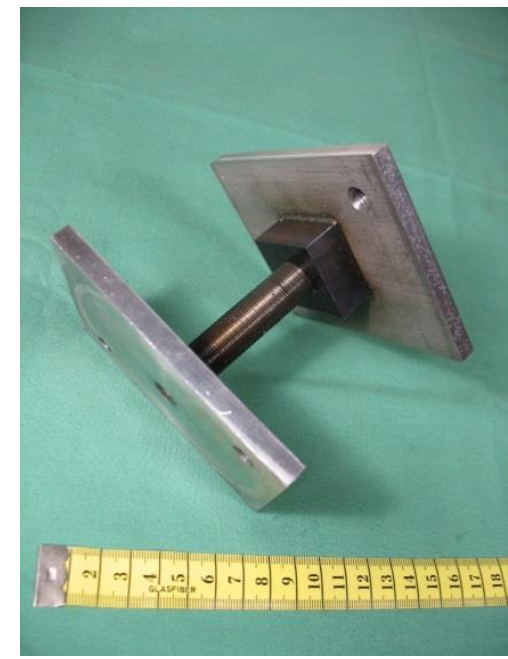

Abb. 3-1: Präzisionsschraube

\section{Ergebnisse}

\begin{tabular}{|l|c|c|}
\hline & gemessen & erwartet \\
\hline Winkel & $8,1^{\circ}$ & $8,13^{\circ}$ \\
\hline Schraubsteigung & $\approx 0,0062 \mathrm{~mm} /{ }^{\circ}$ & $0,005 \mathrm{~mm} /{ }^{\circ}$ \\
\hline Position der Schraubachse & zentral & zentral \\
\hline Ausrichtung der Schraubachse & $z \approx 1($ Abb. 3-3) & z-Richtung \\
\hline Verhalten der Schraubachse & ortsfest (Abb. 3-2) & ortsfest \\
\hline
\end{tabular}

Tab. 3-1: Ergebnisse Präzisionsschraube

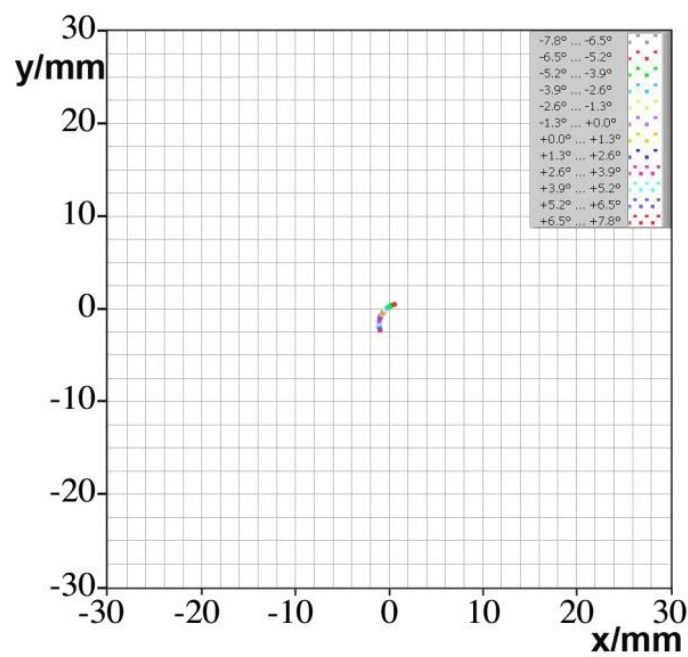

Abb. 3-2: Rastpolkurve: Präzisionsschraube

Die Präzisionsschraube drehte sich um eine ortsfeste Achse, entsprechend der technischen Vorgaben.

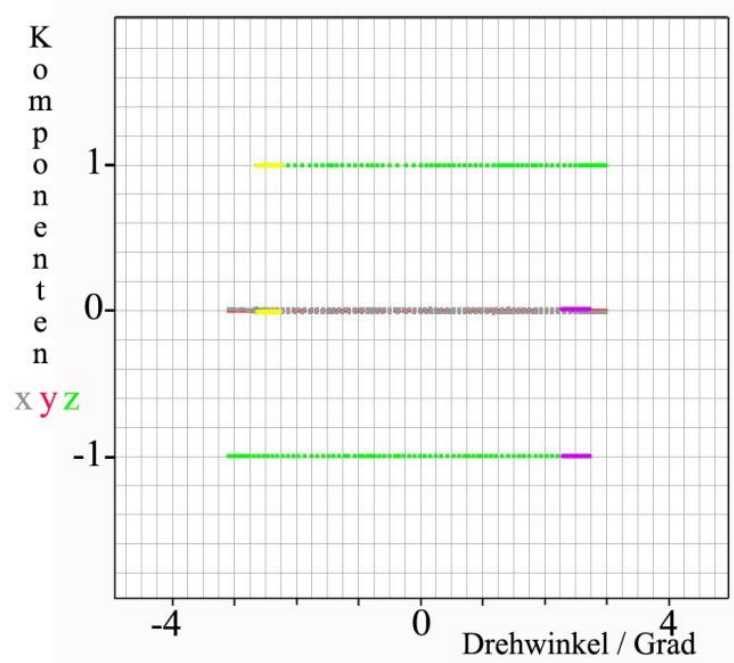

Abb. 3-3: Richtungskomponenten: Präzisionsschraube Die Ausrichtung der Schraubachsen war den gesamten Versuch über senkrecht. 


\section{2 Überprüfung des Pumpsystems}

Für die Applikation des Drehmomentes dienten in dieser Studie zwei Pumpen, die Wasser zwischen zwei Behältern hin und her beförderten. Arbeiten beide Pumpen gleichmäßig, entwickelt sich ein dreiecksförmiges Drehmoment, welches auf das Versuchsmaterial wirkt. Grundlage dafür ist eine konstante Pumpmenge pro Zeiteinheit. Um dies zu überprüfen wurde zunächst eine Gesamtmenge von $1000 \mathrm{ml}$ Wasser transportiert und in Intervallschritten von $200 \mathrm{ml}$ die entsprechend benötigte Zeit bestimmt (Abb. 3-4). Anschließend wurde mehrfach die Zeit gemessen, die zur Förderung von $200 \mathrm{ml}$ nötig war (Abb. 3-5).

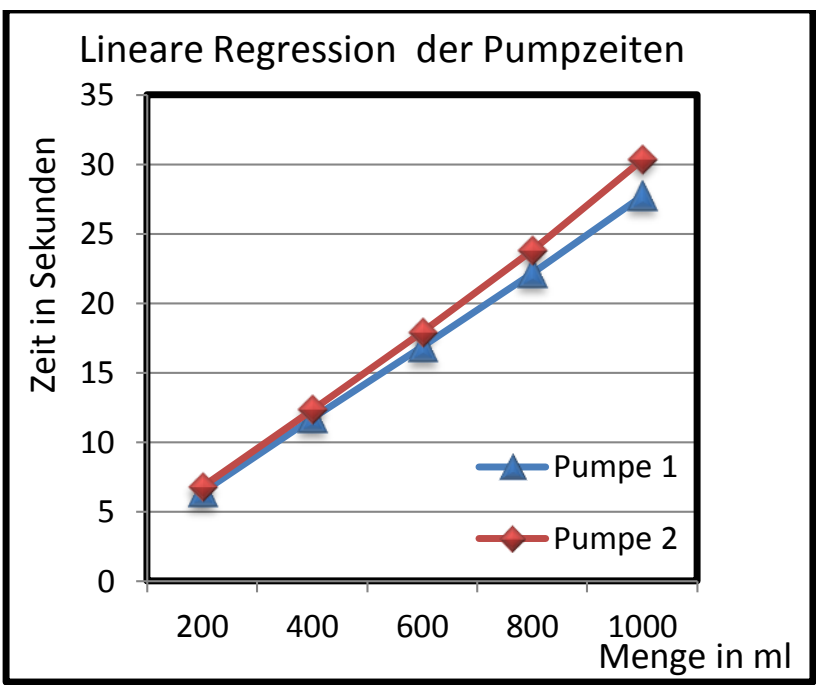

Abb. 3-4: Lineare Regression der Pumpzeiten von $200 \mathrm{ml}$ bei einer Gesamtmenge von $1000 \mathrm{ml}$.

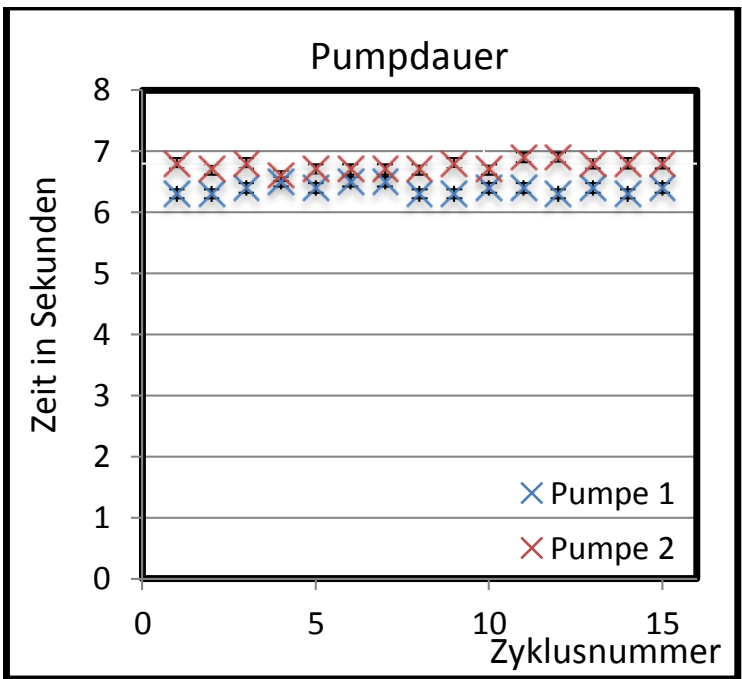

Abb. 3-5: Pumpdauer beider Pumpen für die Förderung von $200 \mathrm{ml}$ Wasser mit Standardabweichung; 15 Versuche.

Nach 15 durchgeführten Versuchen entspracht die Pumpdauer der Förderung von 200 ml Flüssigkeit für Pumpe 1 gemittelt 6,38 Sekunden, bei einer Standardabweichung von 0,07745967. Pumpe 2 benötigte für die gleiche Menge Wasser 6,76 Sekunden. Die Standardabweichung lag bei 0,04472136 .

\subsection{Probemessung - Bandscheibenmodell}

Unter Einsatz des künstlichen Bandscheibenmodells (Konstruktion siehe 2.5) erfolgten weitere Probemessungen. Zunächst wurde lediglich ein Bandscheibenpräparat simuliert, anschließend ein Segment mit Facettengelenken.

\begin{tabular}{|l|l|l|}
\hline & \multicolumn{1}{|c|}{ Ergebnis } & \multicolumn{1}{c|}{ Erwartung } \\
\hline Position der Schraubachse & zentral und ortsfest (Abb. 3-6) & zentral und ortsfest \\
\hline Ausrichtung der Drehachse & $z \approx 1$ (Abb. 3-8) & in z-Richtung (z=1) \\
\hline Schraubsteigung & $\approx 0 \mathrm{~mm} /{ }^{\circ}$ & $0 \mathrm{~mm} /{ }^{\circ}$ \\
\hline Drehwinkel-Drehmoment-Kurve & $\begin{array}{l}\text { Hookesches Verhalten mit kleiner } \\
\text { Hysterese (Abb. 3-7) }\end{array}$ & $\begin{array}{l}\text { Hookesches Verhalten mit } \\
\text { kleiner Hysterese }\end{array}$ \\
\hline
\end{tabular}

Tab. 3-2: Ergebnisse des reinen Bandscheibenmodells 
Bei den Messungen zu Axialrotation mit dem isolierten Bandscheibenmodell zeigte sich eine ortsfeste Drehachse (Abb. 3-6). Es zeigte sich eine hockey-schlägerartige Form der $\alpha(T)$-Kennlinien (Abb. 3-7). Die beobachteten Eigenschaften des Modells blieben auch nach Versatz der Vorlast nach ventral bzw. dorsal konstant. Lediglich die Ausrichtung der Achse veränderte sich bei hohen Auslenkwinkeln. In diesen Extrempunkten verkippte sie minimal nach ventral bzw. dorsal.

Der Einbau der Metallbausteine und die Interaktion selbiger bei zunehmender Auslenkung, veränderte die Bewegungsstruktur des Systems auffallend. Bei zentraler Vorlastposition zeigte die Schraubachse eine deutliche Wanderung. Zunächst im Bereich der Bandscheibe lokalisiert, verlief sie mit steigendem Rotationwinkel annähernd geradlinig in Richtung der dorsolateral platzierten Gelenke (s. Abb. 3-9). Bei Änderung der Rotationsrichtung kam es zu einer sprunghaften Verlagerung der Achsenposition um ca. $4 \mathrm{~mm}$ nach dorsal/lateral. Während der Rückbewegung erfolgte im Bereich der simulierten Facettengelenke ein Richtungswechsel der Achsenwanderung (Winkel ca. 5,5 $5^{\circ}$ und es fand sich eine anschließende geradlinige Bewegung zurück in die Bandscheibenmitte. Für eine vollständige Rotation von links nach rechts bzw. rechts nach links, ergab sich eine Migrationsstrecke von ca. $24 \mathrm{~mm}$. Die $\alpha(T)-$ Kennlinie zeigte einen hockeyschlägerartigen Verlauf, welche auf hookeschem Verhalten gründet. Im Vergleich zum bloßen Bandscheibenmodell nahm das Bewegungsausmaß um zwei Grad ab, die Neigung der Rotationsachsen zur Horizontalen mit zunehmender Auslenkung hingegen um $5^{\circ} \mathrm{zu}$. Unter Applikation einer dorsal gelegenen Vorlast waren vergleichbare Ergebnisse zu beobachten. Die Form der Rastpolkurve änderte sich nur minimal.

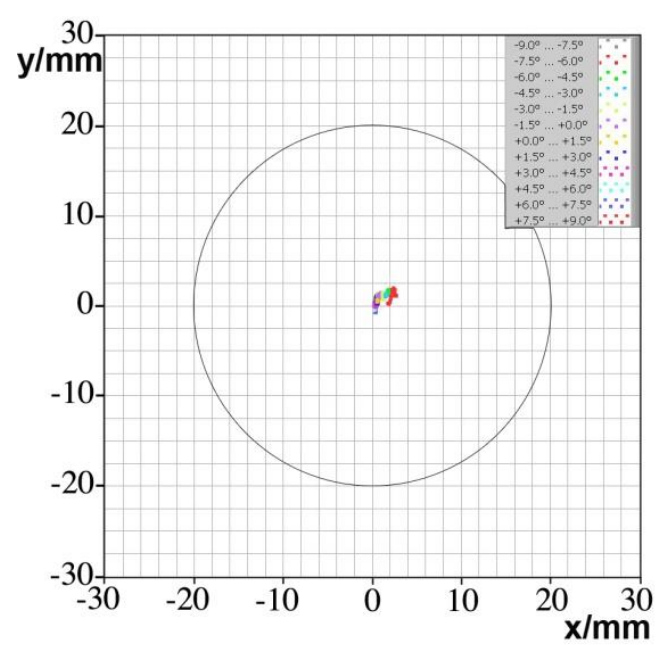

Abb. 3-6: Rastpolkurve Bandscheibenmodell ohne Gelenke; axiale Rotation, $20 \mathrm{~N}$ im WZ, ortsfeste IHA. Angedeutet: Kontur des Gummipuffers

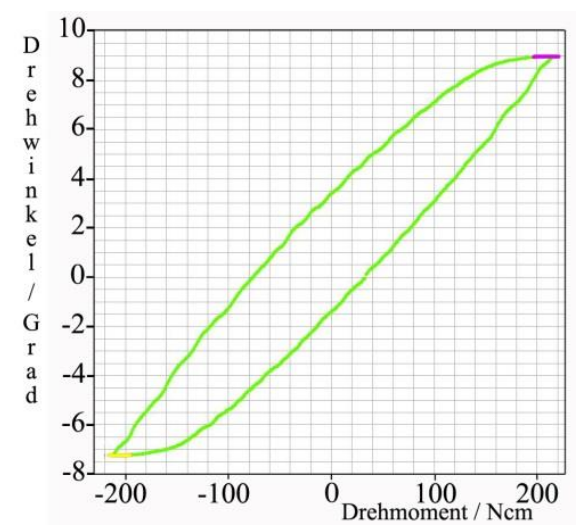

Abb. 3-7: Alpha(T)-Diagramm: Bandscheibenmodell ohne Gelenke; axiale Rotation, $20 \mathrm{~N}$ im WZ

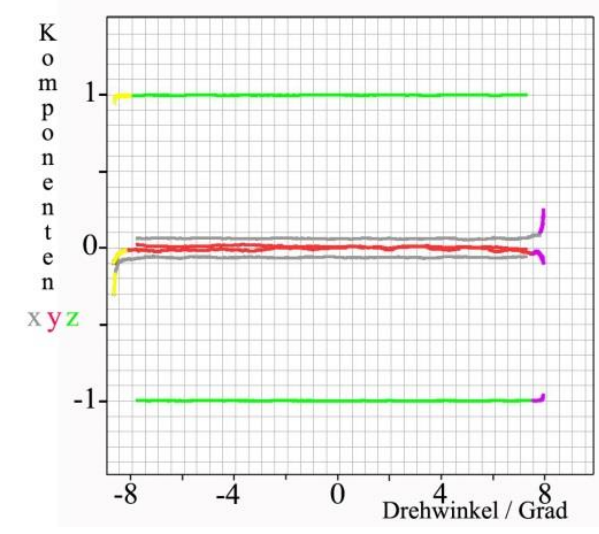

Abb. 3-8: Richtungskomponenten: Bandscheibenmodell; Eine Verkippung der Achse fand nur an den Extrempunkten der Rotation statt; axiale Rotation, $20 \mathrm{~N}$ im WZ 
Das Migrationsausmaß der momentanen Schraubachse nahm um ca. $8 \mathrm{~mm}$ zu, während das Ausmaß der Beweglichkeit erneut um zwei Grad sank. Verlagerte man die Vorlast nach ventral glich die Bewegungsstruktur der des Bandscheibenmodells: zentraler und ortsfester Verlauf der Schraubachsen, identische Achsausrichtung ( $z \approx 1)$, hookesches Verhalten, sowie gleiches Bewegungsausmaß (vgl. Abb. 3-9).

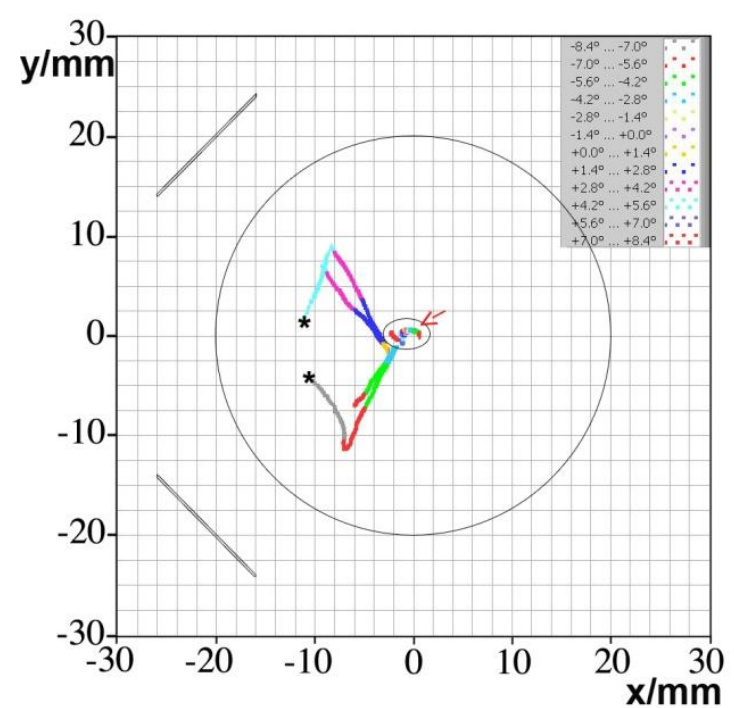

Abb. 3-9: Rastpolkurve: Bandscheibenmodell mit Gelenken; axiale Rotation, $20 \mathrm{~N}$ im WZ und $20 \mathrm{~mm}$ ventral des WZ (Pfeil). Der Startpunkt der Rastpolkurve einer Bewegungsrichtung ist jeweils mit “**، gekennzeichnet. Angedeutet: Gelenke und Gummipuffer

\subsection{Modellmessung - Prestige ${ }^{\circledR}$ LP Cervical Disc System}

\section{Ergebnisse}

Im ersten Versuch (ohne Wirbelbogengelenke) wurde eine zentrale Vorlast von $60 \mathrm{~N}$ und ein manuelles Drehmoment appliziert. Darunter zeigte sich eine Bewegung um eine nahezu ortsfeste Schraubachse (Abb. 3-10). Die Drehachse war senkrecht ausgerichtet $(z \approx 1)$, die Schraubsteigung betrug $\approx 0^{\circ} / \mathrm{mm}$. Die $\alpha(T)$-Kennlinie verlief annähernd geradlinig, ein hookesches Verhalten, wie bei den Vermessungen des Gummipuffers, war nicht erkennbar (Abb. 3-11).

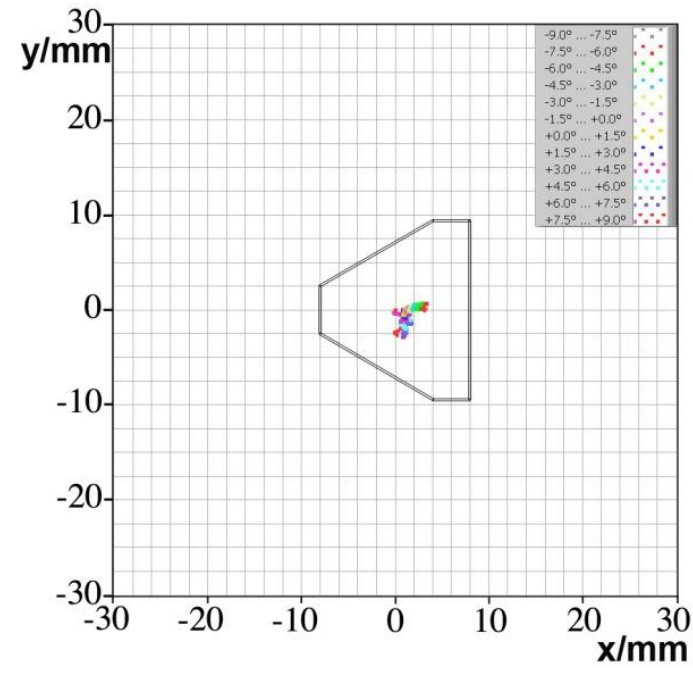

Abb. 3-10: Rastpolkurve: Prestige ${ }^{\circledR}$ LP; axiale Rotation, $60 \mathrm{~N}$ zentrale Vorlast; Die Drehachsen lagen ortsfest, zentral in der Prothese. Angedeutet: Kontur der Prestige $®$ LP

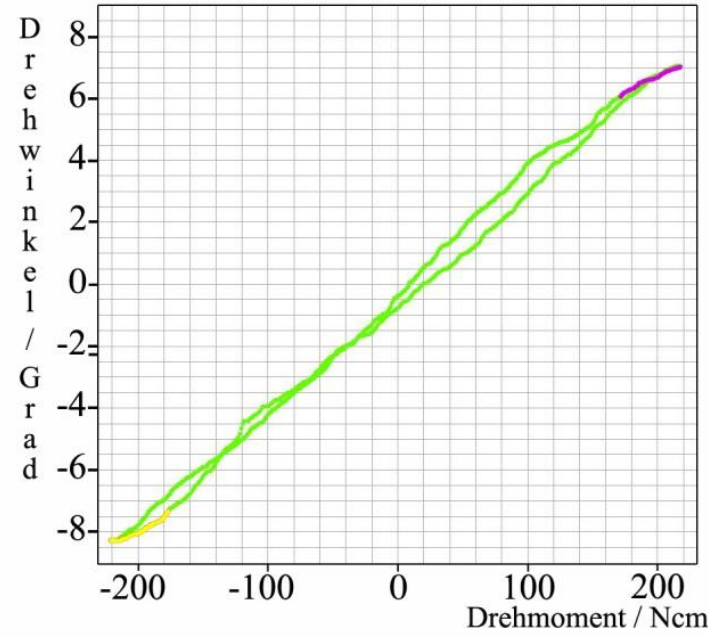

Abb. 3-11: Alpha(T)-Diagramm: Prestige ${ }^{\circledR}$ LP; Die $\alpha(\mathrm{T})$-Kennlinie verlief nahezu geradlinig, ein hookesches Verhalten war nicht erkennbar. 
Für den zweiten Versuchsteil wurden Elemente zur Simulation der Facettengelenke eingebaut und die Messungen wiederholt.

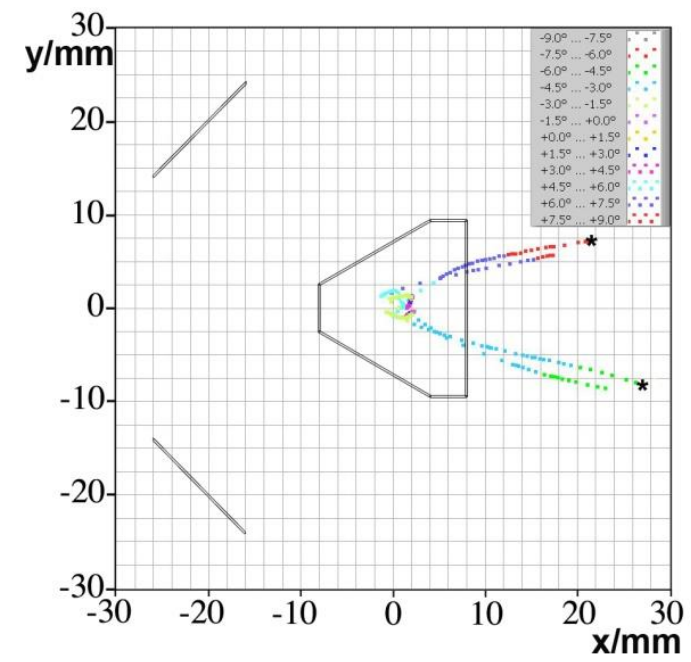

Abb. 3-12: Rastpolkurve: Prestige ${ }^{\circledR}$ LP mit Gelenkimitation; axiale Rotation, $60 \mathrm{~N}$ zentrale Vorlast; Mit zunehmender Rotation wandert die IHA nach ventrolateral. Der Startpunkt der Rastpolkurve einer Bewegungsrichtung ist jeweils mit “**، gekennzeichnet. Angedeutet: Gelenke und Prestige ${ }^{\circledR}$ LP

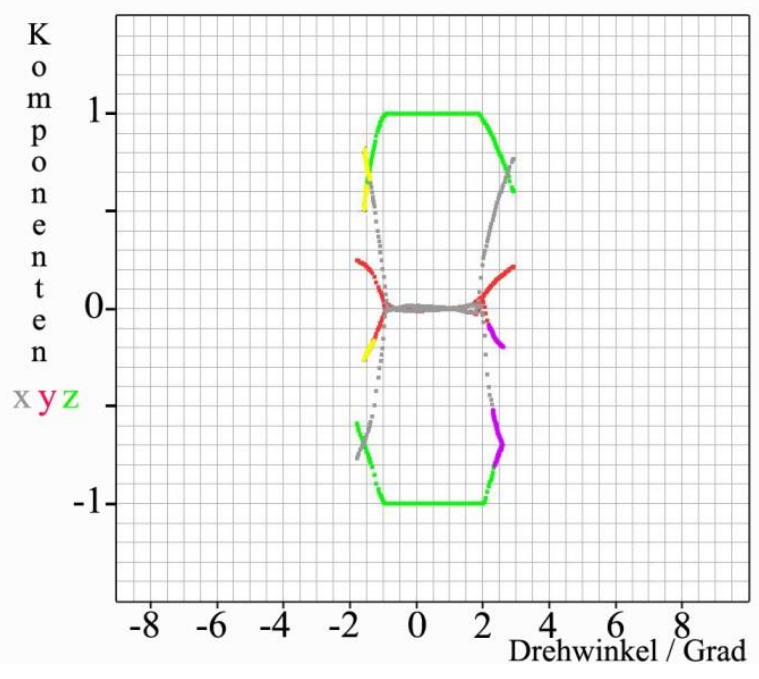

Abb. 3-13: Richtungskomponenten: Prestige® LP mit Gelenkimitation; Im Bereich hoher Rotationswinkel kam es zu einer deutlichen Verkippung der Rotationsachse, während im Winkelbereich von $-1^{\circ}$ bis $+2^{\circ}$ keinerlei Neigung vorlag.

Mit zunehmendem Rotationswinkel zeigte sich eine deutliche Migration der momentanen Schraubachse in ventrolateraler Richtung (siehe Abb. 3-12). In maximaler Rechtsdrehung lag die IHA ca. $28 \mathrm{~mm}$ ventral der zentral in der Messapparatur platzierten Prothese. Zusätzlich war sie um ca. 8 $\mathrm{mm}$ nach rechts verlagert. Im Verlauf der Linksrotation wanderte die Rotationsachse zunächst zurück in Richtung des Apparaturmittelpunktes (Sitz der Prothese). Zwischen $-3^{\circ}$ bis $6^{\circ}$ änderte sich die Position der IHA daraufhin nur geringfügig. Mit weiter ansteigendem Rotationswinkel migrierte die momentane Schraubachse nach links-ventral und endete ca. $21 \mathrm{~mm}$ ventral und ca. 7,5 mm links der Prothese. Die Bewegungsumkehr führte zu einem Versatz der IHA um ca. 4 mm nach dorsal. Von dort verlief sie im Rahmen der Rechtsdrehung, analog zur Linksdrehung, zurück in die Prothese und von dort wieder nach ventrolateral rechts, wo ihre Migration endete. Im Winkelbereich von $-1,5^{\circ}$ bis $+2^{\circ}$ zeigte sich eine zum axialen Drehmoment parallele Rotationsachse (Abb. 3-13). Ab $+2^{\circ}$ bzw. Rotationswinkeln $<-1,5^{\circ}$ fand sich eine Verkippung der Drehachse um bis zu $59^{\circ}$ in der Sagittalebene und bis ca. $29^{\circ}$ in der Horizontalebene. Die Schraubsteigung erreichte dabei einen Wert von maximal $0,1^{\circ} / \mathrm{mm}$. Der geradlinige Verlauf im $\alpha(\mathrm{T})$-Diagramm blieb indes unbeeinflusst. 


\subsection{Bestimmung des Widerstandszentrums}

Die experimentelle Bestimmung des Widerstandszentrums diente dazu, jedem Präparat einen individuellen Bezugspunkt zuzuordnen (siehe Kapitel 2.7). Die Lage des Widerstandszentrums war für die acht verwendeten Präparate nicht einheitlich, sondern variierte von Segment zu Segment. Die Positionen der Widerstandszentren bezogen auf die Mitte des Wirbelkanals (Nullpunkt der Apparatur) wurden in Tabelle 3-3 aufgeführt.

\begin{tabular}{|c|c|}
\hline Präparat & $\begin{array}{c}\text { Position des Widerstandszentrums } \\
\text { (bezogen auf die Mitte des Wirbelkanals) }\end{array}$ \\
\hline HGW 08/06 & $5 \mathrm{~mm}$ ventral \\
\hline HGW 10/06 & $5 \mathrm{~mm}$ ventral \\
\hline HGW 10/07 & $5 \mathrm{~mm}$ ventral \\
\hline HGW 11/07 & mittig im Wirbelkanal \\
\hline HGW 13/06 & $10 \mathrm{~mm}$ ventral \\
\hline HGW 14/05 & $10 \mathrm{~mm}$ ventral \\
\hline HGW 16/05 & $15 \mathrm{~mm}$ ventral \\
\hline HGW 16/07 & $10 \mathrm{~mm}$ ventral \\
\hline
\end{tabular}

Tab. 3-3: Positionen der Widerstandszentren der acht Versuchspräparate in Relation zum absoluten Nullpunkt der Apparatur.

\subsection{Axiale Rotation}

\subsubsection{Intaktes Segment}

Die Vermessung der intakten Wirbelsäulenpräparate erfolgte gemäß dem unter 2.10 („Ablauf der Messung“) gezeigten Schema. Zunächst wurde eine Vorlast im Widerstandszentrum angelegt (10 N, $20 \mathrm{~N}, 60 \mathrm{~N}$ ) bzw. entlang der sagittalen oder transversalen Achse verschoben (vergleiche Abb. 2-10). Gleichzeitig wurde ein Drehmoment von 2,25 Nm appliziert. Nach einer Einschwingphase des Gesamtsystems fand die Aufzeichnung der Tasterwerte statt, anhand welcher die biomechanischen Eigenschaften analysiert wurden. Aufgrund der Individualität der acht Versuchsobjekte und der daraus resultierenden Fülle an Daten, beschränken sich die präsentierten Ergebnisse der Übersicht halber auf konsistente Merkmale, die nicht der interindividuellen Variation unterlagen. 


\subsubsection{Lage und Wanderung der Rotationsachse}

\section{Lage}

Abbildung 3-14 zeigt exemplarisch die initiale Position der momentanen Schraubachse (IHA-Position für $\alpha=0^{\circ}$ ) in der Sagittalebene (z-x-Ebene). Zur besseren Veranschaulichung wurde die Achse in die seitliche Röntgenaufnahme des Versuchsobjektes 16/07 projiziert. In erster Linie fiel auf, dass die momentane Schraubachse (IHA) nicht parallel zum anliegenden Drehmoment $\mathrm{T}_{2}$ verlief, sondern um ca. $23^{\circ}$ nach dorsal geneigt war (siehe auch 3.6.1.2 Ausrichtung der Rotationsachse). Gleichzeitig lag sie annähernd in der Sagittalebene (seitliche Abweichung $<7^{\circ}$ ). Sie verfiel daher auf Höhe der Ableitungsebene der Messapparatur (x-Achse) im Bereich der hinteren Wirbelkörperkante ca.

$6,1 \mathrm{~mm}$ ventral des Apparaturmittelpunktes (Koordinatenursprung), während sie auf Bandscheibenhöhe annähernd mittig in der

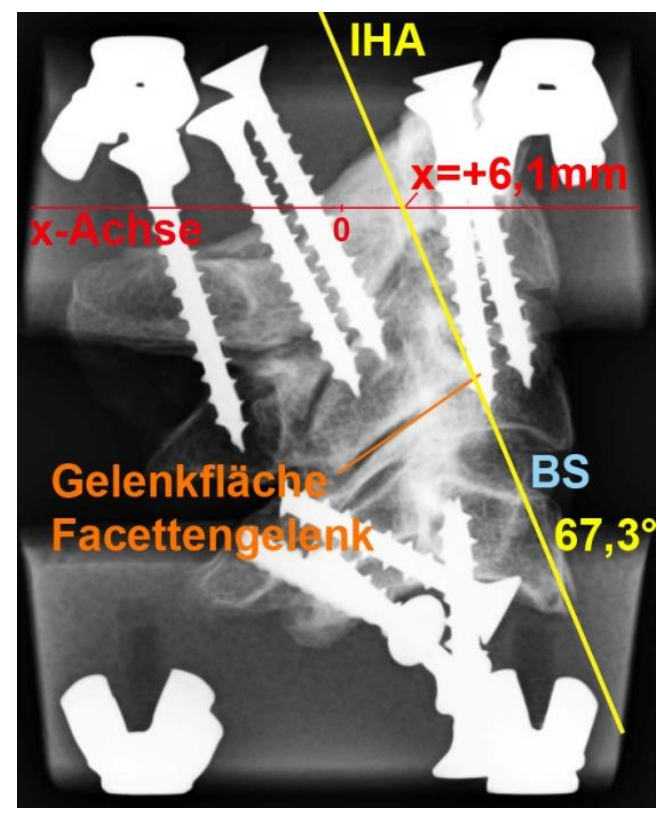

Abb. 3-14: seitliche Röntgenaufnahme des Präparates 16/07: Durchnittliche Ausrichtung der initialen Rotationsachse bei axialer Rotation. X-Achse: Ableitungsebene der Messapparatur. (Abbildung modifiziert nach Wachowski et al. 2013, Abb. 4)

Zwischenwirbelscheibe (BS) lag.

\section{Wanderung}

Die acht verwendeten Segmente zeigten trotz einer interindividuellen Variation der Wanderung der Drehachse an allen Segmenten feststellbare Merkmale. Die beschriebenen Eigenschaften waren reproduzierbar und in allen Präparaten zu finden. Exemplarisch werden die Rastpolkurven des Präparates HGW 10/07 dargestellt.

\section{Zentrale Vorlast}

Unter einer im individuellen Widerstandszentrum platzierten axialen Vorlast von $20 \mathrm{~N}$ wanderte die Rotationsachse nach Anlegen des axialen Drehmomentes $T_{2}$ von 2,25 Nm im Bereich der Bandscheibe und des vorderen Wirbelkanals. Es wurde exemplarisch eine komplette Linksrotation (Abb. 3-15) mit anschließender vollständiger Rechtsrotation (Abb. 3-16) im eingeschwungenen Zustand herausgegriffen. Die Rastpolkurve befand sich während der gesamten Bewegung hauptsächlich im Bereich der Bandscheibe, ca. 5 mm ventral des Widerstandszentrums. Zu Beginn der Linksrotation lag die momentane Schraubachse an der hinteren, linken Bandscheibenkante (Abb. 3-15, $\alpha \approx-1,6^{\circ}$; Ende des hell-grüne RPK-Abschnittes). Von dort verlief die Achse zunächst in einem ventral-konvexen 
Bogen nach vorn in die Bandscheibenmitte. Nach einer Richtungsänderung bei $0^{\circ}$ lief die Rastpolkurve wiederum bogenförmig nach dorsal aus. Der C3-Wirbel war nun maximal links rotiert $\left(\alpha \approx 1,6^{\circ}\right)$. Bei Bewegungsumkehr sprang die Drehachse ca. $6 \mathrm{~mm}$ nach rechts und ca. $8 \mathrm{~mm}$ nach dorsal in den vorderen, rechten Abschnitt des Wirbelkanals (Abb. 3-16, $\alpha \approx 1,6^{\circ}$; Ende des blauen RPKAbschnittes). Im Rahmen der Rechtsrotation wanderte die IHA anfänglich bogenförmig, dann geradlinig zurück in die Mitte der Bandscheibe und endete nach einer scharfen Wendung ( $\alpha$ zwischen $+0,8^{\circ}$ und $0^{\circ}$ ) zentral in der Zwischenwirbelscheibe. Die erneute Änderung der Rotationsrichtung mündete in einem Sprung der IHA ca. $7 \mathrm{~mm}$ nach links und $6 \mathrm{~mm}$ nach dorsal in die Ausgangsposition. Insgesamt legte die momentane Schraubachse während des kompletten Bewegungszyklus durchschnittlich eine Strecke von insgesamt 47,6 mm zurück. Im Rotationsintervall $\alpha=0,8^{\circ}$ bis $-0,8^{\circ}$ waren es ca. $18 \mathrm{~mm}$.

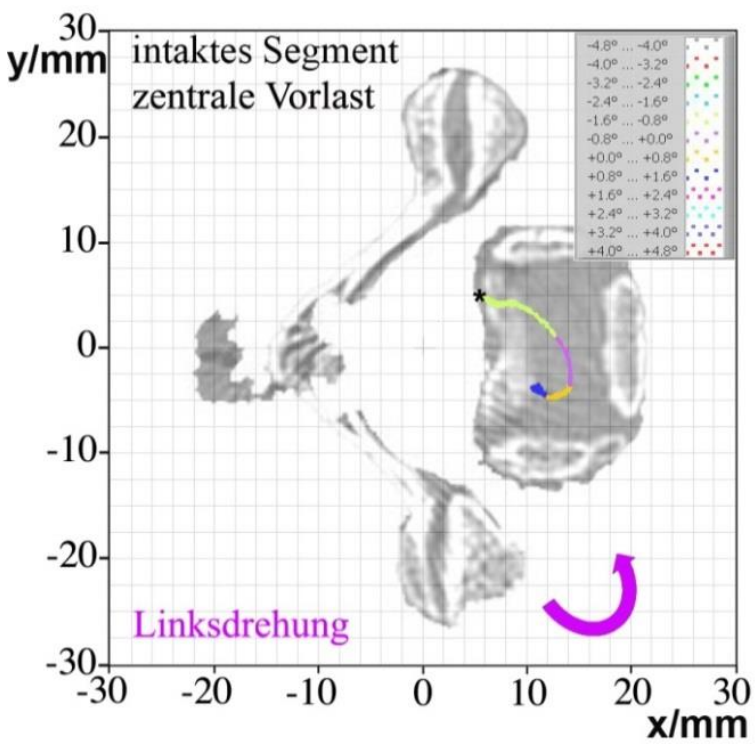

Abb. 3-15: Rastpolkurve, axiale Rotation; intaktes Segment 10/07; Linksrotation; Vorlast: $20 \mathrm{~N}$ im WZ. Die IHA wandert bogenförmig innerhalb der Bandscheibe von links (negative Rotationswinkel) nach rechts (positive Rotationswinkel). Der Startpunkt der Rastpolkurve ist mit “*“ gekennzeichnet.

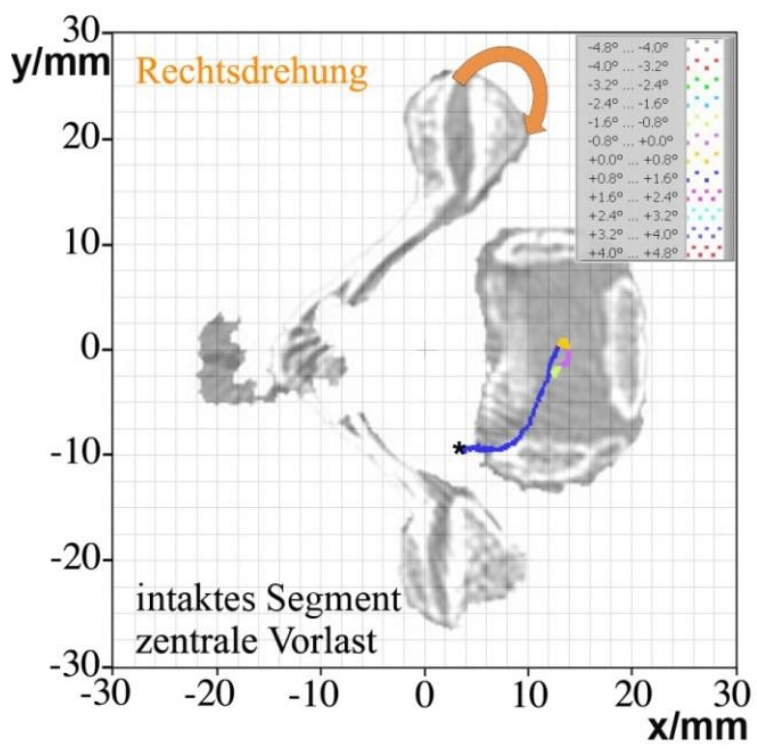

Abb. 3-16: Rastpolkurve, axiale Rotation; intaktes Segment 10/07; Rechtsrotation; Vorlast: $20 \mathrm{~N}$ im WZ. Die IHA wandert im Bereich der Bandscheibe bogenförmig von rechts (positive Rotationswinkel) nach links (negative Rotationswinkel). Der Startpunkt der Rastpolkurve ist mit “*“" gekennzeichnet.

\section{Variation des Betrages der zentralen Vorlast}

Die Erhöhung der zentralen Vorlast hatte eine leichte Stauchung der Rastpolkurve in lateraler Richtung zur Folge (s. Abb. 3-18). Die Wanderung der Schraubachsen zeigte dabei ein ähnliches Bild. Sie war lediglich auf einen etwas kleineren Bereich in der Bandscheibe beschränkt und die Migration in transversaler Richtung reduziert. Dementsprechend konnte eine Abnahme des Gesamtmigrationsausmaßes auf durchschnittlich $41,6 \mathrm{~mm}$ beobachtet werden, während im Winkelbereich zwischen $0,8^{\circ}$ und $-0,8^{\circ}$ die zurückgelegte Strecke mit $19,3 \mathrm{~mm}$ annähernd konstant blieb. 


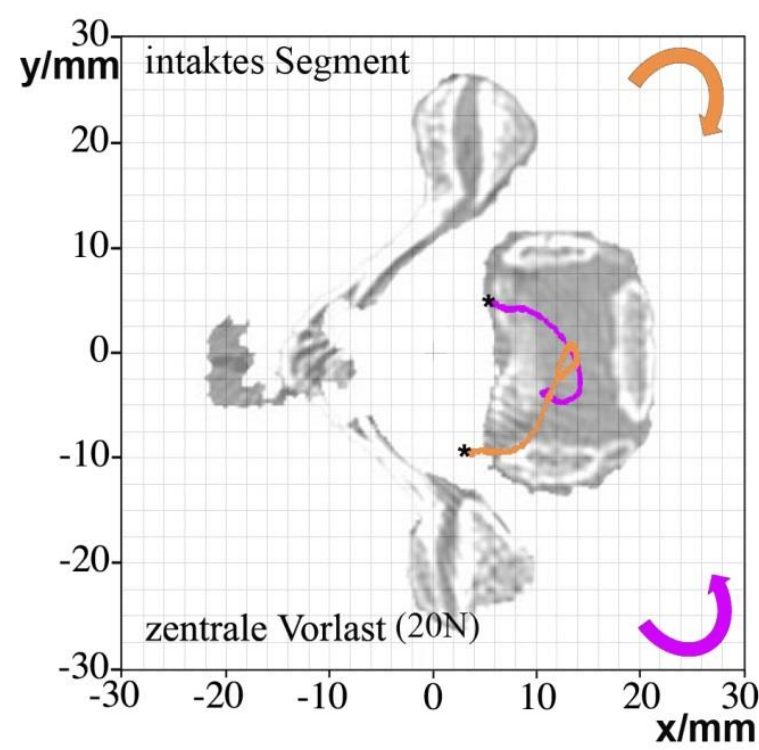

Abb. 3-17: Rastpolkurve, axiale Rotation; intaktes Segment 10/07; Vorlast: $20 \mathrm{~N}$ im WZ; violett: Linksrotation, orange: Rechtsrotation. Der Startpunkt der Rastpolkurve einer Bewegungsrichtung ist jeweils mit "**، gekennzeichnet.

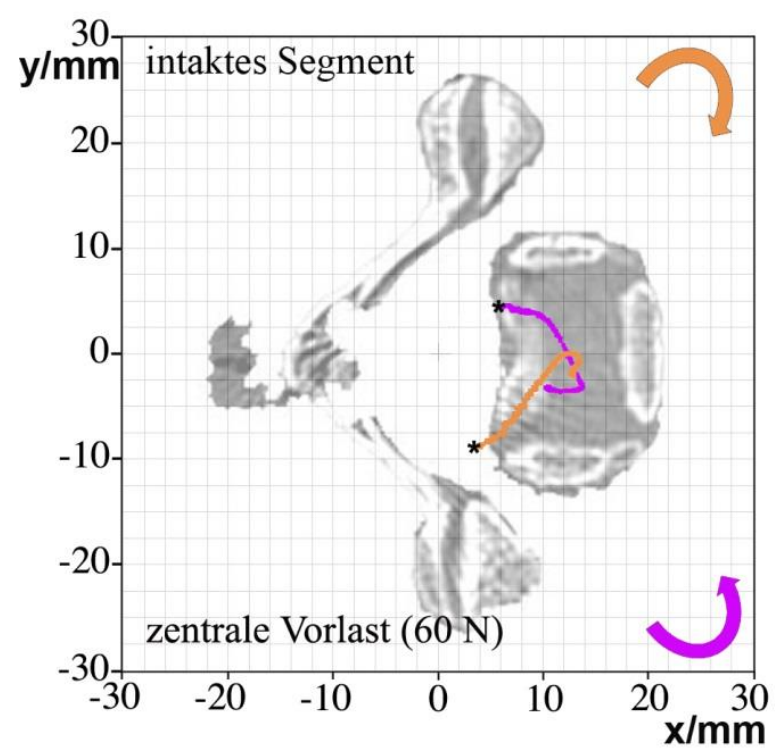

Abb. 3-18: Rastpolkurve, axiale Rotation; intaktes Segment 10/07; Vorlast: $60 \mathrm{~N}$ im WZ; violett: Linksrotation, orange: Rechtsrotation. Der Startpunkt der Rastpolkurve einer Bewegungsrichtung ist jeweils mit "**" gekennzeichnet.

\section{Sagittale Vorlastverschiebung}

Unter ventraler Belastung war eine leichte Zunahme der seitlichen Exkursionen zu beobachten (s. Abb. 3-19). Dabei verlief die Rastpolkurve während der Linksrotation nahezu gleichbleibend, endete jedoch weiter lateral im rechten, hinteren Bandscheibenbereich. Die Bewegungsumkehr ging mit einem Sprung der momentanen Rotationsachse von $4 \mathrm{~mm}$ nach rechts und $6 \mathrm{~mm}$ nach dorsal einher. Die anschließende Rechtsdrehung war mit dem Verlauf unter zentraler Vorlast $(20 \mathrm{~N})$ vergleichbar. Im Anschluss an die scharfe Wendung ( $\alpha$ zwischen $+0,8^{\circ}$ und $0^{\circ}$ ) endete sie jedoch weiter dorsal in der Bandscheibe. Der anschließende Richtungswechsel führte zu einem Sprung von $5 \mathrm{~mm}$ nach links und $6 \mathrm{~mm}$ nach dorsal in die Ausgangsposition zurück. Die durchschnittlich zurückgelegte Migrationsstrecke blieb trotz der auffälligen Veränderung der Kurvengestalt, mit 47,68 mm während eines kompletten Bewegungszyklus, nahezu konstant. Für den Winkelbereich $-0,8^{\circ}$ bis $+0,8^{\circ}$ war eine ca. 3 mm verkürzte Wanderroute zu verzeichnen (siehe Tab. 3-6). 


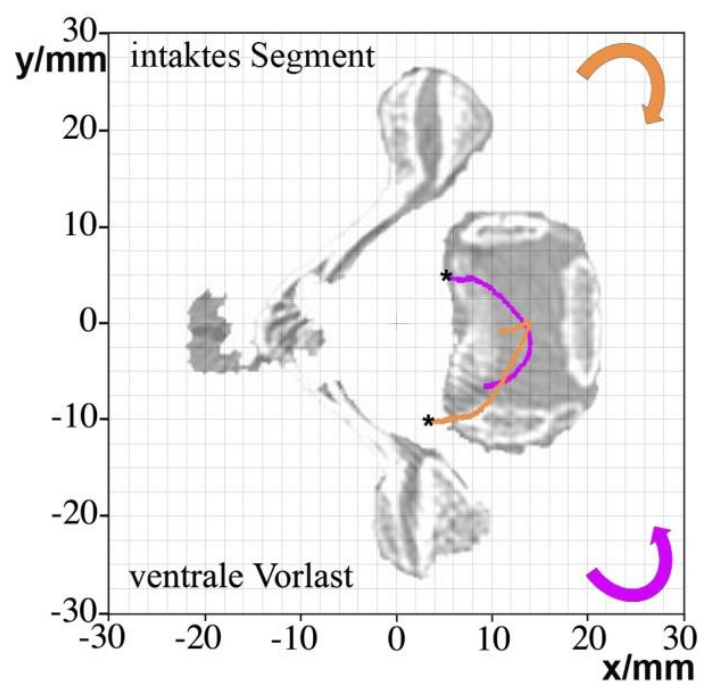

Abb. 3-19: Rastpolkurve, axiale Rotation; intaktes Segment 10/07; Vorlast: 20 N 20 mm ventral des WZ; violett: Linksrotation, orange: Rechtsrotation. Der Startpunkt der Rastpolkurve einer Bewegungsrichtung ist jeweils mit "*“ gekennzeichnet.

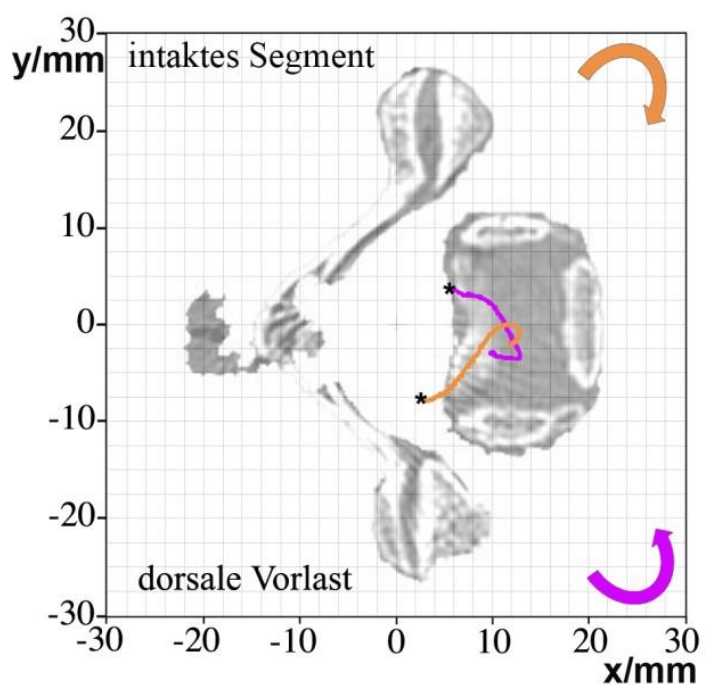

Abb. 3-20: Rastpolkurve, axiale Rotation; intaktes Segment 10/07; Vorlast: 20 N 20 mm dorsal des WZ; violett: Linksrotation, orange: Rechtsrotation. Der Startpunkt der Rastpolkurve einer Bewegungsrichtung ist jeweils mit “*“" gekennzeichnet.

Wurde die axiale Vorlast dorsal des Widerstandszentrums angebracht, zeigte sich eine Zentralisation der wandernden Schraubachse, ähnlich der bei erhöhter zentraler Vorlast (Abb. 3-20, vergleiche Abb. 3-18). Dabei kam es zu einem Anstieg des Migrationsausmaßes zwischen $\alpha=0,8^{\circ}$ und $-0,8^{\circ}$ um ca. 5 $\mathrm{mm}$ auf ca. $23 \mathrm{~mm}$, während die insgesamt zurückgelegte Strecke mit durchschnittlich $39 \mathrm{~mm}$ deutlich kürzer war. Zusätzlich kam es zu einer Verlagerung der Rastpolkurve nach dorsal (Abb. 3-20). Für Links-, wie auch für Rechtsrotation beschrieb die im hinteren Bandscheibenfach wandernde IHA erneut eine bogenförmige Form.

Die Wendepunkte im Migrationsverlauf $\left(\alpha \approx 0^{\circ}\right)$ waren während der Rechtsdrehung im Allgemeinen deutlich schärfer, als bei der Linksdrehung.

\section{Laterale Vorlastverschiebung}

Durch eine Vorlastapplikation lateral des Widerstandszentrums kam es zu einer Verschiebung der Rastpolkurve zur jeweils belasteten Seite (Abb. 3-21). Die momentane Schraubachse lag dabei aber weiterhin im Bereich der Bandscheibe. Die Gestalt der Rastpolkurve blieb mit dem Verlauf unter zentraler Vorlast vergleichbar, jedoch wurde sie in Richtung der belasteten Seite verzogen.

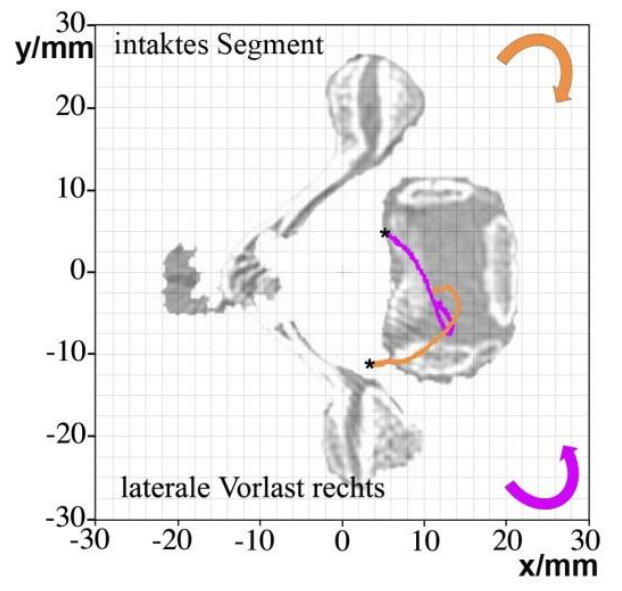

Abb. 3-21: Rastpolkurve, axiale Rotation; intaktes Segment 10/07; Vorlast: 20 N 15 $\mathrm{mm}$ lateral-rechts des WZ; violett: Linksrotation, orange: Rechtsrotation. Der Startpunkt ist mit “**" gekennzeichnet. 


\subsubsection{Ausrichtung der Rotationsachse}

Eine genaue Kenntnis über die dreidimensionale Ausrichtung und Lage der IHA ist für die Beurteilung der Rastpolkurve und deren Gestalt von grundlegender Bedeutung. Abbildung 3-22 zeigt die Ausrichtung der momentanen Schraubachse des Präparates 10/07 während eines Bewegungszyklus. In erster Linie fiel die dorso-ventrale Neigung der momentanen Schraubachse auf (vergleiche 3.6.1.1 „Lage und Wanderung der Rotationsachse“). Der durchschnittliche Neigungswinkel betrug ca. $-67^{\circ}$, was einer dorsalen Verkippung der momentanen Schraubachse in Relation zum axial ausgerichteten Drehmoment von $23^{\circ}$ entsprach (IHA nicht parallel zu $T_{z}$; siehe Abb. 3-22 und Abb. 3-14). Jedoch lag sie initial beinahe in der Sagittalebene (seitliche Abweichung $<7^{\circ}$ ). Grundsätzlich änderte sich die Ausrichtung der Rotationsachse mit zunehmendem Drehwinkel nur geringfügig, was anhand der Richtungskomponenten des Einheitsvektors (Abb. 3-23) erkennbar war.

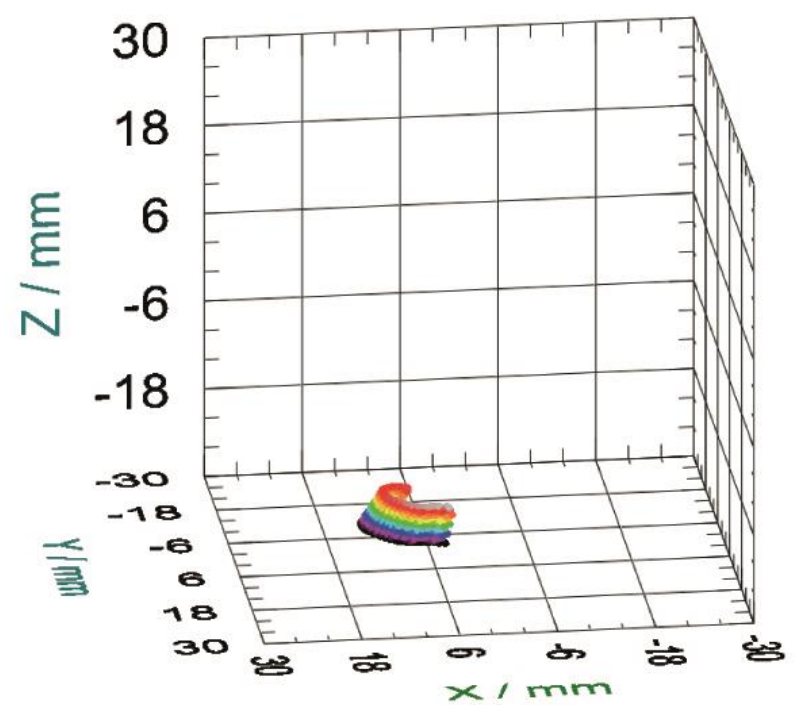

Abb. 3-22: 3D-Rekonstruktion Rastpolkurve, axiale Rotation; 10/07 intakt, Vorlast: $20 \mathrm{~N}$ im WZ. Die Dorsalneigung der IHA (ca. 23) war während des gesamten Bewegungszyklus annähernd konstant.

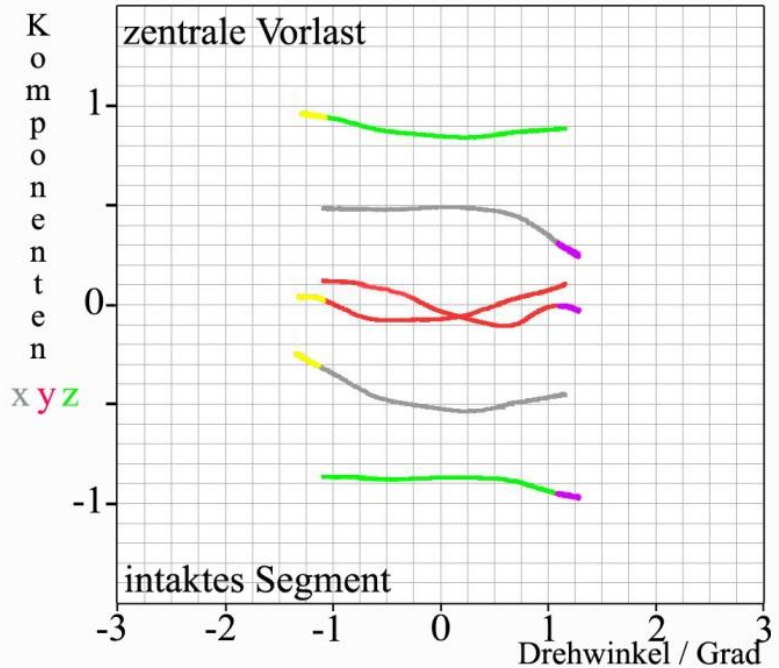

Abb. 3-23: Richtungskomponenten der Schraubachse: axiale Rotation, Vorlast: $20 \mathrm{~N}$ im WZ

Die y-Komponenten $\left(e_{y}\right)$ des Richtungsvektors e $(\alpha)$ der momentanen Schraubachse blieben in allen Rotationsbereichen klein. Anhand der Änderung der $x$ - und z-Komponente $\left(e_{x} ; e_{z}\right)$ ließ sich eine zusätzliche Verkippung der IHA um durchschnittlich ca. $10^{\circ}$ auf insgesamt ca. $33^{\circ}$ in Richtung der Horizontalebene (Neigung nach dorsal) berechnen. Die Erhöhung bzw. Verlagerung der Vorlast zeigte nahezu keinen Effekt auf die initiale Ausrichtung der IHA (Tab. 3-7). 


\subsubsection{Drehwinkel-Drehmoment-Kennlinie, Bewegungsausmaß und Steifigkeit}

Die Betrachtung der Alpha(T)-Kennlinie der Versuchsobjekte zeigte eine für alle Präparate geltende Konstellation. Das applizierte Drehmoment $T_{z}$ von maximal 2,25 Nm blieb während der Versuche konstant. Im Vergleich zum Bandscheibenmodell lag sowohl bei den intakten Segmenten, als auch nach Prothesenimplantation, eine sigmoide Verlaufsform der Drehmoment-Drehwinkel-Kurve vor. Der Anstieg des Drehwinkels $\alpha$ nahm nahe dem Erreichen des maximalen Bewegungsumfanges (ROM; „Range of motion“) des Segmentes trotz eines weiterhin linear ansteigenden Drehmoments $T_{z}(t)$ nicht mehr geradlinig zu. Gleichzeitig ließ sich mit zunehmendem Rotationswinkel eine deutliche Progression der differentiellen Steifigkeit der Segmente beobachten. Die Graphen der Hin- und Rückbewegung verliefen asymmetrisch und zeigten mitunter eine deutliche Hysterese.

\section{Zentrale Vorlast}

Die Analyse der Drehwinkel-Drehmoment-Kennlinie und des Steifigkeitsdiagrammes erfolgte wie beschrieben unter einer anfänglichen, zentralen Vorlast von $20 \mathrm{~N}$, sowie nach schrittweiser Erhöhung auf $30 \mathrm{~N}$ und $60 \mathrm{~N}$. Dabei ließ sich mit zunehmender Belastung ein deutlicher Anstieg der Segmentsteifigkeit verzeichnen. Die minimale Steifigkeit (Rotationswinkel $\alpha=0^{\circ}$ ) nahm um durchschnittlich 43\% zu (60 N Vorlast, vgl. Tabelle 3-4). Dabei war eine Abnahme des Bewegungsausmaßes (ROM) zu beobachten (ca. 11,67\%).

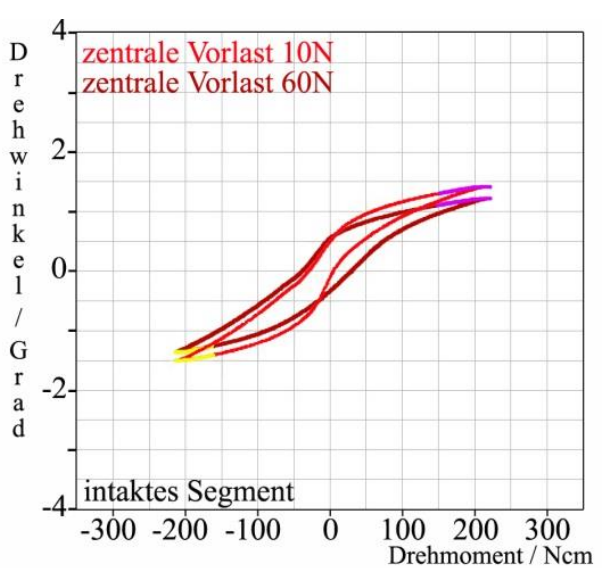

Abb. 3-24: Alpha(T)-Diagramm, axiale Rotation; 10/07 intakt, Vorlast: 20 und $60 \mathrm{~N}$ im WZ

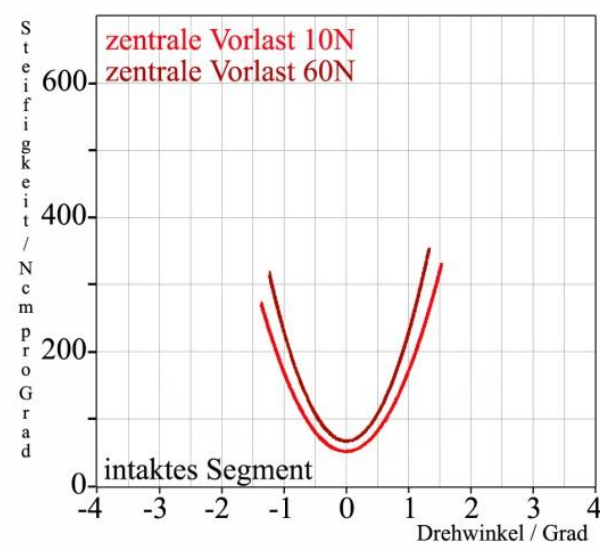

Abb. 3-25: Steifigkeitsdiagramm, axiale Rotation; 10/07 intakt, Vorlast: 20 und $60 \mathrm{~N}$ im WZ

\begin{tabular}{|c|c|c|c|c|}
\hline Intaktes Segment & $\begin{array}{l}\text { Steifigkeit } \\
\text { [Ncm/deg] }\end{array}$ & $\begin{array}{l}\text { Steifigkeit } \\
\text { [Ncm/deg] } \\
\text { rel [\%] }\end{array}$ & ROM [deg] & $\begin{array}{l}\text { ROM [deg] rel } \\
\text { [\%] }\end{array}$ \\
\hline $20 \mathrm{~N}$ zentral & $43,75(\sigma=9,48)$ & 100 & $4,28(\sigma=1,54)$ & 100 \\
\hline $60 \mathrm{~N}$ zentral & $62,63(\sigma=28,27)$ & $143(\sigma=40)$ & $3,85(\sigma=1,59)$ & $88,33(\sigma=8,06)$ \\
\hline
\end{tabular}

Tab. 3-4: Durchschnittliche Steifigkeit (Ncm/deg) und Range of motion (ROM) aller intakten

Bewegungssegmente bei verschiedenen Vorlastbeträgen im Widerstandszentrum; axiale Rotation, jeweilige

Standardabweichung $(\sigma)$ in Klammern 
Die Verschiebung der konstanten Vorlast (20 N) entlang der Sagittalachse führte bei ventraler Platzierung zu einer Flexion des Messobjekts, die dorsale Vorbelastung resultierte in einer Extension. Dabei zeigten die Messungen eine gemittelte Zunahme des ROM bei ventraler Vorlastposition von ca. 13\% im Vergleich zur zentralen Vorlast. Analog verringerte sich die minimale Steifigkeit um 27\% (s. Tabelle 3-5). In Extensionsstellung war hingegen ein Anstieg der minimalen Steifigkeit zu beobachten. Dieser betrug bei einer Vorlastapplikation $20 \mathrm{~mm}$ dorsal des Widerstandzentrums durchschnittlich 50\%. Das Bewegungsausmaß nahm dabei um rund $17 \%$ ab.

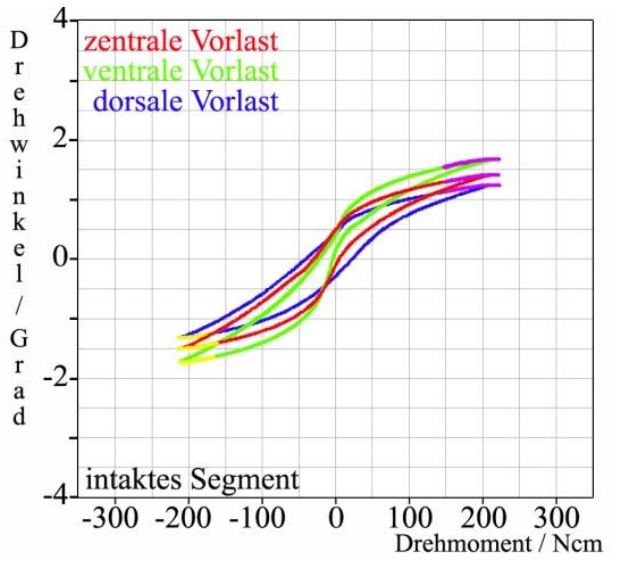

Abb. 3-26: Alpha(T)-Diagramm, axiale Rotation; 10/07 intakt, Vorlast: 20 N 20 mm ventral, dorsal und im WZ

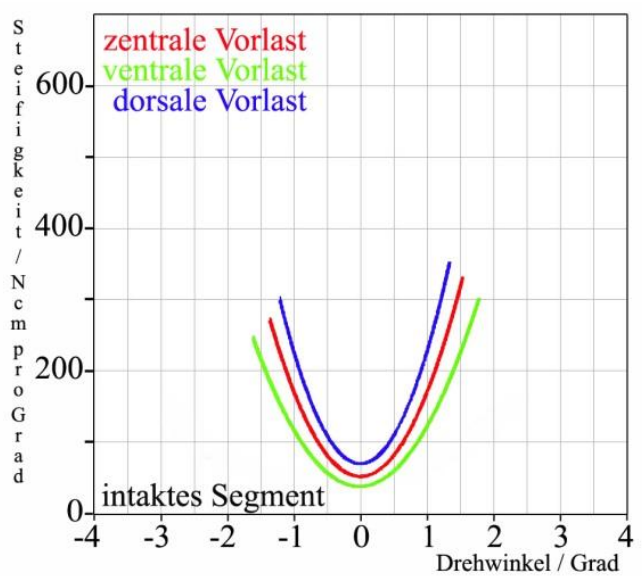

Abb. 3-27: Steifigkeitsdiagramm, axiale Rotation; 10/07 intakt, Vorlast: 20 N 20 mm ventral, dorsal und im WZ

\section{$20 \mathrm{~N} 20 \mathrm{~mm}$ ventral}

\begin{tabular}{|llll|}
\hline Intaktes Segment & $20 \mathrm{~N}$ zentral & $20 \mathrm{~N} 20 \mathrm{~mm}$ ventral & 20 N $20 \mathrm{~mm}$ dorsal \\
\hline$[\mathrm{Ncm} / \mathrm{deg}]$ & $42,75(\sigma=9,48)$ & $30,38(\sigma=7,05)$ & $\mathbf{6 6}(\sigma=26,99)$ \\
\hline$[\mathrm{Ncm} / \mathrm{deg}]$ rel [\%] & 100 & $73(\sigma=17)$ & $\mathbf{1 5 0}(\sigma=42)$ \\
\hline & & & \\
\hline ROM [deg] & $4,28(\sigma=1,54)$ & $4,74(\sigma=1,5)$ & $\mathbf{3 , 5 6}(\sigma=1,53)$ \\
\hline ROM rel [\%] & 100 & $112,86(\sigma=9,04)$ & $\mathbf{8 3 , 1 8}(\sigma=11)$ \\
\hline
\end{tabular}

Tab. 3-5: Steifigkeit $(\mathrm{Ncm} /[\mathrm{deg}])$ und Range of motion (ROM) der intakten Bewegungssegmente bei verschiedenen Vorlastpositionen; axiale Rotation, jeweilige Standardabweichung $(\sigma)$ in Klammern. Die ventrale Positionierung der VL ging mit einem Steifigkeitsabfall einher, welcher in einer Zunahme des Bewegungsausmaßes resultierte. Bei dorsaler Vorlastposition zeigte sich hingegen eine Zunahme der Steifigkeit mit reduziertem Bewegungsausmaß.

\section{Laterale Vorlastverschiebung}

Die Verlagerung der Vorlast in transversaler Richtung hatte keine erwähnenswerte Konsequenz. Sowohl die minimale Steifigkeit (initial ca. $46 \mathrm{Ncm} / \mathrm{deg}$ ), als auch das Bewegungsausmaß (ca. 5\% Abweichung) der untersuchten Segmente waren mit den Ergebnissen der zentralen Belastung vergleichbar. 


\subsubsection{Bandscheibenprothesen Prestige ${ }^{\circledast}$ LP Cervical Disc System \& Bryan ${ }^{\circledast}$ Cervical Disc System}

\subsubsection{Lage und Wanderung der Rotationsachse}

Die deutliche Wanderung der momentanen Rotationsachse, wie sie beim intakten Segment zu beobachten war, blieb nach der Implantation der beiden Bandscheibenprothesen erhalten. Die Abbildungen 3-28 bis 3-30 zeigen die Gestalten der Rastpolkurve des Präparates 10/07 im physiologischen Zustand und nach Prothesenimplantation. Obwohl die Kurvengestalt und die Lage nach der Implantation sichtbar von den Ergebnissen unter physiologischen Bedingungen abwichen, bestand eine zum intakten Segment gleichbleibende Tendenz des Migrationsverhaltens in Abhängigkeit von der Vorlastposition. So waren der Dorsalversatz und die transversale Stauchung der RPK bei dorsaler Vorlastposition auch nach der Implantation der Bandscheibenprothesen (besonders bei $\operatorname{Bryan}^{\circledR}$ ) erkennbar. Nach der Implantation der Prestige LP $^{\circledR}$ war bei dorsaler Platzierung der axialen Vorlast ein Anstieg des Migrationsumfanges um durchschnittlich ca. $15 \mathrm{~mm}$ für den gesamten Zyklus und annähernd $6 \mathrm{~mm}$ für Rotationswinkel zwischen $-0,8^{\circ}$ und $+0,8^{\circ}$ (Tabelle 3-6) erkennbar. Unter ventraler Vorlast zeigte sich im Winkelintervall $0,8^{\circ}$ bis $-0,8^{\circ}$ beim intakten Segment, wie auch nach Implantation der Bandscheibenprothese, ein verringertes Wanderungsausmaß. Die Verschiebung in transversaler Richtung zeigte hingegen keine Signifikanz.

Infolge des Bandscheibenersatzes durch Bryan ${ }^{\circledR}$ Cervical Disc stieg das Gesamtausmaß der Wanderung im Vergleich zum physiologischen Zustand unter zentraler und dorsaler Belastung stark an (Tab. 3-6). Eine Belastung ventral des Widerstandszentrums änderte die zurückgelegte Migrationsstrecke kaum. In Abhängigkeit der Vorlastposition zeigte sich im Winkelintervall $0,8^{\circ}$ bis $-0,8^{\circ}$ nach der Implantation der Bryan ${ }^{\circledR}$ Cervical Disc die gleiche Tendenz im Migrationsverhalten, wie beim intakten Präparat. In Flexionsstellung sank das Ausmaß der IHA-Wanderung zwischen $0,8^{\circ}$ und $-0,8^{\circ}$, in Extensionsstellung ließ sich hingegen ein deutlicher Anstieg der selbigen verzeichnen.

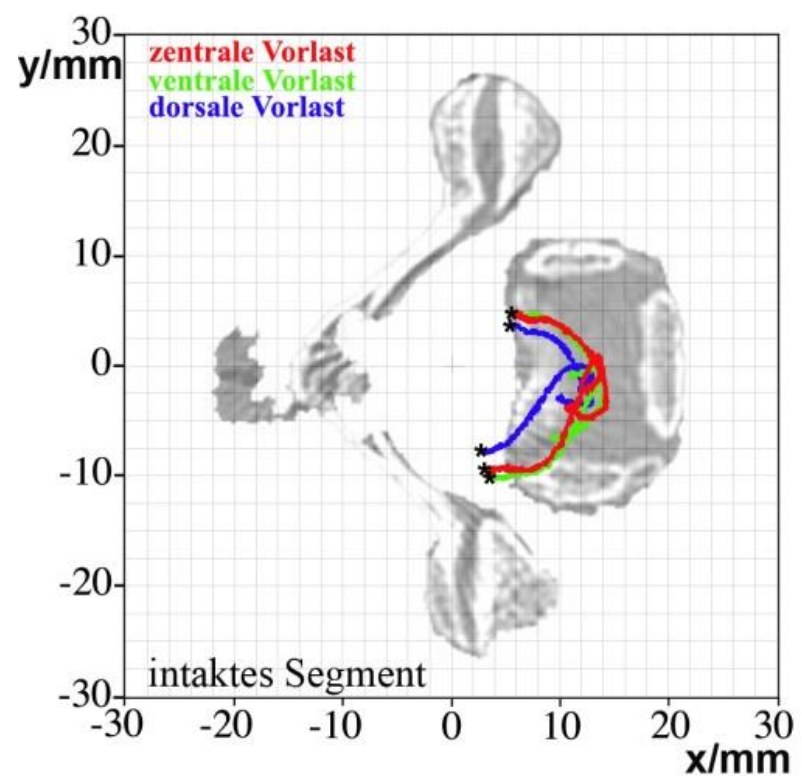

Abb. 3-28: Rastpolkurve, axiale Rotation; intaktes Segment 10/07; Vorlast: 20 N 20 mm dorsal bzw. ventral und im WZ. Der Migrationsverlauf änderte sich bei unterschiedlichen Vorlastpositionen nur geringfügig. Dorsal platzierte Vorlasten führten zu einem leichten Versatz der IHA nach dorsal. Der Startpunkt der Rastpolkurve einer Bewegungsrichtung ist jeweils mit “*“" gekennzeichnet. 


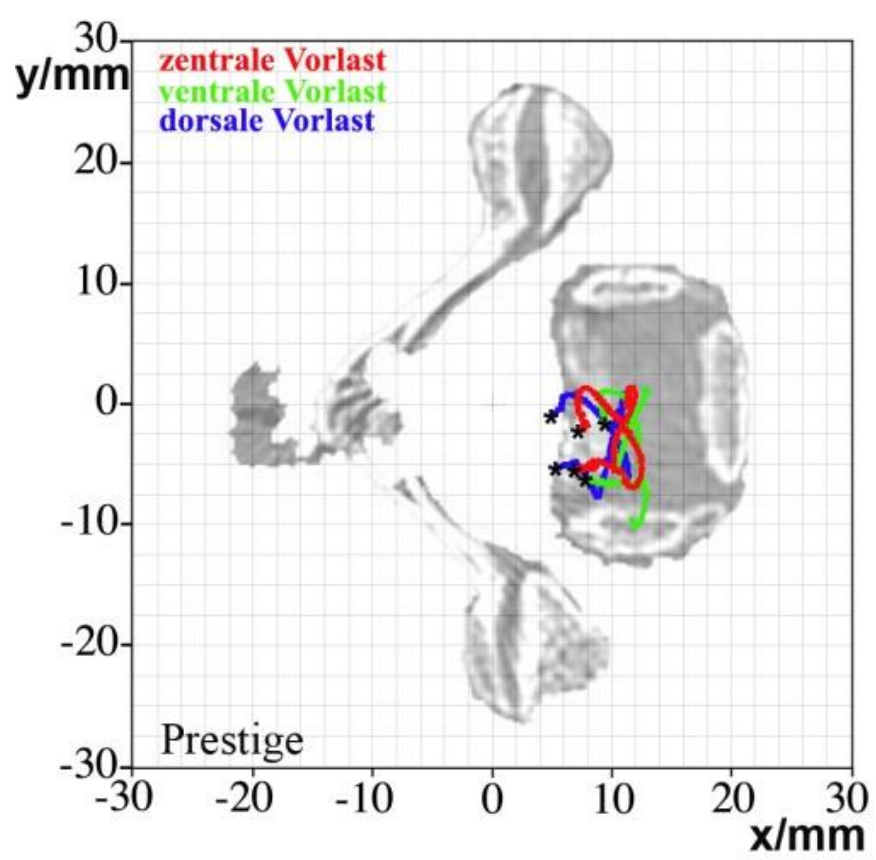

Abb. 3-29: Rastpolkurve, axiale Rotation; Prestige $\AA$ LP Cervical Disc 10/07; Vorlast: 20 N $20 \mathrm{~mm}$ dorsal bzw. ventral und im WZ. Die Gestalt änderte sich im Vergleich zum intakten Segment (transversal gestaucht). Die vorlastabhängige Änderung des Migrationsverlaufes blieb tendenziell gleich (leichter Dorsalversatz der IHA bei dorsaler Vorlastposition). Der Startpunkt der Rastpolkurve einer Bewegungsrichtung ist jeweils mit “**، gekennzeichnet.

Besonders auffällig war der konstante Versatz der momentanen Schraubachse in dorsaler Richtung nach TDA. Für $\alpha=0^{\circ}$ verlagerte sich die IHA unter Prestige $L P^{\circledR}$ durchschnittlich um $\Delta x=-5,9 m m$, bei einer Standardabweichung von $4,99 \mathrm{~mm}(\mathrm{p}<1 \%)$ und um $\Delta y=-0,42 \mathrm{~mm}$ (Lateralversatz) mit einer Standardabweichung von 4,64 mm. Die durchschnittliche Positionsänderung der Drehachse mit Bryan ${ }^{\circledR}$ Cervical Disc betrug: $\Delta x=$ $-8,15 \mathrm{~mm}(\mathrm{SD}=3,93 \mathrm{~mm})$ und $\Delta \mathrm{y}=-1,87 \mathrm{~mm}$ bei einer Standardabweichung von 4,08 $\mathrm{mm}$. Dieser Schraubachsversatz wird unter 3.6.2.2 "Ausrichtung der Rotationsachse nach Prothesenimplantation" genauer betrachtet.

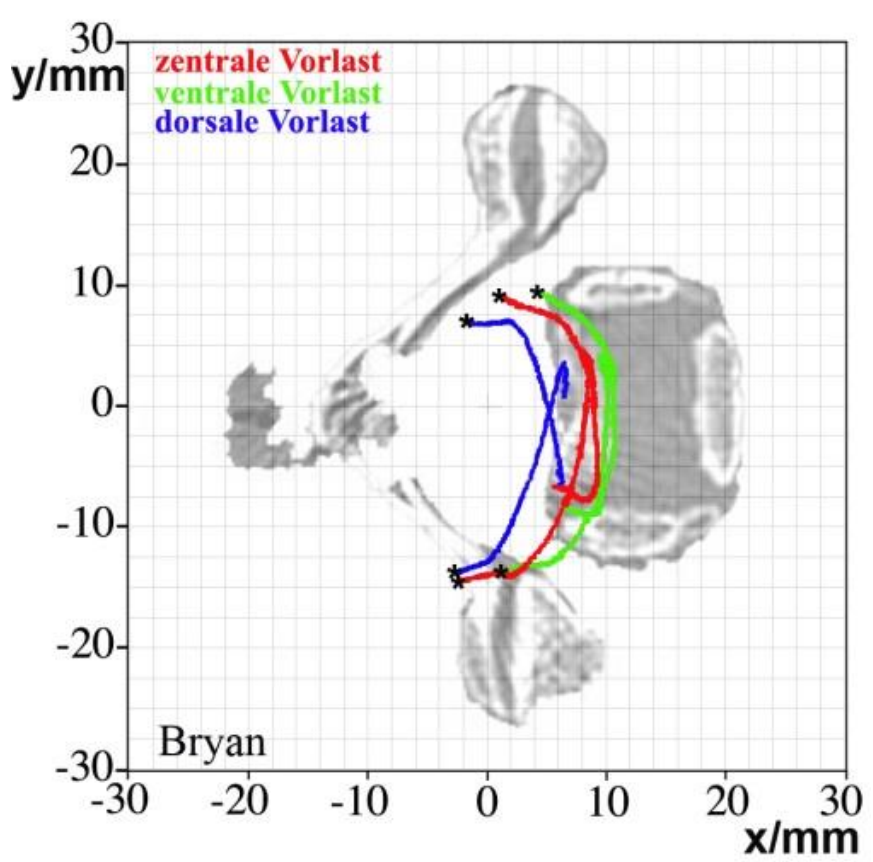

Abb. 3-30: Rastpolkurve, axiale Rotation; Bryan ${ }^{\circledR}$ Cervical Disc 10/07; Vorlast: 20 N 20 mm dorsal bzw. ventral und im WZ. Die Gestalt änderte sich im Vergleich zum intakten Segment (Zunahme der seitlichen Exkursion). Die vorlastabhängige Änderung des Migrationsverlaufes blieb hingegen tendenziell gleich (Dorsalversatz der IHA bei dorsaler Vorlastposition). Der Startpunkt der Rastpolkurve einer Bewegungsrichtung ist jeweils mit “*“ gekennzeichnet.

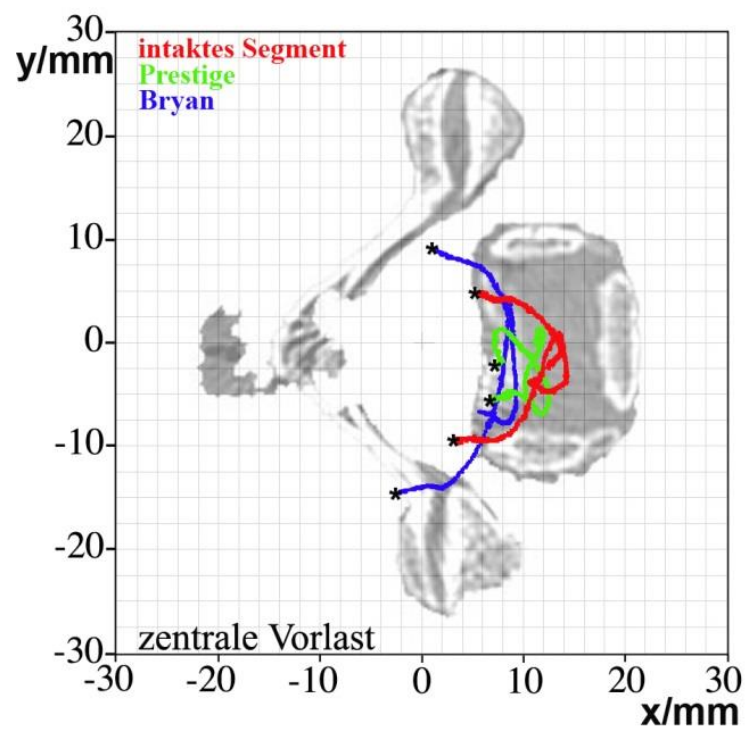

Abb. 3-31: Rastpolkurve, axiale Rotation; Präparat 10/07; Vorlast: $20 \mathrm{~N}$ im WZ. Nach Implantation der Prothesen zeigten sich eine Änderung der Kurvengestalt, sowie ein Versatz der IHA nach dorsal. Der Startpunkt der Rastpolkurve einer Bewegungsrichtung ist jeweils mit “**، gekennzeichnet. 


\begin{tabular}{|c|c|c|c|}
\hline & $20 \mathrm{~N}$ im WZ & $\begin{array}{c}20 \mathrm{~N} 20 \mathrm{~mm} \\
\text { ventral }\end{array}$ & $\begin{array}{c}20 \text { N } 20 \text { mm } \\
\text { dorsal }\end{array}$ \\
\hline \multicolumn{4}{|l|}{ Intaktes Segment } \\
\hline Migrationsstrecke gesamt & $47,6(\sigma=19,2)$ & $47,68(\sigma=13,84)$ & $39,01(\sigma=11,55)$ \\
\hline Intervall $0,8^{\circ}$ bis $-0,8^{\circ}$ & $18,03(\sigma=11,85)$ & $15,33(\sigma=7)$ & $22,99(\sigma=13,97)$ \\
\hline \multicolumn{4}{|c|}{ Prestige $^{\circledR}$ LP Cervical Disc System } \\
\hline Migrationsstrecke gesamt & $46,35(\sigma=23,5)$ & $46,89(\sigma=14,49)$ & $54,55(\sigma=28,35)$ \\
\hline Intervall $0,8^{\circ}$ bis $-0,8^{\circ}$ & $16,94(\sigma=14,33)$ & $14,28(\sigma=7,64)$ & $28,77(\sigma=23,96)$ \\
\hline \multicolumn{4}{|l|}{ Bryan $^{\circledR}$ Cervical Disc System } \\
\hline Migrationsstrecke gesamt & $60,66(\sigma=26,77)$ & $45(\sigma=16,15)$ & $80,13(\sigma=3,72)$ \\
\hline Intervall $0,8^{\circ}$ bis $-0,8^{\circ}$ & $17,7(\sigma=9,04)$ & $9,88(\sigma=23,14)$ & $33(\sigma=19,44)$ \\
\hline
\end{tabular}

Tab. 3-6: Durchschnittliches Migrationsausmaß der IHA der Intakten Segmente und nach Implantation der Prestige ${ }^{\circledR}$ LP Cervical Disc System bzw. Bryan ${ }^{\circledR}$ Cervical Disc System. Darstellung der Werte für einen vollständig abgelaufenen Bewegungszyklus und das Rotationsintervall $0,8^{\circ}$ bis $-0,8^{\circ}$. Angaben in Millimeter, jeweilige Standardabweichung $(\sigma)$ in Klammern. Im Rotationsintervall $0,8^{\circ}$ bis $-0,8^{\circ}$ zeigte sich eine gleichbleibende Tendenz des Migrationsausmaßes in Abhängigkeit der Vorlastposition. Mit der Prothesenimplantation war im Gegensatz zum intakten Segment eine erhöhte Gesamtmigration unter dorsaler Vorlast zu beobachten. Unter Bryan® war zudem eine größere Migrationsrate bei Belastung im WZ zu sehen. Unter ventraler VL blieb das Migrationsausmaß vor und nach Prothesenimplantation annähernd gleich.

\subsubsection{Ausrichtung der Rotationsachse nach Prothesenimplantation}

In dieser Studie wurde besonderes Augenmerk auf das Rotationsintervall zwischen $-1^{\circ}$ und $+1^{\circ}$ gelegt, da sich Bewegungen in vivo vor allem in diesem Winkelbereich abspielen (Wachowski et al. 2009b). Wie in den oben aufgeführten Ergebnissen (3.6.2.1) bereits erwähnt, war unter dem Einfluss der Bandscheibenprothesen ein auffälliger Versatz der IHA (Rastpolkurve) nach dorsal zu beobachten. Diese Verschiebung wurde bei der Betrachtung kleiner Rotationswinkel besonders deutlich. Abbildung 3-32 zeigt beispielhaft die entsprechenden Rastpolkurven für die Rechtsrotation des

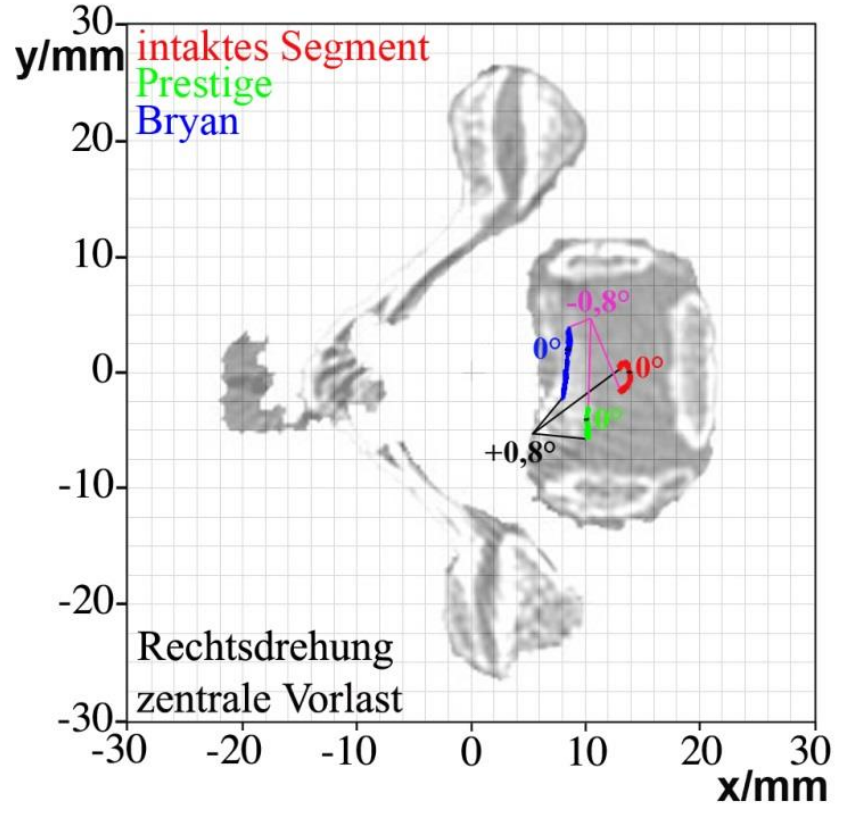

Abb. 3-32: Teilrastpolkurven, axiale Rotation; Präparat 10/07; Vorlast: $20 \mathrm{~N}$ im WZ; Winkelintervall $\pm 0,8^{\circ}$. Nach Implantation der Bandscheibenprothesen ist eine deutliche Verlagerung der IHA in dorsaler Richtung erkennbar. Präparates 10/07 im Winkelintervall $\pm 0,8^{\circ}$.

Neben der sagittalen Positionsänderung war nach Implantation der Bryan ${ }^{\circledR}$ Cervical Disc eine zusätzliche Verlagerung zur rechten Seite erkennbar. Um die kinematische Signifikanz dieser Verschiebung der Rotationsachse genauer beurteilen zu können, musste die dreidimensionale Ausrichtung der IHA nach Einsatz der Bandscheibenprothesen berücksichtigt werden. Die Projektion der momentanen Schraubachse in die seitliche Röntgenaufnahme des Präparates 16/07 ist in 
Abbildung 3-33 dargestellt. Analog zu den Ergebnissen des intakten Bewegungssegments war eine dorso-ventrale Neigung der Rotationsachse zu beobachten (IHA nicht parallel zu $T_{z}(t)$ ). Ebenfalls verlief die Achse bei $\alpha=0^{\circ}$ nahezu parallel zur z-x-Ebene (Abweichung $<5^{\circ}$ ). Im Rahmen des Bewegungszyklus kippte sie maximal um $13^{\circ}$ in lateraler Richtung. Darüber hinaus zeigte sich ein sagittaler Versatz ( $x$-Komponente) in dorsaler Richtung von 5,9 mm unter Prestige ${ }^{\circledR}$ LP und 8,15 mm nach Einsatz der Bryan ${ }^{\circledR}$ Cervical Disc, im Vergleich zum intakten Segment.
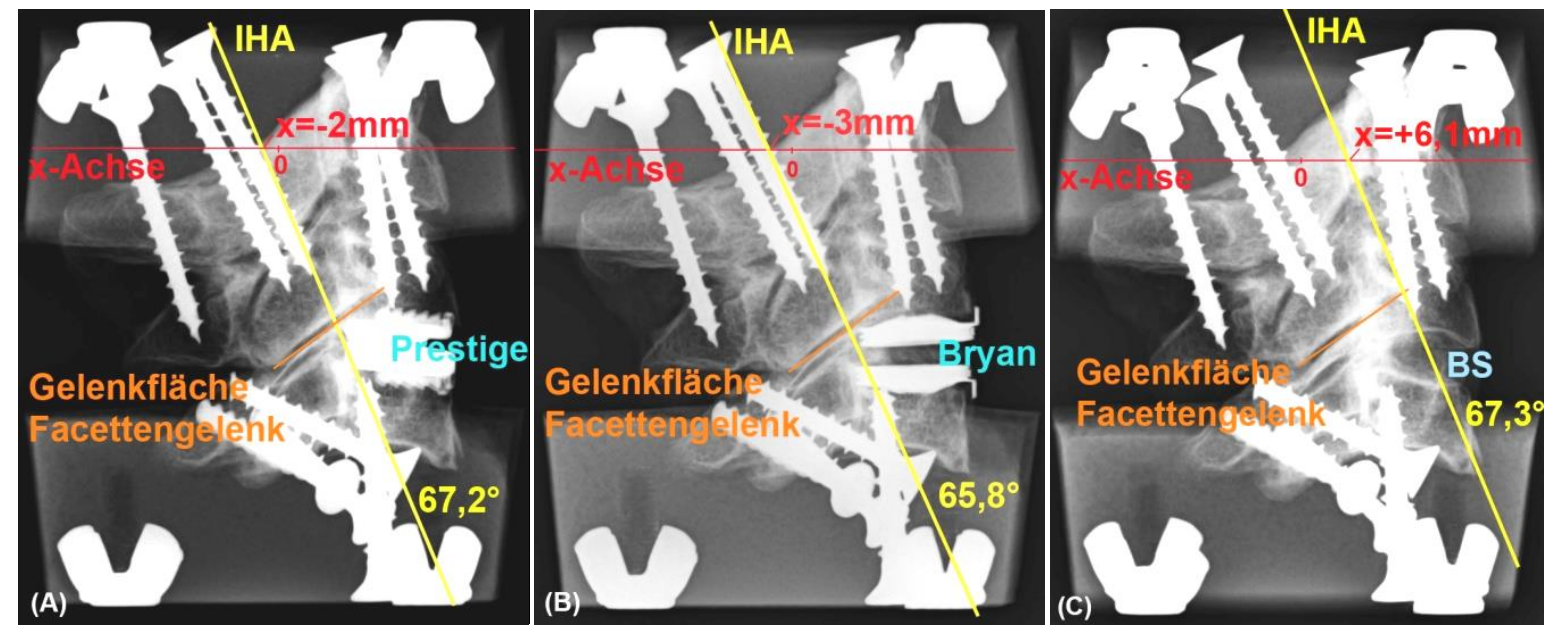

Abb. 3-33: Röntgenaufnahme seitlich; Präparat 16/07; initiale Ausrichtung der IHA nach Implantation der Prestige ${ }^{\circledR}$ LP (A) und Bryan ${ }^{\circledR}$ Cervical Disc (B) im Vergleich zum intakten Segment (C) bei axialer Rotation. Während die Ausrichtung der initialen Rotationsachse vor und nach Prothesenimplantation annährend gleich blieb, zeigte sich nach Implantation der Prothesen eine deutliche Verlagerung der IHA nach dorsal. (Abbildung modifiziert nach Wachowski et al. 2013, Abb. 4)

Die Neigung der Schraubachse in der Sagittalebene änderte sich nach der Implantation der Bandscheibenprothesen nicht signifikant $\left(\approx-69^{\circ}\right)$ und war weiterhin unabhängig von Betrag und Position der axialen Vorlast (Tab. 3-7). Die Konstanz der IHA-Ausrichtung aller acht Versuchsobjekte ließ konstatieren, dass der Einsatz der verwendeten Bandscheibenprothesen im Rotationsintervall $\pm 0,8^{\circ}$ in erster Linie zu einer Translation, also Parallelverschiebung, der momentanen Schraubachsen, statt zu einer Änderung der Ausrichtung führte.

\begin{tabular}{|c|c|c|c|c|}
\hline & \multicolumn{3}{|c|}{ Vorlast $\mathrm{F}_{\mathrm{z}}=20 \mathrm{~N}$} & $F_{2}=60 \mathrm{~N}$ \\
\hline & neutral & ventral & dorsal & neutral \\
\hline Vorlastposition & 0 & $20 \mathrm{~mm}$ & $-20 \mathrm{~mm}$ & 0 \\
\hline $\begin{array}{l}\text { IHA-Ausrichtung } \\
\text { Intaktes Segment }\end{array}$ & $\begin{array}{c}-67,4^{\circ} \\
\sigma=10,7^{\circ}\end{array}$ & $\begin{array}{c}-68,1^{\circ} \\
\sigma=9,55^{\circ}\end{array}$ & $\begin{array}{c}-66,9^{\circ} \\
\sigma=11,27^{\circ}\end{array}$ & $\begin{array}{c}-66,9^{\circ} \\
\sigma=11,3^{\circ}\end{array}$ \\
\hline $\begin{array}{l}\text { IHA-Ausrichtung } \\
\text { Prestige } ₫ \mid \mathrm{P}\end{array}$ & $\begin{array}{c}-67,2^{\circ} \\
\sigma=7,98^{\circ}\end{array}$ & $\begin{array}{c}-70,0^{\circ} \\
\sigma=6,52^{\circ}\end{array}$ & $\begin{array}{c}-72,0^{\circ} \\
\sigma=8,52^{\circ}\end{array}$ & $\begin{array}{l}-69,4^{\circ} \\
\sigma=8,79^{\circ}\end{array}$ \\
\hline $\begin{array}{l}\text { IHA-Ausrichtung } \\
\text { Bryan }{ }^{\circledR} \text { Cervical Disc }\end{array}$ & $\begin{array}{c}-69,3^{\circ} \\
\sigma=7,90^{\circ}\end{array}$ & $\begin{array}{c}-68,0^{\circ} \\
\sigma=6,37^{\circ}\end{array}$ & $\begin{array}{c}-69,8^{\circ} \\
\sigma=10,9^{\circ}\end{array}$ & $\begin{array}{c}-70,9^{\circ} \\
\sigma=9,60^{\circ}\end{array}$ \\
\hline
\end{tabular}

Tab. 3-7: Neigungswinkel der IHA zur Horizontalebene, axiale Rotation; Die initiale Ausrichtung der IHA wurde weder durch den Vorlastbetrag, die Vorlastposition, noch durch die Implantation der beiden Bandscheibenprothesen nennenswert verändert. Standardabweichung $(\sigma)$ (Abb. modifiziert nach Wachowski et al. 2013, Tabelle 1)

Die Berechnung der Translationsvektoren $\Delta \vec{r}_{p i n}\left(0^{\circ}\right)=\left(\Delta \mathrm{y}_{\mathrm{pin}}, \Delta \mathrm{x}_{\mathrm{pin}}\right)=\left(\mathrm{y}_{\mathrm{pn}}\left(0^{\circ}\right)-\mathrm{y}_{\text {in }}\left(0^{\circ}\right), \mathrm{x}_{\mathrm{pn}}\left(0^{\circ}\right)-\mathrm{x}_{\mathrm{in}}\left(0^{\circ}\right)\right)$ und $\Delta \vec{r}_{\text {bin }}\left(0^{\circ}\right)=\left(\Delta \mathrm{y}_{\text {bin }}, \quad \Delta \mathrm{x}_{\mathrm{bin}}\right)=\left(\mathrm{y}_{\mathrm{bn}}\left(0^{\circ}\right)-\mathrm{y}_{\mathrm{in}}\left(0^{\circ}\right), \mathrm{x}_{\mathrm{bn}}\left(0^{\circ}\right)-\mathrm{x}_{\mathrm{in}}\left(0^{\circ}\right)\right)$ bestätigte eine signifikante Verschiebung der Rotationsachsen nach dorsal ( $p<1 \%)$. Abbildung 3-34 zeigt die initiale IHA-Position 
der acht Präparate nach Prothesenimplantation bei $\alpha=0^{\circ}$. Des Weiteren konnte ein signifikanter Unterschied $(p<5 \%)$ zwischen der Verlagerung durch Prestige ${ }^{\circledast}$ LP und Bryan ${ }^{\circledR}$ Cervical Disc $\left(\Delta x_{p i}-\Delta x_{b i}\right.$ $=-5.9 \mathrm{~mm}+8.15 \mathrm{~mm}=2.25 \mathrm{~mm}$ ) ermittelt werden. Der Versatz der IHA in lateraler Richtung wies hingegen keine Signifikanz auf.

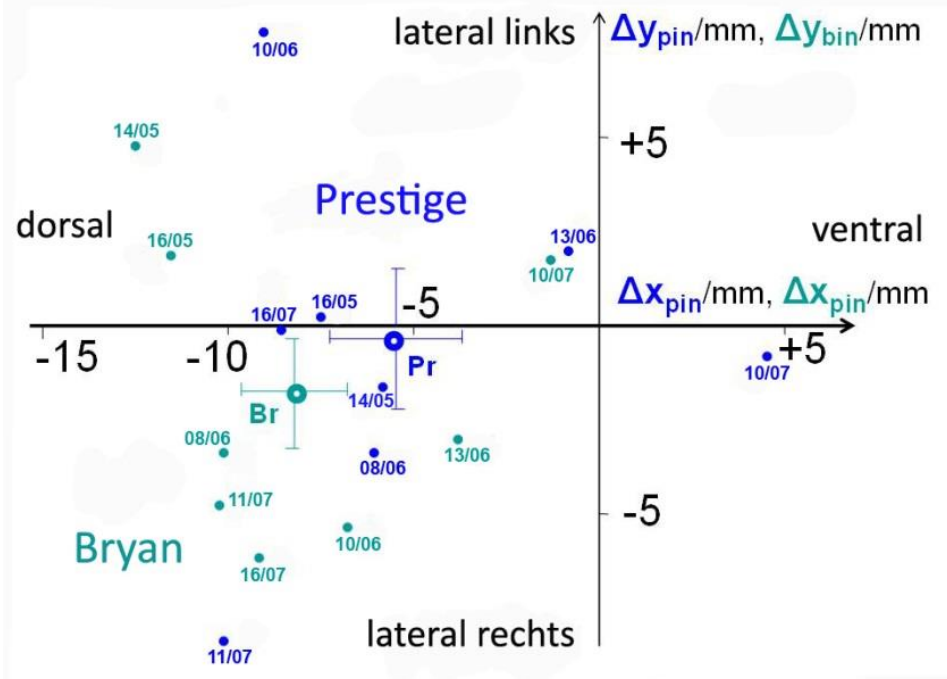

Abb. 3-34: Versatz der initialen Rotationsachse, axiale Rotation; Darstellung der IHA-Verlagerung nach TDA in Abhängigkeit der Koordinaten des jeweils intakten Präparates (Koordinatenursprung). Es ist eine deutliche Dorsalverschiebung nach TDA zu erkennen. Unter Bryan ${ }^{\circledR}$ Cervical Disc kommt es zusätzlich zu einer Verlagerung nach rechts. (Abbildung modifiziert nach Wachowski et al. 2013, Abb. 7)

Darüber hinaus wurde die Verschiebung des zentralen Abschnittes der Rastpolkurve (Rotationsintervall $\pm 0,8^{\circ}$ ) nach Implantation der Bandscheibenprothesen maßgeblich durch die Position der axialen Vorlast beeinflusst. Dabei zeigte sich eine ähnliche Tendenz, wie bei den intakten Präparaten. Mit zunehmender Extensionsstellung des oberen Wirbelkörpers (dorsale Vorlastposition) verlagerte sich die Rastpolkurve weiter nach hinten. Gleichermaßen führte eine Prä-Flexion zu einer Abnahme des dorsalen Versatzes. Zur genaueren Quantifizierung erfolgte die Berechnung der IHAVerlagerung in Abhängigkeit der Vorlastposition. Die y-Komponente des entsprechenden Verschiebungs-Vektors blieb jeweils vernachlässigbar klein und demzufolge ohne Signifikanz. Daher konzentrieren sich die Ausführungen auf den Versatz der Rastpolkurve bei $\alpha=0^{\circ}$ in sagittaler Richtung (x-Komponente). Der Grad der Flexions- bzw. Extensionsstellung vor Rotationsbeginn zeigte im intakten Segment keinen nennenswerten Einfluss auf die Verlagerung der zentralen RPK. Der Versatz von 1,56 mm zwischen maximal flektierter und extendierter Ausgangslage war zu gering um das Signifikanzlevel zu erreichen. Mit Implantation der Prothesen stieg die Verschiebung der zentralen Rastpolkurve auf Werte oberhalb des Signifikanzniveaus, die teilweise um das Vierfache höher waren als bei den intakten Präparaten (Tab. 3-8). Dabei zeigte sich in allen Messungen ein geringeres Ausmaß der RPK-Verschiebung bei Prestige ${ }^{\circledR}$ LP, vergleichen mit Bryan ${ }^{\circledR}$ Cervical Disc. Im Gegensatz zum physiologischen Präparat war zudem die Differenz der Drehachsenposition zwischen ventraler und dorsaler Belastung bei Bryan ${ }^{\circledR}$ Cervical Disc signifikant höher $\left(\Delta \mathrm{x}_{\mathrm{fe}}(\right.$ bryan $)-\Delta \mathrm{x}_{\mathrm{fe}}($ intakt $)=$ $(6,33-1,56) \mathrm{mm}=4.77 \mathrm{~mm}$; t-test, $\mathrm{p}<0,001, \mathrm{n}=14)$. Auch im Vergleich der Extensions-FlexionsDifferenz des intakten Segmentes zu Prestige LP $^{\circledR}$ konnte ein signifikanter Anstieg der IHAVerlagerung beobachtet werden $\left(\Delta \mathrm{x}_{\mathrm{fe}}(\right.$ prestige $)-\Delta \mathrm{x}_{\mathrm{fe}}($ intakt $\left.)=4,07 \mathrm{~mm}, \mathrm{p}=0,02\right)$. 


\begin{tabular}{|l|c|c|c|}
\hline \multirow{2}{*}{ Segment } & \multicolumn{3}{|c|}{ Versatz der zentralen Rastpolkurve } \\
\cline { 2 - 4 } & $\boldsymbol{\Delta} \mathbf{x}_{\mathrm{ne}} / \mathbf{m m}$ & $\boldsymbol{\Delta} \mathbf{x}_{\mathrm{fn}} / \mathbf{m m}$ & $\boldsymbol{\Delta} \mathbf{x}_{\mathrm{fe}} / \mathbf{m m}$ \\
\hline Intakt & $\mathbf{1 , 0 8}$ & $\mathbf{0 , 5 0 3}$ & $\mathbf{1 , 5 6}$ \\
& $\sigma=1,682$ & $\sigma=1,542$ & $\sigma=2,345$ \\
& $\mathrm{t}=1,82 ;$ n.s. & $\mathrm{t}=0,92 ; \mathrm{n} . \mathrm{s}$. & $\mathrm{t}=1,88 ; \mathrm{n} . \mathrm{s}$. \\
\hline Prestige $^{\circledR} \mathrm{LP}$ & 2,31 & 3,32 & 5,63 \\
& $\sigma=2,660$ & $\sigma=2,747$ & $\sigma=3,713$ \\
& $\mathrm{t}=2,456 ; \mathrm{p}<0,05$ & $\mathrm{t}=3,418 ; \mathrm{p}<0,02$ & $\mathrm{t}=4,289 ; \mathrm{p}<0,01$ \\
\hline Bryan $^{\circledR}$ Cervical Disc & $\mathbf{2 , 6 3}$ & $\mathbf{3}, 71$ & $\mathbf{6 , 3 3}$ \\
& $\sigma=2,223$ & $\sigma=2,064$ & $\sigma=2,100$ \\
& $\mathrm{t}=3,349 ; \mathrm{p}<0,015$ & $\mathrm{t}=5,079 ; \mathrm{p}<0,005$ & $\mathrm{t}=8,53 ; \mathrm{p}<0,001$ \\
\hline
\end{tabular}

Tab. 3-8: Differenz der Rotationsachsenposition zwischen zentraler und dorsaler $\left(\Delta \mathrm{x}_{\mathrm{ne}}\right)$, ventraler und zentraler $\left(\Delta \mathrm{x}_{\mathrm{ff}}\right)$, sowie ventraler und dorsaler Belastung $\left(\Delta \mathrm{x}_{\mathrm{fe}}\right), \sigma=$ Standardabweichung, $\mathrm{t}=\mathrm{t}$-Wert, $\mathrm{p}=\mathrm{p}$-Wert (Signifikanzlevel)

Am intakten Segment führte die Verlagerung der Vorlast lediglich zu einer nicht signifikanten Verschiebung der Rotationsachse bei $0^{\circ}$. Nach Implantation der Prothesen zeigte sich eine stärkere Abhängigkeit der Lage der momentanen Rotationsachse von der Position der Vorlast. Die Implantation der Bryan® führte zu einem noch höheren, signifikanten Unterschied als die der Prestige ${ }^{\circledR}$ LP. (Abbildung modifiziert nach Wachowski et al. 2013, Tabelle 2)

\subsubsection{Schwerpunkt}

Der Schwerpunkt $r(s)$ einer Kurve wird geometrisch betrachtet als Massenmittelpunkt definiert. Er spiegelt den Punkt wider, an welchem eine Figur ausbalanciert ist und nicht mehr verkippt. Mathematisch betrachtet entspricht er der Mittelung aller Punkte einer Figur/Kurve. Die Berechnung der Schwerpunktkoordinaten $(x(s), y(s))$ der zweidimensionalen Rastpolkurve wurde anhand der Formeln: $\mathrm{Xs}=\int(\mathrm{xds}) / \mathrm{L}$ und $\mathrm{ys}=\int(\mathrm{yds}) / \mathrm{L}$ vorgenommen (Weidl 2003), wobei L die Gesamtlänge der Rastpolkurve darstellte. In allen Messungen war kein signifikanter Unterschied der Schwerpunktkoordinaten $(x(s), y(s))$ für die Links- und Rechtsrotation nachzuweisen $\left(p_{x}(s)=0,825\right.$, $\left.p_{y}(s)=0,770\right)$, sodass postuliert werden konnte, anhand der Ergebnisse einer Rotationsrichtung auf die Position des Schwerpunktes der gesamten RPK (Links- und Rechtsrotation) zurückschließen zu können.

In Tabelle 3-9 sind die Schwerpunktkoordinaten der Rastpolkurve für die Rechtsrotation des intakten Segmentes und nach der Implantation der beiden Bandscheibenprothesen unter einer zentralen Belastung von $20 \mathrm{~N}$ aufgeführt.

\begin{tabular}{|l|c|c|}
\hline \multirow{2}{*}{ Segment } & \multicolumn{2}{c|}{ Koordinaten des Schwerpunktes (20 N im WZ) } \\
\cline { 2 - 3 } & $\mathbf{x ( s )}$ & $\mathbf{y}(\mathbf{s})$ \\
\hline Intakt & $\mathbf{0 , 8 3}(\sigma=3,38)$ & $-0,37(\sigma=9,29)$ \\
\hline Prestige $^{\oplus}$ LP & $-2,94(\sigma=5,14)$ & $-0,55(\sigma=6,75)$ \\
\hline Bryan $^{\oplus}$ Cervical Disc & $-\mathbf{5 , 1 3}(\sigma=4,84)$ & $-\mathbf{2 , 2 9}(\sigma=6,94)$ \\
\hline
\end{tabular}

Tab. 3-9: Koordinaten des Schwerpunktes für Rechtsrotation bei einer axialen Belastung von $20 \mathrm{~N}$ im WZ; Vergleich zwischen intaktem Segment, Prestige ${ }^{\circledR}$ LP und Bryan ${ }^{\circledR}$ Cervical Disc, jeweilige Standardabweichung $(\sigma)$ in Klammern. Nach Implantation der Prothesen zeigte sich ein deutlicher Versatz des Schwerpunktes in xRichtung (nach dorsal). Unter Bryan ${ }^{\circledR}$ war zudem eine auffällige Verlagerung in y-Richtung zu erkennen (nach rechts). 
Zur Abschätzung der Schwerpunktverlagerung, wurde nachfolgend die Abhängigkeit der Koordinaten von lediglich einem einzigen Parameter (Vorlastposition oder Art des Präparatezustandes: intaktes Segment/TDA) betrachtet. Dabei zeigte sich weder infolge der Prothesenimplantation, noch durch den Versatz der axialen Vorlast eine signifikante Änderung der $y$-Koordinate $(p \geq 0,307)$. Dem gegenüber wies die $x$-Koordinate des Schwerpunktes eine deutliche Abhängigkeit von der Art des Bandscheibenersatzes auf $\left(x_{s}\right.$ (intakt-prestige $)=-4,39 \mathrm{~mm}, \mathrm{p}=3,2 \mathrm{e}^{\wedge}(-06) ; \mathrm{x}_{\mathrm{s}}$ (intakt-bryan) $=-6,51 \mathrm{~mm}$, $\left.p=2,7 e^{\wedge}(-11)\right)$. Die Implantation der Bandscheibenprothesen führte damit sowohl im Vergleich zum intakten Segment, als auch im Vergleich der beiden Modelle unter einander zu einer signifikanten Verschiebung des Schwerpunktes in sagittaler Richtung. Die Verlagerung der axialen Vorlast aus dem Widerstandszentrum heraus blieb analog zur y-Koordinate ohne signifikanten Einfluss auf die $x$ Koordinate $\left(p_{x} s(z-v V L)=0,51 ; p_{x} s(z-d V L)=0,44 ; p_{x} s(v-d V L)=0,18\right)$

\subsubsection{Initiale Migrationsrate (IMR) der Rotationsachse}

Zur weiteren Analyse der kinematischen Eigenschaften der Rotationsachse wurde aus der Migrationsgeschwindigkeit, als feststehender Betrag des IHA-Versatzes entlang der Rastpolkurve ( $\left.\vec{v}_{m}=d \vec{s} / d t\right)$ und der Winkelgeschwindigkeit $(\omega=d \alpha / d t)$ die Migrationsrate berechnet: $\vec{v}(\alpha)=\vec{v}_{m} / \omega=d \vec{s} / d \alpha$. Für die initiale Migrationsrate ergab sich folglich: $\overline{\vec{v}(0)}=\overline{\Delta \vec{s} / \Delta \alpha}$. Für jedes Versuchspräparat erfolgte die entsprechende Vermessung des Versatzes der IHA-Position entlang der jeweiligen Rastpolkurve $\left(\Delta \vec{s}=\left(\Delta s_{x}, \Delta s_{y}\right)\right)$ für den Winkelabstand $\Delta \alpha \mathrm{L}=(0,8$ deg - 0$)$ bei der Drehung nach links und $\Delta \alpha R=(-0,8$ deg -0$)$ bei der Rechtsdrehung. In diesem Rotationsbereich war der Ausgleichsvorgang nach Bewegungsumkehr abgeklungen und hatte folglich keinen Einfluss mehr. Die IMR der Richtungskomponenten berechneten sich aus $v_{y}=\Delta s_{y} / \Delta \alpha$ und $v_{x}=\Delta s_{x} / \Delta \alpha$ (Wachowski et al. 2013).

Für die x-Komponente ergaben sich in allen Präparaten lediglich kleine Werte, die ohne Signifikanz blieben. Die Berechnung der initialen Migrationsrate in y-Richtung wies demgegenüber sowohl vor, als auch nach der Prothesenimplantation höhere Ergebnisse auf und zeigte eine deutliche Abhängigkeit von der Vorlastposition (siehe Abb. 3-35). Während unter ventraler Belastung lediglich beim intakten Segment eine signifikante Migration $(p<0,05)$ von durchschnittlich $2 \mathrm{~mm} / \mathrm{deg} z u$ beobachten war, zeigte sich bei zentraler und dorsaler Vorlastposition, sowohl beim intakten Segment, als auch nach der Prothesenimplantation eine signifikante Migration ( $p<0,01$ bzw. $p<0,001)$. Das Ausmaß der stattgefundenen Migration war in dem beobachteten Rotationsbereich $\left(0,8^{\circ}\right.$ bis $\left.-0,8^{\circ}\right)$ unter dorsaler Vorlast am größten (Abb. 3-35). Gleichermaßen war eine signifikante Veränderung durch die alleinige Implantation der Bandscheibenprothesen zu beobachten. Speziell bei dem Vergleich der initialen Migrationsrate des intakten Segmentes mit Bryan ${ }^{\circledast}$ Cervical Disc 
System wurde ein signifikanter Unterschied erkennbar $\left(\left\langle\Delta \mathrm{v}_{\mathrm{ybi}}\right\rangle=2,33 \mathrm{~mm} / \mathrm{deg}, \mathrm{SD}=5,38 \mathrm{~mm} / \mathrm{deg}\right.$, student t-test: $d f=47, p<0,01)$. Die Gegenüberstellung beider Prothesen $\left(\Delta v_{y b p}=v_{y, b r y a n}-v_{y, \text { prestige }}\right)$ zeigte ebenfalls eine signifikante Differenz $\left(\left\langle\Delta \mathrm{v}_{\mathrm{ybp}}\right\rangle=1,47 \mathrm{~mm} / \mathrm{deg}, \mathrm{SD}=5,19 \mathrm{~mm} / \mathrm{deg}\right.$, student t-test: $d f=47, p=0,057))$. Die Abweichung der IMR zwischen intaktem Segment und Prestige LP ${ }^{\circledR}$ blieb hingegen ohne Signifikanz $\left(\left\langle\Delta \mathrm{v}_{y p i}\right\rangle=0.89 \mathrm{~mm} / \mathrm{deg}, \mathrm{SD}=5,23 \mathrm{~mm} / \mathrm{deg}\right.$, student test: $\mathrm{df}=47$, n.s. $)$ (Wachwoski et al. 2013).

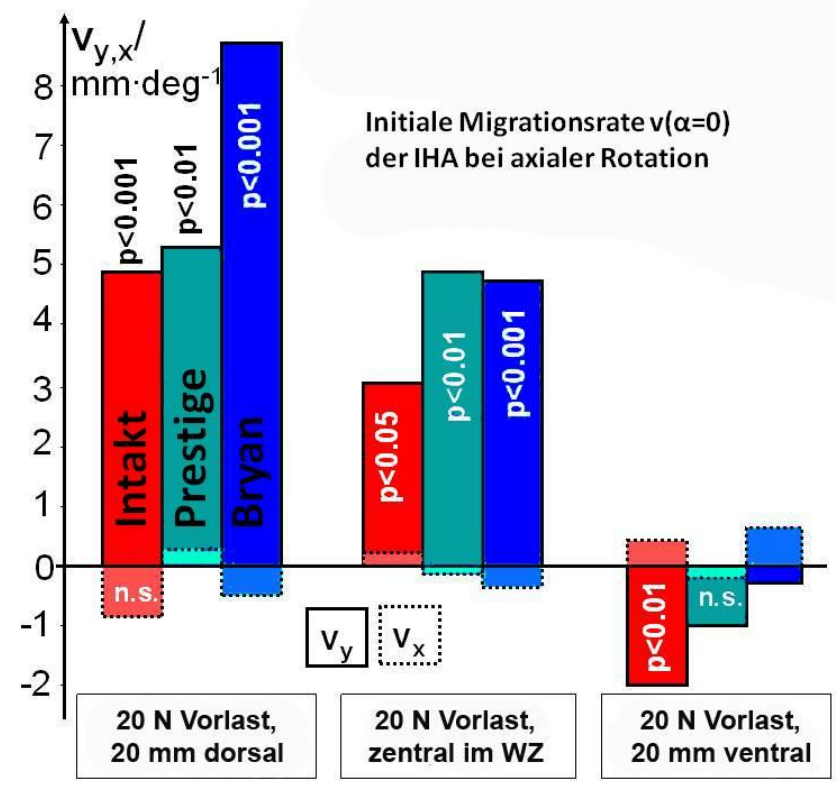

Abb. 3-35: Initiale Migrationsrate bei axialer Rotation in Abhängigkeit der Vorlastposition. Komponenten $\mathrm{v}_{\mathrm{y}}(0)$ (geschlossene Balken) und $\mathrm{v}_{\mathrm{x}}(0)$ (gestrichelte Balken) des intakten Segmentes (rot), sowie nach Implantation der Prestige ${ }^{\circledR}$ LP (grün) und Bryan ${ }^{\circledR}$ Cervical Disc (blau). Positive Werte sprechen für eine gleichsinnige IHAWanderung mit der Rotationsbewegung (z.B. Linksrotation: Migration nach links), negative Werte für eine gegenläufige Migration (z.B. Linksrotation: Migration nach rechts). Die unterschiedlichen Signifikanzstufen (p-Werte) geben an, mit welcher Signifikanz überhaupt eine Migration unter der jeweiligen Vorlast stattgefunden hat (gegen Null getestet). Die Höhe der Balken geben das Ausmaß (mm/deg) der jeweils stattgefundenen Migration wieder. (Abbildung modifiziert nach Wachowski et al. 2013, Abb. 8)

\subsubsection{Drehwinkel-Drehmoment-Kennlinie, Bewegungsausmaß und Steifigkeit}

Die Implantation der Bandscheibenprothesen hatte einen deutlichen Einfluss auf das Bewegungsausmaß und die minimale Steifigkeit. Durch die Resektion der Bandscheibe und deren Ersatz durch eine Prothese stieg das Bewegungsausmaß bei einem gleichbleibenden Drehmomentintervall und zentraler Belastung $(20 \quad N)$ durchschnittlich von $4,28^{\circ}$ auf $5,62^{\circ}$ (Prestige LP $^{\circledR}$ ) bzw. 6,08 ${ }^{\circ}\left(\right.$ Bryan $\left.^{\circledR}\right)$. Dies entsprach einer Zunahme von annähernd 32\% bzw. 42\%. Der sigmoide Verlauf der Alpha(T)-Kennlinie blieb weiterhin erhalten. Simultan kam es zu einer

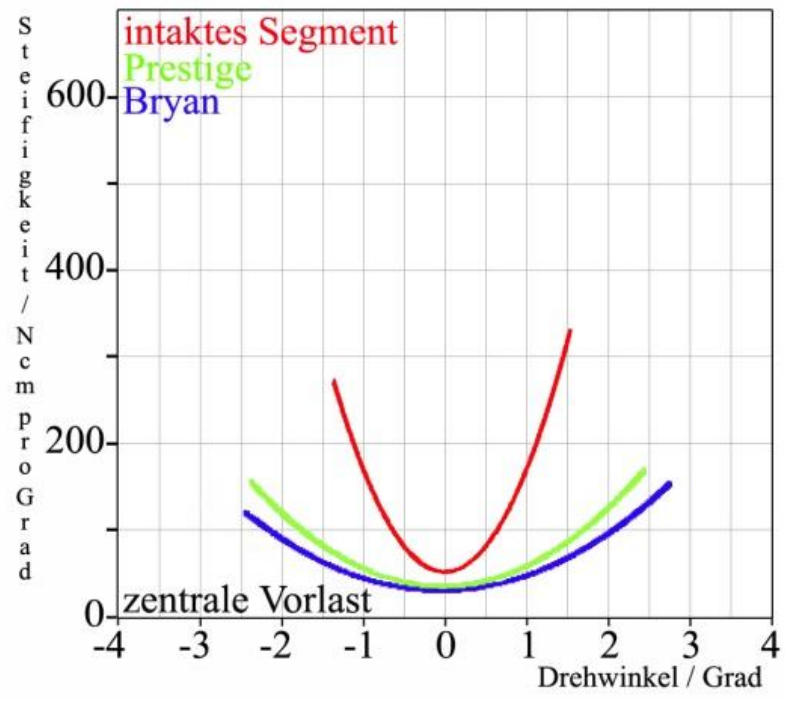

Abb. 3-36: Steifigkeitsdiagramm, axiale Rotation; Vergleich: intaktes Segment - Prestige $®$ LP - Bryan®; Präparat 10/07; Vorlast: $20 \mathrm{~N}$ im WZ. Unter Prestige ${ }^{\circledR}$ LP und Bryan® kam es zu einem deutlichen Abfall der Steifigkeit. 
deutlichen Reduktion der minimalen Steifigkeit. Der Bandscheibenersatz durch Prestige LP® führte zu einer Abnahme der minimalen Steifigkeit um etwa 36\%. Die anschließende Implantation der Bryan ${ }^{\circledR}$ hatte einen Abfall um weitere 9\% zur Folge. Eine Erhöhung der zentralen Vorlast ging mit einem ähnlichen Effekt auf ROM und Steifigkeit einher, wie vor der Implantation. Dies galt auch für die sagittale Verschiebung in ventraler Richtung. Die Daten in Tabelle 3-10 und Tabelle 3-11 lassen erkennen, dass unter ventraler Vorlast die prozentuale Veränderung der initialen Steifigkeit und des Bewegungsausmaßes nahezu den Werten der intakten Segmente entsprechen. Bei dorsaler und lateraler Vorlastposition war hingegen eine deutlichere Reduktion des ROM zu beobachten, als beim intakten Segment. Dies korrelierte mit einem stärkeren Anstieg der minimalen Steifigkeit in Extensionsstellung. Bei einer lateralen Belastung fand sich trotz geringerer ROM keine signifikante Änderung der minimalen Steifigkeit. Hier nahm die minimale Steifigkeit nur gering zu. Der Vergleich der relativen Steifigkeit nach Prothesenimplantation mit dem intakten Segment zeigte bei der Prestige ${ }^{\circledR}$ LP eine annähernd gleichbleibende und von der Vorlastposition unabhängige Abnahme der Segmentstabilität (siehe Tab. 3-12). Hingegen war nach der

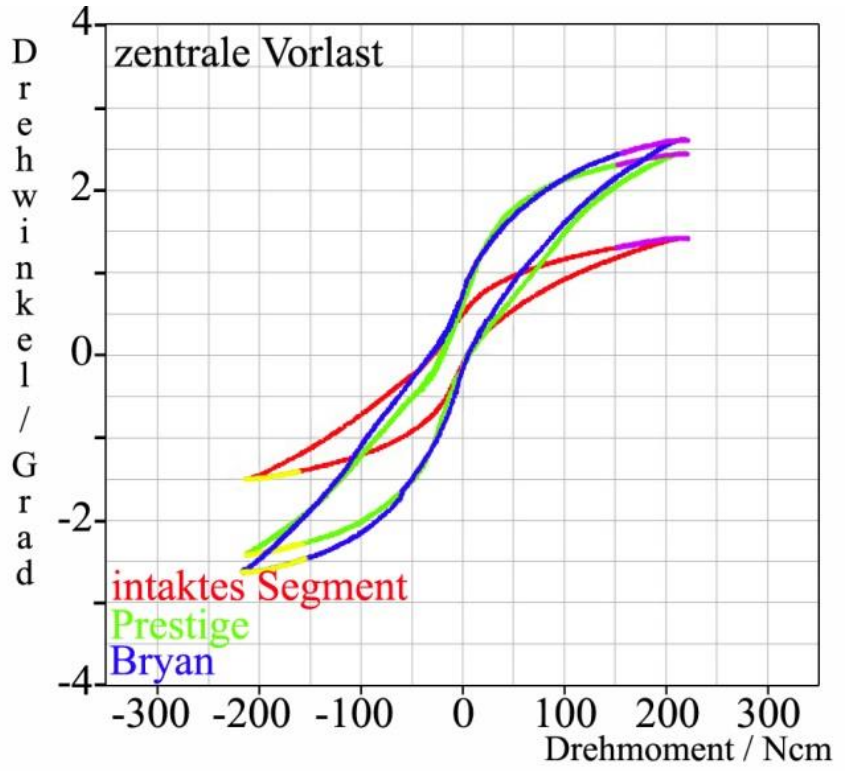

Abb. 3-37: Alpha(T)-Diagramm, axiale Rotation; Vergleich: intaktes Segment - Prestige ${ }^{\circledR}$ LP - Bryan ${ }^{\circledR}$; Präparat 10/07; axialen Vorlast: $20 \mathrm{~N}$ im WZ. Analog zum Abfall der Steifigkeit nach Implantation der Bandscheibenprothesen kam es zu einem Anstieg des Bewegungsausmaßes. Implantation der Bryan ${ }^{\circledR}$ Cervical Disc ein deutlich höherer Effekt auf die Segmentsteifigkeit infolge einer Vorlastverlagerung zu beobachten. 


\begin{tabular}{|c|c|c|c|c|}
\hline \multirow{3}{*}{ Intaktes Segment } & & \multirow{2}{*}{$\begin{array}{l}20 \mathrm{~N} \text { im WZ } \\
4,28(\sigma=1,54)\end{array}$} & \multirow{2}{*}{$\begin{array}{l}20 \mathrm{~N} 20 \mathrm{~mm} \text { ventral } \\
4,74(\sigma=1,5)\end{array}$} & $20 \mathrm{~N} 20 \mathrm{~mm}$ dorsal \\
\hline & ROM [deg] & & & $3,56(\sigma=1,53)$ \\
\hline & ROM rel [\%] & 100 & $112,86(\sigma=9,04)$ & $83,18(\sigma=11)$ \\
\hline \multirow[t]{2}{*}{ Prestige ${ }^{\circledR}$ LP } & ROM [deg] & $5,62(\sigma=2,38)$ & $6,26(\sigma=2,44)$ & $3,83(\sigma=1,24)$ \\
\hline & ROM rel [\%] & 100 & $111,39(\sigma=9,14)$ & $68,15(\sigma=9,93)$ \\
\hline \multirow{2}{*}{$\begin{array}{l}\text { Bryan }{ }^{\circledast} \text { Cervical } \\
\text { Disc }\end{array}$} & ROM [deg] & $6,08(\sigma=2,6)$ & $6,84(\sigma=2,4)$ & $4,14(\sigma=1,61)$ \\
\hline & ROM rel [\%] & 100 & $112,50(\sigma=16,91)$ & $68,09(\sigma=14,48)$ \\
\hline
\end{tabular}

Tab. 3-10: Range of motion (ROM) nach Implantation der Prestige ${ }^{\circledR}$ LP und Bryan® Cervical Disc unter verschiedenen Vorlastpositionen; Vergleich zum intakten Bewegungssegment; Standardabweichung $(\sigma)$ in Klammern; axiale Rotation. Die Implantation der Prothesen ging mit einer Zunahme des Bewegungsausmaßes einher. Die Abhängigkeit von der VL-Position blieb tendenziell erhalten. Der prozentuale Anstieg des ROM unter ventraler VL blieb annähernd konstant, während bei dorsaler VL-Position ein stärkerer Abfall des Bewegungsausmaßes unter Prestige $\AA^{2}$ LP und Bryan $®$ zu beobachten war.

\begin{tabular}{|c|c|c|c|c|}
\hline & & $20 \mathrm{~N}$ im WZ & $20 \mathrm{~N} 20 \mathrm{~mm}$ ventral & 20 N 20 mm dorsal \\
\hline \multirow[t]{2}{*}{ Intaktes Segment } & {$[\mathrm{Ncm} / \mathrm{deg}]$} & $42,75(\sigma=9,48)$ & $30,38(\sigma=7,05)$ & $66(\sigma=26,99)$ \\
\hline & [Ncm/deg] rel [\%] & 100 & $73(\sigma=17)$ & $150(\sigma=42)$ \\
\hline \multirow[t]{2}{*}{ Prestige $^{\circledR}$ LP } & [Ncm/deg] & $27,25(\sigma=10,9)$ & $20,38(\sigma=7,8)$ & $46,85(\sigma=28,42)$ \\
\hline & [Ncm/deg] rel [\%] & 100 & $75(\sigma=14,96)$ & $172(\sigma=84,53)$ \\
\hline \multirow{2}{*}{$\begin{array}{l}\text { Bryan }{ }^{\circledR} \text { Cervical } \\
\text { Disc }\end{array}$} & [Ncm/deg] & $23,5(\sigma=23,5)$ & $14,75(\sigma=8,81)$ & $54,13(\sigma=39,08)$ \\
\hline & [Ncm/deg] rel [\%] & 100 & $63(\sigma=30,78)$ & $230(\sigma=230,67)$ \\
\hline
\end{tabular}

Tab. 3-11: Steifigkeit $[\mathrm{Ncm} / \mathrm{deg}]$ nach Implantation der Prestige ${ }^{\circledR}$ LP und Bryan ${ }^{\circledR}$ Cervical Disc unter verschiedenen Vorlastpositionen; Vergleich zum intakten Bewegungssegment; Standardabweichung $(\sigma)$ in Klammern; axiale Rotation. Eine ventrale Vorlastposition führte sowohl vor, als auch nach der Prothesenimplantation zu einer Abnahme der Segmentsteifigkeit, während eine dorsal platzierte Vorlast mit einer Steifigkeitszunahme einherging.

\begin{tabular}{|c|c|c|c|c|}
\hline & & $20 \mathrm{~N}$ im WZ & $20 \mathrm{~N} 20 \mathrm{~mm}$ ventral & 20 N 20 mm dorsal \\
\hline \multirow[t]{2}{*}{ Intaktes Segment } & {$[\mathrm{Ncm} / \mathrm{deg}]$} & $42,75(\sigma=9,48)$ & $30,38(\sigma=7,05)$ & $66(\sigma=26,99)$ \\
\hline & [Ncm/deg] rel [\%] & 100 & 100 & 100 \\
\hline \multirow[t]{2}{*}{ Prestige $^{\circledR}$ LP } & {$[\mathrm{Ncm} / \mathrm{deg}]$} & $27,25(\sigma=10,9)$ & $20,38(\sigma=7,8)$ & $46,85(\sigma=28,42)$ \\
\hline & [Ncm/deg] rel [\%] & 63,74 & 67,08 & 70,98 \\
\hline \multirow{2}{*}{$\begin{array}{l}\text { Bryan }^{\circledast} \text { Cervical } \\
\text { Disc }\end{array}$} & {$[\mathrm{Ncm} / \mathrm{deg}]$} & $23,5(\sigma=23,5)$ & $14,75(\sigma=8,81)$ & $54,13(\sigma=39,08)$ \\
\hline & [Ncm/deg] rel [\%] & 54,97 & 48,55 & 82,01 \\
\hline
\end{tabular}

Tab. 3-12: Vergleich der relativen Steifigkeit nach Prothesenimplantation mit dem intakten Segment in Abhängigkeit von der Vorlastposition; Standardabweichung $(\sigma)$ in Klammern; axiale Rotation. Nach Implantation der Bandscheibenprothesen war im Vergleich zum intakten Segment in allen Vorlastkonstellationen eine geringere Steifigkeit zu verzeichnen. 


\subsection{Lateralflexion}

Die Datenerhebung für die Lateralflexion fand unter den gleichen Versuchsbedingungen statt, wie sie für die Messungen der axialen Rotation definiert wurden. Betrag und Position der axialen Vorlast wurden gemäß dem Schema in Abb. 2-10 appliziert und ein Drehmoment $T(x)$ von 2,25 Nm angelegt.

\subsubsection{Lage und Wanderung der Rotationsachse}

Die Rastpolkurven aller acht Versuchspräparate zeigten bei Lateralflexion eine ähnliche Gestalt. Im Vergleich zur axialen Rotation war diese durch ein weniger komplexes, aber dennoch ausgeprägtes Migrationsverhalten gekennzeichnet. Dabei war die RPK überwiegend zentral, im Bereich des oberen Wirbelkörpers (C3) lokalisiert (siehe Abb. 3-38). Zu Beginn des Neigungsvorganges nach links lag die IHA etwas linksversetzt, relativ zentral im Bereich der Grundplatte des dritten Halswirbels.

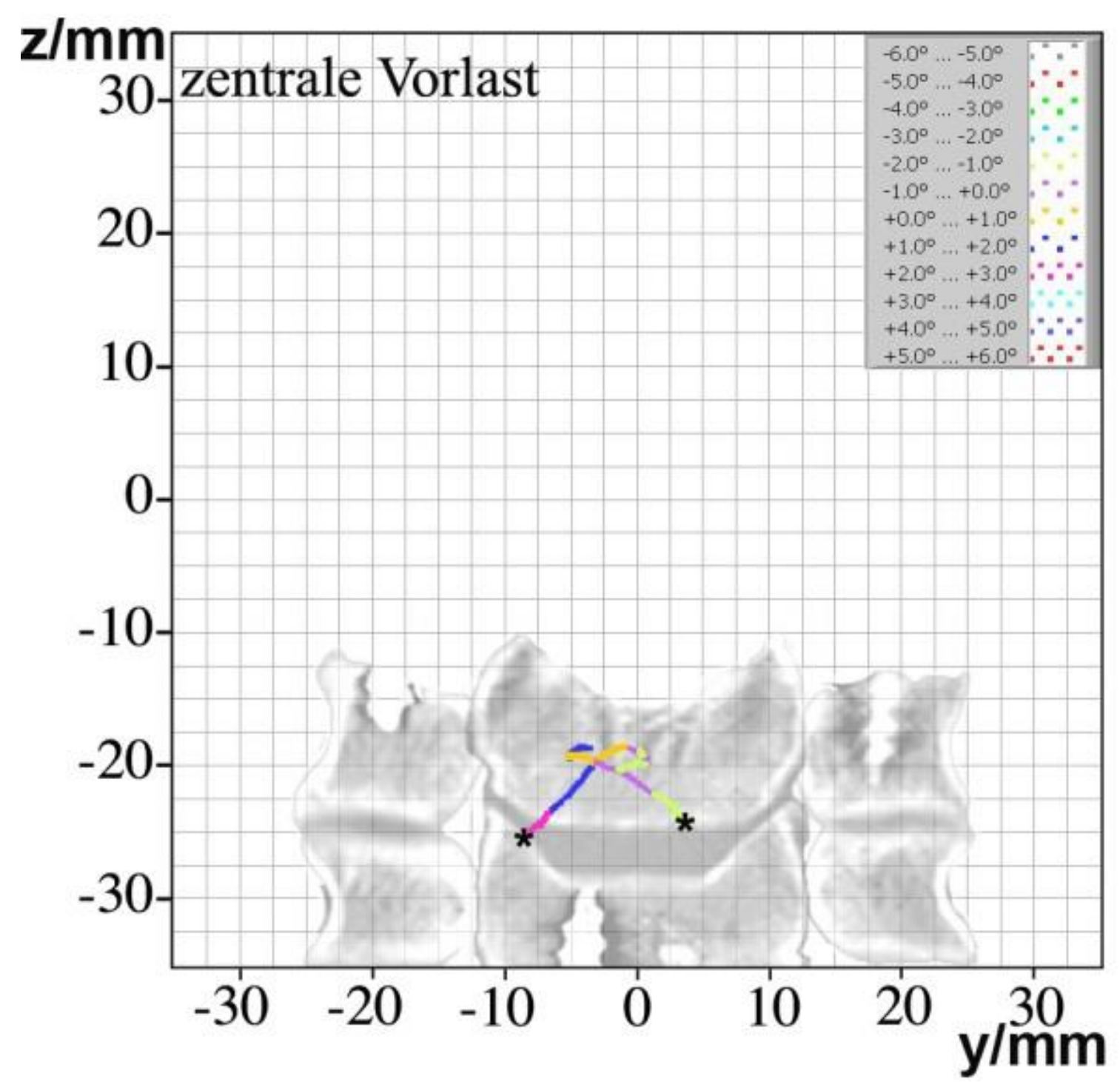

Abb. 3-38: Rastpolkurve, Lateralflexion; intaktes Segment 10/07; Vorlast: 20 N im WZ. Der Startpunkt der Rastpolkurve einer Bewegungsrichtung ist jeweils mit "**" gekennzeichnet. 
Im Verlauf der Bewegung wanderte sie zunächst geradlinig nach rechts-kranial in die Mitte des Wirbelkörpers, um nach einem scharfen Richtungswechsel $\left(\alpha \approx 1^{\circ}\right)$ mittig im rechten Anteil des Corpus vertebrae zu enden. Der anschließende Richtungswechsel führte zu einem Sprung der Rastpolkurve ca. $7 \mathrm{~mm}$ nach kaudal und $5 \mathrm{~mm}$ nach links auf die Höhe des rechten Uncovertebralgelenkes. Von dort bewegte sich die momentane Schraubachse vorerst geradlinig nach links-kranial in die Mitte des dritten Halswirbelkörpers. Dort verlief sie in einem kranial-konvexem Bogen weiter nach links und endete nach einem scharfen Wendepunkt $\left(\alpha \approx-1^{\circ}\right)$ mittig im Wirbelkörper. Die erneute Änderung der Neigungsrichtung resultiert in einem Sprung der Rotationsachse um $5 \mathrm{~mm}$ nach links und 4,5 mm nach kaudal, zurück in die Ausgangsposition.

Der Einfluss der Vorlastposition bzw. deren Betrag auf die Migration der RPK war bei der Lateralflexion weniger stark ausgeprägt als bei Axialrotation. Lediglich ventral positionierte Vorlastbeträge führten zu einer zunehmenden Stauchung der Rastpolkurve, sowohl vertikal, als auch transversal (vgl. Abb. 3-39). Die Implantation der Bandscheibenprothesen hingegen führte zu einem deutlichen Versatz der IHA nach kranial (Presige ${ }^{\circledR}$ LP ca. 5 mm; Bryan ${ }^{\circledR}$ Cervical Disc ca. 8 mm) (Abb. 3-40). Das Aussehen der Rastpolkurve veränderte sich allerdings kaum.

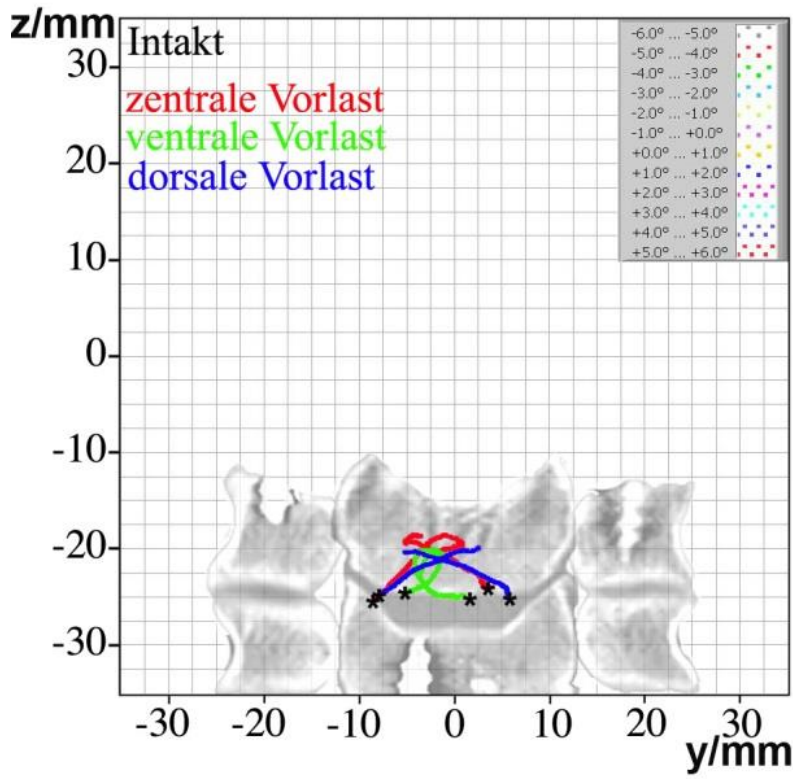

Abb. 3-39: Rastpolkurve, Lateralflexion; intaktes Segment 10/07; axiale Vorlast: 20 N $20 \mathrm{~mm}$ dorsal bzw. ventral und im WZ. Der Startpunkt der Rastpolkurve einer Bewegungsrichtung ist jeweils mit “*“ gekennzeichnet. Der Migrationsverlauf änderte sich bei unterschiedlichen Vorlastpositionen nur geringfügig.

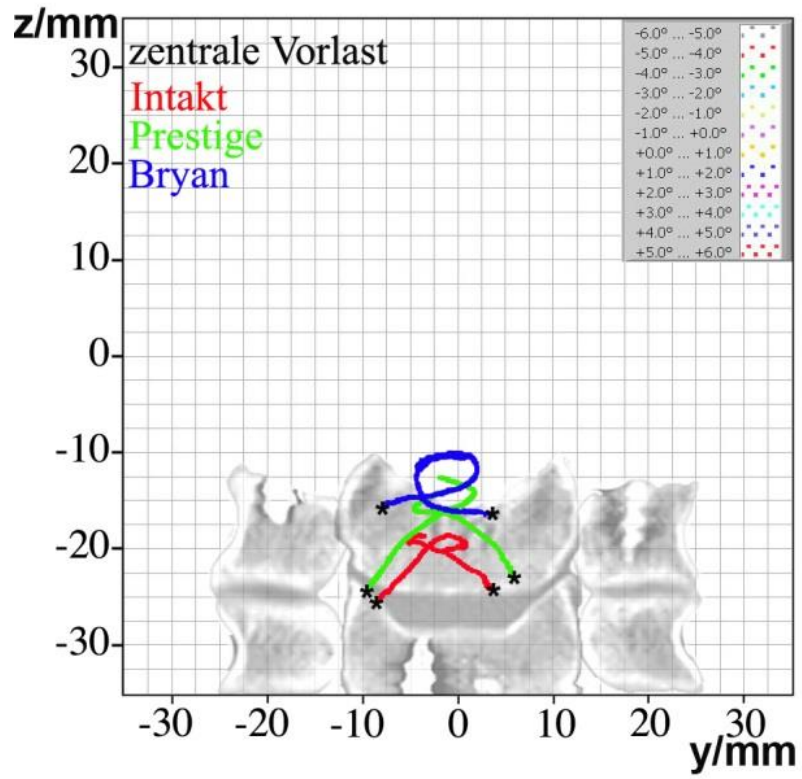

Abb. 3-40: Rastpolkurve, Lateralflexion; Präparat 10/07; axiale Vorlast: 20 N im WZ. Der Startpunkt der Rastpolkurve einer Bewegungsrichtung ist jeweils mit “** gekennzeichnet.

Nach Implantation der Prothesen zeigte sich ein deutlicher Versatz der momentanen Schraubachse nach kranial. Die Gestalt der RPK bleibt hingegen nach Prothesenimplantation annähernd gleich. 


\subsubsection{Ausrichtung der Rotationsachse}

Wie auch bei axialer Rotation, lagen die Rotationsachsen nicht parallel zum anliegenden Drehmoment $\left(T_{x}\right)$, sondern ca. $20-30^{\circ}$ zur Horizontalebene geneigt und verliefen dabei von kraniodorsal nach kaudo-ventral (Abb. 3-41 und Abb. 3-42). Bei der Lateralflexion kam es im Rahmen des Bewegungszyklus $\left(\alpha>1^{\circ}\right.$ bzw. $\left.\alpha<-1^{\circ}\right)$ zusätzlich zu einer deutlich ausgeprägten Ausschwenkung in lateraler Richtung, sodass die Schraubachsen nicht parallel zueinander lagen, sondern eine fächerförmige Regelfläche bildeten. Initial $\left(\alpha=0^{\circ}\right)$ lag die IHA sowohl beim intakten Segment, als auch nach Prothesenimplantation annähernd parallel zur Sagittalebene (Abweichung $\leq 10^{\circ}$ ). Mit zunehmendem Rotationswinkel zeigte sich eine stark verändernde z-Komponente $\left(e_{z}\right)$ des Richtungsvektors, was in einem Schwenken der Rotationsachse nach rechts bzw. links resultierte. Dies entsprach einer Neigung der IHA zur Frontalebene um durchschnittlich $20^{\circ}$ bis $30^{\circ}$ im Sinne einer Auffächerung in der Horizontalebene.

Zusammenfassend kann man sagen, dass weder Verlagerung oder Erhöhung der Vorlast, noch die Implantation der Prothesen zu einer signifikanten Änderung der Ausrichtung der Rotationsachse geführt haben.

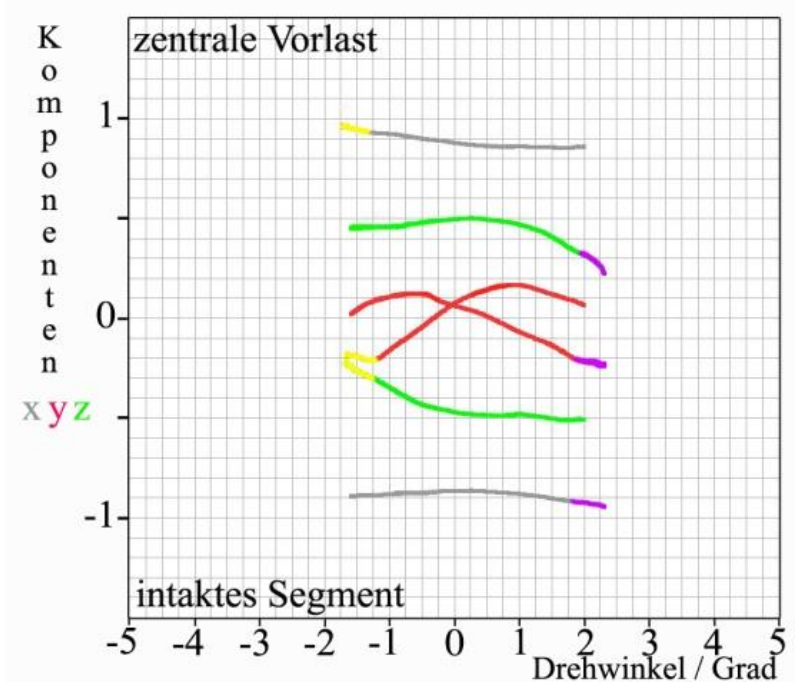

Abb. 3-41: Richtungskomponenten der Schraubachse: Lateralflexion, axiale Vorlast: $20 \mathrm{~N}$ im WZ

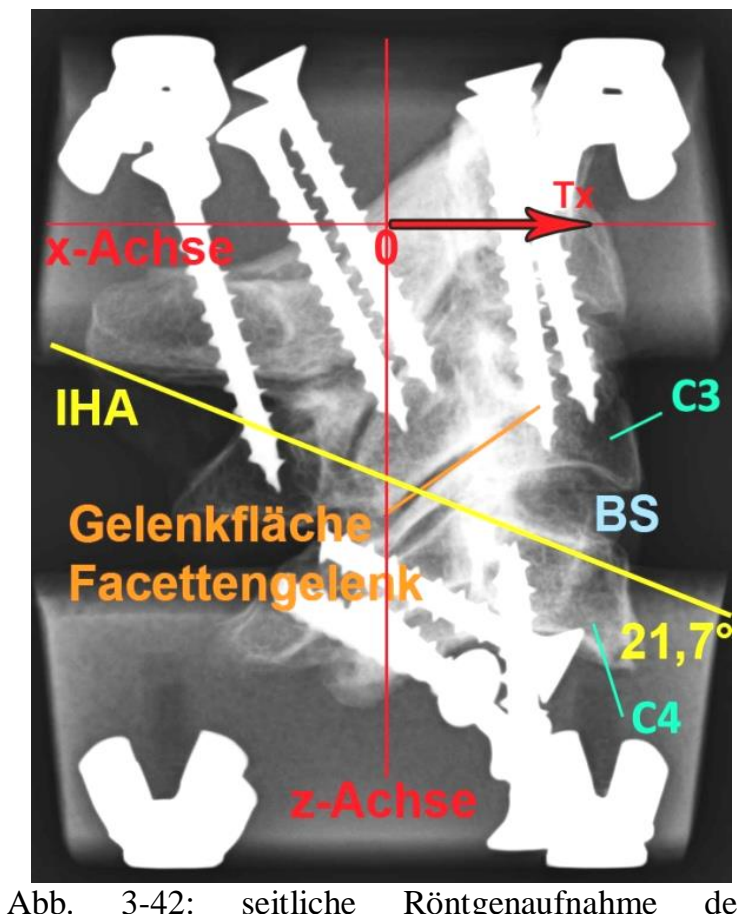

Präparates 16/07: Durchschnittliche Ausrichtung der initialen Rotationsachse bei Lateralflexion. 


\begin{tabular}{|lccc|} 
& \multicolumn{3}{c|}{ Vorlastposition } \\
\cline { 2 - 4 } & neutral & ventral & dorsal \\
\hline Vorlast $\left(\mathbf{F}_{\mathbf{z}}=\mathbf{2 0} \mathbf{N}\right)$ & 0 & $20 \mathrm{~mm}$ & $-20 \mathrm{~mm}$ \\
\hline IHA-Ausrichtung & $-68,31^{\circ}$ & $-70,15^{\circ}$ & $-68,75^{\circ}$ \\
Intaktes Segment & $\sigma=11,2^{\circ}$ & $\sigma=10,53^{\circ}$ & $\sigma=11,6^{\circ}$ \\
\hline IHA-Ausrichtung & $-64,62^{\circ}$ & $-69,84^{\circ}$ & $-61,89^{\circ}$ \\
Prestige $^{\circledR}$ LP & $\sigma=7,99^{\circ}$ & $\sigma=8,7^{\circ}$ & $\sigma=8,73^{\circ}$ \\
\hline IHA-Ausrichtung $^{\circ}$ & $-63,12^{\circ}$ & $-66,58^{\circ}$ & $-64,51^{\circ}$ \\
Bryan $^{\circledR}$ Cervical Disc & $\sigma=10,2^{\circ}$ & $\sigma=11,28^{\circ}$ & $\sigma=7,75^{\circ}$ \\
\hline
\end{tabular}

Tab. 3-13: Neigungswinkel der IHA zur Horizontalebene; Lateralflexion; Die initiale Ausrichtung der IHA wurde weder durch den Vorlastbetrag, die Vorlastposition, noch durch die Implantation der beiden Bandscheibenprothesen nennenswert verändert. Standardabweichung $(\sigma)$

\subsubsection{Drehwinkel-Drehmoment-Kennlinie, Bewegungsausmaß und Steifigkeit}

Die Drehwinkel-Drehmoment-Kennlinien wiesen eine sigmoide Form auf und zeigten eine Hysterese. Grundsätzlich waren sie mit den Alpha(T)-Kennlinien der axialen Rotation vergleichbar. Die initiale Steifigkeit des intakten Segmentes betrug unter einer zentralen Belastung von $20 \mathrm{~N}$ durchschnittlich $54,97 \mathrm{Ncm} / \mathrm{deg}$. Eine Verschiebung der Vorlast in sagittaler Richtung führte zu einer Abnahme der Steifigkeit bei ventraler Positionierung und zu einem Anstieg bei dorsaler Platzierung (Tab. 3-14). Die Implantation der Bandscheibenprothesen ging mit einer Abnahme der Steifigkeit einher, wie es bereits bei axialer Rotation beobachtet werden konnte. In Bezug auf die Entwicklung der Steifigkeit zeigte die Verlagerung der axialen Belastung im Vergleich zum intakten Segment eine gleichbleibende Tendenz. Eine Ausnahme stellte die maximal ventral positionierte Vorlast bei Prestige ${ }^{\circledR}$ LP dar, unter welcher im Unterschied zum intakten und mit Bryan ${ }^{\circledR}$ versorgten Segment eine deutliche Zunahme der Steifigkeit zu verzeichnen war. Das Ausmaß der Beweglichkeit ist in Tabelle 3-15 dargestellt. Trotz der deutlich variierenden Werten der minimalen Steifigkeit bei unterschiedlicher Lokalisation der axialen Vorlast, änderte sich das Bewegungsausmaß nur marginal.

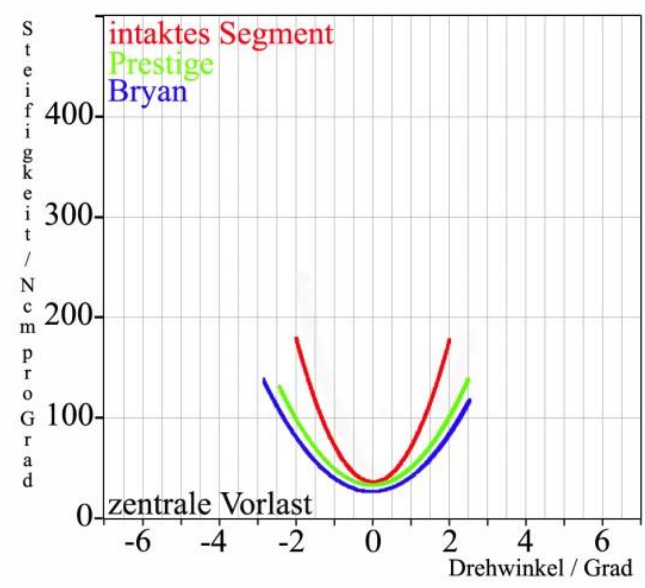

Abb. 3-43: Steifigkeitsdiagramm, Lateralflexion; Vergleich: intaktes Segment - Prestige ${ }^{\circledR}$ LP - Bryan $® ;$ Präparat 10/07: axiale Vorlast: $20 \mathrm{~N}$ im WZ. Unter Prestige ${ }^{\circledR}$ LP und Bryan ${ }^{\circledR}$ kam es zu einem leichten Abfall der Steifigkeit.

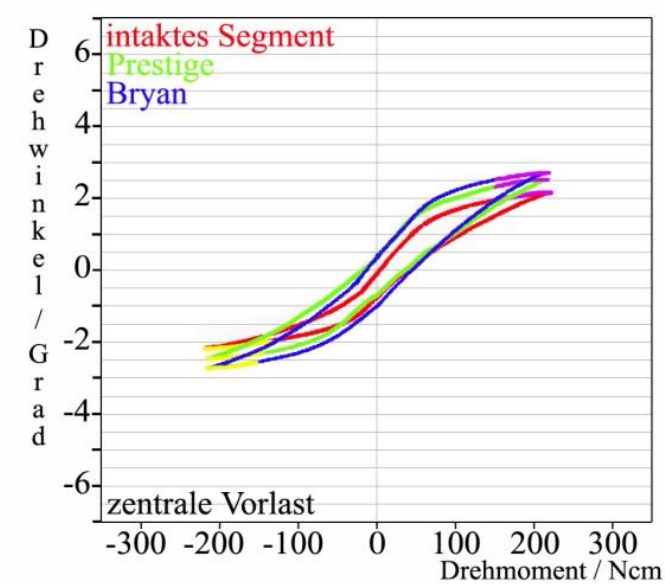

Abb. 3-44: Alpha(T)-Diagramm, Lateralflexion; Vergleich: intaktes Segment - Prestige $₫$ LP - Bryan $® ;$ Präparat 10/07; axialen Vorlast: $20 \mathrm{~N}$ im WZ. Analog zum Abfall der Steifigkeit nach Implantation der Bandscheibenprothesen kam es zu einem leichten Anstieg des Bewegungsausmaßes. 


\begin{tabular}{|c|c|c|c|c|}
\hline & & $20 \mathrm{~N}$ im WZ & $\begin{array}{l}20 \mathrm{~N} \\
20 \mathrm{~mm} \text { ventral }\end{array}$ & $20 \mathrm{~N} 20 \mathrm{~mm}$ dorsa \\
\hline \multirow[t]{2}{*}{ Intaktes Segment } & {$[\mathrm{Ncm} / \mathrm{deg}]$} & $54,97(\sigma=41,61)$ & $38,2(\sigma=22,19)$ & $70,78(\sigma=70,46)$ \\
\hline & [Ncm/deg] rel [\%] & 100 & $78(\sigma=25)$ & $166(\sigma=25)$ \\
\hline \multirow[t]{2}{*}{${\text { Prestige }{ }^{\circledR} \text { LP }}$} & {$[\mathrm{Ncm} / \mathrm{deg}]$} & $34,04(\sigma=20,8)$ & $47,05(\sigma=46,95)$ & $52,11(\sigma=40,85)$ \\
\hline & [Ncm/deg] rel [\%] & 100 & $138(\sigma=58)$ & $153(\sigma=31)$ \\
\hline \multirow{2}{*}{$\begin{array}{l}\text { Bryan }^{\circledast} \text { Cervical } \\
\text { Disc }\end{array}$} & [Ncm/deg] & $27,25(\sigma=17,96)$ & $20,34(\sigma=9,37)$ & $57,65(\sigma=47,61)$ \\
\hline & [Ncm/deg] rel [\%] & 100 & $75(\sigma=29)$ & $212(\sigma=47)$ \\
\hline
\end{tabular}

Tab. 3-14: Steifigkeit [Ncm/deg]; Vergleich zwischen intaktem Bewegungssegment, Prestige ${ }^{\circledR}$ LP und Bryan ${ }^{\circledR}$ Cervical Disc unter verschiedenen Vorlastpositionen; Lateralflexion, jeweilige Standardabweichung $(\sigma)$ in Klammern. Ähnlich der axialen Rotation war auch bei der Lateralflexion sowohl am intakten Segment, als auch bei beiden Prothesen, eine Zunahme der Steifigkeit bei dorsaler VL-Position zu beobachten. Ein Steifigkeitsabfall bei ventraler Vorlast war beim intakten Segment und bei Bryan® Cervical Disc zu sehen. Unter Prestige ${ }^{\circledR}$ LP kam es hingegen zu einer Steifigkeitszunahme unter ventraler Vorlast.

\begin{tabular}{|c|c|c|c|c|}
\hline & & $20 \mathrm{~N}$ im WZ & $20 \mathrm{~N} 20 \mathrm{~mm}$ ventral & $20 \mathrm{~N} 20 \mathrm{~mm}$ dorsal \\
\hline \multirow{2}{*}{ Intaktes Segment } & ROM [deg] & $4,11(\sigma=2,15)$ & $4,28(\sigma=2,15)$ & $4,13(\sigma=2,23)$ \\
\hline & ROM rel [\%] & 100 & $104,14(\sigma=5,73)$ & $100,49(\sigma=10)$ \\
\hline \multirow[t]{2}{*}{ Prestige ${ }^{\circledR}$ LP } & ROM [deg] & $5,25(\sigma=2,71)$ & $5,04(\sigma=2,53)$ & $5,23(\sigma=2,81)$ \\
\hline & ROM rel [\%] & 100 & $96(\sigma=5,16)$ & $99,62(\sigma=9,97)$ \\
\hline \multirow{2}{*}{$\begin{array}{l}\text { Bryan }^{\circledast} \text { Cervical } \\
\text { Disc }\end{array}$} & ROM [deg] & $5,88(\sigma=2,95)$ & $5,94(\sigma=3,01)$ & $5,26(\sigma=2,9)$ \\
\hline & ROM rel [\%] & 100 & $101,02(\sigma=9,91)$ & $89,46(\sigma=7,93)$ \\
\hline
\end{tabular}

Tab. 3-15: Range of motion (ROM); Vergleich zwischen intaktem Bewegungssegment, Prestige ${ }^{\circledR}$ LP und Bryan ${ }^{\circledR}$ Cervical Disc unter verschiedenen Vorlastpositionen; Lateralflexion, jeweilige Standardabweichung $(\sigma)$ in Klammern. Im Gegensatz zur axialen Rotation zeigte sich bei der Lateralflexion eine deutlich geringere Abhängigkeit des Bewegungsausmaßes von der Vorlastposition. Dies galt sowohl für das intakte Segment, als auch für die beiden Prothesen. Lediglich die dorsale Platzierung der Vorlast unter Bryan ${ }^{\circledR}$ Cervical Disc zeigte eine deutliche Abnahme des Bewegungsausmaßes (ca. 11\%). 


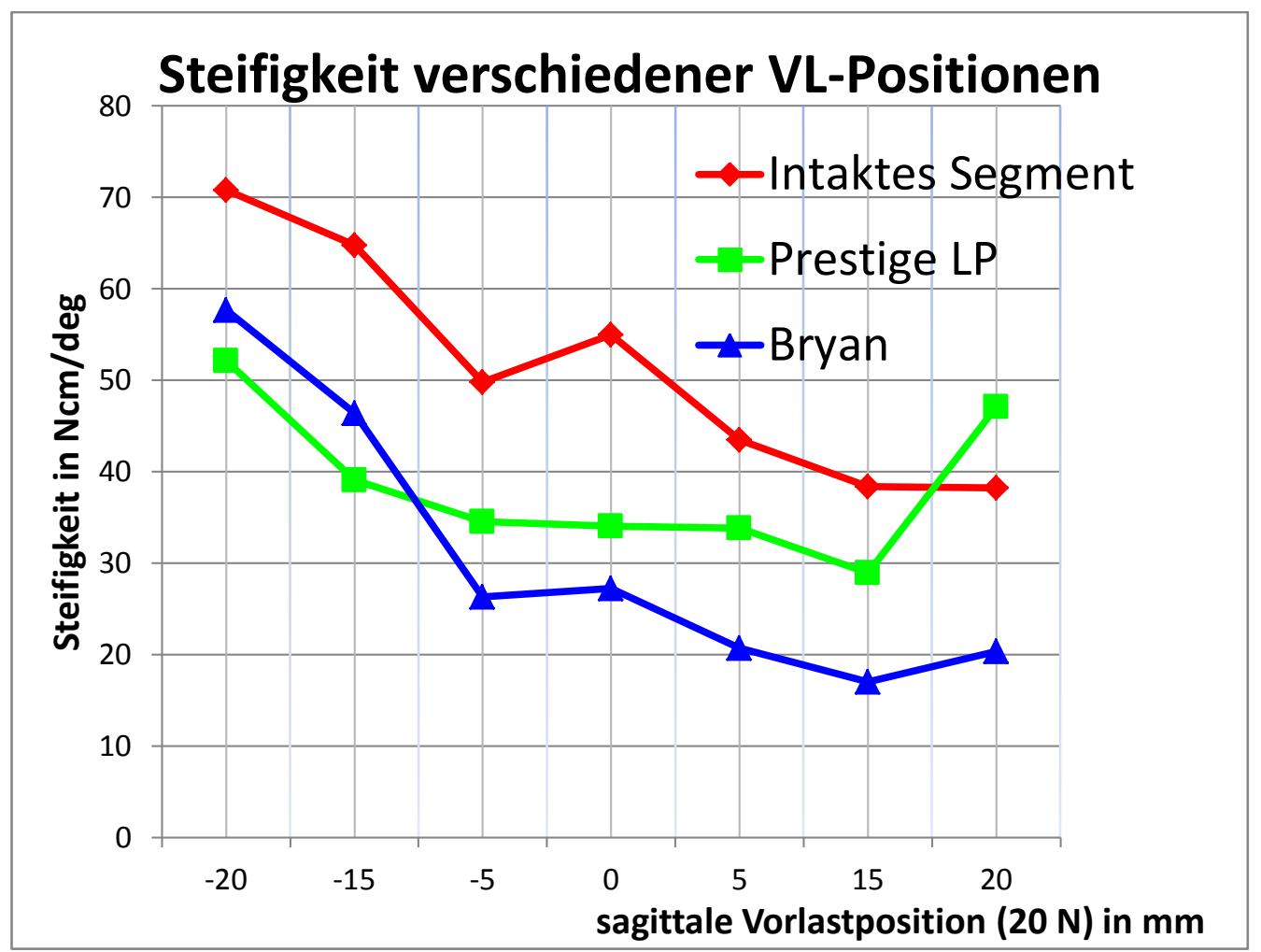

Abb. 3-45: Übersicht über die Entwicklung der initialen Steifigkeit in Abhängigkeit von der sagittalen Vorlastposition. Primär ist der allgemeine Steifigkeitsverlust nach TDA erkennbar. Die Tendenz der Steifigkeitsentwicklung in Abhängigkeit der Vorlastposition blieb nach der Prothesenimplantation erhalten. Eine Ausnahme stellte die maximal ventral positionierte Vorlast unter Prestige® LP dar, die zu einem deutlichen Anstieg der Segmentsteifigkeit führte. 


\subsection{Ventralflexion-Extension}

Die Vermessung der biomechanischen Eigenschaften bei Ventralflexion und Extension erforderten eine Anpassung der Messbedingungen. Aufgrund des hohen Bewegungsausmaßes und der geringen Segmentsteifigkeit in dieser Bewegungsrichtung, war in einzelnen Fällen eine Reduktion des applizierten Drehmomentes $T_{y}$ von 2,25 Nm auf 1,70 Nm bzw. 0,85 Nm erforderlich. Die unterschiedlichen Voraussetzungen mussten dementsprechend bei der anschließenden Datenanalyse berücksichtigt werden.

\subsubsection{Lage und Wanderung der Rotationsachse}

Die Schnittpunkte der momentanen Schraubachse mit der Median-Sagittal-Ebene bildeten die Rastpolkurven der Ventralflexion bzw. Extension. Für die acht untersuchten Präparate zeigte sich eine annähernd identische Gestalt. Verglichen mit den RPK's der axialen Rotation und Lateralflexion war ihr Erscheinungsbild weitaus weniger komplex, da nur eine geringere Wanderung stattfand. Dabei war die IHA durchgängig zwischen der oberen Deckplatte des unteren Wirbels und der Mitte des vierten Wirbelkörpers lokalisiert.

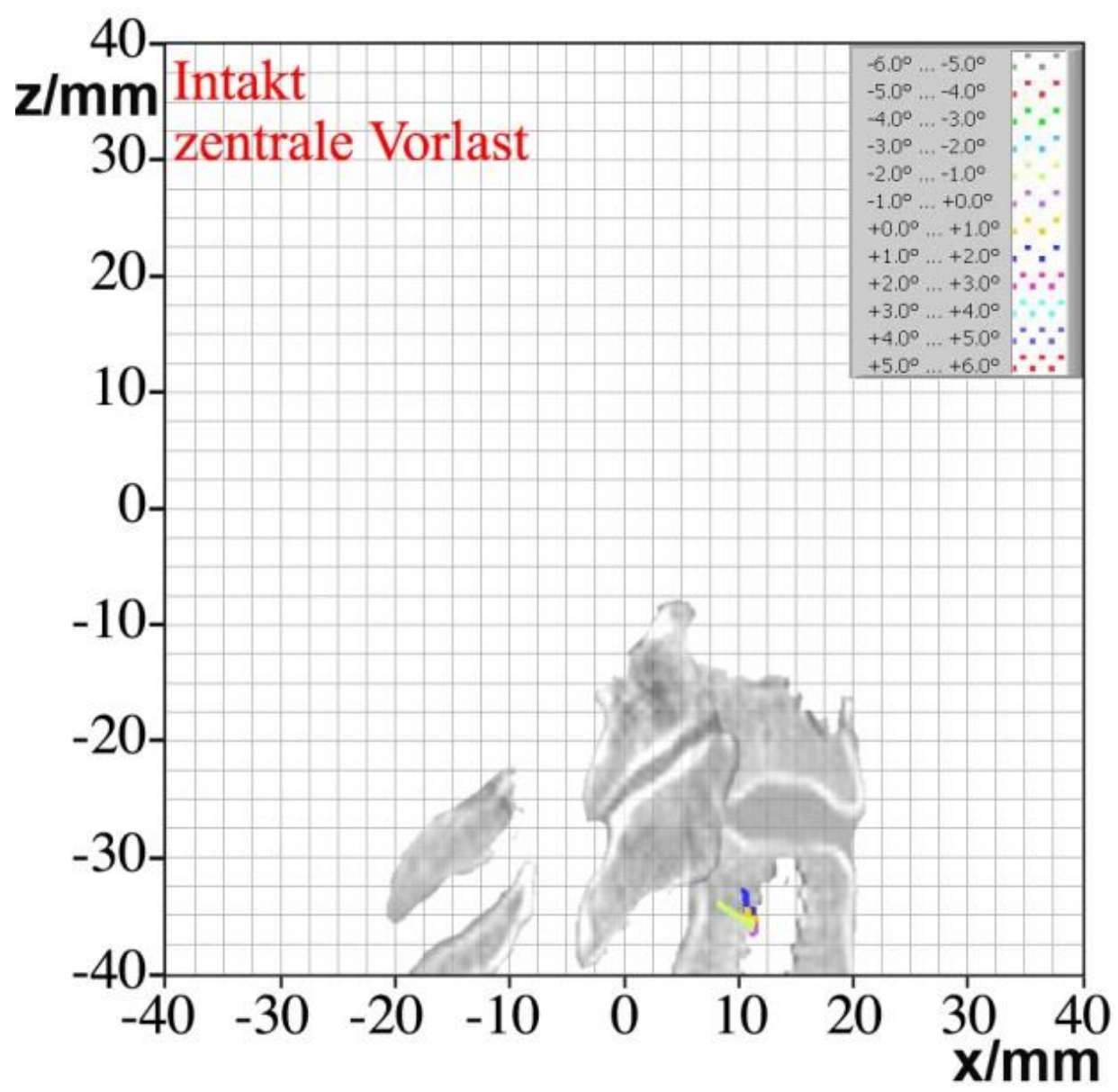

Abb. 3-46: Rastpolkurve, Ventralflexion-Extension; intaktes Segment 10/07; Vorlast: 20 N im WZ. Die IHA zeigte eine geringe Wanderung innerhalb des vierten Halswirbels. 
In maximaler Extensionsstellung (Beginn der Flexionsbewegung) lag die IHA im dorsalen Bereich des Wirbelkörpers und wanderte im Laufe der Flexion nach ventral in der Mitte des Wirbelkörpers. Dort verblieb die momentane Schraubachse in einer nahezu konstanten Position bis zur Beendigung der Vorwärtsneigung. Die Umkehr der Bewegung resultierte in einem Versatz der Achse um ca. $2 \mathrm{~mm}$ nach kranial. Im Rahmen der Extension migrierte die IHA zurück nach kaudal in die Wirbelkörpermitte. Der erneute Bewegungswechsel ging mit einem Sprung der Rotationsachse von annähernd $5 \mathrm{~mm}$ nach dorsal in die Ausgangsposition einher.

Die Erhöhung der axialen Vorlast bzw. die Änderung ihrer Position hatten keinen nennenswerten Einfluss auf das Migrationsverhalten und die Position der Rastpolkurve (Abb. 3-47).

Die Implantation der Prothesen führte analog zur Lateralflexion zu einer Verlagerung der Achsenschar um ca. $3 \mathrm{~mm}$ nach kranial. Das geringe Migrationsausmaß verblieb. Analog zur axialen Rotation zeigte sich eine dorsale Verlagerung der Rastpolkurve nach Einsatz der Bandscheibenprothesen, welcher unter ventraler Vorlast weniger stark zu beobachten war, als unter zentraler und dorsaler Belastung (Abb. 3-48). Wie auch bei der Lateralflexion kam es gleichzeitig zu einer Positionsänderung nach kranial.

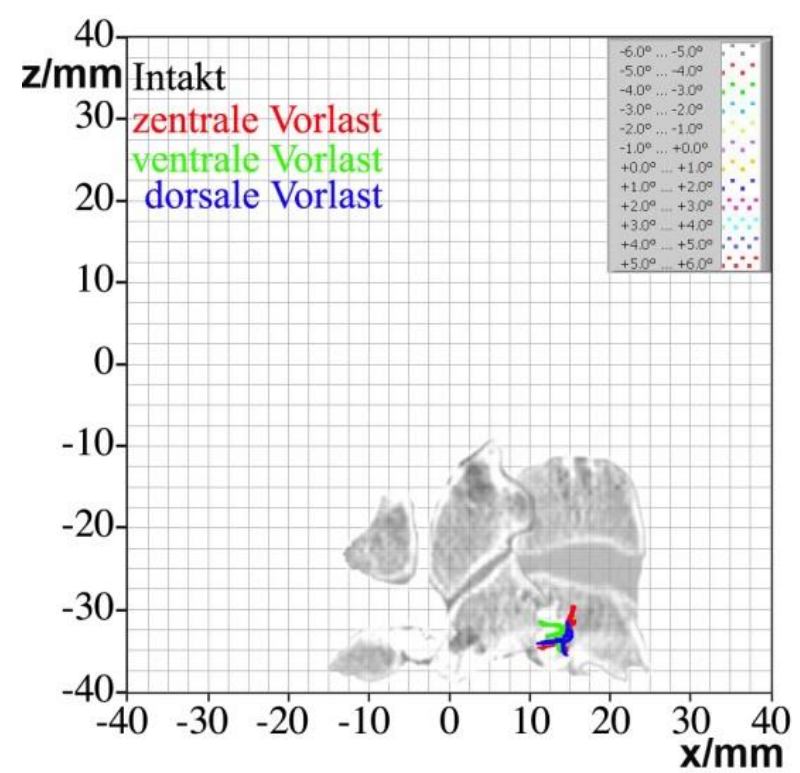

Abb. 3-47: Rastpolkurve, Ventralflexion-Extension; intaktes Segment 10/07; axiale Vorlast: 20 N 20 mm dorsal bzw. ventral und im WZ. Der Migrationsverlauf änderte sich bei unterschiedlichen Vorlastpositionen nur geringfügig.

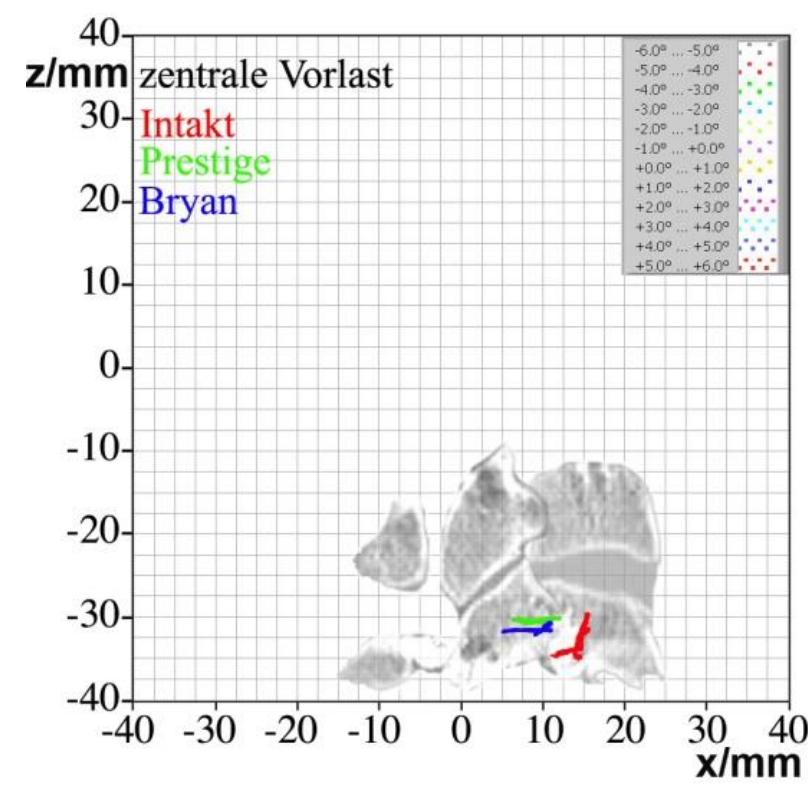

Abb. 3-48: Rastpolkurve, Ventralflexion-Extension; Präparat 10/07; axiale Vorlast: $20 \mathrm{~N}$ im WZ.

Nach Implantation der Prothesen zeigte sich ein Versatz der momentanen Schraubachse nach kranial und dorsal. Die Gestalt der RPK bleibt hingegen nach Prothesenimplantation annähernd gleich. 


\subsubsection{Ausrichtung der Rotationsachse}

Die initiale Ausrichtung der momentanen Schraubachse änderte sich im Laufe der Flexion-Extension kaum und verlief im Unterscheid zur Axialrotation und Lateralflexion annähernd parallel zum applizierten Drehmoment $\mathrm{T}_{\mathrm{y}}$ (Abb. 3-49, Abb. 3-50). Im Bereich hoher Neigungswinkel kam es vereinzelt zu einer größeren Verkippung der Achse, wobei die x-Komponente (Schwenken der IHA nach ventral bzw. dorsal) stärker betroffen war, als die z-Komponente (kraniale bzw. kaudal Verkippung).

Die Implantation der Bandscheibenprothesen und die Verlagerung der axialen Vorlast hatten lediglich einen marginalen Einfluss auf die Achsausrichtung (Tab. 3-16). Bei lateral gelegenen Vorlast verlief die IHA in der Frontalebene annähernd parallel zu der Gelenkfläche des gegenseitigen Facettengelenkes. Für linksseitige Belastung ist die IHA somit von links-dorsal und kranial nach rechts-ventral und kaudal, für rechtsseitige Vorlastposition von links-ventral und kaudal nach rechtsdorsal und kranial ausgerichtet. Dieser Ausrichtungswechsel bei lateraler Vorlastposition lässt sich auch nach Implantation der Bandscheibenprothesen nachweisen.

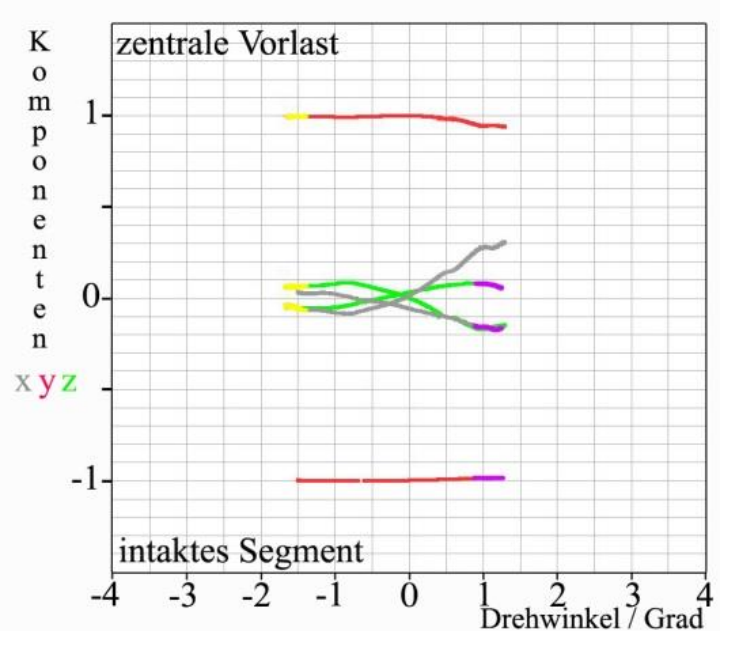

Abb. 3-49: Richtungskomponenten der Schraubachse; Ventralflexion-Extension; Vorlast: $20 \mathrm{~N}$ im WZ

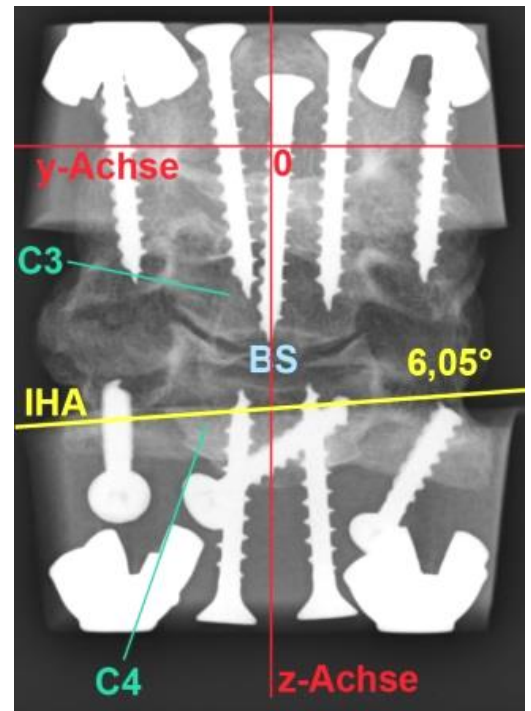

Abb. 3-50: anterior-posterior-Röntgenaufnahme des Präparates 16/07: Durchschnittliche Ausrichtung der initialen Rotationsachse in der Frontalebene bei Ventralflexion-Extension.

\begin{tabular}{|lccc|}
\hline & \multicolumn{3}{c|}{ Vorlastposition } \\
\cline { 2 - 4 } & neutral & ventral & dorsal \\
\hline Vorlast (Fz=20 N) & 0 & $20 \mathrm{~mm}$ & $-20 \mathrm{~mm}$ \\
\hline IHA-Ausrichtung & $\mathbf{8 3 , 9 5 ^ { \circ }}$ & $\mathbf{8 3 , 5 ^ { \circ }}$ & $\mathbf{8 4 , 6 2 ^ { \circ }}$ \\
Intaktes Segment & $\sigma=5,23^{\circ}$ & $\sigma=5,37^{\circ}$ & $\sigma=3,7^{\circ}$ \\
\hline IHA-Ausrichtung & $87,5^{\circ}$ & $85,12^{\circ}$ & $87,75^{\circ}$ \\
Prestige $^{\circledR}$ LP & $\sigma=1,77^{\circ}$ & $\sigma=3^{\circ}$ & $\sigma=1,28^{\circ}$ \\
\hline IHA-Ausrichtung & $\mathbf{8 7 , 2 5 ^ { \circ }}$ & $\mathbf{8 5 , 7 5 ^ { \circ }}$ & $\mathbf{8 7 , 4 3 ^ { \circ }}$ \\
Bryan $^{\circledR}$ Cervical Disc & $\sigma=1,75^{\circ}$ & $\sigma=2,66^{\circ}$ & $\sigma=0,79^{\circ}$ \\
\hline
\end{tabular}

Tab. 3-16: Neigungswinkel der IHA zur Transversalebene; Die initiale Ausrichtung der IHA wurde weder durch den Vorlastbetrag, die Vorlastposition, noch durch die Implantation der beiden Bandscheibenprothesen nennenswert verändert. (VentralflexionExtension), Standardabweichung $(\sigma)$ 


\subsubsection{Drehwinkel-Drehmoment-Kennlinie, Bewegungsausmaß und Steifigkeit}

Analog zu den Ergebnissen der axialen Rotation und Lateralflexion, zeigten sich eine sigmoide Gestalt der Drehwinkel-Drehmoment-Kennlinien und eine sichtbare Hysterese. Im Gegensatz zu den anderen Bewegungsrichtungen, führte jegliche Verschiebung der axialen Vorlast zu einer Zunahme der Steifigkeit. Dies war auch nach Prothesenimplantation erkennbar. Verglichen mit dem intakten Segment kam es trotz der Reduktion des applizierten Drehmomentes auf 0,84 Nm sowohl zu einem deutlichen Anstieg der minimalen Steifigkeit bei implantierter Prestige ${ }^{\circledR}$ LP bzw. Bryan ${ }^{\circledR}$ Cervical Disc, als auch zu einer deutlich stärkeren relativen Zunahme infolge der Vorlastverschiebung (siehe Tabelle 3-17). Analog dazu zeigte sich eine deutliche Abnahme des Bewegungsausmaßes nach der Prothesenimplantation, die bei dorsaler Vorlastposition deutlicher war, als unter ventraler Belastung (siehe Tabelle 3-18). Bei den intakten Segmenten war im Gegensatz dazu trotz Steifigkeitszunahme ein leicht erhöhtes Bewegungsausmaß nachweisbar.

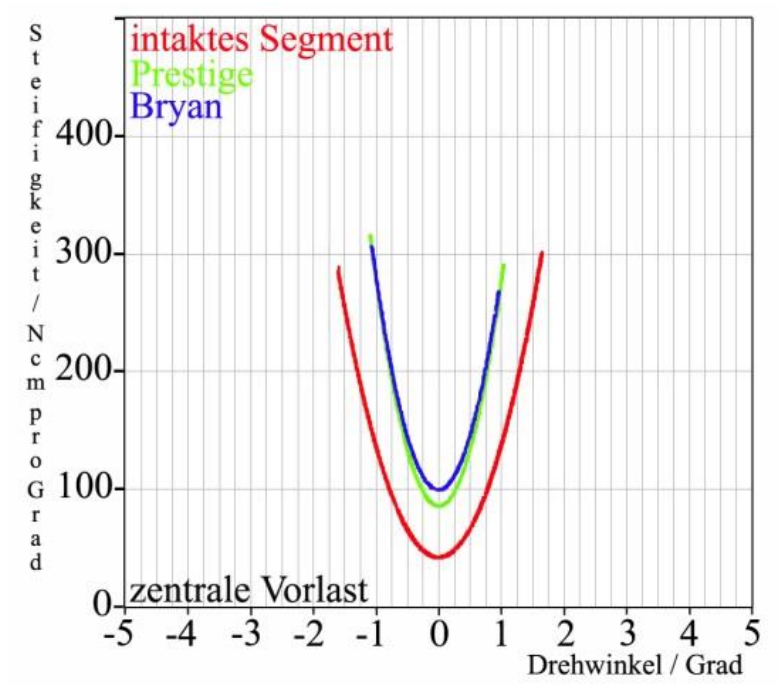

Abb. 3-51: Steifigkeitsdiagramm, VentralflexionExtension; Vergleich: intaktes Segment - Prestige ${ }^{\circledR}$ LP - Bryan®; Präparat 10/07: axiale Vorlast: 20 N im WZ. Unter Prestige ${ }^{\circledR}$ LP und Bryan ${ }^{\circledR}$ kam es zu einem Anstieg der Steifigkeit bei verringertem Drehmoment.

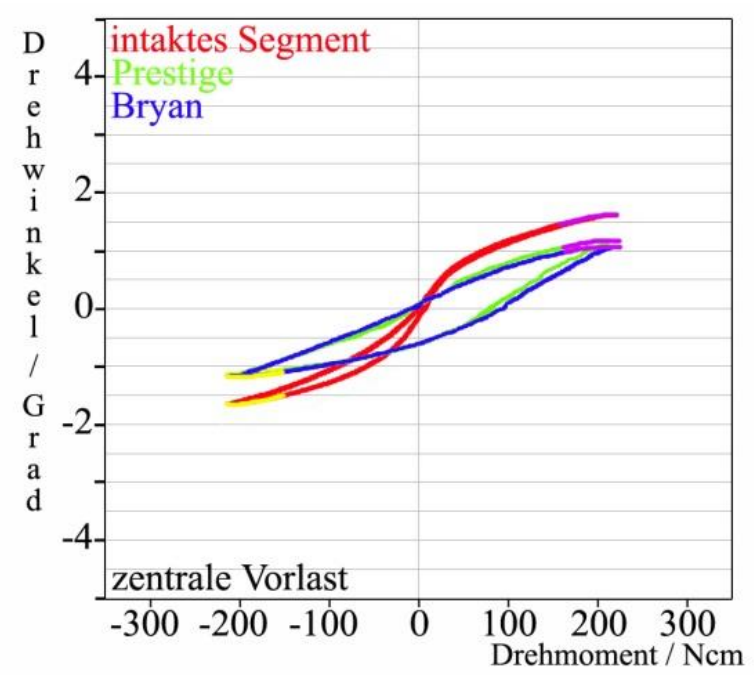

Abb. 3-52: Alpha(T)-Diagramm, VentralflexionExtension; Vergleich: intaktes Segment - Prestige ${ }^{\circledR}$ LP - Bryan®; Präparat 10/07; axialen Vorlast: 20 N im WZ. Analog zum Anstieg der Steifigkeit nach Implantation der Bandscheibenprothesen kam es zu einem Abfall des Bewegungsausmaßes bei verringertem Drehmoment. 


\begin{tabular}{|c|c|c|c|c|}
\hline & & 20N im WZ & $20 \mathrm{~N} 20 \mathrm{~mm}$ ventral & $20 \mathrm{~N} 20 \mathrm{~mm}$ dorsal \\
\hline Intaktes Segment & {$[\mathrm{Ncm} / \mathrm{deg}]$} & $37,63(\sigma=17,1)$ & $44,75(\sigma=12,04)$ & $38,75(\sigma=12,55)$ \\
\hline$T y=224 \mathrm{Ncm}$ & {$[\mathrm{Ncm} / \mathrm{deg}] \mathrm{rel}[\%]$} & 100 & $118,92(\sigma=90)$ & $102,98(\sigma=7)$ \\
\hline Prestige $^{\circledR}$ LP & [Ncm/deg] & $45,5(\sigma=29,08)$ & $70,25(\sigma=46,45)$ & $78(\sigma=39,05)$ \\
\hline$T y=84 \mathrm{Ncm}$ & [Ncm/deg] rel [\%] & 100 & $154,4(\sigma=30)$ & $171,43(\sigma=28)$ \\
\hline Bryan ${ }^{\circledR}$ Cervical & [Ncm/deg] & $49(\sigma=50,71)$ & $77(\sigma=18,09)$ & $81(\sigma=38,94)$ \\
\hline $\begin{array}{l}\text { Disc } \\
\mathrm{Ty}=84 \mathrm{Ncm}\end{array}$ & [Ncm/deg] rel [\%] & 100 & $157,14(\sigma=38)$ & $165,31(\sigma=36)$ \\
\hline
\end{tabular}

Tab. 3-17: Steifigkeit [Ncm/deg]; Vergleich zwischen intaktem Bewegungssegment, Prestige ${ }^{\circledR}$ LP und Bryan ${ }^{\circledR}$ Cervical Disc unter verschiedenen Vorlastpositionen; Ventralflexion-Extension, jeweilige Standardabweichung $(\sigma)$ in Klammern. Zu beachten ist die Reduktion des Drehmomentes nach TDA. Die absoluten Werte sind daher nicht direkt miteinander vergleichbar. Es ist lediglich ein relativer Vergleich möglich. Prozentual zeigte sich in allen Fällen eine Zunahme der Steifigkeit bei ventraler, als auch bei dorsaler VL-Position, wobei diese nach Prothesenimplantation wesentlich deutlicher war.

\begin{tabular}{|c|c|c|c|c|}
\hline & & $20 \mathrm{~N}$ im WZ & $20 \mathrm{~N} 20 \mathrm{~mm}$ ventral & $20 \mathrm{~mm}$ dorsal \\
\hline Intaktes Segment & ROM [deg] & $4,74(\sigma=1,3)$ & $4,99(\sigma=1,3)$ & $4,81(\sigma=1,2)$ \\
\hline $\mathrm{Ty}=224 \mathrm{Ncm}$ & ROM rel [\%] & 100 & $105,83(\sigma=4,03)$ & $102,40(\sigma=8)$ \\
\hline Prestige $^{\circledR}$ LP & ROM [deg] & $4,59(\sigma=1,58)$ & $3,79(\sigma=1,11)$ & $2,87(\sigma=0,79)$ \\
\hline$T y=84 \mathrm{Ncm}$ & ROM rel [\%] & 100 & $82,57(\sigma=13,33)$ & $62,53(\sigma=7,67)$ \\
\hline Bryan $^{\circledast}$ Cervical & ROM [deg] & $4,53(\sigma=1,33)$ & $3,74(\sigma=1,01)$ & $2,56(\sigma=0,51)$ \\
\hline $\begin{array}{l}\text { Disc } \\
\text { Ty= } 84 \mathrm{Ncm}\end{array}$ & ROM rel [\%] & 100 & $82,56(\sigma=19,23)$ & $56,51(\sigma=15,29)$ \\
\hline
\end{tabular}

Tab. 3-18: Range of motion (ROM); Vergleich zwischen intaktem Bewegungssegment, Prestige ${ }^{\circledR}$ LP und Bryan ${ }^{\circledR}$ Cervical Disc unter verschiedenen Vorlastpositionen bei Ventralflexion-Extension. Zu beachten ist die Reduktion des Drehmomentes nach TDA. Die absoluten Werte sind daher nicht direkt miteinander vergleichbar. Es ist lediglich ein relativer Vergleich möglich, jeweilige Standardabweichung $(\sigma)$ in Klammern. Während das Bewegungsausma $\beta$ des intakten Segmentes in alles VL-Konstellationen annähernd gleich (leichter Anstieg) blieb, zeigte sich unter Prestige ${ }^{\circledR}$ LP und Bryan ${ }^{\circledR}$ Cervical Disc eine deutlich stärkere Abhängigkeit des ROM von der Vorlastposition. Die ventrale und dorsale VL-Positionierung führten jeweils zu einer Abnahme des Bewegungsausmaßes. 


\subsection{Qualitative Beurteilung der Gelenkflächen}

Zur genaueren Analyse der Gelenkflächen erfolgte nach Abschluss aller Messreihen eine visuelle Begutachtung. Dadurch sollten die Befunde der radiologischen Untersuchung, die vor Beginn der Messungen vollzogen wurde, bestätigt werden. Es zeigte sich eine auffallende individuelle Variabilität zwischen den Präparaten bzgl. des Gelenkdurchmessers (Tabelle 3-19), aber auch hinsichtlich der Beschaffenheit der Gelenkflächen. So konnten bei den Präparaten 10/06, 11/07, 16/05 und 16/07 Unebenheiten im Bereich der artikulierenden Strukturen nachgewiesen werden, während bei den Präparaten 08/06, 10/07, 13/06 und 14/05 keine pathologischen Veränderungen erkennbar waren.

\begin{tabular}{|l|l|l|}
\hline Halswirbelpräparat & rechtes Gelenk & linkes Gelenk \\
\hline $08 / 06$ & ca. $11 \mathrm{~mm} \times 11 \mathrm{~mm}$ & ca. $10 \mathrm{~mm} \times 13 \mathrm{~mm}$ \\
\hline $10 / 06$ & ca. $17 \mathrm{~mm} \times 13 \mathrm{~mm}$ & ca. $23 \mathrm{~mm} \times 19 \mathrm{~mm}$ \\
\hline $10 / 07$ & ca. $11 \mathrm{~mm} \times 11 \mathrm{~mm}$ & ca. $10 \mathrm{~mm} \times 12 \mathrm{~mm}$ \\
\hline $11 / 07$ & ca. $17 \mathrm{~mm} \times 14 \mathrm{~mm}$ & ca. $20 \mathrm{~mm} \times 15 \mathrm{~mm}$ \\
\hline $13 / 06$ & ca. $14 \mathrm{~mm} \times 15 \mathrm{~mm}$ & ca. $14 \mathrm{~mm} \times 14 \mathrm{~mm}$ \\
\hline $14 / 05$ & ca. $14 \mathrm{~mm} \times 12 \mathrm{~mm}$ & ca. $14 \mathrm{~mm} \times 14 \mathrm{~mm}$ \\
\hline $16 / 05$ & ca. $19 \mathrm{~mm} \times 15 \mathrm{~mm}$ & ca. $20 \mathrm{~mm} \times 15 \mathrm{~mm}$ \\
\hline $16 / 07$ & ca. $20 \mathrm{~mm} \times 15 \mathrm{~mm}$ & ca. $13 \mathrm{~mm} \times 11 \mathrm{~mm}$ \\
\hline
\end{tabular}

Tab. 3-19: Übersicht über die Maße der Facettengelenksflächen der acht Präparate (Längsdurchmesser $x$ Querdurchmesser) 


\subsection{Zusammenfassung}

\section{$\underline{\text { Intaktes Segment }}$}

Das Migrationsverhalten und die Ausrichtung der momentanen Schraubachse waren während eines Bewegungszyklus stark von der Richtung des anliegenden Drehmomentes $\left(T_{x} ; T_{y} ; T_{z}\right)$ abhängig. So lag die IHA bei Ventralflexion - Extension $\left(T_{y}\right)$ nahezu ortsfest im mittleren Bereich des vierten Halswirbelkörpers, annähernd parallel zum angreifenden Drehmoment (IHA - Neigung zur Transversalebene durchschnittlich maximal $6^{\circ}$ ). Eine Änderung des Betrages oder der sagittalen Position der axialen Vorlast blieb ohne signifikanten Einfluss auf die Ausrichtung und Position der Drehachse. Jedoch führte eine Verschiebung der Vorlast in lateraler Richtung zu einer Änderung der Achsausrichtung, wodurch die Drehachse annähernd parallel zum gleichseitigen Facettengelenk verlief.

Ein deutlich ausgeprägteres Migrationsverhalten war während der Lateralflexion und axialen Rotation zu beobachten. Im Rahmen der Seitneigung $\left(T_{x}\right)$ wanderte die IHA annähernd geradlinig auf Höhe des dritten Halswirbelkörpers in seitlich-kranialer Richtung. Eine sagittale Verschiebung der Vorlast führte zudem zu einer Änderung des Migrationsverlaufes. Es zeigte sich eine seitliche Stauchung der Rastpolkurve bei ventraler Vorlastposition, wohingegen bei dorsal platzierten Vorlasten eine vermehrte Wanderung der IHA in seitlicher Richtung zu verzeichnen war.

Eine Abhängigkeit des Migrationsverhaltens vom Betrag bzw. der Position der axialen Vorlast war ebenfalls bei axialer Rotation (Drehmoment $T_{z}$ ) erkennbar. Grundsätzlich zeigte sich während der Rotationsbewegung eine bogenförmige Wanderung der momentanen Drehachse im mittleren und hinteren Bereich der Bandscheibe. Eine Erhöhung des Vorlastbetrages, sowie eine Verlagerung dieser nach dorsal führten zu einer seitlichen Stauchung und einem Versatz der gesamten Rastpolkurve nach dorsal $\left(\Delta \mathrm{x}_{\mathrm{ne}} \approx 1,08 \mathrm{~mm}\right)$. Die Verlagerung der axialen Vorlast nach ventral ging mit einer Zunahme der seitlichen Ausläufer einher, während seitliche Vorlastpositionen zu einer Verschiebung der RPK in Richtung der Vorlast führten.

Im Gegensatz zur Ventralflexion-Extension war die initiale Schraubachse $\left(\alpha=0^{\circ}\right)$ bei der Lateralflexion und axialen Rotation nicht parallel zur Richtung des entsprechenden Drehmomentes $T_{x}$ bzw. $T_{z}$ ausgerichtet, sondern um jeweils $20-30^{\circ}$ zum angreifenden Drehmoment verkippt. Sie verlief von dorsal-kranial nach ventral-kaudal und war bei der Axialrotation durchschnittlich etwa $67^{\circ}$ zur Horizontalebene geneigt. Für die Lateralflexion konnte eine Neigung der IHA zur Horizontalebene von durchschnittlich ca. $22^{\circ}$ ermittelt werden. Dabei lag die momentane Drehachse initial annähernd in der Sagittalebene. Mit zunehmendem Rotationswinkel schwenkte die IHA jedoch in lateraler Richtung aus, so dass eine kegel- bzw. fächerförmige Regelfläche entstand. 
Die Steifigkeit und das Bewegungsausmaß (ROM) des intakten Segmentes hingen ebenfalls von der Richtung des applizierten Drehmomentes ab. Bei einer zentralen Vorlast von $20 \mathrm{~N}$ galt für die initiale Steifigkeit $D: D\left(T_{y}\right)=37,63 \mathrm{Ncm} /$ deg $<D\left(T_{z}\right)=42,75 \mathrm{Ncm} /$ deg $<D\left(T_{x}\right)=54,97 \mathrm{Ncm} /$ deg. Dementsprechend ergab sich für das Bewegungsausmaß: $\operatorname{ROM}\left(T_{y}\right)=4,74$ deg $>\operatorname{ROM}\left(T_{z}\right)=4,28$ deg $>$ $\operatorname{ROM}\left(T_{x}\right)=4,11$ deg. Unabhängig von der Richtung des Drehmomentes führte eine Erhöhung der zentralen Vorlast, sowie eine Verlagerung nach dorsal zu einem Anstieg der Steifigkeit. Analog dazu war eine Abnahme des Bewegungsausmaßes zu beobachten. Wurde die Vorlast hingegen nach ventral verschoben, nahm die Steifigkeit bei Axialrotation und Lateralflexion ab, bei VentralflexionExtension jedoch zu. Damit einhergehend war eine Zunahme des ROM zu verzeichnen. Eine Vorlastverlagerung in seitlicher Richtung zeigte hingegen keinen nennenswerten Effekt auf die Steifigkeit und das Bewegungsausmaß.

\section{$\underline{\text { Vergleich des intakten Segmentes mit Prestige }{ }^{\circledR} \text { LP Cervical Disc und Bryan }{ }^{\circledR} \text { Cervical Disc }}$}

Die Implantation der Bandscheibenprothesen ging mit einer Veränderung im Migrationsverhalten der momentanen Drehachse einher. Obwohl die Kurvengestalt und die Lage der Rastpolkurven nach der Implantation sichtbar von den Ergebnissen unter physiologischen Bedingungen abwichen, bestand eine zum intakten Segment gleichbleibende Tendenz des Migrationsverhaltens in Abhängigkeit vom Betrag und der Position der axialen Vorlast und der Richtung des applizierten Drehmomentes $\left(T_{x} ; T_{y}\right.$; $\mathrm{T}_{\mathrm{z}}$ ).

Auffällig war hingegen eine deutliche Verlagerung der IHA nach dorsal. Im Vergleich zum intakten Segment kam es bei axialer Rotation unter Prestige ${ }^{\circledR}$ LP (zentrale Vorlast: 20 N) zu einem sagittalen Versatz (x-Komponente) der initialen Schraubachse in dorsaler Richtung von 5,9 mm. Der Einsatz der Bryan ${ }^{\circledR}$ Cervical Disc führte zu einem Versatz der IHA um 8,15 mm nach dorsal. Analog dazu verlagerte sich der Schwerpunkt der gesamten Rastpolkurve infolge TDA ebenfalls nach dorsal ( $x$ Komponente) ( $x_{s}$ (intakt-prestige) $=-4,39 \mathrm{~mm} ; \mathrm{x}_{s}$ (intakt-bryan) $=-6,51 \mathrm{~mm}$ ).

Mit der Implantation der Prothesen zeigte sich zudem eine stärkere Abhängigkeit der Lage der momentanen Schraubachse von der sagittalen Vorlastposition. Die y-Komponente des entsprechenden Verschiebungs-Vektors blieb dabei jeweils vernachlässigbar klein. Bei Axialrotation hatte der Grad der Flexions- bzw. Extensionsstellung vor Rotationsbeginn im intakten Segment keinen nennenswerten Einfluss auf die Verlagerung (Versatz der zentralen RPK zwischen maximal flektierter und extendierter Ausgangslage: $\Delta \mathrm{x}_{\mathrm{fe}}($ intakt)= 1,56 mm). Mit Implantation der Prothesen stieg die RPK-Verschiebung teilweise auf das Vierfache an (Prestige ${ }^{\circledR}$ LP: $\Delta \mathrm{x}_{\mathrm{fe}}$ (prestige)= 5,63 mm; Bryan $^{\circledR}: \Delta \mathrm{x}_{\mathrm{fe}}($ bryan) $=6.33 \mathrm{~mm})$. Gleichermaßen war eine signifikante Veränderung der initialen Migrationsrate (IMR) zu beobachten. Speziell bei dem Vergleich des intakten Segmentes mit Bryan ${ }^{\circledR}$ Cervical Disc System wurde ein signifikanter Unterschied erkennbar $\left(\left\langle\Delta \mathrm{v}_{\mathrm{ybi}}\right\rangle=2.33 \mathrm{~mm} / \mathrm{deg}, \mathrm{SD}=5,38\right.$ 
$\mathrm{mm} / \mathrm{deg}$, student t-test: $d f=47, p<0.01)$. Die Gegenüberstellung beider Prothesen $\left(\Delta v_{y b p}=v_{y, b r y a n}-\right.$ $\left.v_{y, \text { prestige }}\right)$ zeigte ebenfalls eine signifikante Differenz $\left(\left\langle\Delta v_{y b p}\right\rangle=1.47 \mathrm{~mm} / \mathrm{deg}\right.$, student t-test: $d f=47$, $\mathrm{p}=0,057)$ ). Die Abweichung der IMR zwischen intaktem Segment und Prestige LP ${ }^{\circledR}$ blieb hingegen ohne Signifikanz $\left(<\Delta v_{y p i}\right\rangle=0.89 \mathrm{~mm} / \mathrm{deg}$, student test: $\mathrm{df}=47$, n.s. $)$.

Die Ausrichtung der momentanen Schraubachse änderte sich in allen drei Bewegungsrichtungen nach der Prothesenimplantation kaum. Die Neigung der Schraubachse in der Sagittalebene blieb bei axialer Rotation nach der Implantation der Bandscheibenprothesen mit $\approx-69^{\circ}$ nahezu konstant (intaktes Segment $\approx-67^{\circ}$ ) und war weiterhin unabhängig von Betrag und Position der axialen Vorlast. Bei der Lateralflexion nahm die initiale Neigung der IHA zur Horizontalebene von ca. $22^{\circ}$ (intaktes Segment) auf etwa $26^{\circ}$ nach TDA zu, während sie bei Ventralflexion-Extension weiterhin nahezu parallel zum applizierten Drehmoment $\mathrm{T}_{\mathrm{y}}$ (Verkippung ca. $3^{\circ}$ ) verlief.

Die Implantation der Bandscheibenprothesen hatte einen deutlichen Anstieg des Bewegungsausmaßes (ROM) zur Folge. Bei gleichbleibendem Drehmoment $(2,25 \mathrm{Nm})$ und zentraler

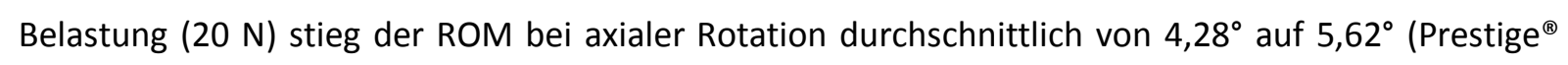
LP) bzw. $6,08^{\circ}$ (Bryan ${ }^{\circledR}$ ). Simultan kam es zu einer deutlichen Reduktion der minimalen Steifigkeit um 36\% (Prestige ${ }^{\circledR}$ LP) bzw. 45\% (Bryan ${ }^{\circledast}$ ). Der Vergleich der relativen Steifigkeit mit dem intakten Segment zeigte bei Prestige ${ }^{\circledR}$ LP eine annähernd gleichbleibende und von der Vorlastposition unabhängige Abnahme der Segmentstabilität. Hingegen war nach der Implantation der Bryan ${ }^{\circledR}$ Cervical Disc ein deutlich höherer Effekt auf die Segmentsteifigkeit infolge einer Vorlastverlagerung zu beobachten.

Bei Lateralflexion ging die Implantation der Bandscheibenprothesen mit einer Abnahme der Steifigkeit einher, wie es bereits bei axialer Rotation nachgewiesen werden konnte. In Bezug auf die Entwicklung der Steifigkeit zeigte die Verlagerung der axialen Belastung eine gleichbleibende Tendenz. Eine Ausnahme stellte die ventrale Vorlastposition bei Prestige $\mathrm{LP}^{\circledR}$ dar, unter welcher die Steifigkeit deutlich zunahm. Das Bewegungsausmaß änderte sich trotz der deutlich variierenden Werten der minimalen Steifigkeit bei unterschiedlicher Lokalisation nur marginal.

Aufgrund des hohen Bewegungsausmaßes und der geringen Segmentsteifigkeit bei der Ventralflexion-Extension, war nach TDA eine Reduktion des applizierten Drehmomentes $T_{y}$ von 2,25 $\mathrm{Nm}$ auf 1,70 Nm bzw. 0,85 Nm erforderlich. Verglichen mit dem intakten Segment kam es trotz der Reduktion des applizierten Drehmomentes sowohl zu einem deutlichen Anstieg der minimalen Steifigkeit nach TDA, als auch zu einer deutlich stärkeren relativen Zunahme infolge der Vorlastverschiebung. Analog dazu zeigte sich eine deutliche Abnahme des Bewegungsausmaßes. 


\section{Diskussion}

\subsection{Bewegungsstruktur des Halswirbelsäulensegmentes C3/C4}

In der vorliegenden Studie gelang es, genaue Daten über Lage und Wanderung der momentanen Schraubachse (IHA) nach Implantation von zwei verschiedenen Bandscheibenprothesen („Total disc arthroplasty“; TDA) im Halswirbelbereich (Segment C3/C4) zu erheben und somit Aussagen über die kinematischen Eigenschaften des Segmentes zu machen. Verwendet wurden die Prestige ${ }^{\circledR}$ LP und Bryan ${ }^{\circledR}$ Cervical Disc der Firma Medtronic Sofamor Danek. Untersucht wurden die biomechanischen Eigenschaften von acht humanen Präparaten, deren Vermessung in den drei Bewegungsrichtungen Ventralflexion-Extension, Lateralflexion sowie axiale Rotation erfolgte. Zunächst fanden die Experimente unter physiologischen Bedingungen statt, um Veränderungen nach TDA besser einordnen und bewerten zu können. Unter der Applikation verschiedener axialer Vorlasten in unterschiedlichen Positionen und der Anlage eines zyklischen, dreiecksförmigen Drehmomentes $T_{(x, y, z)}$ an den Halswirbel C3 wurden dessen Positionen gegenüber dem fixierten vierten Halswirbel aufgezeichnet. Pro Zyklus wurden ca. 400 Positionen des Halswirbels mit einer Auflösung von <0,01 für die Änderung der Rotation, sowie von $<2,4 \mu \mathrm{m}$ für die Änderung der Translation registriert. Damit gelang es in dieser Arbeit aufeinander folgende Positionsänderungen, die weniger als ein Vierhundertstel der maximalen Positionsänderung in einem Zyklus betrugen, zu erfassen. Mit dieser Genauigkeit war es möglich den Migrationsweg und die Ausrichtung der momentanen Schraubachse (IHA; „Instantaneous Helical Axis") in hinreichender Näherung zu ermitteln. Damit ergaben sich auch die allgemeinen biomechanischen Eigenschaften des Segments: Steifigkeit und Bewegungsausmaß (ROM; „Range of Motion“).

Das Wissen über die Kinematik der Wirbelsäule ist zurzeit noch sehr mangelhaft. Nur wenige Studien befassen sich mit der Position und Ausrichtung der segmentalen Drehachse (Haberl et al. 2004, Niosi et al. 2006, Rousseau et al. 2006, Galbusera et al. 2008b, Wachowski et al. 2009a, Lazaro et al. 2010, Kowalczyk et al. 2011). Die Berechnungen zur Rotationsachse sind in diesen Studien allerdings nicht präzise genug, da lediglich eine geringe Anzahl an Positionen der Schraubachse während eines Bewegungszyklus berechnet wurde. So ging man in zahlreichen Publikationen von einer ortsfesten "helical axis of motion" (HAM) aus, um die der bewegte Wirbelkörper für das gesamte Bewegungsausmaß (ROM) eine schraubenförmige Bewegung ausführe. Die Berechnung der HAM erfordert dabei lediglich zwei Positionen des bewegten Körpers, deren Unterschied etwa dem ROM entspricht (Cossette et al. 1971, Haberl et al. 2004, Niosi et al. 2006, Oxland et al. 1994, Panjabi et al. 1994, White und Panjabi 1978, Zhu et al. 2007). Die geringe Auflösung veranlasste andere Arbeitsgruppen dazu den ROM in mehrere Intervalle zu unterteilten und für jeden Abschnitt die Schraubachse („Finite Helical Axis“; FHA) zu berechnen (Kettler et al. 2004, Rousseau et al. 2006). Die 
HAM und FHA spiegeln Mittelwerte wider. Eine Beschreibung der tatsächlichen Bewegungsvorgänge des Segments ist damit nicht möglich, da sie für diese von vornherein stationäre Schraubachsen voraussetzen, um die das Segment schrauben würde.

Die Datenlage über die Kinematik infolge eines Bandscheibenersatzes (TDA; „Total Disc Arthroplasty") ist noch schlechter. Zwar gibt es Berechnungen zum Bewegungsausmaß und der HAM für Flexions-Extensions-Bewegungen anhand von einzelnen in-vitro und in-vivo Studien, jedoch liegen keine Daten vor, welche den Anforderungen einer differentiellen Auflösung entsprechen würden (Patwardhan et al. 2010, Lazaro et al. 2010, Kowalczyk et al. 2011). Die meisten Ergebnisse aus invivo Studien beschränken sich auf Flexions-Extensions-Bewegungen und die Berechnung des Rotationszentrums (COR; "Center Of Rotation“) anhand zweier Punkte im seitlichen Röntgenbild. Dies reicht aus physikalischer Sicht jedoch nicht aus, um valide Aussagen über die Kinematik machen zu können. Bei einer stationären IHA könnte das COR, trotz physikalisch inkorrektem Ansatz, näherungsweise die Position der momentanen Drehachse widerspiegeln. Doch dieser stationäre Charakter müsste erst durch hinreichend genaue Messungen belegt werden. Liegt hingegen eine ausgeprägte Migration der momentanen Schraubachse vor, wie es bei axialer Rotation, Lateralflexion und im Falle der Extension-Flexion bei ausgeprägter Extension vorkommt, spiegelt die Berechnung des COR und der HAM nicht die Wirklichkeit wider. Da bislang kaum Standards für die Erforschung der Biomechanik der Wirbelsäule vorliegen, arbeiten viele Gruppen immer noch mit nichtkinematischen Größen wie ROM, Steifigkeit und Verformung. Im Jahre 2008 bekräftigte Galbusera et al., dass es keinerlei Studien gibt, welche eine Aussage über die IHA nach totalem Bandscheibenersatz treffen. Die Kinematik bewegungserhaltender Implantate kann durch die Beurteilung des Bewegungsausmaßes (ROM) nicht hinreichend beschrieben werden (Kettler et al. 2004, Rousseau et al. 2006, Schmidt et al. 2009, Galbusera et al. 2008b, McAfee et al. 2006, Wachowski 2009a). In der vorhandenen Literatur gibt es zudem nur Spekulationen, welche Parameter zur genaueren Charakterisierung geeignet sind (Cheng und Welch 2011). Physikalisch korrekt definierte, kinematische Merkmale werden durch die Position und Ausrichtung der momentanen Schraubachse charakterisiert und sind daher zentrale Parameter in dieser Studie.

\subsubsection{Axiale Rotation}

\section{Ausrichtung der momentanen Schraubachse}

In keiner der durchgeführten Messungen, weder am intakten Segment, noch bei Prestige ${ }^{\circledR}$ LP und Bryan $^{\circledast}$ Cervical Disc, ist die Ausrichtung der momentanen Rotationachse gleich der Richtung des applizierten Drehmomentes $\left(T_{z}\right)$. Sie liegt um ca. $23^{\circ}$ nach dorsal geneigt annähernd in der Sagittalebene und demzufolge nahezu perpendikular zu den Gelenkflächen der Wirbelbogengelenke. 
Dadurch ist bei jeder axialen Rotationsbewegung gleichzeitig eine Bewegung in lateraler Richtung (Lateralflexion) zu beobachten (Kalscheuer 2001, Wachowski et al. 2007, Wachowski et al. 2009a, Patwardhan et al. 2010). Frühere Studien zeigen, dass die Resektion der Facettengelenke zu einer parallelen Ausrichtung der IHA und des axialen Drehmomentes führt (Wachowski et al. 2007). Die Ausrichtung der Rotationsachse ist demzufolge von der Gelenkführung der Facettengelenke abhängig. Folglich kommt es weder durch Prestige ${ }^{\circledast}$ LP $(\Delta \mathrm{HHA}-$ Ausrichtung= Änderung der IHARichtung im Vergleich zum intakten Segment: maximal $\left.3^{\circ}\right)$, noch durch Bryan ${ }^{\circledR}$ Cervical Disc $(\Delta \mathrm{IHA}$ Ausrichtung maximal: $4^{\circ}$ ) zu einer signifikanten Veränderung der Ausrichtung der jeweiligen Schraubachsen, solange die Funktion der Wirbelbogengelenke erhalten bleibt. Die Verlagerung der axialen Vorlast hat ebenfalls nur einen marginalen Effekt auf die Achsausrichtung. So zeigt sich für das intakte Segment ein maximaler Unterschied von $1,2^{\circ}$ in den verschiedenen Vorlastpositionen. Unter Prestige ${ }^{\circledR}$ LP kommt es zu einer maximalen Änderung von $4,8^{\circ}$ und bei Bryan ${ }^{\circledR}$ Cervical Disc von $1,8^{\circ}$. Dieser Befund scheint im ersten Moment etwas widersprüchlich, da die Gelenkflächen der Wirbelbogengelenke in Flexionsstellung theoretisch ihren Kontakt verlieren könnten und die Führung durch die Facettengelenke dadurch aufgehoben werden würde. Während der Rechts- bzw. Linksrotation (axiales Drehmoment $\mathrm{T}_{\mathrm{z}}(\mathrm{t})$ ) scheint es jedoch zu einem erneuten Kraftschluss des rechten bzw. linken Facettengelenkes zu kommen und eine Ein-Gelenk-Führung zu entstehen, wie sie im Bereich der Lendenwirbelsäule auftritt (Nägerl et al. 1995, Mansour et al. 2004, Wachowski et al. 2009a, Wachowski et al. 2009b, Wachowski et al. 2010). Im Bereich kleiner, physiologischer Rotationswinkel ( $\alpha \approx \pm 1$ Grad) finden sich sowohl vor, als auch nach der Implantation der Bandscheibenprothesen Rotationsachsen mit annähernd konstanter Ausrichtung. Dadurch kann postuliert werden, dass in diesem Rotationsintervall alle Präparate eine gleichartig ebene Bewegung ausführen. Es ist somit grundsätzlich davon auszugehen, dass die physiologische Achsausrichtung trotz Bandscheibenersatz weiterhin eingenommen werden kann und die ursprüngliche Bewegungsrichtung nahezu erhalten bleibt.

\section{Position der momentanen Schraubachse und Schwerpunkt der Rastpolkurve}

Bei allen Präparaten kann im Rahmen eines Bewegungszyklus eine deutliche Migration der Rotationsachse beobachtet werden. Während der gesamten Bewegung befindet sich die momentane Schraubachse im Bereich der mittleren bzw. hinteren Bandscheibe und zeigt bei jedem der acht Präparate eine individuelle Wanderung, welche durch die Prothesenimplantation zwar modifiziert wird, insgesamt aber eine gleichbleibende, von der Vorlast abhängige, Tendenz behält. Das Migrationsverhalten der IHA wird dabei hauptsächlich durch die Art und Anzahl der in Kontakt tretenden Gelenke beeinflusst. Treten während eines Bewegungsablaufes Gelenkflächen in Kontakt, wird die Bewegung fortan durch die entstandenen Kontaktpunkte geführt und aufgrund dessen die 
Bewegungsqualität maßgeblich definiert (Nägerl et al. 1995, Mansour et al. 2004, Wachowski et al. 2010). Dabei spielen die Wirbelbogengelenke eine entscheidende Rolle, deren vollständige Resektion zu einer Abnahme der Wanderung besonders in laterale Richtung führt, was sich folglich in einer Zentralisation der Schraubachsenposition widerspiegelt. Die IHA liegt dann, vergleichbar mit einem Bandscheibenmodell, annähernd zentral in der Bandscheibe (Kalscheuer 2001, Mansour 2004, Wachowski 2005, Hawellek 2008). Die individuelle Form und Beschaffenheit der Gelenkflächen führt zur Ausbildung unterschiedlicher Kontaktpunkte bei Kraftschluss und sorgt für das einzigartige Migrationsverhalten eines jeden Präparates. Im Bereich hoher Rotationswinkel wird die Wanderung der momentanen Schraubachse zusätzlich durch die zervikalen Uncovertebralgelenke modifiziert, die infolge des Kraftschlusses der Gelenkflächen eine übermäßige Migration in lateraler Richtung zu verhindern scheinen (Wachowski 2005, Wachowski et al. 2007, Wachowski et al. 2013).

Die Verschiebung der axialen Vorlast zeigte in der vorliegenden Studie einen deutlichen Effekt auf das Migrationsverhalten der Drehachse. Eine Belastung ventral des Widerstandszentrums äußerte sich im Allgemeinen in einer stärkeren Exkursion nach lateral in Richtung der Facettengelenke, während eine dorsal angebrachte Vorlast zu zentraleren Positionen im Wirbelkanal führte. Dieses Phänomen kann ebenfalls auf die jeweilige Gelenkführung zurückgeführt werden. Die Flexionsstellung eines Wirbels (ventrale Vorlast) kann vor allem in Neutralstellung eine Entkopplung der Wirbelbogengelenke herbeiführen (Wachowski et al. 2009b, Wachowski et al. 2010). Unter dieser Voraussetzung wäre eine später eintretende Gelenkführung durch die Facetten- oder Uncovertebralgelenke denkbar, die lediglich im Bereich größerer Rotationwinkel und mit veränderten Kontaktpunkten auftritt (Wanderung der IHA in Richtung des Gelenkes), bzw. eine zwischenzeitig vollständige Aufhebung des Kraftschlusses entstehen ließe (IHA zentral im Wirbelkörper, vgl. Bandscheibenmodell) (Mansour 2001, Wachowski 2005, Hawellek 2008).

Der Betrag der insgesamt zurückgelegten Migrationsstrecke während eines Bewegungszyklus änderte sich durch die ventrale Vorlastverlagerung letztendlich nicht. Mit durchschnittlich 47,6 mm blieb sie im Vergleich zu den Ergebnissen unter zentraler Belastung konstant. Es ist jedoch eine geringere Wanderung im Bereich kleiner Rotationswinkel $\left(\alpha \approx \pm 1^{\circ}\right)$ zu beobachten. Dies könnte dadurch erklärt werden, dass ein Kraftschluss der Facettengelenkflächen in diesen Winkelbereichen noch nicht vorliegt und eine Führung der Bewegung durch die Wirbelbogengelenke somit ausbleibt. Es könnten Verhältnisse wie bei einem Bandscheibenmodell vorliegen (IHA zentral in der Bandscheibe). Die vermehrte Migration bei hohen Auslenkwinkeln ( $\alpha$ zwischen $\pm 1^{\circ}$ und $\pm 2,4^{\circ}$ ) lässt eine zunehmende Bewegungsführung durch die Facettengelenke vermuten, welche sich erneut in einer Wanderung der IHA in Richtung des jeweils führenden Gelenkes äußert (vergleiche 3.3 „Probemessung - Bandscheibenmodell“ mit und ohne Gelenkimitation). 
Dorsal platzierte Kräfte resultieren in einer Extensionsstellung des oberen Wirbels und können einen früheren Kraftschluss (veränderte Kontaktpunkte) der Gelenkflächen zur Folge haben. Korrelierend damit war die momentane Schraubachse insgesamt nach dorsal versetzt und wanderte eher in Richtung des führenden Kontaktpunktes. Gleichzeitig werden die Freiheitsgrade des Segmentes durch zusätzliche Gelenkkontakte frühzeitig reduziert und die Beweglichkeit insgesamt eingeschränkt (Nägerl et al. 1995, Nägerl et al. 2009). Dies spiegelt sich ebenfalls in der zurückgelegten Migrationsstrecke wider, die mit ca. $39 \mathrm{~mm}$ pro Bewegungszyklus deutlich unter dem Betrag bei zentraler bzw. ventraler Vorlast lag. Allerdings ließ sich eine erhöhte Wanderung in den kleinen Rotationsbereichen $\left(\alpha \approx \pm 1^{\circ}\right)$ feststellen, die möglicherweise infolge des frühzeitigen Kraftschlusses der Gelenkflächen und der damit verbundenen Bewegungsführung entsteht.

Infolge der Prothesenimplantation zeigte sich ein signifikanter, annähernd paralleler Versatz der momentanen Drehachse nach dorsal, der eine Änderung der segmentalen Kinematik widerspiegelt. Initial $\left(\alpha=0^{\circ}\right)$ verlagerte sich die IHA unter Prestige ${ }^{\circledR}$ LP durchschnittlich um $\Delta x=-5,9 \mathrm{~mm}$, sowie um $\Delta \mathrm{x}=-8,15 \mathrm{~mm}$ mit Bryan ${ }^{\circledR}$ Cervical Disc nach dorsal, was neben der jeweils implantierten Prothese vermutlich auch zu einem gewissen Anteil auf die Strukturveränderungen im Rahmen der chirurgischen Intervention zurückzuführen ist. Die Durchtrennung der Längsbänder und die Resektion der Bandscheibe gingen in erster Linie mit einem deutlichen Verlust der Segmentsteifigkeit einher, was jedoch die Verlagerung der IHA nicht erklären kann. Vielmehr ist von veränderten Verhältnissen im Bereich der Gelenkführung auszugehen. Infolge der Teilresektion der Uncovertebralgelenke bei der Implantation der Bandscheibenprothesen wird deren Funktionsweise maßgeblich beeinflusst (Entkopplung der Gelenkflächen) (Wachowski et al. 2007) und das Gleichgewicht im Zusammenspiel mit den Wirbelbogengelenken (siehe 1.5.5 Biomechanische Eigenschaften intakter Segmente) gestört. Aufgrund dessen ist die Entstehung neuer Kontaktpunkte der Facettengelenksflächen denkbar. Als weitere Ursache wäre eine neu entstandene Extensionsstellung des dritten Halswirbels aufgrund der Prothesenimplantation denkbar. Da eine Dorsalverlagerung der IHA bereits beim intakten Segment (dorsale Vorlastposition) erkennbar ist, scheint die Prä-Extension des oberen Wirbels ein ausschlaggebender Impuls für den Versatz der Rotationsachse zu sein. Aufgrund dieser Erkenntnis und um eine Verfälschung der Messergebnisse zu vermeiden, wurde während der Implantation der Bandscheibenprothesen besonders auf die Stellung der Wirbel zueinander geachtet und deren Lage am Ende des Eingriffes kontrolliert, so dass dieser Mechanismus unwahrscheinlich erscheint (Abb. 4-1). Welcher der beiden Mechanismen letztendlich greift ist nicht eindeutig zu belegen. 


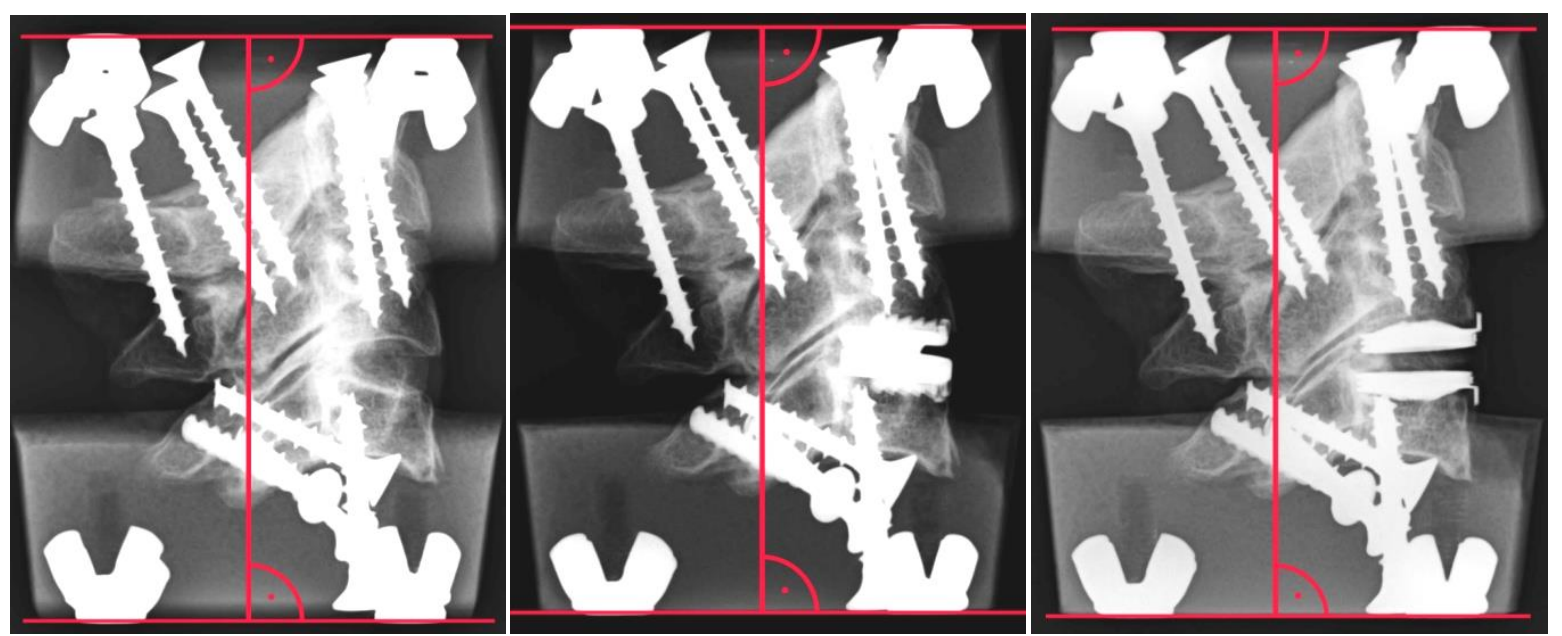

Abb. 4-1: Position des C3-Wirbels vor und nach TDA; seitliche Röntgenaufnahmen Präparat 16/07; Die Kunststoffblöcke stehen sowohl vor, als auch nach Prothesenimplantation parallel zu einander.

Im Gegensatz zum intakten Segment zeigt die Position der momentanen Schraubachse zudem eine verstärkte Abhängigkeit von der sagittalen Vorlastverschiebung. Die Differenz der Drehachsenposition zwischen ventraler und dorsaler Belastung war bei Prestige ${ }^{\circledR}$ LP $\left(\Delta \mathrm{x}_{\mathrm{fe}}\right.$ (prestige $)=$ $5,63 \mathrm{~mm})$ und Bryan ${ }^{\circledR}$ Cervical Disc $\left(\Delta \mathrm{x}_{\mathrm{fe}}(\right.$ bryan $\left.)=6,33 \mathrm{~mm}\right)$ signifikant höher als im intakten Zustand $\left(\Delta \mathrm{x}_{\mathrm{fe}}(\right.$ intakt $\left.)=1,56 \mathrm{~mm}\right)$ (siehe Tabelle 3-8). Die Resektion der Ligamenta longitudinalia, sowie des Discus intervertebralis resultierte in einer erhöhten Labilität des Segmentes für Extensions- und Flexionsbewegungen. Dadurch verstärkte sich die Prä-Flexion bzw. Prä-Extension des dritten Halswirbels bei entsprechender Vorlastposition, was wiederum andere Gelenkkontaktpunkte generiert und damit den größeren Versatz der IHA verursacht. Die erhebliche Parallelverschiebung der Rotationsachse führt letztendlich zu einer zusätzlichen Translation des beweglichen Segmentabschnittes (C3) nach rechts bzw. links $(\vec{v}=-[\vec{l} \times \vec{\omega}] ; \vec{v}=$ Translationsgeschwindigkeit; $\vec{l}=$ Achsverschiebung; $\vec{\omega}$ = Winkelgeschwindigkeit) und ist bei allen untersuchten Präparaten, sowie bei Prestige $^{\circledast}$ LP, als auch bei Bryan ${ }^{\circledast}$ Cervical Disc zu beobachten. Verursacht durch einen zusätzlichen Versatz der momentanen Drehachse nach lateral, ließ sich für die Präparate HGW 08/06, HGW 10/06 und HGW 11/07 eine individuelle Zunahme der Dorsal- bzw. Ventralverschiebung des oberen Wirbels unter Prestige ${ }^{\circledR}$ LP verzeichnen. Gleiches galt unter Bryan ${ }^{\circledR}$ Cervical Disc für die Präparate HGW 10/06, HGW 11/07, HGW 14/05 und HGW 16/07. Die erhöhte, seitliche Verschiebung des dritten Halswirbels, sowie die damit verbundene Positionsänderung zum angrenzenden Segment, kann den Druck auf die artikulierenden Flächen der Facettengelenke steigern und beeinflussen gleichzeitig die Kinematik des angrenzenden C2/C3-Segmentes (Chang et al. 2007, Zander et al. 2009, Wachowski et al. 2013). Aufgrund dessen ist für den vierten Halswirbel in vivo eine ähnliche Erhöhung der Seitwärtsbewegung denkbar, wodurch wiederum eine Modifikation der C4/C5-Kinematik zu erwarten ist. Aus diesem Grund ist die Entstehung einer Degeneration der angrenzenden Segmente (ALD; „Adjacent Level Disease“) nach totalem Bandscheibenersatz (TDA; „Total Disc Arthroplasty“) 
nicht nur nicht ausgeschlossen, sondern insbesondere bei einer zusätzlichen Dorsal- bzw. Ventralverschiebung mit erhöhter Beanspruchung der Facettengelenke wahrscheinlich. Zum sicheren Beweis dieser Hypothese wären allerdings bisegmentale kinematische Analysen notwendig, die innerhalb der Arbeitsgruppe geplant sind.

Analog zur dorsalen Verlagerung der IHA in Neutralstellung des Segmentes $\left(\alpha=0^{\circ}\right)$ nach TDA, war ein signifikanter Versatz des Schwerpunktes r(s) der gesamten Rastpolkurve nach dorsal zu objektivieren. Eine signifikante Verlagerung in lateraler Richtung (y-Komponente) war nicht zu beobachten. Die Implantation der Prestige ${ }^{\circledR}$ LP führte zu einem signifikanten Versatz des Schwerpunktes der gesamten Rastpolkurve um 4,39 mm nach dorsal, während die Implantation der Bryan ${ }^{\circledR}$ Cervical Disc mit einer Dorsalverlagerung um 6,51 mm einhergeht (siehe 3.6.2.3, Tabelle 3-9). Dadurch lässt sich nachweisen, dass nicht nur die Position der initialen Schraubachse $\left(\alpha=0^{\circ}\right)$ infolge des Bandscheibenersatzes verändert wird, sondern der Schwerpunkt aller momentanen Rotationsachsen eines Bewegungszyklus nach dorsal verschoben ist. Die hohe Variabilität der Verlagerung des Schwerpunktes in Abhängigkeit des TDA hängt damit zusammen, dass hier die Rotationsachsen in großen Auslenkwinkeln ( $\alpha$ bis $-2,4^{\circ}$ und $+2,4^{\circ}$ ) mit in die Berechnung der Koordinaten (x(s); $y(s)$ ) einfließen. Die Betrachtung des Schwerpunktes kleinerer Rotationswinkel ( $\alpha$ zwischen $-0,8^{\circ}$ und $+0,8^{\circ}$ ) wäre daher wahrscheinlich genauer, würde aber wiederum nur einen Teil der Gesamtbewegung einschließen und eine Aussage über die Verhältnisse in hohen Auslenkwinkeln nicht zulassen. Die Verschiebung des Schwerpunktes zeigt, dass die Kinematik für größere Rotationswinkel durch die TDA ebenfalls beeinflusst wird. Zur Art und Weise können die oben angeführten Erklärungen auch für größere Winkel angewendet werden.

\section{Initiale Migrationsrate}

Aufgrund der nahezu gleichbleibenden Ausrichtung der momentanen Rotationsachse im Bereich kleiner Rotationswinkel ( $\alpha$ zwischen $-0,8^{\circ}$ und $+0,8^{\circ}$ ), handelt es sich um eine annähernd ebene Bewegung. Während $v_{x}(0)$, die $x-$ Komponente der initialen Migrationsrate, in allen Messungen ohne Signifikanz blieb, zeigte sich eine signifikante Migration in seitlicher Richtung $\left(\mathrm{v}_{\mathrm{y}}(0) ; \mathrm{y}\right.$-Komponente). Im Rahmen einer Linksrotation wanderte die IHA mit zunehmendem Drehmoment $T_{z}(t)$ und unter dorsaler bzw. zentraler Platzierung der Vorlast nach links. Während der Rechtsrotation war eine entsprechend gleichsinnige IHA-Migration nach rechts zu beobachten. Dieser Zusammenhang ist bemerkenswert, da eine Linksdrehung im Bereich der Lendenwirbelsäule zur Migration der momentanen Rotationsachse nach rechts führt (Nägerl et al. 1992, Nägerl et al. 1995, Mansour et al. 2004, Wachwoski et al. 2009c). In der Hals- und Lendenwirbelsäule findet folglich eine entgegengesetzte IHA- Migration statt. Damit ist die Kinematik dieser beiden Wirbelsäulenabschnitte komplett verschieden. Vermutlich hängt dies mit der Ausrichtung der Drehachse zusammen, welche 
im lumbalen Anteil der Wirbelsäule annähernd parallel zu den Facettengelenken verläuft, während sie im zervikalen Abschnitt nahezu perpendikular zu den Gelenkflächen der Wirbelbogengelenke (C3/C4) steht (Wachowski et al. 2009a, Patwardhan et al. 2010). Die zunehmende Migrationsrate infolge der Prothesenimplantation ist möglicherweise auf den Steifigkeitsverlust und den damit verbundenen Anstieg der Segmentbeweglichkeit nach TDA zurückzuführen. Aufgrund der Teilresektion der Uncovertebralgelenke im Rahmen der Prothesenimplantation, ist anschließend nicht mehr mit einer adäquaten Funktionsfähigkeit zu rechnen (Wachowski et al. 2007, Kang et al. 2010a, Kang et al. 2010b). Diese Teilresektion ist allerdings auf Grund der in der Praxis meist notwendigen Dekompression der nervalen Strukturen unabdingbar (z.B. Snyder und Bernhardt 1989, Snyder et al. 2007, Kotani et al. 2005). Die vermutete Kontrolle einer übermäßigen, seitlichen IHAMigration durch die Gelenke (Wachowski 2005, Wachowski et al. 2007, Wachowski et al. 2009b) wäre somit gestört und könnte nach Implantation der Bryan ${ }^{\circledR}$ Cervical Disk zu einer erhöhten, lateralen IMR ( $\left.\left(<\Delta \mathrm{v}_{\mathrm{ybi}}\right\rangle=2,33 \mathrm{~mm} / \mathrm{deg}\right)$ führen. Dies würde auch die Zunahme der zurückgelegten Migrationsstrecke, besonders unter dorsaler Vorlastposition, erklären, die vor allem nach der Implantation der Bryan ${ }^{\circledR}$ Cervical Disk zu beobachten ist. Durch die nichtsignifikante IMR-Differenz der Prestige ${ }^{\circledast} \mathrm{LP}\left(\left\langle\Delta \mathrm{v}_{\mathrm{ypi}}\right\rangle=0,89 \mathrm{~mm} / \mathrm{deg}\right)$ kann hingegen vermutet werden, dass die Kontrollfunktion der Uncovertebralgelenke zu einem gewissen Grad durch die Prothese übernommen wird. Vorstellbar wäre, dass im Rahmen der Scherbewegung des oberen Wirbels ein seitlicher Kontakt der metallischen Gelenkkugel mit der Gelenkpfanne entsteht. Dies könnte wiederum eine weitere Auslenkung hemmen und damit die laterale IHA-Migration limitieren.

\section{Steifigkeit und Bewegungsausmaß}

Die Steifigkeit eines Bewegungssegmentes und das damit einhergehenden Bewegungsausmaß (ROM) stellen, trotz der Tatsache dass es sich nicht um kinematische Größen handelt, wichtige Parameter bei der Analyse der biomechanischen Eigenschaften dar. Sie zeigen eine deutliche Abhängigkeit von der Position und dem Betrag der axial applizierten Vorlast, woraus sich schlussfolgern lässt, dass in vivo, die Eigenschaften des Systems durch unterschiedliche Muskelaktivierungen beeinflusst werden können. Primär ist in allen Messungen ein Anstieg der Segmentsteifigkeit mit zunehmendem Rotationswinkel $\alpha$ zu erkennen. Für Bewegungen, bei denen die Rotationsachse senkrecht durch das Widerstandszentrum verläuft und keine Gelenkführung auftritt, liegt einer der Gründe dafür in der speziellen Faseranordnung der Anulus fibrosus. Im Rahmen der Rotation werden die scherengitterartig angeordneten Fasern unter Zug gesetzt und hemmen eine überschießende Bewegung. Gleichzeitig kommt es durch eine seitliche Kompression des Nucleus pulposus zu einer Höhenzunahme der Zwischenwirbelscheibe (Kapandji 1985), die experimentell als Schraubsteigung entlang der Drehachse bestimmt werden kann. Dieser so entstehende vertikale Zug erhöht die 
Faserspannung zusätzlich und generiert ein rücktreibendes Drehmoment, welches ebenfalls die Steifigkeit erhöht (Nägerl et el. 1995, Wachowski 2005). Verläuft die momentane Rotationsachse hingegen nicht durch das Widerstandzentrum, kommt es zu einem zusätzlichen Anstieg der Steifigkeit durch die Entstehung von Scherkräften innerhalb der Bandscheibe (Nägerl 1990). Treten während einer Bewegung Gelenkflächen in Kontakt, reduzieren sie einerseits die Freiheitsgrade des Bewegungssystems und senken die Beweglichkeit des Segmentes (siehe 1.5.5 Biomechanische Eigenschaften intakter Segmente, Nägerl 1990, Nägerl et al. 1995), andererseits kommt es infolge der nun auftretenden Achsneigung zu weiteren Wechselwirkungen mit der Bandscheibe. Bedingt durch die Rotation um eine geneigte Achse entsteht eine Verkippung der beiden Wirbel zu einander im Sinne einer Kombinationsbewegung aus Axialrotation und Lateralflexion. Daraus resultiert eine Kompression bzw. Dehnung des Bandscheibenmaterials, wodurch neben dem Schermodul auch der höhere Elastizitätsmodul (siehe 1.2.1.2 Zwischenwirbelscheibe) zum Tragen kommt und die Steifigkeit des Systems weiter zunimmt (Nägerl 1990).

Die Erhöhung der applizierten Vorlast im Widerstandszentrum geht ebenfalls mit einer Zunahme der Steifigkeit einher. Eine Verdreifachung der zentralen Belastung von $20 \mathrm{~N}$ auf $60 \mathrm{~N}$ zeigt in den durchgeführten Versuchen einen durchschnittlichen Anstieg der Steifigkeit um 43\%. Analog sinkt der $\mathrm{ROM}$ von $\approx 4,3^{\circ}$ auf $\approx 3,85^{\circ}$ um annähernd $12 \%$. Dies kann unter anderem durch eine stärkere Kompression bzw. Dehnung der Bandscheibe und eine resultierende Zunahme der inneren Reibung in der Bandscheibe und den Gelenken erklärt werden. Gleichzeitig lässt sich infolge der erhöhten Belastung die frühzeitige Kraftschlüssigkeit aller vier Gelenke postulieren, wodurch die Beweglichkeit auf zwei Freiheitsgrade begrenzt wird, was die Steifigkeit des Segmentes erhöhen würde. Jedoch kommt es bei einer Rotation oder Lateralflexion zu einer raschen Entkopplung von Gelenken, was wiederum die Zahl der Freiheitsgrade erhöht und durch die einhergehende Steifigkeitsreduktion eine weitere Bewegung gewährleistet. Flexion und Extension sind hingegen auch dann möglich, wenn alle vier Gelenke kraftschlüssig sind.

Eine Platzierung der axialen Vorlast ventral des Widerstandszentrums geht mit einem Abfall der Steifigkeit einher. Bringt man beispielsweise $20 \mathrm{~N} 20 \mathrm{~mm}$ vor dem WZ an, zeigt sich eine fast 30\%ige Abnahme der initialen Steifigkeit im Vergleich zur zentralen Belastung. Dies spiegelt sich entsprechend in einem zunehmenden Bewegungsausmaß wieder, welches durchschnittlich um 12\% ansteigt. Eine mögliche Erklärung liegt darin, dass die Wirbelbogengelenke in Prä-Flexion entkoppeln und somit die Gelenkführung verzögert einsetzt bzw. ausbleibt. Dadurch wird die Zahl der möglichen Freiheitsgrade erhöht und die Beweglichkeit des Segmentes nimmt zu. Weiterhin werden durch eine Flexion andere Kontaktpunkte in den Wirbelbogengelenken angesteuert, was, wie in der Lendenwirbelsäule (Nägerl et al. 2009), zu einer geringeren Steifigkeit führen kann. Neben diesen 
kinematischen Gründen, ist die mögliche Abnahme des Drucks und damit der Reibung in den Wirbelbogengelenken nicht zu vernachlässigen (Chang et al. 2007).

Im Unterschied dazu hat eine $20 \mathrm{~mm}$ dorsal des Widerstandszentrums angebrachte Last von $20 \mathrm{~N}$ eine Zunahme der Segmentsteifigkeit um ca. 50\% zur Folge. Dabei sinkt der ROM um 17\%, verglichen mit dem identischen Vorlastbetrag im WZ. Bei dorsaler Vorlastapplikation entsteht eine PräExtension des dritten Halswirbels, sodass von einem frühzeitigen Kraftschluss der Facettengelenksflächen auszugehen ist. Die damit einhergehende Reduktion der möglichen Freiheitsgrade führt zu einem deutlichen Anstieg der initialen Steifigkeit. Zusätzlich wird diese durch die Gelenkführung und die dadurch verursachte Wanderung der IHA aus zentralen Positionen in Richtung des Gelenkes zu größeren Scherkräften innerhalb der Bandscheibe vergrößert (Nägerl 1990). Dieser Mechanismus ist bereits für die Lendenwirbelsäule beschrieben worden (Nägerl et al. 2009).

Die Implantation der Bandscheibenprothesen geht mit einem erheblichen Abfall der Steifigkeit einher. Sie sinkt bei neutraler Belastung ( $20 \mathrm{~N}$ im WZ) um annähernd 36\% beim Bandscheibenersatz durch Prestige ${ }^{\circledR}$ LP und um ca. $45 \%$ bei implantierter Bryan ${ }^{\circledR}$ Cervical Disc. Dieses Phänomen ist neben dem Verlust der oben geschilderten und durch die Bandscheibe bedingten Versteifung unter anderem auf die Resektion von bewegungshemmenden Strukturen im Verlauf der chirurgischen Intervention zurückzuführen. Einerseits werden die Longitudinalbänder durchtrennt, andererseits neben der kompletten Bandscheibe auch Teile der Uncovertebralgelenke entfernt. Demnach ist davon auszugehen, dass nicht nur die Resektion von Bändern und Bandscheibe, sondern auch ein partieller Funktionsverlust der Uncovertebralgelenke zur Reduktion der Segmentsteifigkeit beiträgt. Dies korreliert mit den Ergebnissen von Snyder et al. 2007. Resultierend zeigt sich ein deutlicher Anstieg des Bewegungsausmaßes um rund $1,4^{\circ}$ (32\%) bei Prestige ${ }^{\circledR}$ LP und 1,8 $8^{\circ}$ (42\%) bei Bryan ${ }^{\circledast}$ Cervical Disc (zentrale Vorlast 20 N). Die Abhängigkeit der Steifigkeit bzw. des ROM von Betrag und Position der axialen Vorlast bleibt nach TDA weiterhin erhalten. Dabei ist zu beobachten, dass Veränderungen durch die Vorlastverlagerung zum Teil gleichbleibende Tendenzen aufweisen. So ist beispielsweise die prozentuale Änderung der initialen Steifigkeit und des Bewegungsausmaßes unter ventraler Belastung nahezu identisch mit den Werten der intakten Segmente. Hingegen zeigen Rotationsbewegungen bei dorsal platzierter Vorlast (besonders Bryan ${ }^{\circledR}$ Cervical Disc) einen potentiell etwas größeren Anstieg der Segmentsteifigkeit als vor der Prothesenimplantation (Abb. 4-2). Ein Vergleich der beiden Prothesen untereinander lässt hinsichtlich der Segmentsteifigkeit und des Bewegungsausmaßes nur einen geringen Unterschied erkennen (siehe auch Tab. 3-10 und Tab. 3-11). 


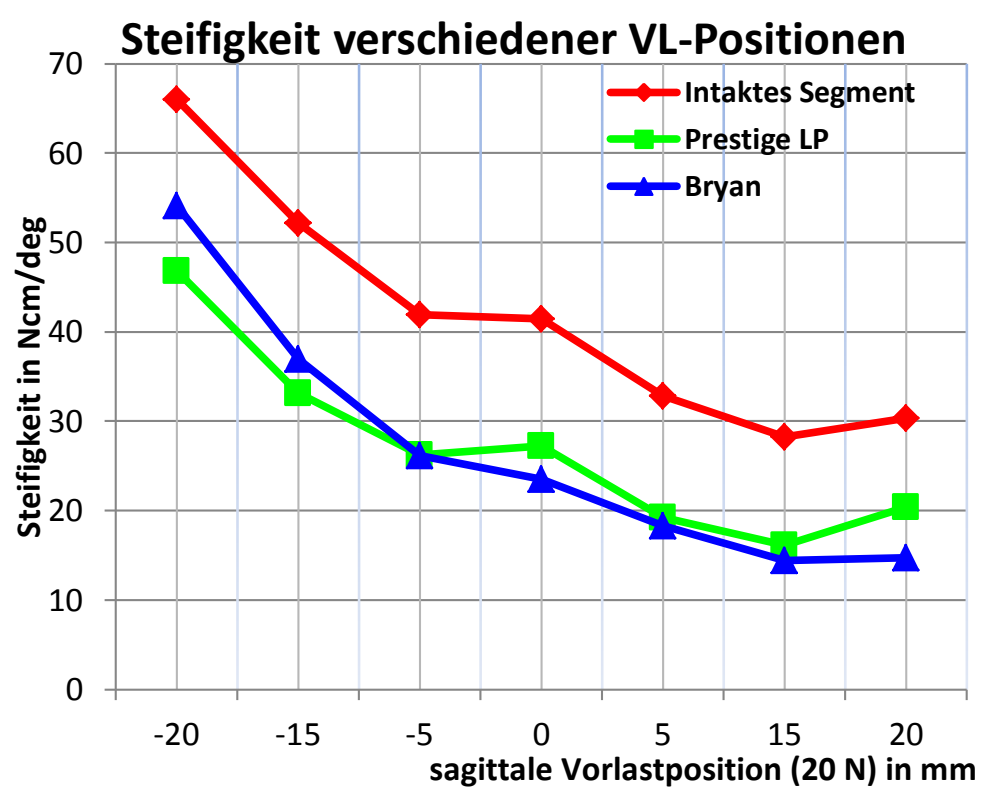

Abb. 4-2: Übersicht über die Entwicklung der initialen Steifigkeit in Abhängigkeit von der sagittalen Vorlastposition. Axialrotation. Primär ist der allgemeine Steifigkeitsverlust nach TDA erkennbar. Bei ventralen Vorlasten (positiv) zeigt sich ein annähernd gleichbleibender Abfall der Steifigkeit, während dorsal gelegene Lasten v.a. mit der Bryan $^{\circledR} \quad \mathrm{zu}$ einem größeren Anstieg der Steifigkeit führen.

Ein Grund für diese Steifigkeitsentwicklung liegt in der schnelleren Dorsalverlagerung der momentanen Schraubachse unter Rotation nach Implantation der Prothesen. Durch diesen Versatz kommt es während der Rotation zur Entstehung größerer Scherkräfte, deren Gegenkräfte eine Zunahme der Steifigkeit bewirken und die Gesamtbeweglichkeit reduzieren. Dieses Phänomen ist bereits für die Lendenwirbelsäule beschrieben worden (Nägerl et al. 2009, Wachowski et al. 2010). Andererseits ist aufgrund der erniedrigten Grundsteifigkeit zu postulieren, dass eine Verschiebung der Vorlast zu einer stärkeren Verkippung des dritten Halswirbels führt. Eine gesteigerte PräExtension könnte den Druck auf die Facettengelenke erhöhen und einen Anstieg der Gelenkreibung verursachen. Zudem ist ein frühzeitiger Eintritt von Kontaktpunkten zwischen den Gelenkflächen denkbar, der wiederum in einem Verlust an Freiheitsgraden resultiert und die Beweglichkeit des Systems reduziert. Die Position der momentanen Schraubachse ist nach TDA deutlich von der Vorlastplatzierung abhängig. Dabei zeigt sich, dass die Differenz der Drehachsenposition zwischen ventraler und zentraler, als auch zwischen zentraler und dorsaler Belastung um ein Vielfaches zunimmt. Durch die erhöhte Ventralverlagerung der IHA bei ventraler Belastung könnte der allgemeine Dorsalversatz der momentanen Schraubachse teilweise kompensiert werden und eine, dem physiologischen Zustand ähnliche, Konstellation der Gelenkartikulation (Entkopplung der Facettengelenke) entstehen. Dies würde die gleichbleibende prozentuale Änderung der initialen Steifigkeit und des Bewegungsausmaßes erklären. 


\subsubsection{Lateralflexion}

Ähnlich der axialen Rotation liegt die momentane Rotationsachse bei der Lateralflexion nicht parallel zum angelegten Drehmoment $T_{x}$, sondern verläuft von dorso-kranial nach ventro-kaudal annähernd parallel zur Sagittalebene. Dabei ist sie um ca. $22^{\circ}$ zum Drehmoment verkippt. Es ist daher erneut eine Kombinationsbewegung aus beiden Bewegungen (Lateralflexion und axiale Rotation) zu beobachten. Die IHA ist im Wesentlichen auf Höhe des dritten Wirbelkörper lokalisiert und wandert im Rahmen einer Linksneigung in Richtung des linken, bei einer Rechtsneigung in Richtung des rechten Facettengelenks, was wiederum auf wechselnde Gelenkführung schließen lässt, die im Verlauf der Bewegung einsetzt. Die scharfen Wendungen, die sowohl bei axialer Rotation, als auch bei Lateralflexion am Ende eines Zyklus zu sehen sind, lassen einen Führungswechsel der Gelenke vermuten, der zum abrupten Richtungswechsel der IHA-Migration führt (Wachowski et al. 2007). Gleichzeitig kommt es zu einer zunehmenden Neigung der Rotationsachse in x-Richtung. Sie liegen dadurch nicht mehr parallel zur Sagittalachse, sondern schwenkt in lateraler Richtung, so dass eine fächerförmige Regelfläche entsteht. Die Implantation der Bandscheibenprothesen ändert an der Ausrichtung und am Wanderungsverhalten der momentanen Schraubachse wenig. Es zeigt sich jedoch eine deutlich weniger scharfe Richtungsänderung am Ende der Bewegung. Stattdessen ist eine bogenförmige Wanderung der IHA zu beobachten. Dies kann als Hinweis darauf verstanden werden, dass ein an dieser Stelle lokalisierter Führungswechsel zwischen den Gelenken nicht mehr stattfindet. In Korrelation zu Voruntersuchungen (Wachowski et al. 2007) kann hier das Fehlen eines Uncovertebralkontaktes postuliert werden. Darüber hinaus verlagert sich die Position der Drehachse prothesenabhängig nach kranial (Presige ${ }^{\circledR}$ LP ca. 5 mm; Bryan ${ }^{\circledast}$ Cervical Disc ca. $8 \mathrm{~mm}$ ). Infolge einer theoretisch möglichen Entkopplung der Wirbelbogengelenke unter ventraler Vorlast könnte sich die horizontale Wanderung der Rotationsachse sowohl beim intakten Segment, als auch nach Bandscheibenersatz verringern. Analog zeigt sich eine Zunahme der seitlichen Migration bei dorsaler Belastung durch die vermutlich frühzeitig einsetzende Gelenkführung. Generell geht die Implantation der Bandscheibenprothesen erneut mit einem erheblichen Verlust an Steifigkeit einher (Presige ${ }^{\circledR}$ LP $-38 \%$; Bryan ${ }^{\circledR}$ Cervical Disc $-50 \%$ ), begleitend steigt das Bewegungsausmaß (Prestige ${ }^{\circledR}$ LP $+27 \%$; Bryan $^{\circledast}$ Cervical Disc $\left.+43 \%\right)$. Die Position der axialen Vorlast nimmt dabei hauptsächlich einen Einfluss auf das Ausmaß der Segmentsteifigkeit. Dorsal gelegen führt Sie, wie bei der Axialrotation zu einem Anstieg, ventral gelegen zu einem Abfall der initialen Steifigkeit, was wiederum durch den frühzeitigen Kraftschluss (dorsale Vorlast) bzw. die Entkopplung (ventrale Vorlast) der Facettengelenke zu erklären ist. Auffällig ist jedoch eine Zunahme der Steifigkeit bei ventraler Vorlast unter Presige ${ }^{\circledR}$ LP. Eine mögliche Erklärung dafür wäre die Entstehung einer erhöhten Reibung innerhalb des Gelenkanteiles der Prothese. Treten die metallische Kugel (oberer Gelenkanteil) und die Pfanne (unterer Anteil) in Kontakt, kommt es im Rahmen der Lateralflexion zu einer 
Gleitbewegung des oberen Gelenkanteils innerhalb der Gelenkpfanne und zur Zunahme der Reibung. Gleichzeitig bleibt der ROM nahezu gleich. Es lässt sich daher vermuten, dass weder die Bandscheibe bzw. die Prothesen, noch die durchtrennten Teile des Bandapparates einen entscheidenden, bewegungslimitierende Einfluss auf die maximale Lateralflexion nehmen. Vielmehr scheint dieser von den artikulierenden Gelenkflächen der Facettengelenke auszugehen, da diese, im Gegensatz zu den Uncovertebralgelenken, bei der Prothesenimplantation unberührt bleiben und ihre Funktion behalten. Der deutliche Abfall der Beweglichkeit bei dorsaler Vorlastposition scheint nach TDA erneut auf die früh einsetzende Gelenkführung zurückzugehen, die im Gegensatz zum intakten Segment durch eine stärkere Prä-Extension infolge der fehlenden ventralen Strukturen, die in Extension angespannt würden, entsteht.

Wie bereits erwähnt, zeigt sich aufgrund der besonderen Ausrichtung der momentanen Schraubachsen eine Kombinationsbewegung aus axialer Rotation und Lateralflexion. Durch variierende Kontaktpunkte der Facettengelenksflächen, die infolge unterschiedlicher, aufeinanderfolgender Muskelaktivierungen entstehen könnten, kann es zu einem fließenden Übergang zwischen den beiden Bewegungsrichtungen kommen. Die Messungen ergeben, dass die Drehachsen $\mathrm{IHA}_{\mathrm{ax}}$ und $\mathrm{IHA} \mathrm{A}_{\text {lat }}$ in kleinen Rotationsintervallen ( $\alpha$ zwischen $-1^{\circ}$ und $+1^{\circ}$ ) parallel zur z-xEbene des experimentell gefundenen Koordinatensystems verlaufen (Abb. 4-3). Angesichts der jeweiligen Neigung ( $1 \mathrm{HA} \mathrm{Ax}_{\mathrm{ax}} \approx 67^{\circ}, \mathrm{IHA} \mathrm{Aat}_{\mathrm{lat}} \approx 22^{\circ}$ zur Horizontalebene) ist davon auszugehen, dass sich die Achsen des intakten Segmentes im Punkt $P_{\text {in }}$ schneiden. Nach der Prothesenimplantation zeigt sich am Beispiel der Bryan ${ }^{\circledR}$ eine Verschiebung der $\mathrm{IHA}_{\mathrm{ax}}$ um $\mathrm{d}_{\mathrm{x}}$ nach dorsal und der $\mid H \mathrm{~A}_{\text {lat }}$ um $\mathrm{d}_{z}$ nach kranial. Die Achsen schneiden sich nun im Punkt $P_{b r}$. Kommt es durch die muskulären Steuerung nun dazu, dass die aus den Winkelgeschwindigkeiten $\omega_{\mathrm{ax}}$ und $\omega_{\text {lat }}$ vektoriell resultierende Winkelgeschwindigkeit $\omega$ auf der definierten Linie $\mathrm{P}_{\mathrm{in}} \mathrm{P}_{\mathrm{br}}$ liegt, wäre die Rotation um die entstehende IHA beim intakten Segment und nach Bryan ${ }^{\circledR}$-TDA dieselbe. Aufgrund der Protheseneigenschaften scheint also die Möglichkeit zu bestehen, manche physiologischen Bewegungen des intakten Segmentes exakt zu reproduzieren. 


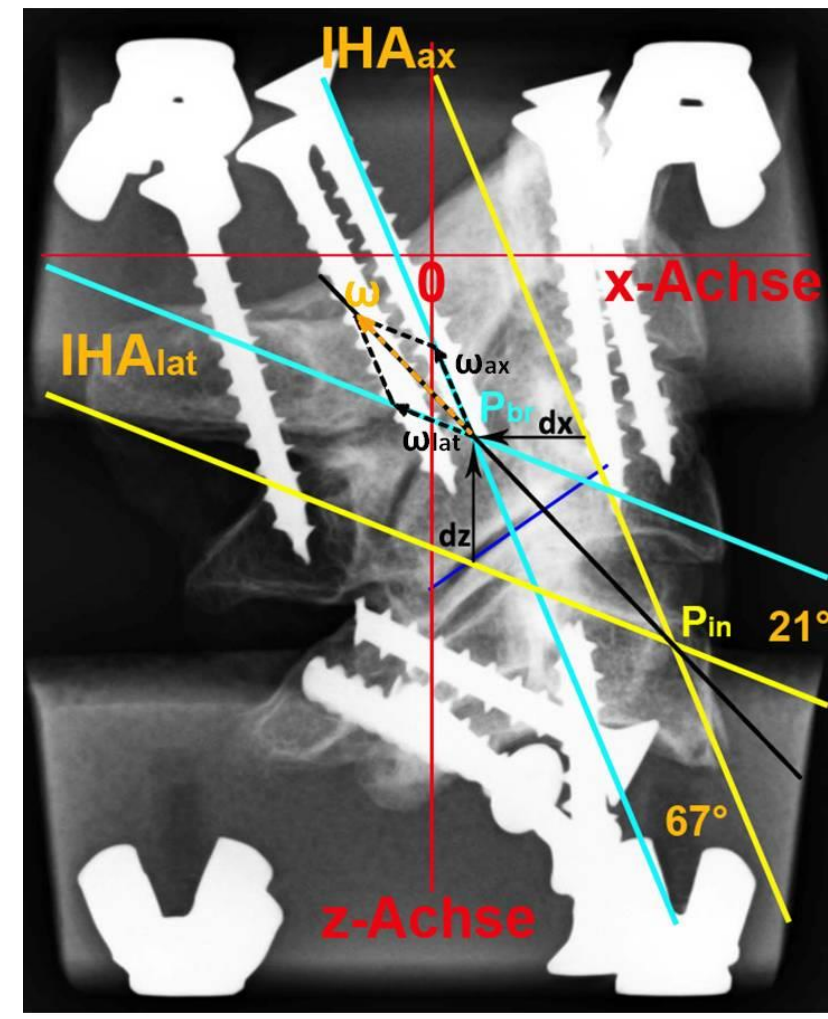

Abb. 4-3: vektoriell resultierende Rotationsachse; Die Drehachsen liegen bei axialer Rotation (IHA ${ }_{a x}$ ) und Lateralflexion ( $\mathrm{IHA}_{\mathrm{lat}}$ ) parallel zur $\mathrm{z}$-X-Achse. Beide Achsen des intakten Segmentes (gelb) schneiden sich im Punkt $P_{\text {in }}$. Nach Bryan ${ }^{\circledR}-T D A$ (türkis) verschieben sich die Achsen um $d_{x}$ bzw. $d_{z}$ und schneiden sich im Punkt $\mathrm{P}_{\mathrm{br}}$. Infolge neu entstehender Kontaktpunkte zwischen den Facettengelenksflächen kann eine resultierende Achse (schwarz) durch beide Punkte eingestellt werden. Die entstehende Rotationsbewegung wäre beim intakten Segment und nach TDA identisch.

$\omega_{\mathrm{ax}}$ : Winkelgeschwindigkeit der IHA bei Axialrotation

$\omega_{\text {lat }}:$ Winkelgeschwindigkeit der IHA bei Lateralflexion

$\omega$ (orange): Winkelgeschwindigkeit der vektoriell resultierenden IHA

\subsubsection{Ventralflexion-Extension}

Im Gegensatz zu den anderen beiden Bewegungsrichtungen verlaufen die momentanen Schraubachsen des intakten Segmentes bei Flexion-Extension annähernd parallel zum angelegten Drehmoment $T_{y}$ und liegen nahezu ortsfest mittig bis dorsal im Bereich des vierten Halswirbelkörpers. Besonders unter dorsaler Vorlastposition ist mit zunehmender Extensionsbewegung $\left(\alpha<-1^{\circ}\right)$ eine schwache Wanderung in Richtung der Facettengelenke erkennbar, was wiederum eine einsetzende Bewegungsführung durch den Kraftschluss der Gelenkflächen vermuten lässt. Hieraus resultiert analog, wie in früheren Studien für die Lendenwirbelsäule bereits geschildert, eine Zunahme der Steifigkeit (Nägerl et al. 2009). Die Ausrichtung, die Position und das Migrationsverhalten der Rotationsachse werden bis auf die beschriebenen Ausläufer bei dorsaler Belastung kaum durch die Verlagerung der axialen Vorlast beeinflusst. Lediglich bei seitlicher Vorlastposition kommt es zu einer veränderten Achsausrichtung. Bei linksseitiger Belastung liegen die Achsen annähernd parallel zu den artikulierenden Gelenkflächen. Dies könnte darauf hinweisen, dass das linke Facettengelenk kraftschlüssig wird und die Bewegung des Segmentes führt. Bei einer rechts gelegenen Vorlast kommt es zu einem Vorzeichenwechsel des Ausrichtungswinkels. Die IHA verläuft nun nicht mehr von links-dorsal nach rechts-ventral, sondern von links-ventral nach rechtsdorsal annähernd parallel zum führenden, rechten Facettengelenk. Dieser Ausrichtungswechsel lässt sich auch nach Implantation der Bandscheibenprothesen nachweisen. Mit der Implantation der 
Bandscheibenprothesen ist analog zur axialen Rotation eine Verlagerung der IHA nach dorsal und analog zur Lateralflexion ein Versatz nach kranial zu beobachten. Die Ausrichtung der Achsen bleibt dabei nahezu konstant, sodass sie weiterhin annähernd parallel zum Drehmoment $T_{y}$ liegen. Zudem ist ein deutlicher Anstieg der initialen Steifigkeit zu verzeichnen. Dieser Befund scheint zunächst etwas paradox, wenn man bedenkt, dass das applizierte Drehmoment von 2,25 Nm auf 0,85 Nm reduziert werden musste. Grund dafür war einerseits die immense Instabilität der Versuchsobjekte in dieser Bewegungsrichtung auf Grund der fehlenden, die Extension limitierenden, ventralen Bandstrukturen, andererseits, dass apparaturbedingt das erfassbare Bewegungsausmaß für die Ventralflexion und Extension erheblich limitiert war. Auf der anderen Seite ist zu beachten, dass bei Ventralflexion und Extension der Elastizitätsmodul der Bandscheibe bzw. der Prothesen einen entscheidenden Einfluss auf die Segmentsteifigkeit hat. Im intakten Segment ist davon auszugehen, dass es neben einer ventralen bzw. dorsalen Abscherung zu einer vorderen bzw. hinteren Kompression der Bandscheibe kommt und so die Reibung ansteigt. Je nach Elastizität kann dies zu einer erhöhten Steifigkeit beitragen. Mit der Resektion der Bandscheibe und dem Ersatz durch eine Prothese könnte ein Großteil der Elastizität dadurch verloren gehen, dass durch einen Kraftschluss des neu entstehenden, künstlichen Gelenkes die Zahl der möglichen Freiheitsgrade sinkt. Dies ist besonders bei der Neigung in ventraler Richtung zu erwarten. Aufgrund der nach kranial verlagerten IHA verkürzt sich zudem der Abstand des beweglichen Segmentanteiles zum Rotationszentrum. Demzufolge sinkt die ventrale Abscherung des oberen Wirbels, wohingegen die kompressive Komponente auf das Bandscheibenfach erhöht wird. Die Bryan ${ }^{\circledR}$ Cervical Disc (Kunststoffkern), als auch die rein metallische Prestige ${ }^{\circledR}$ LP sind allerdings kaum komprimierbar. Eine Ventralneigung um die dorso-kaudal der Prothesen liegende IHA ist daher mit einer Erhöhung der Reibung nach Kraftschluss verbunden. Durch die Kompression des ventralen Segmentanteils kann postuliert werden, dass die Zugspannung der Ligamenta interspinalia zunimmt die Kapselspannung der Facettengelenke ansteigt und die Bewegung limitiert wird. Eine Neigung nach dorsal hat eine gleichsinnige Verlagerung der IHA zur Folge und wird primär durch die einsetzende Gelenkführung der Wirbelbogengelenke begrenzt. Es lässt sich vermuten, dass es besonders unter dorsal platzierten Vorlasten mit zunehmendem Rotationswinkel $\alpha$ zu einem vermehrten Druck auf die Gelenkflächen kommt. Die Zunahme der Steifigkeit könnte zudem zusätzlich in einem möglichen Anstieg der Reibung, aufgrund veränderter Druckverhältnisse, begründet sein. 


\subsubsection{Biomechanische Schlussfolgerung}

Die Implantation der untersuchen Bandscheibenprothesen führt zu einer deutlichen Veränderung der kinematischen Eigenschaften des Index-Segmentes. Neben einer vorlastabhängigen Abnahme der Segmentsteifigkeit, die mit einem entsprechenden Anstieg des Bewegungsausmaßes einhergeht, zeigt sich eine veränderte IHA-Migration im Rahmen einer Rotation. Analog zum intakten Bewegungssegment bleibt die Wanderung ebenfalls abhängig von der Position und dem Betrag der axialen Vorlast. Darüber hinaus kommt es nach der Implantation der Prestige ${ }^{\circledR}$ LP Cervical Disc bzw. Bryan ${ }^{\circledR}$ Cervical Disc zu einem signifikanten Versatz der momentanen Schraubachse. Im Verlauf eines Bewegungszyklus resultiert aus dieser erheblichen Parallelverschiebung der Rotationsachse wahrscheinlich eine zusätzliche Translation des beweglichen Segmentabschnittes (C3). Die damit einhergehende Positionsänderung zum angrenzenden Segment könnte den Druck auf die artikulierenden Flächen der Facettengelenke steigern und die Kinematik des benachbarten C2/C3Segmentes beeinflussen (Chang et al. 2007, Zander et al. 2009, Wachowski et al. 2013). Eine derartige Zunahme der Translation ist für den vierten Halswirbel in vivo ebenfalls denkbar, wodurch wiederum eine Modifikation der C4/C5-Kinematik diskutiert werden könnte. Die Dorsal- bzw. Ventralverschiebung und die daraus entstehende erhöhte Beanspruchung der Facettengelenke, könnte eine Degeneration der angrenzenden Segmente (ALD; „Adjacent Level Disease“) nach totalem Bandscheibenersatz (TDA; „Total Disc Arthroplasty“) begünstigen. Die Entstehung der ALD ist somit mit vollständig auszuschließen. 


\subsection{Methodendisskussion}

\subsubsection{Kraftsystem}

Physiologisch unterliegt die Halswirbelsäule der Gewichtskraft des Kopfes und den angreifenden Zugkräften verschiedener Muskelgruppen (siehe Kapitel 1.2.1.5). Diese einwirkenden Kräfte lassen sich auf verschiedene Kraftschrauben reduzieren, deren Konzept als die geometrische Struktur aller simultan wirkenden Kräfte auf ein System definiert ist. Die Reduktion der unendlich großen Anzahl an möglichen Kräftekonstellationen auf eine Kraftschraube hat den Vorteil, dass diese im vorliegenden Versuchsaufbau beeinflussbar, reproduzierbar und konstant ist. Mit ihren sechs Parametern ist die Kraftschraube leicht zu kontrollieren und hat, aufgrund des in dieser Studie verwendeten Versuchsansatzes, keinerlei Einfluss auf die Führung der Bewegung. Es ist zu bedenken, dass die Simulation komplexer Kraftverläufe, wie sie in vivo durch das Zusammenspiel einzelner Muskeln oder Muskelgruppen entstehen, äußerst kompliziert ist und zudem einen Einfluss auf alle Parameter der vorliegenden Kraftschraube nehmen würde. Die Analyse der Versuchsdaten wäre dadurch schwer kontrollierbar und nahezu nicht reproduzierbar, weshalb Versuchsansätze mit stärkerer Annäherung an die physiologische Muskelanordnung und deren Kräfte kaum realisierbar ist. Gleichzeitig muss berücksichtigt werden, dass bereits kleine Änderungen der einwirkenden Muskelkräfte zu einer veränderten Druckbelastung in den Facettengelenken führen kann (El-Bohy et al. 1989). Dies könnte wiederum mit variierenden Kontaktpunkten der Gelenkflächen und veränderten Führungsbedingungen einhergehen und das Verhalten des Systems beeinflussen. Außerdem konnten Parnianpour et al. bereits 1989 nachweisen, dass die Kontraktionskraft der Muskulatur inkonstant ist und dauerhaft höchstens 15\% der maximal möglichen Muskelkraft beträgt (Parnianpour et al. 1989). Physikalisch gesehen ist es daher für den gewählten Versuchsansatz gerechtfertigt, die verschiedenen Kraftkonstellationen auf eine Kraftschraube zu reduzieren, welche ohne Qualitätsverlust agiert. Um der Forderung nachzukommen, die verschiedenen Parameter der Kraftschraube kontrolliert beeinflussen zu können, ist sie gar als unabdingbar zu betrachten.

\subsubsection{Kompressive Kraftkomponente}

Der Einfluss von axialen Vorlasten auf die Bewegungsstruktur eines Wirbelsäulensegmentes konnte bereits in zahlreichen Studien nachgewiesen werden (Panjabi et al 1976, Edwards et al. 1987, Ahmed et al. 1990, Schildhauer et al. 1994, Kalscheuer 2001, Wachowski et al. 2007, Wachowski et al. 2009b, Wachowski et al. 2010). In dieser Studie wurde eine frei hängende, rückwirkungsfreie Vorlast verwendet, da der Hauptfokus auf der axialen Rotation lag und jegliche Führung der Vorlast zu Artefakten und fehlerhaften Ergebnissen führen würde (Patwardhan et al. 2010, Cripton et al. 2000). Die Applikation der Vorlast durch einen Stempel, wie es beispielsweise von Rousseau et al. 
gehandhabt wurde (2006), führt unweigerlich zu einer Kompression oder Scherbewegungen und nimmt Einfluss auf die Führung des Segmentes während der Bewegung. Allerdings muss berücksichtigt werden, dass jede Art der Vorlastapplikation mit Artefakten verbunden ist (Cripton et al. 2000). Daher verwenden einige Arbeitsgruppen Vorlasten lediglich bei Flexion bzw. Extension (Patwardhan et al. 2010). Da unter physiologischen Bedingungen jedoch eine muskuläre Vorspannung/Vorlast auch in den anderen Bewegungsrichtungen gegeben ist, kann die Aussagekraft der Daten für physiologischen Verhältnisse hinterfragt werden. Das Anlegen einer frei hängenden, nicht geführten Vorlast hat den Vorteil, dass keine artifiziellen Scherkräfte induziert werden und scheint daher die besten Methode für die axiale Rotation darzustellen (Cripton et al. 2000). Der Nachteil einer nicht geführten Vorlast ist die Entstehung eines zusätzlichen Drehmomentes, was zur Verkippung des oberen Wirbels führt. Auf diese Weise kann es zu Belastungsveränderungen im Gelenksystem kommen und folglich neue Kontaktpunkte entstehen (Nägerl et al. 1995). Da jedoch kein signifikanter Unterschied zum unbelasteten Segment zu beobachten war, scheint dieses Phänomen bei der in dieser Studie verwendeten Art der axialen Vorlast auszubleiben, da die Ergebnisse eine Abnahme des ROM bei dezentraler Vorlastposition wiederspiegeln. Die frei hängende Vorlast scheint daher auch für die beiden anderen Bewegungsrichtungen die richtige Vorgehensweise gewesen zu sein. Die spezielle Aufhängung der Führungsschiene am Stahlkreuz, wie sie in dieser Studie Anwendung fand (Abb. 2-11), hatte den Vorteil, dass sie frei beweglich war und somit der Bewegung des Segments folgen konnte. Mit einer maximalen Belastung von $50 \mathrm{~N}$ lag die applizierte Vorlast ungefähr im Bereich der Gewichtskraft des menschlichen Kopfes. Größere Vorlastbeträge wurden aufgrund des steigenden Risikos für irreversible Verletzungen, bei Nichtvorhandensein aktiver, muskulärer Stabilisierung und Sicherung, nicht verwendet.

\subsubsection{Drehmoment}

Der in dieser Studie verwendete Betrag des Drehmomentes $(2,25 \mathrm{Nm})$ wurde experimentell ermittelt, indem es schrittweise so lange erhöht wurde, wie die Drehmoment-Drehwinkel-Kurve eine physiologische Gestalt zeigte und das Segment ein physiologisches Bewegungsausmaß aufwies. Dadurch konnte gewährleistet werden, dass eine Überlastung durch zu hohe Krafteinwirkung ausblieb, die in einer Schädigung des Materials resultieren würde. Dies führte zu einem vergleichbaren Wert des empirisch festgelegten Betrages von 2,5 Nm, der in den meisten Studien verwendet wird (Cheng und Welch 2011). In Kapitel 2.3.2 „Applikation von Kräften“ wird dargelegt, dass der Aufbau des Messsystems die Applikation eines reinen, linear ansteigenden Drehmomentes ermöglicht und keinerlei Einfluss auf die Führung der Segmentbewegung besteht.

Aufgrund der starken Beweglichkeit bei Ventralflexion und Extensionsbewegungen und des eingeschränkten Aufzeichnungsvermögens in dieser Bewegungsrichtung war eine Reduktion des 
angelegten Drehmomentes nach TDA erforderlich. Um die Abweichung vom ursprünglich verwendeten Betrag von 2,25 Nm so gering wie möglich zu halten, erfolgte eine sukzessive Senkung des Drehmoments $T_{y}$, bis eine vollständige Aufzeichnung wieder möglich war ( $\left.T_{y}=0,85 \mathrm{Nm}\right)$. Die Art und Weise der Applikation wurde nicht geändert, sodass weiterhin ein rückwirkungsfreies, reines Drehmoment vorlag.

\subsubsection{Messsystem}

Für die Analyse der Bewegungsstruktur der Wirbelsäule ist eine Aufzeichnung des Bewegungsablaufs in differenziell kleinen Winkeln erforderlich, da die Ermittlung des Bewegungsausmaßes und der Steifigkeit, welche in vielen Arbeitsgruppen als zentrale Parameter angesehen werden, bei weitem nicht ausreichend ist (Patwardhan et al. 2010, Lazaro et al. 2010, Kowalczyk et al. 2011). Dies erfordert jedoch eine exakte Einstellung der Messapparatur, da bereits kleine Fehler in den gemessenen Daten zu einer systematischen Verfälschung der Ergebnisse führen würden. Die größte Problematik liegt dabei in der korrekten Positionierung der Lagesensoren im raumfesten Koordinatensystem (Schäfer 1995). Mögliche Fehlerquellen können hier in der Fertigungstoleranz der Messapparatur oder in der fehlerhaften manuellen Tastereinstellung im Halterahmen liegen. Aufgrund der optimierten Anordnung der Lagesensoren für die Vermessung der Axialrotation ergibt sich das bereits erwähnte Problem, dass in den Versuchen zur Ventralflexion-Extension die Aufzeichnung des vollständigen Bewegungsausmaßes nicht möglich ist. Dies gilt besonders bei dorsal gelegener Vorlast und nach Prothesenimplantation. Während die eingelesenen Tasterwerte als sehr valide zu betrachten sind, da sie unmittelbar am Versuchsobjekt aufgenommen werden, enthalten die Berechnungen zur Schraubachse formelbedingt eine nicht zu verachtende Fehlerfortpflanzung. Dennoch kann davon ausgegangen werden, dass die Ergebnisse der acht Halswirbelpräparate nicht durch die Messapparatur beeinflusst werden, da in den Vorversuchen zur Überprüfung der Apparatur mittels Präzisionsschraube und Bandscheibenmodell die Validität des Messsystems nachgewiesen werden konnte, indem die gemessenen Werten den jeweiligen Erwartungen entsprachen.

\subsubsection{Präparate}

Um möglichst physiologische Ergebnisse zu erhalten, ist bei der Wahl der Versuchsobjekte in erster Linie auf deren Unversehrtheit zu achten. Ideal wäre es frische Präparate zu verwenden, die von jungen Erwachsenen abstammen und keinerlei pathologische Veränderungen aufweisen. Die Verfügbarkeit derartiger Messobjekte ist allerdings sehr begrenzt, sodass notgedrungen nach Alternativen gesucht werden muss. Eine Möglichkeit wäre die Verwendung von Tierpräparaten. 
Allerdings sind die Ergebnisse in ihrer Aussagekraft über die Kinematik der menschlichen Wirbelsäule zu bezweifeln, allein schon aufgrund der ersichtlich veränderten Grundvoraussetzungen (aufrechter Gang des Menschen im Gegensatz zum Vierbeinergang). Eine andere denkbare Option wäre der Gebrauch von Kunststoffmodellen. Sicherlich lässt sich das anatomische Gerüst in der heutigen Zeit problemlos nachbilden, eine realistische Simulation der physiologischen Weichteilstrukturen ist jedoch nicht zu erwarten. Ein Rückschluss auf die Verhältnisse und Auswirkung in biologischen Materialien ist daher nicht ohne weiteres möglich. In dieser Studie wurde daher auf fixierte, humane Präparate zurückgegriffen. Da diese hauptsächlich von älteren Personen abstammen, war der Ausschluss pathologischer und altersbedingter Veränderungen von hoher Priorität. In Zusammenarbeit mit einem erfahrenen Radiologen konnten diese mittels modernster Röntgen- und computertomographischer Aufnahmen ausgeschlossen werden. Zusätzlich erfolgte eine Kontrolle nach jedem Implantationsverfahren um eine mögliche iatrogene Schädigung der Präparate erneut auszuschließen und den korrekten Sitz der Prothese im Bandscheibenfach zu überprüfen.

Da die Steifigkeit konservierter Wirbelsäulenpräparate zudem von der Art der Konservierungslösung beeinflusst werden kann (Wilke et al. 1996), wurde eine schonende Fixationslösung aus Wasser, destilliertem Alkohol, Glyzerin, Formalin, Thymol und Salizylsäure (Fanghänel und Schultz 1962, Fanghänel 2009) verwendet. Es muss allerdings berücksichtigt werden, dass eine Änderung der Segmentsteifigkeit nicht gleichbedeutend mit einer Änderung der Bewegungsstruktur ist, da diese primär von der Morphologie der Gelenke beeinflusst wird, statt von den elastischen Strukturen des Bewegungssegmentes (Nägerl et al. 1992, Nägerl et al. 1995, Kalscheuer 2001). Demzufolge ist eine ebensolch schonende Konservierungslösung zu empfehlen, welche weder die Gelenkmorphologie beeinflusst, noch die elastischen Segmentanteile härter werden lässt, als die knöchernen Strukturen und folglich die Führungsdominanz der Gelenkflächen erhalten bleibt. Um einer Austrocknung während der Experimente vorzubeugen, wurden die Präparate vor, während und nach jeder Messreihe mit der konservierenden Tinktur benetzt. Pathologische Veränderungen im Verlauf der Messungen waren nicht zu beobachten. Die Einbettung der Versuchspräparate mittels des kaltpolymerisierenden Kunststoff Weitur ${ }^{\circledR}$ Press (Standard) ermöglichte es, eine indirekte Verbindung zwischen dem Präparat und der Apparatur herzustellen. Dies hatte den Vorteil, dass das Untersuchungsmaterial keinen ansetzenden Strukturen, die zu einer möglichen Schädigung führen konnten, ausgesetzt war. Darüber hinaus wurde so die Grundlage dafür geschaffen, dass das Versuchsobjekt stets die gleiche Ausgangsposition einnahm, wobei von minimalen Abweichungen bei jedem Einbau abgesehen werden muss. Somit waren die Ergebnisse jederzeit reproduzierbar. 


\subsubsection{Implantation der Bandscheibenprothesen}

Der Ersatz der Bandscheibe durch eine künstliche Prothese stellt einen unwiderruflichen Eingriff in den physiologischen Status eines Lebewesens dar. Daher war eine korrekte und präzise Vorgehensweise die Grundvoraussetzung einer solchen Intervention.

Eine Problematik, die einen direkten Vergleich der verfügbaren Studien erschwert, ist die unterschiedliche chirurgische Verfahrensweise bei der Implantation der Bandscheibenprothesen. Einige Arbeitsgruppen resezieren lediglich den Nucleus pulposus und Teile des Anulus fibrosus und verwenden kleine Implantate, während andere die Bandscheibe komplett extrahieren und größere Prothesen einsetzen. Dabei hat der Erhalt bzw. die Durchtrennung des hinteren Längsbandes (Lig. longitudinale posterius) einen wesentlichen Einfluss auf die Steifigkeit und das Bewegungsausmaß (McAfee et al. 2003). Ein direkter Vergleich der gewonnen Erkenntnisse ist daher nur bei identischer chirurgischer Herangehensweise und der Verwendung der gleichen Prothesen bedenkenlos möglich (McAfee et al. 2003, McAfee et al. 2006, Patwardhan et al. 2010). Bei der Analyse der kinematischen Eigenschaften (Lokalisation und Ausrichtung der Rotationsachse, sowie deren Migrationsrate) spielt dieser Aspekt jedoch eine untergeordnete Rolle, da die elastischen Bestandteile lediglich einen marginalen Einfluss auf diese Parameter haben. Dem gegenüber ist der Sitz der Implantate im Intervertebralraum von wesentlich höherer Bedeutung. So konnte nachgewiesen werden, dass besonders Prothesen mit festem Rotationzentrum, je nach ihrer Positionierung, neben der Steifigkeit und dem Bewegungsausmaß, auch die Drehachse beeinflussen können (Patwardhan et al. 2010). Ein weiterer Aspekt ist der Erhalt bzw. die Resektion der Uncovertebralgelenke, deren biomechanische Eigenschaften, sowie die richtige Art und Weise ihrer Behandlung bei klinisch relevanten, degenerativen Veränderungen kontrovers diskutiert werden (Kang et al. 2010a). Einige Arbeitsgruppen konnten anhand unterschiedlicher Resektionsausmaße an den Procc. uncinati und gleichzeitigem TDA einen Effekt auf Steifigkeit und Beweglichkeit nachweisen, wobei die durchgeführte chirurgische Maßnahme auf die Dekompression schmerzverursachender, nervaler Strukturen und die Beschwerdelinderung bei Radikulopathie abzielte (Snyder und Bernhardt 1989, Snyder et al. 2007, Kotani et al. 2005). Andererseits konnten Kang et al. zeigen, dass selbst bei unversehrten Uncovertebralgelenken während einer Bewegung eine signifikante Reduktion des Druckes auf die Gelenkflächen stattfindet (2010), die eine Modifikation der Steifigkeit und des ROM nach sich zieht. Diese unterschiedlichen Erkenntnisse führen letztendlich dazu, dass der kinematische Einfluss der Uncovertebralgelenke nach TDA generell bezweifelt werden kann. In der vorliegenden Studie wurden zunächst eine komplette Entfernung der Bandscheibe, sowie, wie von den meisten Arbeitsgruppen empfohlen, eine Durchtrennung des hinteren Längsbandes vollzogen. Diese Vorgehensweise wird zudem in der klinischen Praxis empfohlen, um eine weites gehende Dekompression der dorsal gelegenen Strukturen zu ermöglichen. Es folgte die Präparation eines zu 
den Grund- und Deckplatten der angrenzenden Wirbel parallelen Intervertebralspaltes und entsprechend der Empfehlung des Herstellers, die anschließende Implantation der Prothesen in sagittaler Ausrichtung und in der Medianlinie des Bandscheibenfaches (Medtronik 2004, Patwardhan et al. 2010). Dabei fand eine Teilresektion der Uncovertebralgelenke statt. Abschließend wurde die Lage der Wirbel zueinander kontrolliert, um eine idiopathische Extensionsstellung auszuschließen.

\subsection{Probemessungen}

\section{Präzisionsschraube}

Um die Genauigkeit und Kalibration der Messapparatur vor den Messungen mit biologischen Versuchsmaterialien zu validieren, wurden zunächst Experimente an einer Präzisionsschraube, deren Eigenschaften bekannt sind, durchgeführt. Die hohe Übereinstimmung der gemessenen Daten mit den Erwartungswerten beweist die Genauigkeit der Messapparatur (siehe Tab. 3-1). Den Abweichungen können verschiedene Ursachen zugrunde liegen: einerseits die Fertigungstoleranz des Herstellers, andererseits Ungenauigkeiten bei der Einbettung und Positionierung der Schraube selbst. Jedoch ist die Differenz zwischen den Werten vernachlässigbar klein.

\section{Bandscheibenmodell}

Anhand der Ergebnisse der Messungen am Bandscheibenmodell lassen sich erste Vermutungen über die tatsächlichen Abläufe, sowie die kinematischen Eigenschaften eines Wirbelsäulenpräparates anstellen. Während beim reinen Bandscheibenimitat die momentanen Drehachsen über den gesamten Bewegungszyklus zentral im Modell lagen, wanderte die IHA nach dem Einbau der Facettengelenkimitate mit zunehmendem Rotationswinkel aus der zentralen Position in Richtung des führenden Gelenkes. Diese Beobachtung stützt die Annahme, dass die Bewegung eines Wirbelsäulensegmentes, sowie die Migration der IHA durch den Kraftschluss eines oder mehrerer Gelenke geführt werden. Ein möglicher Einfluss der Vorlastposition auf die Gelenkführung kann mit den Ergebnissen unter ventraler Vorlastposition postuliert werden. Dabei scheint die Interaktion zwischen den stählernen Gelenkelementen auszubleiben, da die Schraubachse wieder zentral im Bandscheibenmodell lag.

Aufgrund der viskoelastischen Eigenschaften des Bandscheibenmodells kam es im DrehmomentDrehwinkel-Diagramm zur Entstehung einer Hysterese, weshalb hookesches Verhalten des Materials bestätigt werden konnte. Es zeigte sich eine hockey-schlägerartige Form der $\alpha(T)$-Kennlinien, welche bei linear elastischen Eigenschaften und der Applikation eines sinusartig entstehenden Drehmomentes durch Dehnung bzw. Stauchung des Bandscheibenimitates erwartet wird. 


\section{Modellmessung Prestige ${ }^{\circledR}$ LP Cervical Disc}

Anhand der Probemessungen mit der Prestige ${ }^{\circledR}$ LP konnten erste Beobachtungen zum Verhalten unter Anlage einer axialen Vorlast $(60 \mathrm{~N})$ und eines zyklischen Drehmomentes gemacht werden. Aufgrund der enormen Instabilität des Kugelgelenkes war es unumgänglich, die Applikation des Drehmomentes manuell durchzuführen, da die beiden Prothesenteile sonst gegeneinander verkippten. Aus diesem Grund war auch die Anlage der axialen Vorlast lediglich zentral möglich.

Grundsätzlich handelte es sich bei den Versuchen um eine ebene Bewegung. Die Rotationsachsen lagen in den Versuchen ohne Facettengelenkimitate ortsfest, zentral in der Prothese. Eine Schraubsteigung war aufgrund der aus Metall gefertigten Artikulationsteile der Prothese nicht zu beobachten oder zu erwarten. Das Fehlen viskoelastischer Eigenschaften spiegelte sich erwartungsgemäß in der Drehwinkel-Drehmoment-Kurve wieder. Ein hookesches Verhalten, wie es im Bandscheibenmodell zu beobachten war, fehlte vollständig.

Im zweiten Versuchsteil wurde zusätzlich Facettengelenkimitate angebracht. Während der Rotationsbewegung konnte nun mit zunehmendem Rotationwinkel ein Inkrafttreten der Metallflächen der künstlich angelegten Facettengelenke beobachtet werden. Dieser Widerstand führte zu einer Translation des oberen Prothesenteils (Kugel) gegenüber der unteren Einheit (Pfanne). Aufgrund des geringen Spielraumes in lateraler Richtung, traf die Gelenkkugel alsbald an den Pfannenrand und führte zu einer Verkippung der Prothesenteile gegeneinander. Aufgrund der fehlenden Stabilisierung durch Weichteile, kann es dabei zu Sub-/Luxationsereignissen der Prothese gekommen sein. Dies könnte die Wanderung der IHA bei hohen Rotationswinkeln aus der zentralen Position und vor alllem die Verkippung der Drehachse um bis zu $59^{\circ}$ in der Sagittalebene und bis ca. $29^{\circ}$ in der Horizontalebene begründen. Der geradlinige Verlauf in der Drehwinkel-DrehmomentKurve blieb aufgrund des Fehlens viskoelastischer Eigenschaften weiterhin annähernd geradlinig.

\section{Ermittlung des Widerstandszentrums}

Als Widerstandszentrum bezeichnet man den Ort, an dem das Anlegen einer axialen Vorlast keine Verkippung des oberen Wirbels zu Folge hat. Ausgehend von diesem Punkt werden nach einem festgelegten Schema Vorlasten appliziert (siehe 2.3.2 - Applikation von Kräften). Der Grund für die experimentelle Ermittlung des Widerstandszentrums war, dass auf diese Weise sichergestellt werden konnte, dass die Untersuchungsbedingungen (Position der applizierten Vorlast) für die acht Versuchsobjekte, vor und nach Prothesenimplantation, gleich blieben und die Ergebnisse vergleichbar waren. 


\subsection{Ausblick}

Mit den Erkenntnissen aus dieser Studie drängen sich erneut Fragen zu den Auswirkungen der TDA („Total Disc Arthroplasty“) auf die angrenzenden Wirbelsäulenabschnitte eines mit Bandscheibenprothesen versorgten Wirbelsäulensegmentes auf. Besonderes Interesse gilt dabei der Fragestellung nach abnormen Belastungen im Bereich der Gelenkfacette und deren Folgen auf die kinematischen Eigenschaften der Nachbarsegmente. Daher stellt die Erhebung neuer Daten über mehrere Segmenthöhen einen sinnvollen Ansatz zur Gewinnung neuer Erkenntnisse dar. In diesem Zusammenhang müsste jedoch eine neue Messapparatur entwickelt werden, da unsere Arbeitsgruppe nur über ein mechanisches Präzisionsmesssystem für monosegmentale Bewegungen verfügt, das auf bisegmentale Messungen nicht erweitert werden kann. Unser Ziel ist daher die Etablierung einer neuen, optischen Messmethode, mit welcher in In-vitro-Experimenten die räumliche Kinematik zweier angrenzender Segmente gleichzeitig so präzise vermessen wird, dass die mechanische Belastung der Gelenkfacetten berechnet werden kann. Diese Messungen könnten zudem wichtige Anhaltspunkte über das Zusammenspiel der gesamten Wirbelsäule liefern. Gleichzeitig wäre ein Vergleich mit den Erkenntnissen aus den Studien an einzelnen Segmenten möglich, wodurch deren Gültigkeit validiert werden könnte. Ein weiteres Ziel stellt daher eine analoge, hochauflösende Untersuchung anderer Segmenthöhen dar.

Darüber hinaus sollten die Untersuchung einer höheren Anzahl an Präparaten angestrebt werden, damit individuelle Eigenschaften statistisch heraus-gemittelt werden können. Aufgrund der geringen Zahl der bislang untersuchten Versuchsobjekte ist ein Ausschluss von eindeutig pathologisch veränderten Präparaten anzuraten, da diese wahrscheinlich zu einer übermäßigen Ergebnisverfälschung führen würden.

Mit der Prestige ${ }^{\circledast}$ LP Cervical Disc und Bryan ${ }^{\circledast}$ Cervical Disc wurden in dieser Studie zwei verschiedene, zervikale Bandscheibenprothesen untersucht. Die Vielfalt der verfügbaren Prothesen ist deutlich höher, sodass eine weitere Zielsetzung in der Untersuchung anderer Bandscheibenprothesen und anderer Wirbelsäulenabschnitte (z.B. lumbal) liegt.

Perspektivisch wären ebenso Studien über die Auswirkungen der Standard-Operationen (Fusion, Bandscheibenprothese) auf die räumliche Kinematik und interne Belastung eines versorgten Segmentes und gleichzeitig der angrenzenden Etage erstrebenswert. So ließen sich pathologische Bewegungsabläufe und Gelenkbelastungen erfassen und Aussagen über eine biomechanisch begründbare, vorzeitige Degeneration treffen. Die gewonnen Erkenntnisse sollten zudem in langfristig angelegten, klinischen Studien überprüft werden. Dabei müssten die prä- und postoperativen Beschwerden objektiv verglichen und in systematischen Verlaufskontrollen kontrolliert werden. 
Wünschenswert wäre schlussendlich die Entwicklung einer Bandscheibenprothese, die in ihren kinematischen Eigenschaften die physiologischen Abläufe des versorgten Segmentes ganzheitlich imitieren kann und somit ohne Auswirkungen auf die Kinematik der Wirbelsäule bleibt. 


\section{Zusammenfassung}

\subsection{Stand der Wissenschaft}

Degenerative Veränderungen sowie die traumatische Schädigung der Bandscheiben stellen häufige und zudem gesellschaftlich relevante Krankheitsbilder dar. In der operativen Versorgung gilt die Fusion, das heißt die Entfernung der Bandscheibe mit anschließender Versteifung des Bewegungssegmentes, weiterhin als Standardtherapie. Als mögliche Langzeitfolge wird von vielen Autoren die erhöhte Wahrscheinlichkeit der Degeneration der angrenzenden Segmente („Adjacent Segment Disease“; ASD oder „Adjacent Level Disease“; ALD) postuliert. Mit der Implantation von Bandscheibenprothesen („Total Disc Replacement“; TDR oder „Total Disc Arthroplasty“; TDA) verspricht man sich die Vermeidung von ASD. Da experimentell ermittelte Daten mit der erforderlichen Präzision nicht und klinische Langzeitergebnisse kaum existieren, wird dieses Vorgehen jedoch weiterhin kontrovers diskutiert. Um Rückschlüsse auf unphysiologische Belastung der betroffenen und angrenzenden Segmente ziehen zu können, ist es notwendig, die Segmentkinematik (Änderung von Lage und Ausrichtung der momentanen Rotationsachse) sowie die Steifigkeit und das Bewegungsausmaß (ROM) experimentell zu ermitteln.

Das Ziel dieser Studie war die Analyse des Einflusses der Bandscheibenprothesen Prestige ${ }^{\circledast}$ LP Cervical Disc System (Fa. Medtronic) und Bryan ${ }^{\circledR}$ Cervical Disc System (Fa. Medtronic) auf die Kinematik des Halswirbelsäulen-Segmentes C3/C4.

\subsection{Methodik}

Untersucht wurden die kinematischen Eigenschaften des „Junghanns'schen Segmentes“ C3/C4 von acht menschlichen Halswirbelsäulen. Zunächst erfolgte die genaue Ermittlung und Analyse der physiologischen Eigenschaften des intakten Segmentes. Dazu wurde der C4-Wirbel starr mit der Messapparatur verbunden und ein zyklisches Drehmoment an den jeweiligen C3-Wirbel in axialer $\left(T_{z^{-}}\right)$, sagittaler $\left(T_{x^{-}}\right)$und frontaler Ausrichtung ( $T_{y^{-}}$-Richtung) appliziert. Das experimentell ermittelte Drehmoment betrug 2,25 Nm. Gleichzeitig wurden verschiedene Vorlastgrößen in unterschiedlichen Positionen angelegt. Die resultierende Bewegungsänderung wurde mittels Lagesensoren sechsdimensional, differentiell klein aufgezeichnet. Dabei konnte eine räumliche Auflösung von $<0,01^{\circ}$ für Rotation, sowie $<2,4 \mu \mathrm{m}$ für Translationsbewegungen erreicht werden und die jeweilige Positionsänderung der momentanen Schraubachse („Instantaneous Helical Axis“; IHA) erfasst werden. Im Anschluss an die Bestimmung der physiologischen Charakteristika erfolgte die Resektion der Bandscheibe und deren Ersatz mittels Prestige ${ }^{\circledR}$ LP Cervical Disc System, sowie Bryan ${ }^{\circledR}$ Cervical Disc System. Alle übrigen experimentellen Variablen blieben unverändert. Das Drehmoment wurde bei der Ventralflexion-Extension auf Grund der Reduktion der Steifigkeit und der dadurch enstandenen Stabilität der Segmente auf $1,70 \mathrm{Nm}$ bzw. 0,85 Nm reduziert. Nach der statistischen 
Auswertung und Analyse der kinematischen Eigenschaften beider Bandscheibenprothesen wurden die Ergebnisse mit den Daten der intakten Präparate verglichen und diskutiert.

\subsection{Ergebnisse}

\section{Intaktes Segment}

Das Migrationsverhalten und die Ausrichtung der momentanen Schraubachse zeigten während eines Bewegungszyklus eine ausgeprägte Abhängigkeit von der Richtung des anliegenden Drehmomentes $\left(T_{x} ; T_{y} ; T_{z}\right)$. Bei Ventralflexion-Extension $\left(T_{y}\right)$ verlief die IHA nahezu ortsfest und annähernd parallel zum angreifenden Drehmoment im mittleren Bereich des vierten Halswirbelkörpers. Während der Lateralflexion und axialen Rotation war die Migration der IHA hingegen deutlich stärker ausgeprägt. Im Gegensatz zur Ventralflexion-Extension zeigte sich eine Abhängigkeit des Migrationsverhaltens vom Betrag bzw. Position der axialen Vorlast. Die initiale Schraubachse $\left(\alpha=0^{\circ}\right)$ verlief bei der Lateralflexion und axialen Rotation nicht parallel zur Richtung des entsprechenden Drehmomentes $T_{x}$ bzw. $T_{z}$, sondern von dorso-kranial nach ventro-kaudal in der Sagittalebene, um jeweils $20-30^{\circ}$ zum angreifenden Drehmoment verkippt.

Die Steifigkeit und das Bewegungsausmaß (ROM) des intakten Segmentes zeigten ebenfalls eine Abhängigkeit von der Richtung des applizierten Drehmomentes. Unabhängig vom angreifenden Drehmoment $\left(T_{x} ; T_{y} ; T_{z}\right)$ führte eine Erhöhung der zentralen Vorlast oder eine Verlagerung nach dorsal zu einer Steifigkeitszunahme. Analog dazu kam es zu einer Abnahme des Bewegungsausmaßes. Wurde die Vorlast hingegen nach ventral verschoben, nahm die Steifigkeit bei Axialrotation und Lateralflexion ab, bei Ventralflexion-Extension zu. Gleichzeitig war eine Zunahme des ROM zu verzeichnen. Eine Vorlastverlagerung in seitlicher Richtung zeigte hingegen keinen nennenswerten Effekt auf die Steifigkeit und das Bewegungsausmaß.

\section{$\underline{\text { Prestige }^{\circledR} \text { LP Cervical Disc und Bryan }{ }^{\circledR} \text { Cervical DisC }}$}

Das Migrationsverhalten der IHA wich nach der Implantation der Bandscheibenprothesen sichtbar von den Ergebnissen des intakten Segmentes ab. Dennoch bestand eine gleichbleibende Tendenz des Migrationsverhaltens in Abhängigkeit vom Betrag und der Position der axialen Vorlast, sowie der Richtung des applizierten Drehmomentes $\left(T_{x} ; T_{y} ; T_{z}\right)$. Dabei zeigte sich eine deutliche Verlagerung der IHA nach TDR. Im Vergleich zum intakten Segment kam es bei axialer Rotation unter Prestige ${ }^{\circledR}$ LP (zentrale Vorlast: $20 \mathrm{~N}$ ) zu einem signifikanten Versatz der initialen Schraubachsenpositionen um 5,9 $\mathrm{mm}$ nach dorsal und unter Bryan ${ }^{\circledR}$ Cervical Disc sogar um 8,15 mm. Diese Verschiebung zeigte eine hohe Signifikanz im Vergleich zum intakten Segment mit einem p-Wert von $<0,01$ für Prestige ${ }^{\circledR}$ LP und Bryan ${ }^{\circledast}$. Analog dazu verlagerte sich der Schwerpunkt der gesamten Rastpolkurve (Summe der Durchstoßpunkte der IHA durch eine definierte Ebene) infolge TDR ebenfalls in dorsaler Richtung. 
Gleichzeitig war eine stärkere Abhängigkeit der Lage der momentanen Schraubachse von der sagittalen Vorlastposition zu beobachten.

Die Ausrichtung der momentanen Schraubachse änderte sich in allen drei Bewegungsrichtungen hingegen kaum. Die Neigung der IHA zur Horizontalebene blieb mit $\approx-69^{\circ}$ bei axialer Rotation $\left(T_{z}\right)$ und etwa $26^{\circ}$ bei Lateralflexion $\left(T_{x}\right)$ nahezu konstant, während sie bei Ventralflexion-Extension weiterhin annähernd parallel zum applizierten Drehmoment $\mathrm{T}_{y}$ verlief.

Die Implantation der Bandscheibenprothesen ging mit einer deutlichen Abnahme der Steifigkeit einher. Simultan kam es zu einem Anstieg des Bewegungsausmaßes (ROM). Der Vergleich der relativen Steifigkeit mit dem intakten Segment zeigte bei Prestige ${ }^{\circledR}$ LP annähernd gleichbleibende und von der Vorlastposition unabhängige Steifigkeitswerte. Bei Bryan ${ }^{\circledR}$ Cervical Disc war ein deutlich höherer Effekt auf die Segmentsteifigkeit infolge einer Vorlastverlagerung zu beobachten.

\subsection{Diskussion}

Das individuelle Migrationsverhalten der momentanen Schraubachse wird hauptsächlich durch die Art und Anzahl der in Kontakt tretenden Gelenke (Facetten-, Uncovertebral- und protheseneigene Gelenke) beeinflusst, welche die Bewegung bei Kraftschluss durch die entstandenen Kontaktpunkte führen und die Bewegungsqualität definieren. Nach Implantation der Bandscheibenprothesen zeigt sich eine deutliche Verlagerung der IHA in dorsaler Richtung, was eine Veränderung der Gelenkführung vermuten lässt. Die Kinematik des Indexsegmentes (mit Prothese versorgtes C3/C4Segment) zeigte sich deutlich verändert. Die resultierende Parallelverschiebung der Rotationsachse führt letztendlich zu einer zusätzlichen Translation des oberen Wirbels (C3), was die Kinematik der angrenzenden Segmente beeinflusst.

Die Ausrichtung der IHA bleibt nach TDR für alle Bewegungsrichtungen annähernd konstant. Da sie, wie in anderen Studien der Arbeitsgruppe gezeigt, von der Gelenkführung der Facettengelenke abhängt, kann postuliert werden, dass deren Funktion von der Prothesenimplantation unberührt geblieben ist.

Der deutliche Steifigkeitsverlust nach TDR ist mit der Durchtrennung der Longitudinalbänder einerseits, andererseits mit der Entfernung der Bandscheibe und Teilen der Uncovertebralgelenke zu begründen. Dementsprechend ist ein deutlicher Anstieg des Bewegungsausmaßes unter Prestige ${ }^{\circledR}$ LP und Bryan ${ }^{\circledR}$ Cervical Disc zu beobachten. Dennoch zeigen die Segmentsteifigkeit und das Bewegungsausmaß (ROM) eine deutliche Abhängigkeit von der Position und dem Betrag der axial applizierten Vorlast. Daraus lässt sich schlussfolgern, dass in vivo, sowohl vor, als auch nach Implantation der beiden untersuchten Prothesen, die Eigenschaften des Systems durch unterschiedliche Muskelaktivierungen beeinflusst werden können. 
Die Implantation der untersuchten Bandscheibenprothesen führt zu einer deutlichen Veränderung der kinematischen Eigenschaften des Index-Segmentes. Dabei kommt es zu einer zusätzlichen Translation der beteiligten Wirbel, wodurch auch die Kinematik der benachbarten C2/C3- und C4/C5Segmente beeinflusst wird. Die Entstehung einer Degeneration der angrenzenden Segmente (ALD; „Adjacent Level Disease“) nach totalem Bandscheibenersatz (TDA; „Total Disc Arthroplasty“) ist aus kinematischen Gründen deshalb nicht ausgeschlossen. 
6 Abkürzungsverzeichnis

\begin{tabular}{|c|c|}
\hline Abb. & Abbildung \\
\hline ACDF & Anteriore Zervikale Diskektomie und Fusion \\
\hline AIDS & Acquired Immunodeficiency Syndrome \\
\hline ALD & Adjacent Level Disease \\
\hline ap & anterior-posterior (Strahlengang) \\
\hline Art. & Articulatio \\
\hline Artt. & Articulationes (Mehrzahl) \\
\hline ASD & Adjacent Segment Disease \\
\hline BMI & Body Mass Index \\
\hline BS & Bandscheibe \\
\hline BWS & Brustwirbelsäule \\
\hline bzgl. & bezüglich \\
\hline bzw. & beziehungsweise \\
\hline ca. & circa \\
\hline $\mathrm{cm}$ & Zentimeter \\
\hline COR & Center Of Rotation \\
\hline CT & Computertomographie \\
\hline d.h. & das heißt \\
\hline etc. & et cetera \\
\hline Fa. & Firma \\
\hline FDA & U.S. Food and Drug Administration \\
\hline FEM & Finite Element Method \\
\hline FHA & Finite Helical Axis \\
\hline HAM & Helical Axis of Motion \\
\hline HIV & Humanes Immunodefizienz-Virus \\
\hline HWK & Hals-Wirbel-Körper \\
\hline HWS & Halswirbelsäule \\
\hline IHA & Instantaneous Helical Axis \\
\hline IMR & Initiale Migrations-Rate \\
\hline $\mathrm{I} / \mathrm{min}$ & Liter pro Minute \\
\hline Lig. & Ligamentum \\
\hline Ligg. & Ligamenta (Mehrzahl) \\
\hline LWS & Lendenwirbelsäule \\
\hline M. & Musculus \\
\hline $\mathrm{Mm}$. & Musculi \\
\hline $\mathrm{ml}$ & Milliliter \\
\hline $\mathrm{mm}$ & Millimeter \\
\hline $\mathrm{mm} / \mathrm{deg}$ & Millimeter pro Grad \\
\hline$\mu \mathrm{m}$ & Mikrometer \\
\hline$\mu \mathrm{deg}$ & Mikrograd \\
\hline MRT & Magnetresonanztomographie \\
\hline $\mathrm{N}$ & Newton (Einheit der Kraft) \\
\hline $\mathrm{Ncm}$ & Newtonzentimeter (Einheit der Energie) \\
\hline $\mathrm{Ncm} / \mathrm{deg}$ & Newtonzentimeter pro Grad (Einheit der Steifigkeit) \\
\hline $\mathrm{Nm}$ & Newtonmeter (Einheit der Energie) \\
\hline OPLL & Ossification of the Posterior Longitudinal Ligament \\
\hline Proc. & Processus \\
\hline Procc. & Processus (Mehrzahl) \\
\hline rel & relativ \\
\hline
\end{tabular}




\begin{tabular}{|l|l|}
\hline ROM & Range Of Motion \\
\hline RPK & Rastpolkurve \\
\hline S. & siehe \\
\hline S. & Seite \\
\hline SD $(\sigma)$ & Standardabweichung \\
\hline TDA & Total Disc Arthroplasty \\
\hline TDR & Total Disc Replacement \\
\hline TN & Tennessee \\
\hline UK & United Kingdom \\
\hline VL & Vorlast \\
\hline vgl. & vergleiche \\
\hline WZ & Widerstandszentrum \\
\hline z.B. & zum Beispiel \\
\hline Z.n. & Zustand nach \\
\hline$\Delta x$ & Änderung eines Parameters $x$ \\
\hline$d x$ & Differential eines Parameters $x$ \\
\hline 3D & Drei-dimensional \\
\hline 6D & Sechs-dimensional \\
\hline
\end{tabular}




\section{Literaturverzeichnis}

Ahmed AM, Duncan NA, Burg DL (1990): The effect of facetgeometrie on the axial torque rotation response of the lumbar motion segments. Spine $\underline{15}, 391-401$

Anderson PA, Rouleau JP (2004): Intervertebral disc arthroplasty. Spine 29, 2779-2786

Anderson PA, Sasso RC, Rouleau JP, Carlson CS, Goffin J (2004): The Bryan Cervical Disc: wear properties and early clinical results. Spine J $\underline{4}$ (6 Suppl), 303-309

Aoki Y, Takahashi Y, Takahashi K, Chiba T, Kurokawa M, Ozawa T, Moriya H (2004): Sensory innervation of the lateral portion of the lumbar inervertebral disc in rats. Spine $\underline{4}, \mathbf{2 7 5 - 2 8 0}$

Assmann B, Selke P: Das allgemeine Kräftesystem, In: Statische Mechnik, Band 1: Statik, 18. Auflage, Oldenbourg Wissenschaftsverlag, München 2006, 352-378

Bartels RHMA, Donk RD, Pavlov P, van Limbeek J (2008): Comparison of biomechanical properties of cervical artificial disc prosthesis: A review. Clin Neurol Neurosurg 110, 963-967

Beguiristain JL, Martinez-Peric R, Barrios RH, Villas C (1994): Lumbosacral arthrodesis with louistechnique. Review of 186 cases. Eur Spine J $\underline{3}(3), 169-171$

Bertagnoli R, Yue JJ, Fenk-Mayer A, Eerulkar J, Emerson JW (2006): Treatment of symptomatic adjacent-segment degeneration after lumbar fusion with total disc arthroplasty by using the prodisc prosthesis: a prospective study with 2-year minimum follow up. J Neurosurg Spine $\underline{4}(2), 91-97$

Bockermann V (2004): Kinematik des Halswirbelsäulen-Segments C5/6 - Biomechanische Analyse seines Bewegungsmusters bei physiologischen Standardbelastungen, Med. Diss. Göttingen 2004

Bogduk N, Twomey (1991): Clinical anatomy of the lumbar spine. Churchill Livingstone, London, New York, Tokio 1991

BohImann HH, Emery SE, Goodfellow DB, Jones PK (1993): Robinson anterior cervical discectomy and arthrodesis for cervical radiculopathy. Long-term follow-up of one hundred and twenty-two patients. J Bone Joint Surg Am 75, 1298-1307

Bommas-Ebert U, Teubner P, Voß R: Abbildung 4.1: Linke Seitenansicht der Wirbelsäule. In: Kurzlehrbuch Anatomie und Embryologie, 2. Auflage, Thieme, Stuttgart 2006, 154

Bono CM, Lee CK (2004): Critical analysis of trends in fusion for degenerative disc disease over the past 20 years: influence of technique on fusion rate and clinical outcome. Spine (Phila Pa 1976) 29(4), 455-463

Bryan V (2002) Cervical motion segment replacement. Eur Spine J 11 (Suppl 2), 92-97

Buchowski JM, Anderson PA, Sekhon L, Riew KD (2009): Cervical Disc Arthroplasty Compared with Arthrodesis for theTreatment of Myelopathy: Surgical Technique. J Bone Joint Surg Am 91, 223-232

Chang UK, Kim DH, Lee MC, Willenberg R, Kim SH, Lim J (2007): Changes in adjacent-level disc pressure and facet joint force after cervical arthroplasty compared with cervical discectomy and fusion. J Neurosurg Spine $\underline{7}, 33-39$ 
Cheng BC, Welch WC: Biomechanik von Nonfusion-Implantaten: Neuartige Testverfahren, Standards und Auswirkungen auf zukünftige Implantate. In: Bewegungserhaltende Wirbelsäulenchirurgie, Bertagnoli R, Yue JJ, McAfee PC, An HS (Hrsg.), Urban \& Fischer, München 2011, 30-37

Cherry C (2002): Anterior cervical discectomy and fusion for cervical disc disease. AORN J $\underline{76}$, $998-$ 1008

Chung SK, Kim YE, Wang KC (2009): Biomechanical effect of constraint in lumbar total disc replacement: a study with finite element analysis. Spine (Phila Pa 1976) 34(12), 1281-1286

Cloward, R.B. (1958): The anterior approach for removal of ruptured cervical disks. J Neurosurg $\underline{15}(6)$, 602-617

Cossette JW, Farfan HF, Robertson GH, Wells RV (1971): The instantaneous center of rotation of the third lumbar intervertebral joint. J Biomech $\underline{4}(2), 149-153$

Cripton PA, Bruehlmann SB, Orr TE, Oxland TR, Nolte LP (2000): In vitro axial preload application during spine flexibility testing: towards reduced apparatus-related artefacts. J Biomech $\underline{33}(12), 1559-$ 1568

Cunningham BW, Dmitriev AE, Hu N, McAfee PC (2003): General principles of total disc replacement arthroplasty: seventeen cases in a nonhuman primate model. Spine (Phila Pa 1976) 28 (20), 118-124

Delamarter RB, Pradhan BB: ProDisc-C. In: Bewegungserhaltende Wirbelsäulenchirurgie, Bertagnoli R, Yue JJ, McAfee PC, An HS (Hrsg.), Urban \& Fischer, München 2011, 119-126

Dmitriev AE, Cunningham BW, Hu N, Sell G, Vigna F, McAfee PC (2005): Adjacent level intradiscal pressure and segmental kinematics following a cervical total disc arthroplasty: an in vitro human cadaveric model. Spine (Phila Pa 1976) $\underline{30}(10), 1165-1172$

Drenckhahn D: Allgemeine Gelenk- und Knochenlehre. In: Anatomie, 16. Auflage. Benninghoff A, Drenckhahn D (Hrsg.), Band 1, Urban \& Fischer, München/Jena 2003, 254-277

Eck JC, Humphreys SC, Lim TH, Jeong ST, Kim JG, Hodges SD, An HS (2002): Biomechanical study on the effect of cervical spine fusion on adjacent-level intradiscal pressure and segmental motion. Spine (Phila Pa 1976) 277(22), 2431-2434

Edwards WT, Hayes WC, Posner I, White AA, Mann RW (1987): Varation of lumbar spine stiffnesswith load. J Biomech Eng 109, 35-42

El-Bohy AA, Yang K-H, King Al (1989): Experimental verification of facet load transmission by direct measurements of facet laminar contact pressure. J Biomech 22-8, 931-941

Emery SE, Bohlmann HH, Bolesta MJ, Jones PK (1998): Anterior cervical decompression and arthrodesis for the treatment of cervical spondylotic myelopathy. Two to seventeen-year follow-up. J Bone Joint Surg Am 으, 941-951

Fanghänel J (2001): Existenz von Mechanorezeptoren im Facetten- und Bandapparat der menschlichen Wirbelsäule. Persönliche Mitteilung

Fanghänel J (2009): Ingredients of the preserving solution: Aqua dest, alcohol, glycerine, formalin, thymol, salicylic acid. Personal Communication 
Fanghänel J, Schultz F (1962): Mitteilung über eine Konservierungsflüssigkeit für anatomisches Präpariermaterial. Z Med Labortech $\underline{3}$, 329-332

Farfan HF: Biomechanik der Lendenwirbelsäule ( Die Wirbelsäule in Forschung und Praxis, Bd. 80), Hippokrates-Verlag, Stuttgart 1979

Fennel AJ, Jones AP, Hukins DW (1996): Migration of the Nucleus pulposus within the intervertebral disc during flexion and extension of the spine. Spine 211, 2753-2757

Fercher AF (1999): Elastostatik in Medizinische Physik - Physik für Mediziner, Pharmazeuten und Biologen. 2. Auflage, Springer Wien / New York, 71-89

Fernstrom U (1966) Arthroplasty with intracorporal endoprothesis in herniated disc and in painful disc. Acta Chir Scand Suppl 357, 154-159

Fließbach T: Starre Körper, In: Mechanik - Lehrbuch zur Theoretischen Physik 1, 5. Auflage, Elsevier/Spektrum Akademischer Verlag, München 2007, 165-171

Freeman BJ, Davenport J (2006): Total disc replacement in the lumbar spine: a systematic review of the literature. Eur Spine J 15 Suppl 3, 439-447

Friedrich I, Lühmann J (2005): The cervical spine in training therapy, KCS $2005 \underline{6}$ (3), 61-68

Fritsch EW, Pitzen T (2006): Zervikale Bandscheibenprothesen. Orthopade 35, 347-361

Galbusera F, Bellini CM, Brayda-Bruno M, Fornari M (2008a): Biomechanical studies on cervical total disc arthroplasty: A literature review. Clin Biomech $\underline{23}$, 1095-1104

Galbusera F, Bellini CM, Zweig T, Ferguson S, Raimondi MT, Lamartina C, Brayda-Bruno M, Fornari M (2008b): Design concepts in lumbar total disc arthroplasty. Eur Spine J 17, 1635-1650

Gibson JN, Waddell G (2005): Surgery for degenerative lumbar spondylosis: updated Cochrane

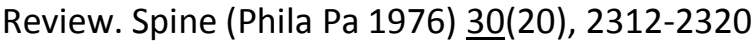

Götz W, Barnert S, Bertagnoli R, Miosge N, Kresse H, Herken R (1997): Immunohistochemical localization of the small proteoglycans decorin and biglycan in human intervertebral discs. Cell Tissue Res $\underline{289}, 185-190$

Götz W, Bertagnoli R, Herken R: Struktur und Zusammensetzung der extrazellulären Matrix normaler menschlicher Nuclei pulposi. In: Die traumatische und degenerative Bandscheibe (Hefte „Der Unfallchirurg“ 271), Wilke HJ, Claes LE (Hrsg.), Springer Verlag, Heidelberg 1999, 3-15

Goffin J, van Loon J, Van Calenbergh F, Plets C (1995): Long-term results after anterior cervical fusion and osteosynthetic stabilization for fractures and/or dislocations of the cervical spine. J Spinal Disord 8(6), 499-508

Goffin J, Casey A, Kehr P, Liebig K, Lind B, Logroscino C, Pointillart V, Van Calenbergh F, van Loon J (2002): Preliminary clinical experience with the Bryan Cervical Disc Prosthesis. Neurosurgery $\underline{51}, 840-$ 847

Goffin J, Geusens E, Vantomme N, Quintens E, Waerzeggers Y, Depreitere B, Van Calenbergh F, van Loon J (2004): Long-term follow-up after interbody fusion of the cervical spine. J Spinal Disord Tech 17, 79-85 
Grifka J, Hedtmann A, Pape HG, Witte H, Bar HF (1998): Beschleunigungsverletzung des HWS. Orthopäde 27, 802-812

Grimmer A, Wurm G (2011): Chirurgische Strategien bei Bandscheibenvorfällen an der Halswirbelsäule. Neurochir Psychiatr 12(1), 23-30

Gumbert N (2012a): HWS-Syndrom Symptome auf URL: http://www.dr-gumpert.de/html/hws_syndrom_symptome.html, Stand 12.12.2012, 15:13 Uhr

Gumbert N (2012b): HWS-Syndrom Ursachen auf URL:

http://www.dr-gumpert.de/html/hws_syndrom_ursachen.html, Stand 12.12.2012, 15:21 Uhr

Habela NM, McAfee PC: Indikationen und Kontraindikationen für die zervikale nicht versteifende Operation: Patientenauswahl. In: Bewegungserhaltende Wirbelsäulenchirurgie, Bertagnoli R, Yue JJ, McAfee PC, An HS (Hrsg.), Urban \& Fischer, München 2011, 56-61

Haberl H, Cripton PA, Orr TE, Beutler T, Frei H, Lanksch WR, Nolte LP (2004): Kinematic response of lumbar functional spinal units to axial torsion with and without superimposed compression and flexion/extension. Eur Spine J 13(6), 560-566

Hartmann M (2012): Proportionen. Auf URL: http://www.zbrush.de/images/uploaded/user_imgvjQ6ipO1LO_proportionen.gif. Stand: 07.02.2012, 17:32 Uhr

Hawellek T (2008): Eine biomechanische Analyse des Lendenwirbelsegments L4/L5. Med. Diss. Göttingen 2008

Hilibrand AS, Robbins M (2004): Adjacent segment degeneration and adjacent segment disease: the consequences of spinal fusion? Spine 4 , 190-194

Hilibrand AS, Carlson GD, Palumbo MA, Jones PK, BohIman HH (1999): Radiculopathy and myelopathy at segments adjacent to the site of a previous anterior cervical arthrodesis. J Bone Joint Surg Am 81(4), 519-528

Jaramillo-de la Torre JJ, Grauer JN, Yue JJ (2008) Update on cervical disc arthroplasty: where are we and where are we going? Curr Rev Musculoskelet Med 1, 124-130

Jensen WK, Anderson PA, Rouleau JP (2005): Bone ingrowth into the Bryan Cervical Disc. Spine $\underline{30}$, 2497-2502

Junghanns H: Nomenclatura columnae vertebralis (Die Wirbelsäule in Forschung und Praxis. Bd. 75), Hippokrates-Verlag, Stuttgart 1977

Kalscheuer A K (2001): Kinematik des Halswirbelsäulensegments C3/C4 unter systematischer Lastvariation. Med. Diss. Göttingen 2001

Kang H, Park P, La Marca F, Hollister SJ, Lin CY (2010a): Analysis of load sharing on uncovertebral and facet joints at the C5-6 level with implantation of the Bryan, Prestige LP, or ProDisc-C cervical disc prosthesis: an in vivo image-based finite element study. Neurosurg Focus $\underline{28}$, E9. doi: 10.3171/2010.3.FOCUS1046

Kang KC, Lee CS, Han JH, Chung SS, (2010b): The factors that influence the postoperative segmental range of motion after cervical artificial disc replacement. Spine $\underline{10}, 689-696$ 
Kapandji IA: Funktionelle Anatomie der Gelenke. Rumpf und Wirbelsäule. Bd. 3, Enke Verlag, Stuttgart 1985

Kettler A, Marin F, Sattelmayer G, Mohr M, Mannel H, Durselen L, Claes L, Wilke HJ (2004): Finite helical axes of motion are a useful tool to describe the three-dimensional in vitro kinematics of the intact, injured and stabilized spine. Eur Spine J 13, 553-559

Kluba T, Roetman B (2005a): Wirbelsäule und Brustkorb. In: Taschenlehrbuch Orthopädie und Unfallchirurgie. Wülker N (Hrsg.), Thieme, Stuttgart 2005, 220-290

Kluba T, Roetman B (2005b): Abbildung 4.7: Schematische Darstellung der Wirbelsäulendegeneration. In: Taschenlehrbuch Orthopädie und Unfallchirurgie. Wülker N (Hrsg.), Thieme, Stuttgart 2005, 235

Kluba T, Roetman B (2005c): Abbildung 4.9: Verschiedene Schweregrade der Bandscheibendegeneration. In: Taschenlehrbuch Orthopädie und Unfallchirurgie. Wülker N (Hrsg.), Thieme, Stuttgart 2005, 236

Köster G (1985): Das Rezidiv des Bandscheibenvorfalls. Untersuchungen zur Morphologie und Ätiologie im Hinblick auf die operative Therapie. Med. Diss. Göttingen 1985

Koller M (2011): Therapie bei Bandscheibenerkrankungen. Journal für Neurologie. Neurochir Psychiatr $\underline{12}(1), 16-21$

Kotani Y, Cunningham BW, Abumi K, Dmitriev AE, Ito M, Hu N, Shikinami Y, McAfee PC, Minami A (2005): Multidirectional flexibility analysis of cervical artificial disc reconstruction: in vitro human cadaveric spine model, J Neurosurg Spine 2, 188-194

Kowalczyk I, Lazaro BC, Fink M, Rabin D, Duggal N (2011): Analysis of in vivo kinematics of 3 different cervical devices: Bryan disc, ProDisc-C, and Prestige LP disc. J Neurosurg Spine $\underline{15}$, 630-635

Krämer J: Bandscheibenbedingte Erkrankungen. 2. Auflage, Thieme, Stuttgurt 1987

Krag MH, Seroussi RE, Wilder DG, Pope MH (1987): Internal displacement distribution from in vitro loading. Spine 12, 1001-1007

Krismer M: Die Rotation der Brust- und Lendenwirbelsäule; Aktuelle Probleme in Chirurgie und Orthopädie. Bd. 43; Verlag Hans Huber, Bern/Göttingen/Toronto/Seattle 1996

Kubein-Meesenburg D, Nägerl H, Cotta H, Fanghänel J (1993): Biomechanische Prinzipien in Diarthrosen und Synarthrosen. Teil I: Grundbegriffe bei Diarthrosen. Z Orthop 131, 97-104

Lazaro BC, Yucesoy K, Yukel KZ, Kowalczyk I, Rabin D, Fink M, Duggal N (2010): Effect of arthroplasty design on cervical spine kinematics: analysis of the Bryan Disc, ProDisc-C, and Synergy disc. Neurosurg Focus $\underline{28}$, E6

Leidel BA, Kirchhoff C, Keßler S, Mutschler W (2008): Trauma der Halswirbelsäule. Gutachterliche Herausforderungen nach Beschleunigungverletzung der HWS. Orthopade $\underline{37}, 414-423$

Lindahl O (1976): Mechanical properties of dried defatted spongy bone. Acta Orthop Scand 47, 11-19

Lippert: Rückenmark. In: Lehrbuch Anatomie, 8. Auflage, Urban \& Fischer, München 2011, 143-154 
Lu Y, Chen C, Kallakuri S, Patwardhan A, Cavanaugh JM (2005): Development of an in vivo method to investigate biomechanical and neurophysiological properties of spine facet joint capsules. Eur Spine $J$ $\underline{14}(6), 565-572$

Luschka H: Die Nerven des menschlichen Wirbelkanals. H Laub, Tübingen 1856

Lutz G (1967): Die Entwicklung der kleinen Wirbelgelenke. Z Orthop 104, 19-28

Mansour M (2001): Die biomechanische Funktion der Artikulationes zygapophysiales der Lendenwirbelsäule. Med. Diss. Göttingen 2001

Mansour M, Spiering S, Lee C, Dathe H, Kalscheuer AK, Kubein-Meesenburg D, Nägerl H (2004): Evidence for IHA migration during axial rotation of a lumbar spine segment by using a novel highresolution $6 \mathrm{D}$ kinematic tracking system. Journal of biomechanics $\underline{37}, 583-592$

McAfee PC, Cunningham BW, Orbegoso CM, Sefter JC, Dmitriev AE, Fedder IL (2003): Cervical disc replacement with porous coated motion prosthesis: a comparative biomechanical analysis showing the key role of the posterior longitudinal ligament. Spine $\underline{28}$ (Suppl), 176-185

McAfee PC, Cunningham BW, Hayes V, Sidiqi F, Dabbah M, Sefter JC, Hu N, Beatson H (2006):

Biomechanical analysis of rotational motions after disc arthroplasty: implications for patients with adult deformities. Spine $\underline{31}, 152-160$

McKenzie AH (1972): Steel ball arthroplasty of lumbal discs. J Bone Joint Surg Br $\underline{54}, 266$

McKenzie AH: Die Grundlagen der bewegungserhaltenden Chirurgie: Lektionen aus der Vergangenheit. In: Bewegungserhaltende Wirbelsäulenchirurgie, Bertagnoli R, Yue JJ, McAfee PC, An HS (Hrsg.), Urban \& Fischer, München 2011, 2-10

Medtronic (2012):-Medtronic Launches BRYAN ${ }^{\circledR}$ Cervical Disc with ACD Instrument Set. Auf URL: http://wwwp.medtronic.com/Newsroom/NewsReleaseDetails.do?itemld=1349450388104\&lang=en_ US, Stand: 03.01.2013 19:33 Uhr

Medtronic (2013): About the PRESTIGE ${ }^{\circledR}$ LP Cervical Disc. Auf URL:

http://www.prestigedisc.com/intl/about-prestige-disc.html, Stand: 03.01.2013 19:33 Uhr

Medtronic Sofamor Danek (2004): Prestige ${ }^{\circledR}$ LP Cervical Disc System Surgical Technique

Mehren C, Mayer HM (2005): Artificial cervical disc replacement - An update. Neurol India $\underline{53}, 440-$ 444

Mercer S, Bogduk N (1999): The ligaments and annulus fibrosus of human adult cervical intervertebral discs. Spine 24, 619-626

Meyer E, Guicking D: Schwingungslehre. F.Vieweg, Braunschweig 1974

Mummaneni PV, Haid RW (2004): The future in the care of the cervical spine: interbody fusion and arthroplasty. J Neurosurg (Spine 1), 2, 155-159

Mummaneni PV, Robinson JC, Haid RW (2007): Cervical arthroplasty with the Prestige LP Cervical Disc. J Neurosurg, 60, 310-315 
Nägerl H (1990): Biomechanische Prinzipien in Diarthrosen und Synarthrosen. Med. Habil.- Schr. Göttingen 1990

Nägerl H, Kubein-Meesenburg D, Fanghänel J (1992): Elements of a general theory of joints. 7. Mechanical structures of the relative motion of adjacent vertebrae. Ann Anat 174, 66-75

Nägerl H, Kubein-Meesenburg D, Cotta H, Fanghänel J, Rossow A, Spiering S (1995): Biomechanical principles in diarthroses and synarthroses. IV: the mechanics of lumbar vertebrae. A pilot study. Z Orthop ihre Grenzgeb $\underline{133}$, 481-491

Nägerl H, Hawellek T, Lehmann A, Hubert J, Saptschak J, Dörner J, Raab BW, Fanghänel J, KubeinMeesenburg D, Wachowski MM (2009): Non-linearity of flexion-extension characteristics in spinal segments. Acta Bioeng Biomech $\underline{11}$ (4), 3-8

Niethard FU, Pfeil J: Degenerative Wirbelsäulenerkrankungen. In: Duale Reihe Orthopädie, 5.Auflage, Thieme Stuttgart 2005, 363-375

Niosi CA, Zhu QA, Wilson DC, Keynan O, Wilson DR, Oxland TR (2006): Biomechanical characterization of the three-dimensional kinematic behaviour of the Dynesys dynamic stabilization system: an in vitro study. Eur Spine J 15(6), 913-922

Ohtori S, Takaschi K, Chiba T, Yamagata M, Sameda H, Moriya H (2001): Sensory innervation of the cervical facet joints in rats. Spine $\underline{26}, 147-150$

Oxland TR, Panjabi MM, Lin RM (1994): Axes of motion of thoracolumbar burst fractures. J Spinal Disord $\underline{7}(2), 130-138$

Panjabi MM, Brand RM, White AA (1976): Three dimensional flexibility and stiffness of human thoracic spine. J Biomech $\underline{9}, 185-192$

Panjabi MM, Oxland TR, Yamamoto I, Crisco JJ (1994): Mechanical behavior of the human lumbar and lumbosacral spine as shown by three-dimensional load-displacement curves. J Bone Joint Surg Am 76(3), 413-424

Parnianpour M, Li F, Nordin M, Kahanovitz N (1989): A data base of isointerial trunk strength test against three resistance level in sagittal frontal and transverse plancs in normal male subjects. Spine $\underline{14}, 409-411$

Patwardhan AG, Tzermiadianos MN, Tsitsopoulos PP, Voronov LI, Renner SM, Reo ML, Carandang G, Ritter-Lang K, Havey RM (2010): Primary and coupled motions after cervical total disc replacement using a compressible six-degree-of-freedom prosthesis. Eur Spine J, 21 (Suppl 5), 618-629

Phillips FM, Garfin SR (2005): Cervical disc replacement, Spine $\underline{30}$ (17 Suppl), 27-33

Plato (1950): Dialogues of Plato. U.S.A., Pocket Books, Inc.

Platzer W (1999a): Allgemeine Anatomie. In: Taschenatlas Anatomie in 3 Bänden, 7. Auflage, Band 1 Bewegungsapparat, Thieme, Stuttgart 1999, 2-33

Platzer W (1999b): Stamm. In: Taschenatlas Anatomie in 3 Bänden, 7. Auflage, Band 1

Bewegungsapparat, Thieme, Stuttgart 1999, 36-107 
Platzer W (1999c): Taschenatlas Anatomie in 3 Bänden, 7. Auflage, Band 1 Bewegungsapparat, Thieme, Stuttgart 1999

Poeck H, Hacke W: Erkrankungen der Bandscheibe. In: Neurolgie, 12. Auflage, Springer, Heidelberg 2006, 647-658

Puttlitz CM, Rousseau MA, Xu Z, Hu S, Tay BK, Lotz JC (2004): Intervertebral disc replacement maintains cervical spine kinetics. Spine (Phila Pa 1976) 29(24), 2809-2814

Putz R: Funktionelle Anatomie der Wirbelsäule (Normale und pathologische Anatomie), Band 43, Thieme, Stuttgart 1981

Putz R: Wirbelsäule, Columna vertebralis. In: Makroskopische Anatomie, Embryologie und Histologie des Menschen. Drenckhan D, Zenker W (Hrsg.), Band 1, Urban \& Schwarzenberg, München 2003

Putz R, Müller-Gerbl M: Rumpf. In: Anatomie, 16. Auflage. Benninghoff A, Drenckhahn D (Hrsg.), Band 1, Urban \& Fischer, München/Jena 2003, 412 - 481

Putz R, Pabst R: Abbildung 793: Zervikales Bewegungssegment; Schema, Medianschnitt. In: Sobotta Atlas der Anatomie des Menschen, 22.Auflage. Band 2, Urban \& Fischer, München 2004, 25

Reichmann S (1971): The postnatal development of form and orientation of the lumbar intervertebral joint surfaces. Z Anat Entwickl-Gesch 133, 102-123

Reinhold M, Blauth M, Rosiek R, Knop C (2006): Verletzungen der unteren Halswirbelsäule. Unfallchirurg $\underline{109}$, 471-482

Reitz H, Joubert MJ (1964): Intractable headache and cervico-brachialgia treated by complete replacement of cervical intravertebral discs with a metal prosthesis. S Afr Med J $\underline{38}, 881-884$

Rieke CC, "Symptoms of imbalance associated with cervical spine pathology" (2008). Independent Studies and Capstones. Paper 293. Program in Audiology and Communication Sciences, Washington University School of Medicine.

Roaf R (1960): A study of the mechanics of spine injuries. J Bone Joint Surg $\underline{42 B}, 810-823$

Rössler H, Rüther W: Krankheiten der Wirbelsäule. In: Orthopädie und Unfallchirurgie, 19.Auflage, Urban \& Fischer, München 2005, 354-394

Rohlmann A, Mann A, Zander T, Bergmann G (2009): Effect of an artificial disc on lumbar spine biomechanics: a probabilistic finite element study. Eur Spine J $\underline{18}(1), 89-97$

Rousseau MA, Bradford DS, Hadi TM, Pedersen KL, Lotz JC (2006): The instant axis of rotation influences facet forces at L5/S1 during flexion/extension and lateral bending. Eur Spine J 15(3), 299307

Sasso R, Schmucker JD, Hacker RJ, Heller JG (2007): Artificial Disc Versus Fusion: A prospective, randomized study with 2-year follow-up on 99 patients. Spine 32(26): 2933-2942

Sasso R, Martin jr. L (2011a): Bryan-Disc. In: Bewegungserhaltende Wirbelsäulenchirurgie, Bertagnoli R, Yue JJ, McAfee PC, An HS (Hrsg.), Urban \& Fischer, München 2011, 111-116 
Sasso R, Martin jr. L (2011b): Bryan-Disc - Abb. 3.9. In: Bewegungserhaltende Wirbelsäulenchirurgie, Bertagnoli R, Yue JJ, McAfee PC, An HS (Hrsg.), Urban \& Fischer, München 2011, 113

Schadé JP: Abbildung Wirbelsäule. In: Lexikon Medizin und Gesundheit, Serges Medien GmbH, Köln 2001,711

Schäfer W (1995): Messung der räumlichen Mikrobewegung und der Verbiegung des Femurschaftes von Hüft-Endo-Prothesen in Abhängigkeit eines räumlichen Kraftsystems. Phys. Dipl. Göttingen 1995

Schildhauer T, Robie BH, Klein R, O'Leary P (1994): The effect of joint compressive load on the motion of the human lumbo-sacral spine. In: Proceedings of the $40^{\text {th }}$. Orthopaedic Research Society, o.O.

Schmidt, H., Heuer, F., Claes, L., \& Wilke, H.J. (2008a). The relation between the instantaneous center of rotation and facet joint forces - A finite element analysis. Clin Biomech $\underline{23}(3), 270-278$

Schmidt, H., Heuer, F., \& Wilke, H.J. (2008b). Interaction between finite helical axes and facet joint forces under combined loading. Spine $\underline{33}(25), 2741-2748$

Schmidt $H$, Heuer F, Wilke HJ (2009): Which axial and bending stiffnesses of posterior implants are required to design a flexible lumbar stabilization system? J Biomech $\underline{42}, 48-54$

Schmitt KU, Walz F, Vetter D, Muser M (2003): Whiplash injury: cases with a long period of sick leave need biomechanical assessment. Eur Spine J 12, 247-254

Sekhon LH.S, Ball JR (2005): Artificial cervical disc replacement: Principles, types and techniques. Neurol India $\underline{53}, 445-50$

Seo M, Choi D (2008): Adjacent segment disease after fusion for cervical spondylosis; myth or reality? Br J Neurosurg 22 (2), 195-199

Sethi RK, Metz LN, Bradford DS: Geschichte und Evolution der Bewegungserhaltung. In:

Bewegungserhaltende Wirbelsäulenchirurgie, Bertagnoli R, Yue JJ, McAfee PC, An HS (Hrsg.), Urban \& Fischer, München 2011, 10-20

Smith GW, Robinson RA (1958): The treatment of certain cervical-spine disorders by anterior removal of the intervertebral disc and interbody fusion. J Bone Joint Surg Am 40A, 607-624

Snyder GM, Bernhardt M (1989): Anterior cervical fractional interspace decompression for treatment of cervical radiculopathy. A review of the first 66 cases. Clin Orthop $\underline{246}, 92-99$

Snyder JT, Tzermiadianos MN, Ghanayem AJ, Voronov LI, Rinella A, Dooris A, Carandang G, Renner SM, Havey RM, Patwardhan AG (2007): Effect of uncovertebral joint excision on the motion response of the cervical spine after total disc replacement. Spine 32, 2965-2969

Spiering S (1995): Kinematik der Lendenwirbelsäule unter Lastvariationen. Phys. Dipl. Göttingen 1995

Tanaka N, Fujimotot Y, An HS, Ikuta Y, YasudaM (2000): The Anatomic Relation Among the Nerve Roots, Intervertebral Foramina and Intervertebral Disks of the Cervical Spine. Spine 25, 286-291

Teichmann H: Physikalische Anwendungen der Vektor- und Tensorrechnung. Band 1, BI-Verlag, Mannheim/Wien/Zürich 1973

Tillmann BN: Atlas der Anatomie des Menschen. Springer, Berlin-Heidelberg 2005 
Thron A: Abb. Bandscheibenvorfall zwischen HWk 4 und 4 im MRT (T1). In: Neurologie, 12. Auflage, Poeck H, Hacke W (Hrsg.), Springer, Heidelberg 2006, 650

Töndury G (1955): Zur Anatomie und Entwicklungsgeschichte der Wirbelsäule mit besonderer Berücksichtigung der Altersveränderungen des Bandscheiben. Schweiz Med Wochenschr $\underline{85}$, 825-837

Töndury G, Theiler K: Entwicklungsgeschichte und Fehlbildungen der Wirbelsäule. HippokratesVerlag, Stuttgart 1990

Tonetti J, Potton L, Riboud R, Peoc'h M, Passagia JG, Chirossel JP (2005): Morphological cervical disc analysis applied to traumatic and degenerative lesions. Surg Radiol Anat 57(4), 693-698

Traynelis VC: Prestige ST und LP. In: Bewegungserhaltende Wirbelsäulenchirurgie, Bertagnoli R, Yue JJ, McAfee PC, An HS (Hrsg.), Urban \& Fischer, München 2011, 117-119

U.S. Food and Drug Administration 2009: PRESTIGE ${ }^{\circledR}$ Cervical Disc System - P0600018 auf URL: http://www.fda.gov/MedicalDevices/ProductsandMedicalProcedures/DeviceApprovalsandClearance s/Recently-ApprovedDevices/ucm076928.htm, Stand: 05.01.2013 12:04 Uhr

U.S. Food and Drug Administration 2012: Bryan ${ }^{\circledR}$ Cervical Disc - P060023 auf URL: http://www.fda.gov/MedicalDevices/ProductsandMedicalProcedures/DeviceApprovalsandClearance s/Recently-ApprovedDevices/ucm162968.htm, Stand: 05.01.2013 14:21 Uhr

Vanselow H: Abbildung C-1.11: Funktion der Disci intervertebrales (Druck- und Zugkräfte). In: Duale Reihe Anatomie, Bob A und K (Hrsg.), Thieme, Stuttgart 2007, 236

Villas C, Martinez-Peric R, Preite R, Barrios RH (1994): Union after multiple anterior cervical fusion. 21 cases followed for 1-6 years. Acta Orthop Scand $\underline{65}(6), 620-622$

Wachowski MM (2005): Biomechanik des C3/C4-Segments; Bedeutung der Unkovertebral- und Wirbelbogengelenke für die Kinematik und Steifigkeit. Med. Diss. Göttingen, 2005

Wachowski MM, Ackenhausen A, Dumont C, Fanghänel J, Kubein-Meesenburg D, Nägerl H (2007): Mechanical properties of cervical motion segments. The archive of mechanical engineering LIV (1), 515

Wachowski MM, Mansour M, Lee C, Ackenhausen A, Spiering S, Fanghänel J, Dumont C, KubeinMeesenburg D, Nägerl H (2009a): How do spinal segments move? J Biomech 42, 2286-2293

Wachowski MM, Hubert J, Hawellek T, Mansour M, Dörner J, Kubein-Meesenburg D, Fanghänel J, Raab BW, Dumont C, Nägerl H (2009b): Axial rotation in the lumbar spine following axial force wrench. J Physiol Pharmacol $\underline{60}$ (Suppl 8), 61-64

Wachowski MM, Mansour M, Hawellek T, Kubein-Meesenburg D, Hubert J, Nägerl H (2009c): Parametric Control of the Stiffness of Lumbar Segments. Strain $2010 \underline{47(3), 281-287}$

Wachowski MM, Hawellek T, Hubert J, Lehmann A, Mansour M, Dumont C, Dörner J, Raab BW, Kubein-Meesenburg D, Nägerl H (2010): Migration of the instantaneous axis of motion during axial rotation in lumbar segments and role of the zygapophysial joints. Acta Bioeng Biomech 12, 39-47

Wachowski MM, Wagner M, Weiland J, Dörner J, Raab BW, Dathe H, Gezzi R, Kubein-Meesenburg D, Nägerl H (2013): Does total disc arthroplasty in C3/C4-segments change the kinematic features of axial rotation?; 10.1016/j.jbiomech.2013.03.027 
Weidl T (2003): Der Schwerpunkt einer Kurve. Auf URL: http://www.iadm.unistuttgart.de/LstAnaMPhy/Weidl/analysis/vorlesung-analysis/node244.html, Stand: 09.01.2013, 22:26 Uhr

Wenig CM, Schmidt CO, Kohlmann T, Schweikert B (2008): Costs of back pain Germany. Eur J Pain $\underline{13}$, 280-286

White AA, Panjabi MM (1978): The basic kinematics of the human spine. A review of past and current knowledge. Spine $\underline{3}(1), 12-20$

White AA, Panjabi MM (1990): Clinical biomechanics of the spine, Second Edition. Lippincott Philadelphia

Wigfield C, Gill S, Nelson R, Langdon I, Metcalf N, Robertson J (2002): Influence of an artificial cervical joint compared with fusion on adjacent-level motion in the treatment of degenerative cervical disc disease. J Neurosurg, $\underline{96}, 17-21$

Wilke HJ, Krischak S, Claes LE, (1996): Formalin fixation strongly influences biomechanical properties of the spine. J Biomech 29 (12), 1629-1631

Wolf K: Lehrbuch der Technischen Mechanik starrer Systeme. Springer, Wien 1931

Wolf K: Lehrbuch der technischen Mechanik starrer Systeme. Springer, Wien 1947

Wurzinger LJ: Rückenmuskulatur. In: Duale Reihe Anatomie, Bob A und K (Hrsg.), Thieme, Stuttgart 2007, 248-255

Zander T, Rohlmann A, Bergmann G (2009): Influence of different artificial disc kinematics on spine biomechanics. Clin Biomech, 24(2), 135-142

Zhu Q, Larson CR, Sjovold SG, Rosler DM, Keynan O, Wilson DR, Cripton PA, Oxland TR (2007):

Biomechanical evaluation of the Total Facet Arthroplasty System: 3-dimensional kinematics. Spine $\underline{32}(1), 55-62$ 


\section{Danksagung}

Die vorliegende Dissertationsarbeit ist in der interdisziplinären Arbeitsgruppe Biomechanik Göttingen-Greifswald entstanden. Beteiligt waren die Abteilung Unfallchirurgie, Plastische und Wiederherstellungschirurgie (Prof. Dr. K. M. Stürmer), die Abteilung Kieferorthopädie (Prof. Dr. D. Kubein-Meesenburg) im Zentrum Zahn-, Mund- und Kieferheilkunde der Georg-August-Universität Göttingen und das Anatomische Institut (Prof. Dr. J. Fanghänel) der Ernst-Moritz-Arndt-Universität Greifswald. Zahlreiche Menschen waren an der Entstehung dieser Arbeit beteiligt, denen ich hiermit meinen herzlichen Dank aussprechen möchte. Ohne ihre tatkräftige Unterstützung wäre diese Arbeit nicht realisierbar gewesen.

Mein außerordentlicher Dank gilt PD Dr. M. Wachowski. Ohne seine vielseitige Unterstützung und die hervorragende Zusammenarbeit wäre diese Arbeit nie zustande gekommen. Ich danke ihm für die Einweisung im Umgang mit der Messapparatur, seine erstklassige wissenschaftliche Anleitung und die unermüdliche und motivierende Diskussions- und Hilfsbereitschaft.

Ebenfalls danke ich Herrn Prof. Dr. H. Nägerl für seine ständige und freundliche Unterstützung, für die anregenden Diskussionen und die hilfreiche, konstruktive Kritik.

Ich danke Herrn Prof. Dr. D. Kubein-Meesenburg für die Möglichkeit, die Räumlichkeiten, sowie die technische Ausstattung der Abteilung Kieferorthopädie nutzen zu dürfen, sowie für das stetige Interesse an der Arbeit.

Mein besonderer Dank gilt Herrn Dr. R. Gezzi und Herrn Dr. H. Dathe für die umfangreiche Unterstützung in der statistischen Verarbeitung und Auswertung der ermittelten Daten. Ihre fachliche Beratung und Mitarbeit trugen entscheidend zur Qualität dieser Arbeit bei.

Herrn Prof. Dr. J. Fanghänel gilt mein Dank für die Bereitstellung, sowie die Anleitung im Umgang mit den anatomischen Präparaten.

Ich danke Herrn Dr. Dörner für die Anleitung und Unterstützung bei der fachgerechten Implantation der untersuchten Bandscheibenprothesen und Herrn Dr. Ortwien-Schunk für die Bereitstellung der dafür nötigen Operationsräumlichkeiten und Instrumente.

Mein Dank gilt ebenfalls der Firma Medtronic Sofamor Danek für die Bereitstellung der untersuchten Bandscheibenprothesen und der zur fachgerechten Implantation nötigen Instrumentarien.

Herrn Dr. S. Spiering danke ich für die Hilfe bei der Weiterentwicklung der Software der Messapparatur und der somit verbesserten Datenverarbeitung.

Des Weiteren bedanke ich mich bei der radiologischen Abteilung der Georg-August-Universität Göttingen für die Untersuchung und Begutachtung der anatomischen Präparate.

Ich danke Frau A. Lehmann und Herrn J. Weiland für die gute Zusammenarbeit und gegenseitige Rücksichtnahme innerhalb der Arbeitsgruppe, sowie für die rege Diskussionsbereitschaft. 
Abschließend möchte ich mich herzlich bei meiner Familie, meinen Freunden und Frau K. Mönninghoff für die ständige Ermunterung und anhaltende Geduld bedanken, mit welcher sie die Entstehung der Arbeit verfolgt und unterstützt haben.

In stillem Andenken danke ich all den Menschen, die sich dazu entschlossen haben, ihren Körper der Wissenschaft zur Verfügung zu stellen. Ihnen gilt meine tiefste Anerkennung. 


\section{Lebenslauf}

Am 11.05. 1987 wurde ich als erster Sohn des Ehepaares Waltraud und Bernhard Wagner in der Lutherstadt Wittenberg geboren. Ich wuchs in dem sächsischen Ort Mockrehna auf und besuchte dort von 1993 bis 1997 die Grundschule Mockrehna. Anschließend setzte ich von 1997 bis 2005 meine schulische Ausbildung am Karl-August-Möbius-Gymnasium in Eilenburg fort und schloss diese im Juli 2005 mit dem Abitur ab. Bis zur Aufnahme meines Studiums absolvierte ich diverse Praktika, unter anderem im Betreuungsverein in Peine, der Fernwasserversorgung Elbaue-Ostharz und im Jugendhaus St. Michael in Roßbach. Im April 2006 nahm ich schließlich das Studium der Humanmedizin an der Georg-August-Universität in Göttingen auf. Im April 2008 absolvierte ich den ersten Abschnitt der ärztlichen Prüfung (Physikum). Während des klinischen Abschnittes meines Medizinstudiums famulierte ich in der Akutpsychiatrie im Asklepios Fachklinikum in Göttingen, sowie im Fachgebiet Innere Medizin im Kreiskrankenhaus Torgau und der Gemeinschaftspraxis Dr. Baumgärtel/Dr. Gerke/Dr. Weiß in Duderstadt. Zudem bestand meinerseits früh ein besonderes Interesse für das Fachgebiet Augenheilkunde, sodass ich in der Gemeinschaftspraxis Dr. Henjes/Hessel/Melchert in Torgau, dem Kreiskrankenhaus Rudolf Virchow in Glauchau und der Augenuniversitätsklinik in Leipzig praktische Erfahrungen im Rahmen diverser Famulaturen sammelte. Im Rahmen des Praktischen Jahres war ich zunächst in der Unfallchirurgie des Evangelischen Krankenhauses Oldenburg und in der Allgemeinchirurgie des Pius Hospitals Oldenburg tätig. Im Klinikum Oldenburg absolvierte ich das zweite Tertial im Fachgebiet Innere Medizin und das drittel Tertial im Fach Augenheilkunde im Universitätsklinikum Göttingen. Des Weiteren war ich während meines Studiums als Hilfswissenschaftler im anatomischen Präparierkurs, sowie in der Abteilung Augenheilkunde tätig und arbeitete darüber hinaus als studentischer Tutor für die Fächer Augenheilkunde und Neurologie im Studentischen Trainingszentrum ärztlicher Praxis und Simulation (STÄPS) in Göttingen. Im November 2012 schloss ich das Studium der Humanmedizin erfolgreich mit dem medizinischen Staatsexamen (zweiter Abschnitt der ärztlichen Prüfung) ab. Anschließend widmete ich mich als Promotionsstudent ausschließlich meiner Promotionsarbeit, bis ich am 01. April 2013 meine ärztliche Tätigkeit in der Augenklinik der Otto-von-Guericke-Universität in Magdeburg aufnahm. 